\title{
Myocellular lipid droplets and insulin sensitivity
}

Citation for published version (APA):

Gemmink, A. (2017). Myocellular lipid droplets and insulin sensitivity: a microscopy approach. [Doctoral Thesis, Maastricht University]. Maastricht University. https://doi.org/10.26481/dis.20170524ag

Document status and date:

Published: 01/01/2017

DOI:

10.26481/dis.20170524ag

Document Version:

Publisher's PDF, also known as Version of record

Document license:

Unspecified

\section{Please check the document version of this publication:}

- A submitted manuscript is the version of the article upon submission and before peer-review. There can be important differences between the submitted version and the official published version of record.

People interested in the research are advised to contact the author for the final version of the publication, or visit the DOI to the publisher's website.

- The final author version and the galley proof are versions of the publication after peer review.

- The final published version features the final layout of the paper including the volume, issue and page numbers.

Link to publication

\footnotetext{
General rights rights.

- You may freely distribute the URL identifying the publication in the public portal. please follow below link for the End User Agreement:

www.umlib.nl/taverne-license

Take down policy

If you believe that this document breaches copyright please contact us at:

repository@maastrichtuniversity.nl

providing details and we will investigate your claim.
}

Copyright and moral rights for the publications made accessible in the public portal are retained by the authors and/or other copyright owners and it is a condition of accessing publications that users recognise and abide by the legal requirements associated with these

- Users may download and print one copy of any publication from the public portal for the purpose of private study or research.

- You may not further distribute the material or use it for any profit-making activity or commercial gain

If the publication is distributed under the terms of Article $25 \mathrm{fa}$ of the Dutch Copyright Act, indicated by the "Taverne" license above, 
Myocellular lipid droplets and insulin sensitivity:

a microscopy approach 
This thesis is part of NanoNextNL, a micro and nanotechnology innovation consortium of the Government of the Netherlands and 130 partners from academia and industry. More information on www.nanonextnl.nl. The research presented in this thesis was performed within NUTRIM School of Nutrition and Translational Research in Metabolism.
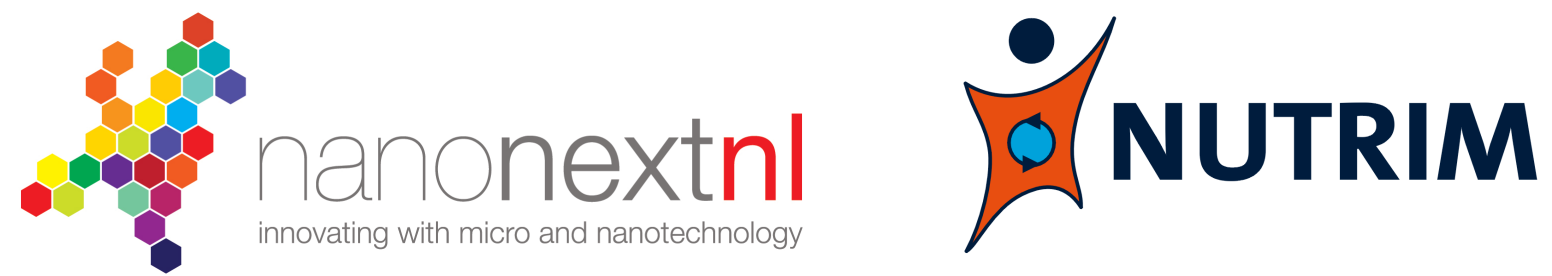

\section{Copyright @ 2017 Anne Gemmink, Maastricht}

Cover design: Anne Gemmink

Lay Out: Anne Gemmink

Printed by: Gildeprint - Enschede

ISBN: 978-94-6233-615-5 


\section{Myocellular lipid droplets and insulin sensitivity: a microscopy approach}

\section{PROEFSCHRIFT}

ter verkrijging van de graad van doctor aan de Universiteit Maastricht, op gezag van de Rector Magnificus, Prof dr. Rianne M. Letschert volgens het besluit van het College van Decanen, in het openbaar te verdedigen op woensdag 24 mei 2017 om 14.00 uur

door

Anne Gemmink 
Promotores

Prof. dr. M.K.C. Hesselink

Prof. dr. P. Schrauwen

\section{Copromotor}

Prof. dr. M.A.M.J. van Zandvoort (RWTH Aachen, Maastricht University)

\section{Beoordelingscommissie}

Prof. dr. R. Mensink (voorzitter)

Prof. dr. B.H. Goodpaster (Translational Research Institute for Metabolism and Diabetes,

Orlando, Fl, USA)

Prof. dr. R.M.A. Heeren

Dr. C. Moro (University of Toulouse, France)

Prof. dr. F.C.S. Ramaekers 


\section{TABLE OF CONTENTS}

$\begin{array}{lll}\text { Chapter } 1 & \text { General Introduction } & 7\end{array}$

Chapter 2 Lipid droplet dynamics and insulin sensitivity upon a 5-day high-fat diet in Caucasians and South Asians

Chapter 3 A microscopy journey to examine lipid droplets, Perilipin 5, and mitochondria in muscle

Chapter 4 Decoration of intramyocellular lipid droplets with PLIN5 modulates fasting-induced insulin resistance and lipotoxicity in humans

Chapter 5 Dissociation of intramyocellular lipid storage and insulin resistance in trained athletes and patients with type 2 diabetes; involvement of the lipid droplet coat protein Perilipin 5

Chapter 6 Different intramyocellular lipid storage pattern and level of lipid droplet saturation in athletes and type 2 diabetes patients

Chapter 7 A microscopic view of intracellular cardiac lipid storage in humans

Chapter 8 General Discussion

Appendices Summary

Samenvatting

Valorization

Risk analysis and technology assessment

Dankwoord

List of publications

Curriculum Vitae 



\section{CHAPTER 1}

\section{General Introduction}

Adapted from Gemmink, Anne, Hesselink, Matthijs. Intramyocellular lipid storage and insulin resistance [internet]. 2015 Feb 11; Diapedia 3105512811

rev. no. 7 
The prevalence of type 2 diabetes is increasing worldwide [1]. Type 2 diabetes coincides with insulin resistance of the skeletal muscle. Insulin resistance is associated with high levels of intramyocellular lipid (IMCL), which is predominantly dispersed into small lipid droplets (LDs) throughout the muscle fibers (Figure 1). In insulin resistant white adipose tissue, insulin mediated suppression of lipolysis is compromised; hence circulating levels of fatty acids are high [2]. Sequestering of fatty acids from the circulation in non-adipose tissue (like skeletal muscle) is referred to as ectopic lipid storage. The capacity to oxidize fatty acids is compromised in most insulin resistant subjects [3]. Thus, spillover of fatty acids from the white adipose tissue along with a reduced fat oxidative capacity may promote lipid storage in muscle. Multiple studies have reported a negative correlation between IMCL content and insulin sensitivity [4-6], suggesting that a high IMCL content in skeletal muscle is detrimental for insulin sensitivity.

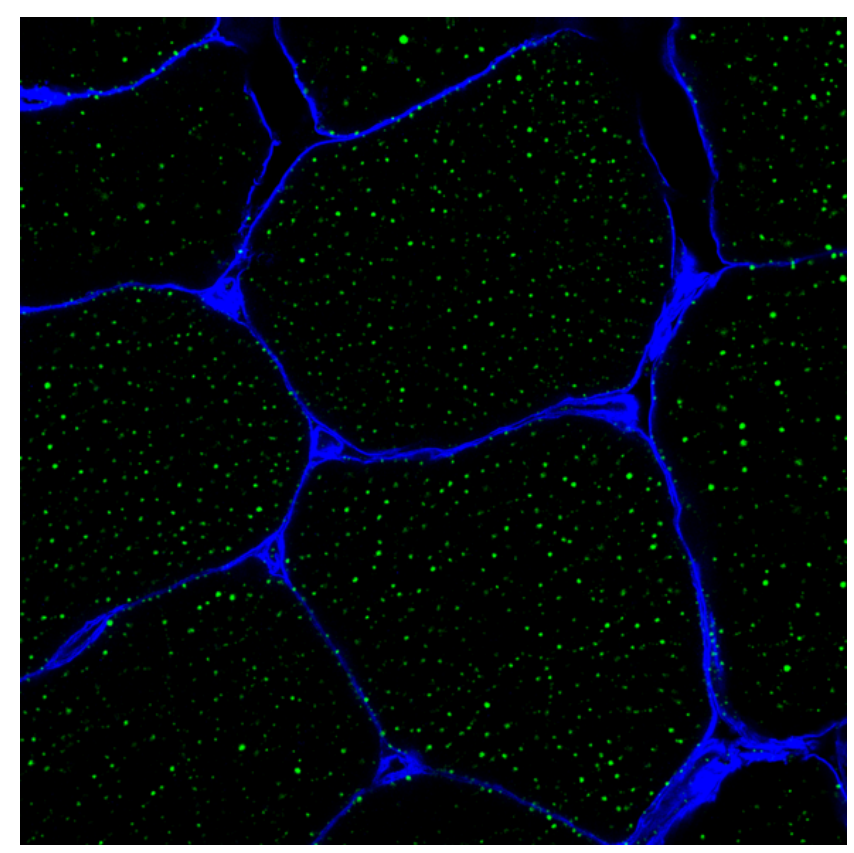

Figure 1. Intramyocellular lipids in skeletal muscle of a type 2 diabetes patient. IMCL is dispersed in small lipid droplets (stained in green). Cell membranes are shown in blue.

\section{INTRAMUSCULAR LIPID STORAGE AND INSULIN RESISTANCE}

The negative association between IMCL content and insulin sensitivity has been observed in a population consisting of sedentary lean, obese and type 2 diabetic subjects [4-6]. This association, however, is not present in endurance trained athletes. Although athletes have elevated levels of IMCL they usually are very insulin sensitive [7]. So, the relationship between lipid storage in skeletal muscle with insulin sensitivity is unlikely to be causal. This has led to the suggestion that lipid intermediates, like DAG and ceramides, are able to interfere with myocellular insulin signaling, and 
that increased IMCL is merely a surrogate measure of the presence of elevated DAG or ceramide levels.

\section{Lipid intermediates}

The higher levels of lipid intermediates in insulin resistant subjects could be a consequence of compromised fat oxidative capacity, which indeed is low in insulin resistance. This blunted oxidative capacity is due to fewer mitochondria and/or compromised mitochondrial function $[3,8]$. In combination with an elevated $I M C L$ content, mitochondrial dysfunction could result in increased availability of lipid intermediates. In contrast, endurance trained athletes have a high capacity to oxidize fat and have elevated IMCL storage [9], serving as a readily available energy source during exercise. Thus, if lipid turnover is high and fatty acids can be readily oxidized, the toxic effect of lipid intermediates is likely limited. An imbalance between adipose triglyceride lipase (ATGL) and hormone sensitive lipase (HSL) activity, which in principle could raise DAG levels, has been suggested in the insulin resistant state [10]. In cellular systems, it has been shown that these lipid intermediates interfere with insulin signaling and reduce insulin stimulated glucose uptake. Diacylglycerol inhibits the insulin signaling via PKC, which inhibits the insulin signaling cascade downstream of the insulin receptor $[11,12]$. Induction of insulin resistance in healthy lean subjects by lipid infusion resulted in an increase in DAG and PKC levels [13]. In line with this, weight loss or exercise reduces DAG content in skeletal muscle and improves insulin sensitivity in obese subjects $[14,15]$. However, changes in DAG content due to exercise interventions were not associated with improvements in insulin sensitivity. Paradoxically, athletes can have higher total DAG levels compared to obese insulin resistant subjects [9]. This suggests that total DAG in itself cannot deteriorate insulin sensitivity. However, DAG presents as 3 different isoforms, or optical isomers, with a wide range in chain length and level of saturation of the fatty acids making up the DAG pool. Particular DAG species, like saturated DAG, and DAG at specific locations in the cell are particularly potent to interfere with insulin signaling [16]. For saturated DAG content evidence is not consistent. One study shows that athletes have relatively more saturated DAG compared to lean sedentary and obese insulin resistant subjects [9]. In contrast another study showed that sedentary lean people have relatively higher levels of saturated DAG [17]. Hydrolysis of TAG in the LD will result in LD bound DAG. It should be noted though that DAG can also be of phospholipid origin and be bound to phospholipid membranes of skeletal muscle. A study showed that this membrane bound fraction of DAG makes up about $80 \%$ of the total DAG pool in muscle and is higher in insulin resistant subjects compared to endurance trained athletes. This higher membrane fraction was associated with a 
higher PKC activation and insulin resistance [18]. Especially membrane bound DAG, which is unlikely to be of lipid droplet origin, has the potential to interfere with the insulin signaling as it resides at the site where insulin signaling takes place.

Another lipid intermediate that has been associated with insulin resistance is ceramide. Ceramide is a sphingolipid and consists of one fatty acid and a sphingosine molecule. Also the fatty acids bound to ceramide can differ, but it requires palmitoyl-CoA to synthesize ceramide. Ceramide inhibits the insulin signaling pathway resulting in inhibition of glucose uptake [19]. In insulin resistant obese subjects, higher ceramide levels have been observed compared to insulin sensitive subjects $[9,20]$. In addition, ceramide levels are negatively associated with insulin sensitivity. It should be noted that differences in ceramide species or levels between groups with distinct levels of insulin sensitivity is not always reported [21]. In obese insulin resistant subjects total ceramide levels drop upon 16 weeks exercise training as an insulin-sensitizing intervention [14, 15]. However, reduced ceramide content induced by exercise training does not always associate with improvements of insulin sensitivity. Also for ceramides a subtype specific effect may underlie the variation in insulin sensitivity upon interventions altering ceramide content. Interestingly, exercise-mediated reductions in specific ceramides species (C16:0 and C24:1) were associated with improvements in insulin sensitivity $[9,15]$. These two ceramide species were higher in obese insulin resistant subjects compared to athletes [9] suggesting that these two ceramide species may interfere with insulin sensitivity.

\section{LIPID DROPLETS}

The relationship between lipid intermediates and insulin sensitivity is not straightforward. Another putative determinant of insulin sensitivity is how IMCL is stored. LDs can differ in size, can be stored at different subcellular locations, can be coated by a variety of LD coating proteins and may well be composed of different lipids. Although long be viewed as dull structures merely stalling fatty acids spill-over from excess adipose tissue lipolysis, these lipid droplets (LDs) are nowadays viewed as dynamic organelles involved in storing and releasing free fatty acids, and a variety of other processes related to cellular health [22] that are beyond the scope of this thesis. 


\section{Lipid droplet coating proteins}

LDs are surrounded by a phospholipid monolayer, which is decorated with many different coating proteins [23]. Well-studied LD coating proteins belong to the perilipin family (PLIN1 - PLIN5), see Table 1. PLIN1 is exclusively expressed in white adipose tissue, while the other perilipin family members are more ubiquitously expressed. During maturation of white adipocytes, PLIN1 is involved in the unilocular LD formation [24]. Furthermore, PLIN1 regulates lipolysis by interacting with CGI-58 and thereby preventing the activation of ATGL by CGI-58 [25]. In skeletal muscle PLIN2 is involved in TAG storage into LDs and LD stability [26]. As a consequence, lipotoxicity is prevented and skeletal muscle insulin sensitivity may be preserved. PLIN3 is associated with skeletal muscle fat oxidation $[27,28]$. In vitro studies have reported that PLIN3 is involved in lipid droplet formation $[29,30]$. About the function of PLIN4 in skeletal muscle is not much known yet. Upon training, the change in skeletal muscle PLIN4 gene expression is positively associated with the change in lipid content in the subsarcolemmal area [31]. From white adipocyte cell culture models it is known that PLIN4 coats nascent LDs during TAG synthesis [32]. PLIN5 is the most studied perilipin member in oxidative tissues, like skeletal and cardiac muscle, and is involved in the regulation LD lipolysis to match for fatty acid oxidation rates [33]. Also in the present thesis, the focus will be on PLIN5 and its putative direct or indirect involvement in modulating myocellular lipid storage and insulin sensitivity.

Table 1. Overview of the Perilipin proteins and their function in LD metabolism.

\begin{tabular}{lll}
\hline Member & Tissue & Involved in \\
\hline PLIN1 & $\begin{array}{l}\text { White adipose tissue, brown adipose } \\
\text { tissue and steroidogenic tissue }\end{array}$ & $\begin{array}{l}\text { Regulating LD lipolysis [25] } \\
\text { Formation of unilocular LD in WAT [24] }\end{array}$ \\
\hline PLIN2 & Ubiquitously & $\begin{array}{l}\text { Formation of LDs [29, 34] } \\
\text { LD stability [26] }\end{array}$ \\
\hline PLIN3 & Ubiquitously & Formation of LDs [29, 30] \\
\hline PLIN4 & $\begin{array}{l}\text { White adipose tissue, cardiac muscle } \\
\text { and skeletal muscle }\end{array}$ & Formation of LDs [32] \\
\hline PLIN5 & Oxidative tissues & Regulating LD lipolysis to match FA oxidation [33] \\
\hline
\end{tabular}

When fatty acids are required for oxidation, lipolysis of TAG occurs. In this process fatty acids are subsequently released from glycerol. Upon complete lipolysis this results in the release of three fatty acids. The first step in lipolysis is catalyzed by ATGL resulting in DAG and one fatty acid. Subsequently another fatty acid can be hydrolyzed from glycerol catalyzed by HSL. Finally, MAG is hydrolyzed by monoglyceride lipase [35]. Lipid droplet lipolysis is a complex and strictly orchestrated process with many more players like (co-)activators and suppressors involved. Important players of lipolysis in skeletal muscle beside ATGL and HSL are CGI-58, GOS2 and PLIN5. CGI-58 is a co-activator 
of ATGL and upon interaction lipolysis is stimulated [36]. Contrary to CGI-58, GOS2 inhibits ATGL activity $[37,38]$. PLIN5 regulates lipolysis, but by which mechanisms is not entirely clear yet. Several studies have shown that PLIN5 interacts with ATGL and/or CGI-58 and thereby preventing the colocalization of this lipase and its co-activator [39-41].

\section{Lipid droplet size}

It is suggested that small LD size may be a determinant of LD function, with small LD (with a high surface to volume ratio) being preferred of large LDs with a limited surface-to-volume ratio. When the surface-to-volume ratio is relatively large, more LD coating proteins per volume fat is present and lipolysis can most likely be better controlled [42]. Thus, LD size is a putative determinant of insulin sensitivity.

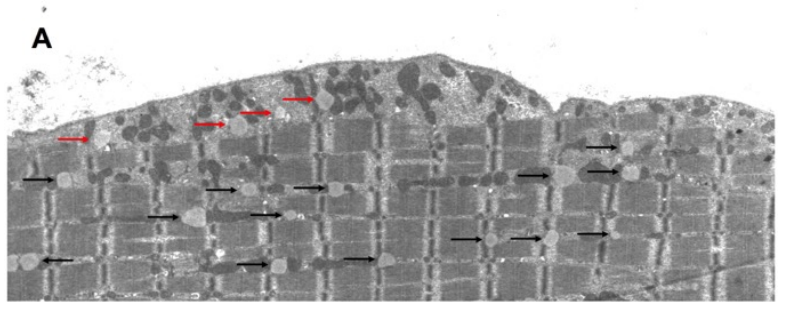

Figure 2. Subcellular location of myocellular lipid droplets. Two compartments can be distinguished (A) subsarcolemmal lipid droplets indicated by red arrows and (A and $B$ ) intermyofibrillar lipid droplets indicated by black arrows.

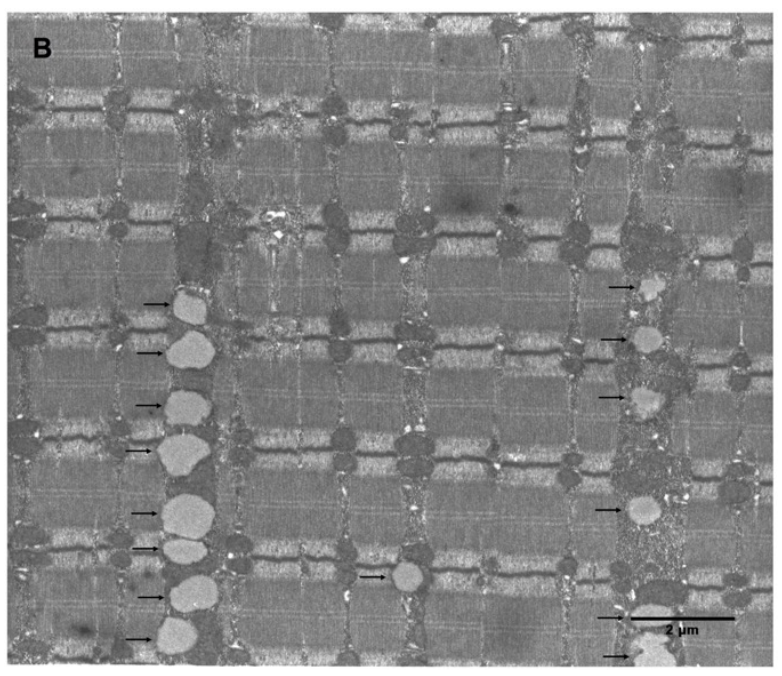

With fluorescence microscopy conflicting results are found regarding LD size between lean trained, lean sedentary, obese and type 2 diabetes subjects [43, 44]. He et al. [43] found that type 2 diabetic subjects had larger LDs compared to lean sedentary and they showed that after a 16-week exercise and dietary intervention, LD size reduced. In addition, those who reduced LD size most improved 
most in insulin sensitivity. These results unmask LD size as a potential determinant of insulin sensitivity. In contrast, van Loon et al. [44] did not observe any differences in LD size between lean trained, obese and type 2 diabetes subjects. From electron microscopy studies the picture emerges that larger LDs are related to insulin resistance [45-48].

\section{Subcellular location of myocellular lipid droplets}

Insulin signaling starts with binding of insulin to its receptor at the cellular membrane. Hence, lipid intermediates from lipid droplets close to the cell membrane might impact locally on insulin signaling. Thus, subcellular location of lipid droplets may well be of importance when it comes to insulin sensitivity. Lipid droplets can be stored subsarcolemmal, in the near vicinity of the cell membrane, or between the contractile filaments of the skeletal muscle, the intermyofibrillar lipid droplets (Figure 2). Electron microscopy images revealed that type 2 diabetic subjects disperse more lipid droplets in the subsarcolemmal region compared to obese subjects. The number of lipid droplets in the intermyofibrillar region, however, appears not to be different. Moreover, in type 2 diabetic subjects with a high number of lipid droplets in the subsarcolemmal region, the subsarcolemmal lipid droplet content correlates with insulin resistance [49]. Improving insulin sensitivity by training reduces the number of lipid droplets in the subsarcolemmal region, while the number of lipid droplets in the intermyofibrillar region is unaffected $[45,49]$. Not only the number of lipid droplets in the subsarcolemmal region is affected by training, but also lipid droplet size in the subsarcolemmal region is reduced [45]. Hence, subcellular location as well as location related alterations in LD size seem to affect insulin sensitivity.

\section{Lipid droplet composition}

The general opinion is that once lipids are stored in LDs (predominantly as triacylglycerol), these lipids are inert and will no longer be harmful to the cell. So, lipid-mediated insulin resistance can theoretically be prevented by sequestering toxic lipid intermediates in lipid droplets. Conventionally, LD composition is examined upon extracting lipids or isolating LDs from the tissue of interest. Although these techniques have clearly proven valuable in the past, these techniques cannot provide information on the lipid composition of individual LDs, nor do they take subcellular localization or coating of the lipid droplet with distinct proteins into account. Recent advances in in situ techniques like Coherent Anti-stokes Raman Spectroscopy can be used to circumvent contamination of the homogenate from lipid species present in membranes and to obtain chemical 
characteristics of the lipids in the individual LDs. Overexpression of PLIN5 in skeletal muscle promotes IMCL content and fatty acid oxidation [50] and interestingly also altered some chemical characteristics of the lipids in the cells overexpressing PLIN5 [51]. Thus, these powerful, novel and partly microscopy based tools were employed to explore the composition of individual LDs in detail in their native subcellular habitat.

\section{OUTLINE THESIS}

Research in this thesis focused on the lipid droplet characteristics in muscle tissue, mainly skeletal muscle, in relation to insulin sensitivity and the involvement of PLIN5 in modulating IMCL. In chapter 2 we examined the involvement of intramyocellular lipid handling in the differential response on insulin sensitivity upon a high-fat high-calorie diet of South Asians and Caucasians. Protein content of important players in lipid droplet dynamics and IMCL content were assessed and if any changes were related to the response on insulin sensitivity. However, to gain more insight in how lipid droplet dynamics and IMCL content are related with insulin sensitivity a microscopic approach is required. Such a fluorescence confocal microscopic approach for assessing lipid droplet morphology and their coating with PLIN5 is described in chapter 3. In addition, the added value of the superresolution technique STimulated Emission Depletion microscopy in our studies towards the putative interaction of PLIN5 with mitochondria is explored. In chapter 4 we applied fluorescence confocal microscopy to examine the role of lipid droplet characteristics like size, number, location and PLIN5 coating, in a physiological model of insulin resistance, i.e. prolonged fasting. Subsequently, we examined in chapter 5 whether the fraction of PLIN5 coated lipid droplets explain the athlete's paradox. PLIN5 overexpression results in a different chemical composition of lipid droplets. In chapter 6 we assessed in a fiber type specific manner the chemical lipid droplet composition with Coherent Anti-stokes Raman Spectroscopy in athletes and type 2 diabetic patients who differ profoundly in PLIN5 protein content. Furthermore, lipid droplet location and morphology was studied fiber type specific. The confocal fluorescence microscopy measurements I developed are being applied in chapter $\mathbf{7}$ to explore lipid droplet characteristics in biopsies from the left ventricle in metabolically non-compromised humans who underwent cardiac surgery for valve replacement. These characteristics were compared to the characteristics obtained from left ventricle biopsies obtained from obese normoglycemic and type 2 diabetes patients undergoing cardiac bypass surgery to explore the role of cardiac lipid deposition in cardiac failure. Results from previous chapters will be integrated and discussed in a broader perspective in chapter 8 . 


\section{REFERENCES}

1. Whiting, D.R., et al., IDF diabetes atlas: global estimates of the prevalence of diabetes for 2011 and 2030. Diabetes Res Clin Pract, 2011. 94(3): p. 311-21.

2. Gustafson, B., et al., Inflammation and impaired adipogenesis in hypertrophic obesity in man. Am J Physiol Endocrinol Metab, 2009. 297(5): p. E999-E1003.

3. Coen, P.M., et al., Reduced skeletal muscle oxidative capacity and elevated ceramide but not diacylglycerol content in severe obesity. Obesity (Silver Spring), 2013. 21(11): p. 2362-71.

4. Goodpaster, B.H., et al., Intramuscular lipid content is increased in obesity and decreased by weight loss. Metabolism, 2000. 49(4): p. 467-72.

5. Pan, D.A., et al., Skeletal muscle triglyceride levels are inversely related to insulin action. Diabetes, 1997. 46(6): p. 983-8.

6. Krssak, M., et al., Intramyocellular lipid concentrations are correlated with insulin sensitivity in humans: a 1 H NMR spectroscopy study. Diabetologia, 1999. 42(1): p. 113-6.

7. Goodpaster, B.H., et al., Skeletal muscle lipid content and insulin resistance: evidence for a paradox in endurance-trained athletes. J Clin Endocrinol Metab, 2001. 86(12): p. 5755-61.

8. Dela, F. and J.W. Helge, Insulin resistance and mitochondrial function in skeletal muscle. Int J Biochem Cell Biol, 2013. 45(1): p. 11-5.

9. Amati, F., et al., Skeletal muscle triglycerides, diacylglycerols, and ceramides in insulin resistance: another paradox in endurance-trained athletes? Diabetes, 2011. 60(10): p. 2588-97.

10. Badin, P.M., et al., Altered skeletal muscle lipase expression and activity contribute to insulin resistance in humans. Diabetes, 2011. 60(6): p. 1734-42.

11. Li, Y., et al., Protein kinase C Theta inhibits insulin signaling by phosphorylating IRS1 at Ser(1101). J Biol Chem, 2004. 279(44): p. 45304-7.

12. $\mathrm{Yu}, \mathrm{C}$., et al., Mechanism by which fatty acids inhibit insulin activation of insulin receptor substrate-1 (IRS-1)-associated phosphatidylinositol 3-kinase activity in muscle. J Biol Chem, 2002. 277(52): p. 50230-6.

13. Itani, S.I., et al., Lipid-induced insulin resistance in human muscle is associated with changes in diacylglycerol, protein kinase C, and IkappaB-alpha. Diabetes, 2002. 51(7): p. 2005-11.

14. Dube, J.J., et al., Exercise-induced alterations in intramyocellular lipids and insulin resistance: the athlete's paradox revisited. Am J Physiol Endocrinol Metab, 2008. 294(5): p. E882-8.

15. Dube, J.J., et al., Effects of weight loss and exercise on insulin resistance, and intramyocellular triacylglycerol, diacylglycerol and ceramide. Diabetologia, 2011. 54(5): p. 1147-56.

16. Nowotny, B., et al., Mechanisms underlying the onset of oral lipid-induced skeletal muscle insulin resistance in humans. Diabetes, 2013. 62(7): p. 2240-8.

17. Bergman, B.C., et al., Increased intramuscular lipid synthesis and low saturation relate to insulin sensitivity in endurance-trained athletes. J Appl Physiol, 2010. 108(5): p. 1134-41.

18. Bergman, B.C., et al., Localisation and composition of skeletal muscle diacylglycerol predicts insulin resistance in humans. Diabetologia, 2012. 55(4): p. 1140-50.

19. Chavez, J.A., et al., Ceramides and glucosylceramides are independent antagonists of insulin signaling. J Biol Chem, 2014. 289(2): p. 723-34.

20. Coen, P.M., et al., Insulin resistance is associated with higher intramyocellular triglycerides in type I but not type II myocytes concomitant with higher ceramide content. Diabetes, 2010. 59(1): p. 80-8.

21. Skovbro, M., et al., Human skeletal muscle ceramide content is not a major factor in muscle insulin sensitivity. Diabetologia, 2008. 51(7): p. 1253-60.

22. Welte, M.A., Expanding roles for lipid droplets. Curr Biol, 2015. 25(11): p. R470-81.

23. Walther, T.C. and R.V. Farese, Jr., The life of lipid droplets. Biochim Biophys Acta, 2009. 1791(6): p. 459-66. 
24. Grahn, T.H., et al., FSP27 and PLIN1 interaction promotes the formation of large lipid droplets in human adipocytes. Biochem Biophys Res Commun, 2013. 432(2): p. 296-301.

25. Granneman, J.G., et al., Perilipin controls lipolysis by regulating the interactions of AB-hydrolase containing 5 (Abhd5) and adipose triglyceride lipase (Atgl). J Biol Chem, 2009. 284(50): p. 34538-44.

26. Bosma, M., et al., Perilipin 2 improves insulin sensitivity in skeletal muscle despite elevated intramuscular lipid levels. Diabetes, 2012. 61(11): p. 2679-90.

27. Covington, J.D., et al., Skeletal muscle perilipin 3 and coatomer proteins are increased following exercise and are associated with fat oxidation. PLoS One, 2014. 9(3): p. e91675.

28. Covington, J.D., et al., Perilipin 3 Differentially Regulates Skeletal Muscle Lipid Oxidation in Active, Sedentary and Type 2 Diabetic Males. J Clin Endocrinol Metab, 2015: p. JC20144125.

29. Wolins, N.E., et al., S3-12, Adipophilin, and TIP47 package lipid in adipocytes. J Biol Chem, 2005. 280(19): p. 19146-55.

30. Bulankina, A.V., et al., TIP47 functions in the biogenesis of lipid droplets. J Cell Biol, 2009. 185(4): p. 641-55.

31. Pourteymour, S., et al., Perilipin 4 in human skeletal muscle: localization and effect of physical activity. Physiol Rep, 2015. 3(8).

32. Wolins, N.E., et al., Adipocyte protein S3-12 coats nascent lipid droplets. J Biol Chem, 2003. 278(39): p. 37713-21.

33. Kimmel, A.R. and C. Sztalryd, Perilipin 5, a lipid droplet protein adapted to mitochondrial energy utilization. Curr Opin Lipidol, 2014. 25(2): p. 110-7.

34. Brasaemle, D.L., et al., Adipose differentiation-related protein is an ubiquitously expressed lipid storage droplet-associated protein. J Lipid Res, 1997. 38(11): p. 2249-63.

35. Meex, R.C., P. Schrauwen, and M.K. Hesselink, Modulation of myocellular fat stores: lipid droplet dynamics in health and disease. Am J Physiol Regul Integr Comp Physiol, 2009. 297(4): p. R913-24.

36. Lass, A., et al., Adipose triglyceride lipase-mediated lipolysis of cellular fat stores is activated by CGI-58 and defective in Chanarin-Dorfman Syndrome. Cell Metab, 2006. 3(5): p. 309-19.

37. Yang, X., et al., The $G(0) / G(1)$ switch gene 2 regulates adipose lipolysis through association with adipose triglyceride lipase. Cell Metab, 2010. 11(3): p. 194-205.

38. Schweiger, M., et al., GO/G1 switch gene-2 regulates human adipocyte lipolysis by affecting activity and localization of adipose triglyceride lipase. J Lipid Res, 2012. 53(11): p. 2307-17.

39. Granneman, J.G., et al., Functional interactions between MIdp (LSDP5) and Abhd5 in the control of intracellular lipid accumulation. J Biol Chem, 2009. 284(5): p. 3049-57.

40. Granneman, J.G., et al., Interactions of perilipin-5 (Plin5) with adipose triglyceride lipase. J Biol Chem, 2011. 286(7): p. 5126-35.

41. Wang, H., et al., Unique regulation of adipose triglyceride lipase (ATGL) by perilipin 5, a lipid dropletassociated protein. J Biol Chem, 2011. 286(18): p. 15707-15.

42. Hesselink, M.K., M. Mensink, and P. Schrauwen, Intramyocellular lipids and insulin sensitivity: does size really matter? Obes Res, 2004. 12(5): p. 741-2.

43. He, J., B.H. Goodpaster, and D.E. Kelley, Effects of weight loss and physical activity on muscle lipid content and droplet size. Obes Res, 2004. 12(5): p. 761-9.

44. van Loon, L.J., et al., Intramyocellular lipid content in type 2 diabetes patients compared with overweight sedentary men and highly trained endurance athletes. Am J Physiol Endocrinol Metab, 2004. 287(3): p. E558-65.

45. Li, Y., et al., Subsarcolemmal lipid droplet responses to a combined endurance and strength exercise intervention. Physiol Rep, 2014. 2(11).

46. Fraenkel, M., et al., Scanning electron microscopic analysis of intramyocellular lipid droplets in an animal model of type 2 diabetes. Obesity (Silver Spring), 2008. 16(3): p. 695-9.

47. Molero, J.C., et al., Effects of rosiglitazone on intramyocellular lipid accumulation in Psammomys obesus. Biochim Biophys Acta, 2010. 1802(2): p. 235-9. 
48. Samjoo, I.A., et al., Markers of skeletal muscle mitochondrial function and lipid accumulation are moderately associated with the homeostasis model assessment index of insulin resistance in obese men. PLoS One, 2013. 8(6): p. e66322.

49. Nielsen, J., et al., Increased subsarcolemmal lipids in type 2 diabetes: effect of training on localization of lipids, mitochondria, and glycogen in sedentary human skeletal muscle. Am J Physiol Endocrinol Metab, 2010. 298(3): p. E706-13.

50. Bosma, M., et al., The lipid droplet coat protein perilipin 5 also localizes to muscle mitochondria. Histochem Cell Biol, 2012. 137(2): p. 205-16.

51. Billecke, N., et al., Perilipin 5 mediated lipid droplet remodelling revealed by coherent Raman imaging. Integr Biol (Camb), 2015. 


\section{CHAPTER 2}

Lipid droplet dynamics and insulin sensitivity upon a 5day high-fat diet in Caucasians and South Asians

Anne Gemmink, Leontine E.H. Bakker, Bruno Guigas, Esther Kornips, Gert

Schaart, A. Edo Meinders, Ingrid M. Jazet, Matthijs K.C. Hesselink

Published in Scientific Reports 2017; 7:42393 


\section{ABSTRACT}

A 5-day High-Fat High-Calorie diet (HFHC-diet) reduces insulin-stimulated glucose disposal (Rd) in South Asian, but not Caucasian healthy lean males. We aimed to investigate if differences in myocellular lipid handling are underlying this differential response. A two-step hyperinsulinemiceuglycemic clamp and muscle biopsies were performed in 12 healthy lean Caucasian and South Asian males (BMI<25 kg/m², 19-25 years) before and after a 5-day HFHC-diet (regular diet $+375 \mathrm{~mL}$ cream/day; $1275 \mathrm{kcal} /$ day; 94\% fat). Triglyceride extractions and Western Blots for lipid droplet and mitochondrial proteins were performed. Intramyocellular lipid content and HFHC-diet response were similar between ethnicities (group effect: $p=0.094$; diet effect: $+\sim 30 \%, p=0.044$ ). PLIN5 protein content increased upon the HFHC-diet $(P=0.031)$ and tended to be higher in South Asians $(0.87 \pm 0.42$ $\mathrm{AU}$ vs. $1.35 \pm 0.58 \mathrm{AU}, p=0.07)$. $4-\mathrm{HNE}$ tended to increase in South Asians upon the HFHC-diet (interaction effect: $p=0.057$ ). In Caucasians $\triangle P$ LIN5 content correlated with $\Delta R_{d}$ (Caucasians: $r=0.756$, $p=0.011$; South Asians: $r=-0.085, p=0.816)$, while in South Asians $\Delta 4-H N E$ associated with $\Delta$ PLIN5 content (Caucasians: $r=0.312, p=0.380$; South Asians: $r=0.771, p=0.003$ ). These data indicate that in Caucasians, PLIN5 may be protective against HFHC-diet induced insulin resistance, which for reasons not yet understood is not observed in South Asians, who possess increased lipid peroxidation levels. 


\section{INTRODUCTION}

The prevalence of type 2 diabetes is rising worldwide, more particularly in the South Asian population [1]. Compared to the Caucasian population South Asians carry a number of additional risk factors for developing type 2 diabetes. South Asians have a higher percentage body fat at a similar BMI [2] and store more of their fat in visceral adipose tissue compartments compared to Caucasians $[3,4]$.

Interestingly, at the same BMI South Asians also store more fat as intramyocellular lipids (IMCL), compared to Caucasians $[5,6]$. In the Caucasian population a negative association between IMCL content and insulin sensitivity has been reported $[7,8]$. This has led to the suggestion that lipids stored in skeletal muscle impede insulin sensitivity. However, observations in trained athletes, who also store muscle fat quite abundantly whilst being rather insulin sensitive, indicate that it is unlikely that this association is causal [9]. Fascinatingly, also in the South Asian population the relationship between IMCL content and insulin resistance is not observed $[5,10]$.

These observations in two phenotypically different populations indicate that the amount of IMCL is not necessarily a determinant of insulin sensitivity. Rather, the capacity of the IMCL pool to undergo continuous release and storage of fat in order to meet changes in demand and supply, a process referred to as lipid droplet (LD) dynamics, might be of more importance for insulin sensitivity [11] than IMCL content per se. Thus in a state of reduced LD dynamics, IMCL may impede insulin sensitivity more profoundly. Although mitochondrial content $[3,12]$ and function $[6]$ in South Asians are similar or even higher compared to Caucasians, fat oxidative capacity during submaximal exercise is lower in South Asians than in Caucasians [3]. This observation may indicate that release of fatty acids to fuel oxidation in South Asians may be compromised and that LDs in skeletal muscle of South Asians are less dynamic than in Caucasians.

Recognized players in skeletal muscle LD dynamics are proteins from the perilipin family (PLIN1-5), the major adipose triacylglycerol lipase ATGL [13] and its co-activator CGI-58[14]. While ATGL and CGI-58 jointly stimulate LD lipolysis, PLIN5 regulates LD lipolysis by interacting with ATGL and CGI58[15] to facilitate fat oxidation. Insulin sensitive endurance trained athletes, recognized for the highly dynamic nature of their LD pool, possess higher expression of PLIN2, PLIN5 and ATGL compared to lean untrained individuals [16]. In addition, training induces ATGL, PLIN3 and PLIN5 protein expression in obese subjects [17]. Upon consumption of a high fat diet, IMCL content increases in rodents while ATGL drops [18] and expression of PLIN5 [18, 19] and CGI-58 increases 
[18], also indicative of alterations in LD dynamics. Thus, both insulin sensitizing and desensitizing interventions affect proteins involved in LD dynamics in skeletal muscle.

We recently published that upon a 5-day high-fat, high-calorie diet (HFHC-diet) South Asians develop peripheral insulin resistance, while Caucasians remain insulin sensitive [12]. Increases in IMCL content with reduced insulin sensitivity have been reported upon short term high-fat diets up to 3 days $[20,21]$. When high-fat diets are maintained for a longer period, the effects on IMCL and insulin sensitivity are less prominent, if not absent [22-25]. Here we used the 5-day HFHC-diet approach to examine the role of IMCL and LD dynamics in the differential response in insulin sensitivity of Caucasians and South Asians. We hypothesized that Caucasians are better capable to maintain insulin sensitivity upon a lipid overload due to differences in LD dynamics than South Asians. To test this hypothesis, we analyzed muscle biopsies of South Asians and Caucasians on IMCL content and expression of proteins involved in LD dynamics before and after the 5-day HFHC-diet.

\section{METHODS}

\section{Ethic statement}

The Medical Ethic Committee of Leiden University Medical Center approved this study. The study was performed according the principles of the revised Declaration of Helsinki. All volunteers gave written informed consent before participation. The present study represents a detailed analysis of new parameters assessed in samples derived from a previously published paper [12].

\section{Subjects and study protocol}

Twelve healthy lean Caucasian and twelve healthy lean South Asian males aged between 19 and 25 years were included. The study protocol was described previously [12]. In short, all subjects followed for 5 days a HFHC-diet consisting of their regular diet with the addition of 3 cups of $125 \mathrm{ml}$ cream per day (1275 kcal/day, 94\% fat). Before and after the diet a muscle biopsy was taken from the $m$. vastus lateralis with a modified Bergström needle [39], and a 2-step hyperinsulinemic-euglycemic clamp and indirect calorimetry with a ventilated hood (Oxycon Pro, CareFusion, Höchberg, Germany) were performed. The indirect calorimetry was performed during basal conditions and during both steps of the hyperinsulinemic-euglycemic clamp (for detailed data see [12]). 


\section{Triacylglycerol content}

Triacylglycerol (TAG) was biochemically extracted according to Schwartz and Wolins [40] from 5-10 mg muscle tissue. TAG content was corrected for protein concentration, which was determined with the DC kit (Bio-Rad, Veenendaal, The Netherlands).

\section{Western blotting}

Western blots were performed with primary antibodies against ATGL (2138, Cell Signalling Technology, Bioké, Leiden, The Netherlands), CGI-58 (NB110-41576, Novus Biologicals, Littleton, Colorado, USA), PLIN2 (GP40, Progen Biotechnik, Heidelberg, Germany), PLIN3 (M6PRBP1, Acris, Herford, Germany), PLIN5 (GP31, Progen Biotechnik, Heidelberg, Germany), 4-HNE (393207, Calbiochem, VWR International BV, Amsterdam, The Netherlands), PGC-1 $\alpha$ (516557, Calbiochem, VWR International BV, Amsterdam, The Netherlands) and an antibody cocktail against distinct structural proteins of the 5 individual mitochondrial electron transport chain complexes (OXPHOS ab110411, Abcam, Cambridge, UK). For ATGL, PLIN2, PLIN5 and OXPHOS detection IRDye700conjugated or IRDye800-conjugated secondary antibodies were used and visualized with the Odyssey infrared detector (LI-COR Biosciences, Westburg, Leusden, The Netherlands). An HRPconjugated secondary antibody was used for CGI-58 and detected with ECL. For all antibodies the molecular weight in the gel was checked with known molecular weight and positive and/or negative controls are applied to assess the validity of the band selected for quantification. In the current study we have used HEK cells overexpressing PLIN2 or PLN5 to identify the appropriate band. For PLIN3 we have a synthetic peptide that we run on the same gels to identify band height and for ATGL we used tissue of ATGL ko mice and a patient with a compound ATGL mutation [41] to identify the right band. For CGI-58 we combined the information of the data sheet of the supplier with data from fibroblasts of a CGI58-deficient patient [14]. For PGC1- $\alpha$ tissue from a PGC1- $\alpha$ transgenic mice was used as a positive control. For valid loading of equal amounts of muscle protein, a three step approach is used; an instant blue gel is loaded allowing us to ensure that we indeed load uncontaminated muscle tissue. Next, this gel is scanned for optical density to calculate how much of the lysate we need to load to a new gel to obtain equal amounts of protein. Thus, the gel loaded for Western blotting has the same amount of protein loaded to each and every lane and is a valid reflection of a comparable amount of muscle protein. To check for efficient electro transfer of the gels to the membrane, we included the data for the housekeeping protein $\alpha$-SR-actin (A2172, Sigma, St. Louis, USA) or GAPDH (2118, Cell Signaling Technology, Bioké, Leiden, The Netherlands) as 


\section{CHAPTER 2}

reference. For valid inter-blot comparisons, we used the background correction option in the software of the supplier (Image Studio ${ }^{\text {TM }}$ Software for the Odyssey CLx - LI-COR Biosciences) and scanned the corresponding band of the protein of interest for all lanes on the blot and compute the mean of the relevant bands. Finally, we compute the ratio of the optical density of individual protein bands over the mean of the proteins bands in that particular blot.

\section{Statistics}

Results are presented as mean \pm SEM. Statistical testing was performed with SPSS version 21.0 (SPSS, Chicago, IL, USA). A repeated measures ANOVA with diet as a within factor and group as between factor was performed to test for statistical significant differences between groups and diet effect. When appropriate, a Bonferroni post-hoc test was performed. Pearson correlations coefficients were used to describe the linear association between variables. $p<0.05$ was considered statistically significant.

\section{RESULTS}

\section{Subject characteristics}

The subject characteristics were previously reported elsewhere [12]. In short, at baseline South Asians had a higher fat mass, $\mathrm{HbA}_{1 c}$ and higher insulin levels during the clamp than Caucasians. Metabolic flexibility and peripheral insulin sensitivity (Rd) was similar for both ethnicities at baseline. The 5-day HFHC-diet induced a very modest weight gain in both groups (Caucasians: from $75.1 \pm 1.8$ to $75.6 \pm 1.8 \mathrm{~kg}$, not significant; South Asians: from $63.2 \pm 2.3$ to $63.7 \pm 2.3 \mathrm{~kg}, p<0.05$ ) and insulin resistance in South Asians, but not in Caucasian subjects (Caucasians: from $41.7 \pm 2.9$ to $41.0 \pm 2.8$ $\mu \mathrm{mol} / \mathrm{kg}_{\mathrm{LBM}} / \mathrm{min}$, not significant; South Asians: from $48.7 \pm 2.9$ to $39.0 \pm 2.1 \mu \mathrm{mol} / \mathrm{kg}_{\mathrm{LBM}} / \mathrm{min}, p<0.005$ ) [12].

PLIN5 protein content associates with peripheral insulin sensitivity in Caucasians and not in South Asians at baseline

We examined baseline IMCL content and protein expression of ATGL, CGI-58, PLIN2, PLIN3 and PLIN5 to assess putative differences between Caucasians and South Asians. As shown in Figure 1, 
baseline IMCL content was not significantly different between ethnicities $(0.008 \pm 0.003$ vs. $0.015 \pm 0.003 \mu \mathrm{g} / \mu \mathrm{g}$ protein for Caucasians and South Asians, respectively). Furthermore, protein contents of ATGL, CGI-58, PLIN2, PLIN3 and PLIN5 were comparable in Caucasians and South Asians (ATGL: $1.00 \pm 0.15$ vs. $0.84 \pm 0.11 \mathrm{AU}, \mathrm{CGI}-58$ : $0.91 \pm 0.14$ vs. $1.03 \pm 0.15 \mathrm{AU}$, PLIN2: $0.98 \pm 0.07$ vs.1.00 $\pm 0.09 \mathrm{AU}$, PLIN3: $1.00 \pm 0.12$ vs. $0.84 \pm 0.07 \mathrm{AU}$, PLIN5: $0.67 \pm 0.11$ vs. $0.95 \pm 0.16 \mathrm{AU}$ for Caucasians vs. South Asians; Figure 2).

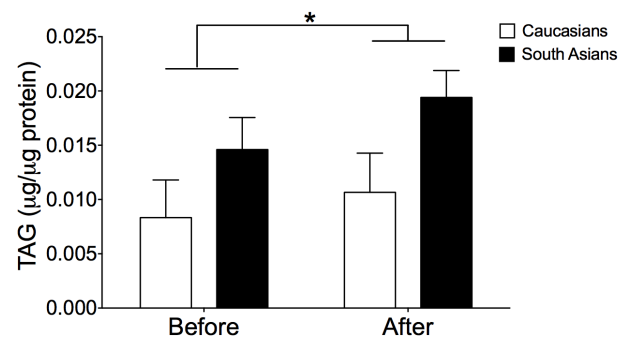

Figure 1. Intramyocellular lipid content (triacylglycerol, TAG) in Caucasian and South Asian subjects before and after a 5-day HFHC-diet. Data are presented as mean \pm SEM and were statistically analyzed with a Repeated Measures ANOVA; * $p<0.05$ for diet effect.

Of these proteins, PLIN5 has been associated with changes in (markers of) lipid-related insulin resistance [26-28]. Given the fairly wide range in insulin sensitivity within the groups, we anticipated that correlation analysis of PLIN5 with Rd could yield relevant information, even though the primary aim of the study was the comparison between Caucasians and South Asians. Thus, we assessed whether baseline IMCL content and protein levels of ATGL, CGI-58, PLIN2, PLIN3 and PLIN5 correlated with Rd. IMCL, ATGL, PLIN2 and PLIN3 content did not correlate with Rd, neither in Caucasians nor in South Asians (data not shown). In South Asians, however, CGI-58 correlated positively with $\mathrm{Rd}(r=0.749, p=0.013)$ whereas no such correlation was observed in Caucasians ( $r=-$ 0.186, $p=0.585)$. Interestingly, PLIN5 also correlated positively with Rd in Caucasian subjects, but not in South Asian subjects (Table 1). In support of this association we observed positive correlations between PLIN5 and other surrogate markers of insulin sensitivity (insulin stimulated RQ, metabolic flexibility, insulin-stimulated glucose oxidation and insulin stimulated NOGD, Table 1 and Supplementary Fig. S1). 
A

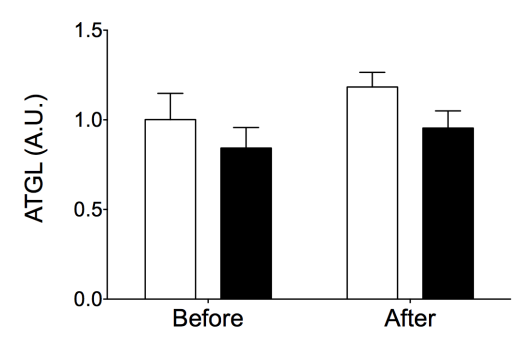

ATGL

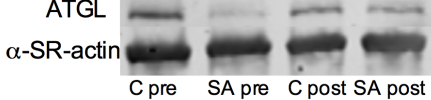

B

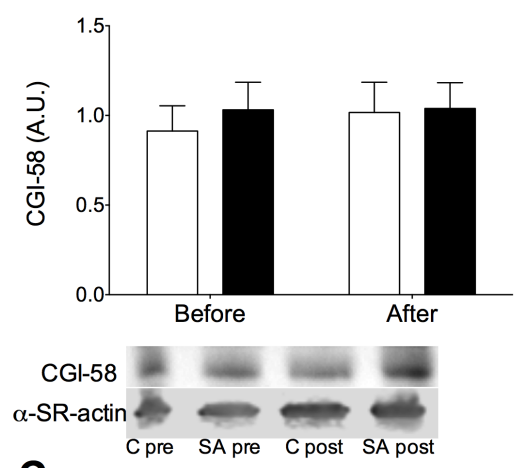

C

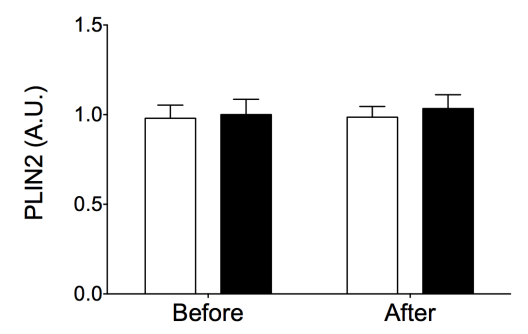

PLIN2
D

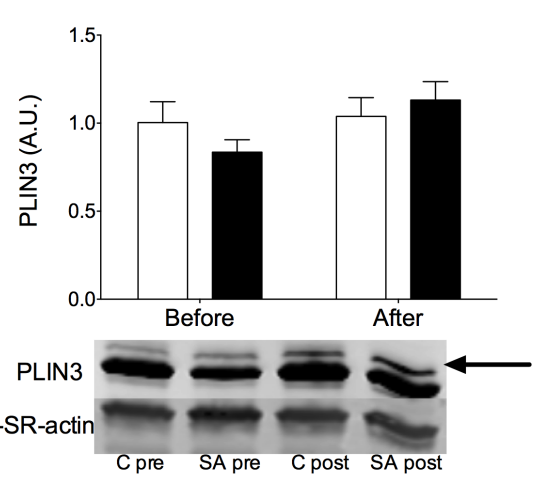

E

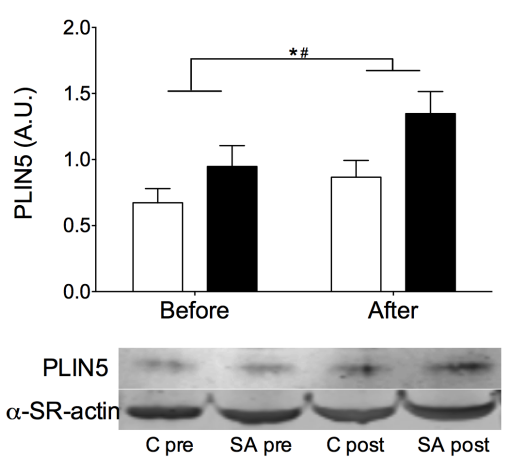

Figure 2. Protein content of important players involved in lipid droplet dynamics in Caucasian and South Asian subjects before and after a 5 day HFHC-diet. (A) ATGL, (B) CGI-58, (C) PLIN2, (D) PLIN3 and (E) PLIN5 protein content. Data are presented as mean \pm SEM and were statistically analyzed with a Repeated Measures ANOVA; * $p<0.05$ for diet effect, $\# p<0.05$ for group effect.

\section{Response of IMCL and PLIN5 to a 5-day HFHC-diet}

We previously reported that the 5-day HFHC-diet induced insulin resistance in South Asians, whereas Caucasians were capable of maintaining peripheral insulin sensitivity [12]. This differential response of insulin sensitivity to a HFHC-diet might be due to a different response of IMCL and/or lipid droplet coating proteins to the HFHC-diet.

Although we did not observe a significant interaction effect $(p=0.456)$ for IMCL content, there was a significant diet effect $(P=0.044)$ with a tendency for a group effect $(p=0.094)$. In both ethnicities IMCL content increased $\sim 30 \%$ upon the HFHC-diet (Figure 1). While protein contents of ATGL, CGI-58 and PLIN2 were unaffected by the HFHC-diet and did not differ between groups after the diet (Figure 2), a tendency for an increase in PLIN3 protein content was observed (diet effect, $p=0.074$ ). For PLIN5, 
however, we observed a significant group effect $(p=0.027)$, which tended to be higher after the HFHC-diet in the South Asians $(0.87 \pm 0.13 \mathrm{AU}$ and $1.35 \pm 0.17 \mathrm{AU}$ for Caucasians and South Asians respectively, $p=0.07)$. We observed a diet effect $(p=0.031)$ for PLIN5 as well, with a comparable dietinduced increase in PLIN5 for both groups (23\% for Caucasians and $49 \%$ for South Asians, $p=0.430$ ).

Table 1. Correlations between PLIN5 protein content and markers of insulin sensitivity in Caucasian and South Asian subjects before and after a 5-day HFHC-diet

\begin{tabular}{|c|c|c|c|c|c|c|c|}
\hline & & & Before & & & After & \\
\hline & & All & Caucasians & South Asians & All & Caucasians & South Asians \\
\hline \multirow[t]{3}{*}{$\mathrm{R}_{\mathrm{d}}\left(\mu \mathrm{mol} * \mathrm{~L} / \mathrm{Kg}_{\mathrm{LBM}} / \mathrm{min} / \mathrm{mU}\right)$} & $r$ & 0.610 & 0.895 & 0.172 & 0.257 & 0.668 & 0.084 \\
\hline & $p$ & 0.004 & $<0.001$ & 0.635 & 0.275 & 0.035 & 0.818 \\
\hline & $\mathrm{n}$ & 20 & 10 & 10 & 20 & 10 & 10 \\
\hline \multirow[t]{3}{*}{ RQ basal } & $r$ & 0.234 & -0.031 & 0.442 & 0.482 & 0.680 & 0.391 \\
\hline & $p$ & 0.295 & 0.929 & 0.174 & 0.027 & 0.030 & 0.235 \\
\hline & $\mathrm{n}$ & 22 & 11 & 11 & 21 & 10 & 11 \\
\hline \multirow[t]{3}{*}{$\mathrm{RQ}$ insulin stimulated } & $r$ & 0.405 & 0.618 & 0.328 & 0.446 & 0.322 & 0.416 \\
\hline & $p$ & 0.076 & 0.043 & 0.389 & 0.049 & 0.398 & 0.203 \\
\hline & $\mathrm{n}$ & 20 & 11 & 9 & 20 & 9 & 11 \\
\hline \multirow[t]{3}{*}{ Metabolic flexibility } & $r$ & 0.472 & 0.856 & 0.013 & 0.103 & -0.305 & 0.187 \\
\hline & $p$ & 0.035 & 0.001 & 0.974 & 0.666 & 0.424 & 0.582 \\
\hline & $\mathrm{n}$ & 20 & 11 & 9 & 20 & 9 & 11 \\
\hline \multirow[t]{3}{*}{ Lipid oxidation } & $r$ & -0.230 & 0.137 & -0.481 & -0.419 & -0.684 & -0.337 \\
\hline & $p$ & 0.302 & 0.687 & 0.134 & 0.059 & 0.029 & 0.311 \\
\hline & $\mathrm{n}$ & 22 & 11 & 11 & 21 & 10 & 11 \\
\hline \multirow{3}{*}{$\begin{array}{l}\text { Insulin stimulated glucose } \\
\text { oxidation }\left(\mu \mathrm{mol} / \mathrm{kg}_{\mathrm{LBM}} / \mathrm{min}\right)\end{array}$} & $r$ & 0.327 & 0.750 & -0.085 & -0.109 & -0.233 & -0.090 \\
\hline & $p$ & 0.159 & 0.008 & 0.828 & 0.648 & 0.547 & 0.791 \\
\hline & $\mathrm{n}$ & 20 & 11 & 9 & 20 & 9 & 11 \\
\hline \multirow{3}{*}{$\begin{array}{l}\text { Insulin stimulated NOGD } \\
\left(\mu \mathrm{mol} / \mathrm{kg}_{\text {LBM }} / \mathrm{min}\right)\end{array}$} & $r$ & 0.482 & 0.792 & 0.151 & 0.134 & 0.817 & -0.030 \\
\hline & $p$ & 0.032 & 0.004 & 0.698 & 0.572 & 0.007 & 0.930 \\
\hline & $\mathrm{n}$ & 20 & 11 & 9 & 20 & 9 & 11 \\
\hline
\end{tabular}

\section{PLIN5 correlates with insulin sensitivity in Caucasians after the 5-day HFHC-diet}

As we observed a positive correlation between PLIN5 content and markers of insulin sensitivity at baseline in the Caucasian subjects, we also explored if this correlation was maintained after consumption of the HFHC-diet. In line with the observation prior to the HFHC-diet, IMCL content was not associated with Rd after the diet, irrespective of ethnicity $(r=-0.247, p=0.522$ for Caucasians and $r=-0.408, p=0.213$ for South Asians). In addition, the HFHC-diet induced change in IMCL content did not correlate with the change in $\mathrm{Rd}(r=-0.064, p=0.918$ for Caucasians and $r=-0.517, p=0.190$ for South Asians). 
Similar to our observations before the HFHC-diet, PLIN5 protein content correlated positively with Rd and insulin-stimulated NOGD in Caucasians (Table 1 and Supplementary Fig. S2), but not in South Asians after the diet. Interestingly, when we correlated the change in PLIN5 content with the changes in Rd we did not observe a correlation for all subjects together (South Asians and Caucasians) (Figure 3A, $r=-0.078, p=0.744$ ). However, in the Caucasian participants we did observe a positive correlation between the change in PLIN5 content and the change in Rd (Figure 3B, $r=0.756$, $p=0.011$ ), whereas no correlation was found in South Asians (Figure 3C, $r=-0.085 ; p=0.816$ ).

A

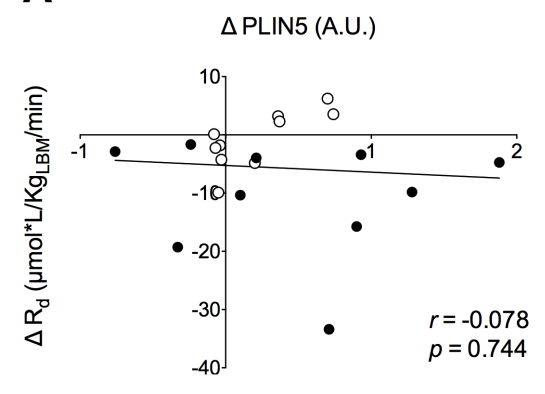

B

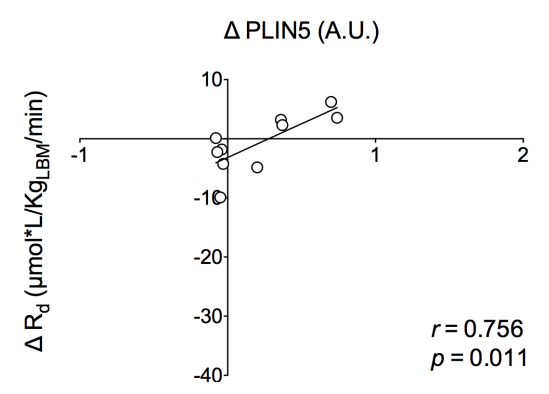

C

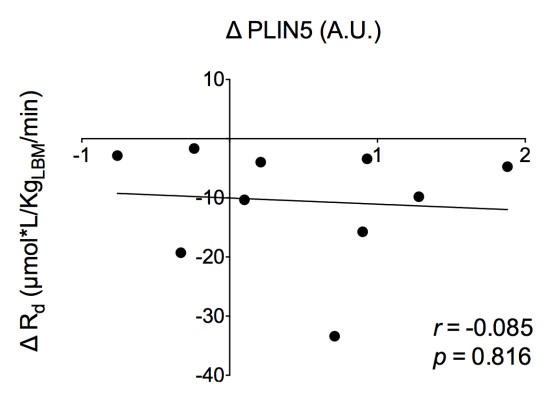

Figure 3. Correlations between the HFHC-diet induced changes in PLIN5 protein content and glucose disposal rate. (A) All participants, (B) Caucasian participants and (C) South Asian participants. Pearson correlation coefficients were used to describe the linear associations; Caucasians: 0 , South Asians: $\bullet$ 


\section{Mitochondrial content and lipid peroxidation}

In previous studies high PLIN5 protein levels have been associated with higher levels of insulin sensitivity $[16,26]$ and was reportedly to be protective against development of insulin resistance $[27,28]$. It is hence remarkable that South Asians developed insulin resistance upon a HFHC-diet, despite having higher levels of PLIN5 protein than Caucasians. PLIN5 is suggested to regulate LD dynamics for oxidative lipolysis. Thus, if fatty acids are released from the LD under conditions of compromised mitochondrial biogenesis or content, lipotoxicity and subsequently insulin resistance may develop. To explore this hypothesis, we measured marker proteins of mitochondrial biogenesis (PGC-1 $\alpha$ ) and content (various ETC complex subunits) along with a marker of lipid peroxidation, 4hydroxynonenal (4-HNE). PGC-1 $\alpha$ protein content was similar for South Asians and Caucasians before and after the HFHC-diet and was unaffected by the HFHC-diet (Figure 4A). Mitochondrial content measured as total content of 5 different ETC complex subunits was similar between ethnicities at baseline and tended to increase mainly in the Caucasian subjects (diet effect $p=0.075$ )

A

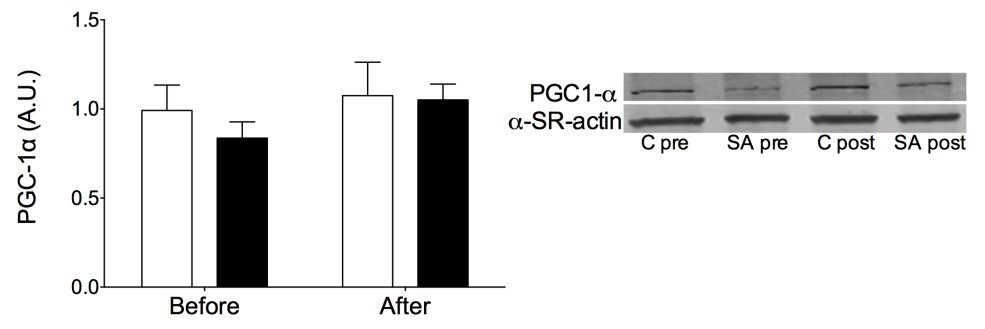

B

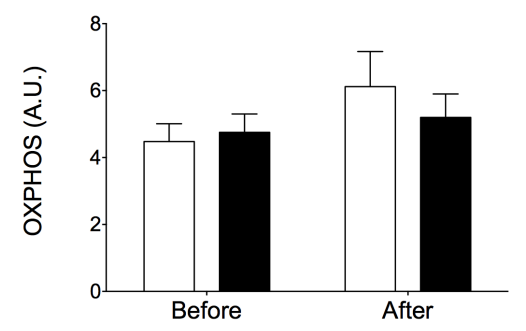

C

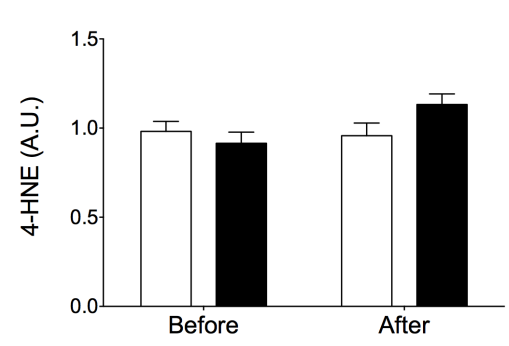

Caucasians

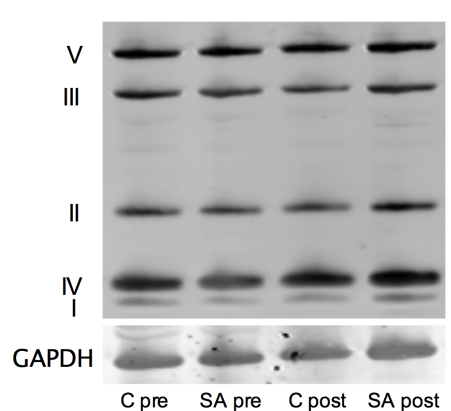

C pre SA pre C post SA post

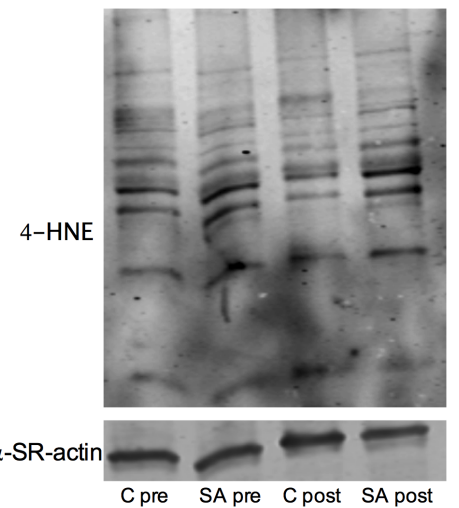

Figure 4. Mitochondrial content and lipotoxicity. Mitochondrial biogenesis (A), mitochondrial content measured as the total content of all 5 complexes (B), and levels of lipid peroxidation determined by 4-HNE (C) in Caucasian and South Asian subjects before and after a 5-day HFHC-diet. Data are presented as mean \pm SEM and were statistically analyzed with a Repeated Measures ANOVA. 
(Figure 4B). Both the pattern of lipid peroxidation as well as total 4-HNE content (Figure 4C) was similar between ethnicities at baseline, whereas a tendency for an interaction effect $(p=0.057)$ was observed for 4-HNE content. Indeed, skeletal muscle 4-HNE content was unaltered in Caucasians upon the HFHC-diet whereas it tended to increase in South Asians. In addition, this increase in 4-HNE correlated positively with the increase in PLIN5 protein content in South Asians ( $r=0.771, p=0.003)$, but not in the Caucasians $(r=0.312, p=0.380)$ (Figure 5).
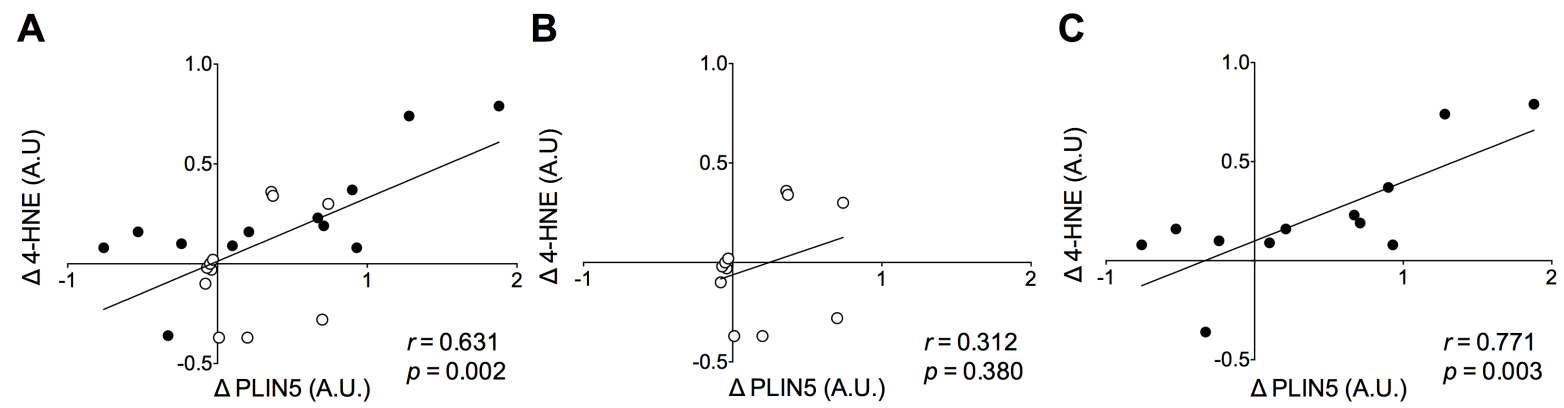

Figure 5. Correlations between the HFHC-diet induced changes in PLIN5 protein content and lipid peroxidation levels. (A) All participants, (B) Caucasian participants and (C) South Asian participants. Pearson correlation coefficients were used to describe the linear associations; Caucasians: O, South Asians: $\bullet$.

\section{DISCUSSION}

In the present study we aimed to examine whether the differential response on peripheral insulin sensitivity to a 5-day HFHC-diet between subjects from Caucasian and South Asian origin could be explained by differences in players involved in myocellular lipid handling. Although IMCL content increased similarly in both groups upon the HFHC-diet, subjects from South Asian origin possess more severe insulin resistance than Caucasians. None of the recognized players in LD dynamics (ATGL, CGI-58, PLIN2 and PLIN3) were significantly different between ethnicities at baseline, nor did they respond differentially to the HFHC-diet. So the more profound insulin resistance observed in South Asians could not be attributed to differences in LD dynamics. Surprisingly, the LD coat protein PLIN5, which is most commonly associated with improvements in insulin sensitivity or protection against lipid-induced insulin resistance, was found to be increased upon the HFHC-diet in both groups with a more robust effect in (the more insulin resistant) South Asians. The change in PLIN5 protein content correlated with the change in Rd in Caucasians. This matches the notion that PLIN5 might be considered as a LD coat protein associated with benign myocellular lipid storage. For reasons yet unknown, this correlation was absent in South Asians. 
Whereas South Asians and Caucasians both increased IMCL content upon a 5-day HFHC-diet, only the South Asians became insulin resistant [12]. This may indicate that handling of excess lipids in South Asians may differ from Caucasians. Myocellular lipids are stored in LDs which are currently recognized as dynamic organelles that can readily switch between release and storage of fatty acids upon changes in substrate availability and demand. Release of fatty acids from myocellular LDs serves a dual role: fueling mitochondrial oxidation and/or acting as ligands for PPAR $\alpha$ - and PGC1 $\alpha$ mediated changes in oxidative gene expression and mitochondrial biogenesis [16, 29, 30]. Liberation of fatty acids from the LD depends on the activation of ATGL [13], partly via interaction with its cofactor CGI-58 [14] and PLIN5[31]. In muscle cells, PLIN5 binds to GCI-58 to suppress basal lipolysis $[32,33]$. Activation of lipolysis involves dissociation of PLIN5 and CGI-58, making CGI-58 available for interaction with ATGL to stimulate its lipolytic activity. Endogenously available long-chain Acyl-CoA esters can stimulate the association of PLIN5 and CGI-58 and thus prevents binding of CGI-58 with ATGL, hence preventing lipolysis [34]. Thus, the lipolytic rate of PLIN5-coated LDs can be tuned to substrate availability and demand, and levels of LD-derived insulin desensitizing lipids can be wellcontrolled, providing a mechanistic explanation on how PLIN5 can modulate lipid-induced insulin resistance. In accordance with this role of PLIN5, we previously observed that upon prolonged fasting PLIN5 was protective against fasting-induced insulin resistance [28]. Our current observation in Caucasians that those who increase PLIN5 content the most dropped the least in insulin sensitivity upon the HFHC-diet, matches the notion that PLIN5 may be protective.

No such correlation, however, was observed in participants from South Asian origin, who became more insulin resistant after the HFHC-diet, despite a significant increase in skeletal muscle PLIN5 content. This suggests that the previously hypothesized role of PLIN5 in prevention of insulin resistance $[15,28,35]$ may operate in Caucasians, whereas in the present study, this was not observed in South Asians. This apparent discrepancy between Caucasian and South Asian subjects in dealing with excess lipids may originate from differences in mitochondrial lipid handling. Thus, we examined markers of fat oxidative capacity and observed that at the whole body level, rates of lipid oxidation under basal as well as under insulin-stimulated conditions were comparable between ethnicities [12]. Markers of muscle mitochondrial biogenesis (PGC1- $\alpha$ ) or mitochondrial content also revealed no significant differences between Caucasians and South Asians. Although this is in line with previous reports in literature $[3,6]$, it does not provide an explanation for the observed discrepancy. 
It has previously been observed that in the obese state a higher fraction of IMCL is present as peroxidized lipids (4-hydroxynonenal (4-HNE)) [36], a lipid-derived reactive aldehyde with proven insulin-desensitizing properties [37]. Indeed, increased 4-HNE protein adducts have been observed in skeletal muscle of insulin resistant humans [38]. Interestingly, we also observed a strong tendency for a differential response in 4-HNE levels in South Asians compared to Caucasians, with higher levels of 4-HNE in South Asians upon consumption of a HFHC-diet. In the present study, however, the increase in 4-HNE did not correlate with the changes in insulin-stimulated glucose uptake in any of the groups. Rather, 4-HNE correlated positively with the increase in PLIN5 in South Asians and not in Caucasians. Whether this correlation merely reflects that induction of PLIN5 is paralleled by increases in IMCL (hence rendering the opportunity for more lipids to be subject to peroxidation) or that it is a reflection of a more complex and indirect interaction remains to be elucidated.

In conclusion, the present study shows that despite similar increases in IMCL content, insulinstimulated glucose uptake in subjects from South Asian origin is compromised more profoundly than in subjects from Caucasian origin. In contrast to our hypothesis, this differential response does not seem to originate from differences in proteins involved in lipid droplet dynamics or fat oxidative capacity. In Caucasians, the LD coat protein PLIN5, which is involved in regulating oxidative lipolysis, seems to be protective against HFHC diet-induced insulin resistance. For reasons not yet known, this potential protective effect of PLIN5 is not observed in South Asians, who do possess higher myocellular levels of insulin desensitizing lipid peroxidation products than Caucasians.

\section{REFEENCES}

1. Whiting, D.R., et al., IDF diabetes atlas: global estimates of the prevalence of diabetes for 2011 and 2030. Diabetes Res Clin Pract, 2011. 94(3): p. 311-21.

2. Chandalia, M., et al., Insulin resistance and body fat distribution in South Asian men compared to Caucasian men. PLoS One, 2007. 2(8): p. e812.

3. Hall, L.M., et al., Fat oxidation, fitness and skeletal muscle expression of oxidative/lipid metabolism genes in South Asians: implications for insulin resistance? PLoS One, 2010. 5(12): p. e14197.

4. Lear, S.A., et al., Elevation in cardiovascular disease risk in South Asians is mediated by differences in visceral adipose tissue. Obesity (Silver Spring), 2012. 20(6): p. 1293-300.

5. Forouhi, N.G., et al., Relation of triglyceride stores in skeletal muscle cells to central obesity and insulin sensitivity in European and South Asian men. Diabetologia, 1999. 42(8): p. 932-5.

6. Nair, K.S., et al., Asian Indians have enhanced skeletal muscle mitochondrial capacity to produce ATP in association with severe insulin resistance. Diabetes, 2008. 57(5): p. 1166-75.

7. Krssak, M., et al., Intramyocellular lipid concentrations are correlated with insulin sensitivity in humans: a 1 H NMR spectroscopy study. Diabetologia, 1999. 42(1): p. 113-6.

8. Goodpaster, B.H., et al., Intramuscular lipid content is increased in obesity and decreased by weight loss. Metabolism, 2000. 49(4): p. 467-72. 
9. Goodpaster, B.H., et al., Skeletal muscle lipid content and insulin resistance: evidence for a paradox in endurance-trained athletes. J Clin Endocrinol Metab, 2001. 86(12): p. 5755-61.

10. Sinha, S., et al., Subclinical inflammation and soleus muscle intramyocellular lipids in healthy Asian Indian males. Clin Endocrinol (Oxf), 2005. 63(3): p. 350-5.

11. Bosma, M., et al., Re-evaluating lipotoxic triggers in skeletal muscle: relating intramyocellular lipid metabolism to insulin sensitivity. Prog Lipid Res, 2012. 51(1): p. 36-49.

12. Bakker, L.E., et al., A 5-day high-fat, high-calorie diet impairs insulin sensitivity in healthy, young South Asian men but not in Caucasian men. Diabetes, 2014. 63(1): p. 248-58.

13. Zimmermann, R., et al., Fat mobilization in adipose tissue is promoted by adipose triglyceride lipase. Science, 2004. 306(5700): p. 1383-6.

14. Lass, A., et al., Adipose triglyceride lipase-mediated lipolysis of cellular fat stores is activated by CGI-58 and defective in Chanarin-Dorfman Syndrome. Cell Metab, 2006. 3(5): p. 309-19.

15. Kimmel, A.R. and C. Sztalryd, Perilipin 5, a lipid droplet protein adapted to mitochondrial energy utilization. Curr Opin Lipidol, 2014. 25(2): p. 110-7.

16. Koves, T.R., et al., PPARgamma coactivator-1alpha contributes to exercise-induced regulation of intramuscular lipid droplet programming in mice and humans. J Lipid Res, 2013. 54(2): p. 522-34.

17. Louche, K., et al., Endurance exercise training up-regulates lipolytic proteins and reduces triglyceride content in skeletal muscle of obese subjects. J Clin Endocrinol Metab, 2013. 98(12): p. 4863-71.

18. Badin, P.M., et al., High-fat diet-mediated lipotoxicity and insulin resistance is related to impaired lipase expression in mouse skeletal muscle. Endocrinology, 2013. 154(4): p. 1444-53.

19. Rinnankoski-Tuikka, R., et al., Lipid droplet-associated proteins in high-fat fed mice with the effects of voluntary running and diet change. Metabolism, 2014. 63(8): p. 1031-40.

20. Pehleman, T.L., et al., Enzymatic regulation of glucose disposal in human skeletal muscle after a highfat, low-carbohydrate diet. J Appl Physiol (1985), 2005. 98(1): p. 100-7.

21. Bachmann, O.P., et al., Effects of intravenous and dietary lipid challenge on intramyocellular lipid content and the relation with insulin sensitivity in humans. Diabetes, 2001. 50(11): p. 2579-84.

22. Cutler, D.L., et al., Low-carbohydrate diet alters intracellular glucose metabolism but not overall glucose disposal in exercise-trained subjects. Metabolism, 1995. 44(10): p. 1264-70.

23. Chokkalingam, K., et al., High-fat/low-carbohydrate diet reduces insulin-stimulated carbohydrate oxidation but stimulates nonoxidative glucose disposal in humans: An important role for skeletal muscle pyruvate dehydrogenase kinase 4. J Clin Endocrinol Metab, 2007. 92(1): p. 284-92.

24. Brons, C., et al., Impact of short-term high-fat feeding on glucose and insulin metabolism in young healthy men. J Physiol, 2009. 587(Pt 10): p. 2387-97.

25. van Herpen, N.A., et al., Three weeks on a high-fat diet increases intrahepatic lipid accumulation and decreases metabolic flexibility in healthy overweight men. J Clin Endocrinol Metab, 2011. 96(4): p. E691-5.

26. Amati, F., et al., Skeletal muscle triglycerides, diacylglycerols, and ceramides in insulin resistance: another paradox in endurance-trained athletes? Diabetes, 2011. 60(10): p. 2588-97.

27. Bosma, M., et al., Overexpression of PLIN5 in skeletal muscle promotes oxidative gene expression and intramyocellular lipid content without compromising insulin sensitivity. Biochim Biophys Acta, 2013. 1831(4): p. 844-52.

28. Gemmink, A., et al., Decoration of intramyocellular lipid droplets with PLIN5 modulates fastinginduced insulin resistance and lipotoxicity in humans. Diabetologia, 2016. 59(5): p. 1040-8.

29. Haemmerle, G., et al., ATGL-mediated fat catabolism regulates cardiac mitochondrial function via PPAR-alpha and PGC-1. Nat Med, 2011. 17(9): p. 1076-85.

30. Ong, K.T., et al., Adipose triglyceride lipase is a major hepatic lipase that regulates triacylglycerol turnover and fatty acid signaling and partitioning. Hepatology, 2011. 53(1): p. 116-26.

31. Young, S.G. and R. Zechner, Biochemistry and pathophysiology of intravascular and intracellular lipolysis. Genes Dev, 2013. 27(5): p. 459-84. 
32. Pollak, N.M., et al., Cardiac-specific overexpression of perilipin 5 provokes severe cardiac steatosis via the formation of a lipolytic barrier. J Lipid Res, 2013. 54(4): p. 1092-102.

33. Wolins, N.E., et al., OXPAT/PAT-1 is a PPAR-induced lipid droplet protein that promotes fatty acid utilization. Diabetes, 2006. 55(12): p. 3418-28.

34. Sanders, M.A., et al., Endogenous and Synthetic ABHD5 Ligands Regulate ABHD5-Perilipin Interactions and Lipolysis in Fat and Muscle. Cell Metab, 2015. 22(5): p. 851-60.

35. Bosma, M., et al., The lipid droplet coat protein perilipin 5 also localizes to muscle mitochondria. Histochem Cell Biol, 2012. 137(2): p. 205-16.

36. Russell, A.P., et al., Lipid peroxidation in skeletal muscle of obese as compared to endurance-trained humans: a case of good vs. bad lipids? FEBS Lett, 2003. 551(1-3): p. 104-6.

37. Demozay, D., et al., FALDH reverses the deleterious action of oxidative stress induced by lipid peroxidation product 4-hydroxynonenal on insulin signaling in 3T3-L1 adipocytes. Diabetes, 2008. 57(5): p. 1216-26.

38. Ingram, K.H., et al., Skeletal muscle lipid peroxidation and insulin resistance in humans. J Clin Endocrinol Metab, 2012. 97(7): p. E1182-6.

39. Bergstrom, J., Percutaneous needle biopsy of skeletal muscle in physiological and clinical research. Scand J Clin Lab Invest, 1975. 35(7): p. 609-16.

40. Schwartz, D.M. and N.E. Wolins, $A$ simple and rapid method to assay triacylglycerol in cells and tissues. J Lipid Res, 2007. 48(11): p. 2514-20.

41. van de Weijer, T., et al., Effects of bezafibrate treatment in a patient and a carrier with mutations in the PNPLA2 gene, causing neutral lipid storage disease with myopathy. Circ Res, 2013. 112(5): p. e514. 


\section{Supplemental Figures}

A

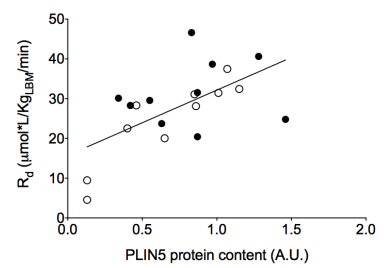

C

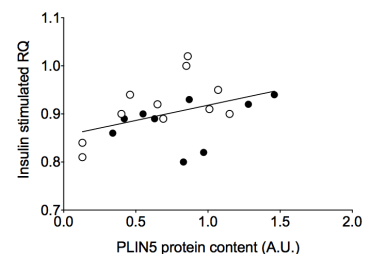

E
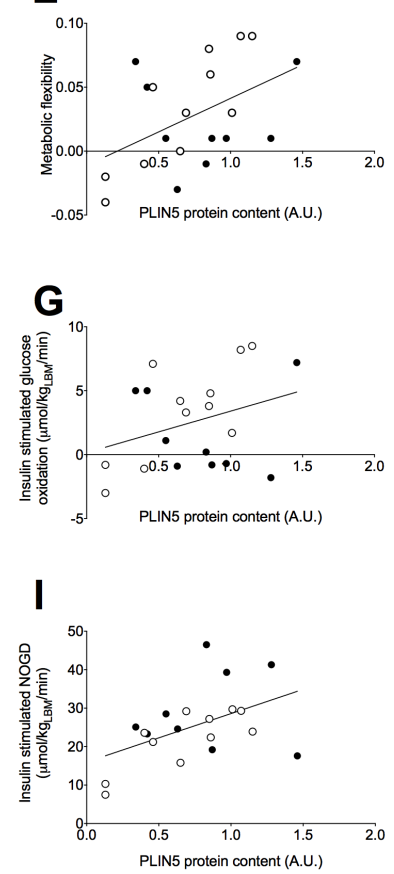

B

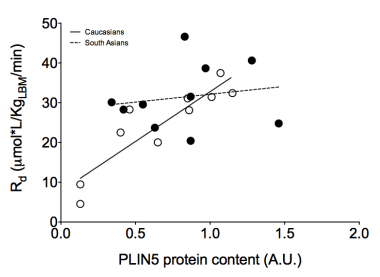

D

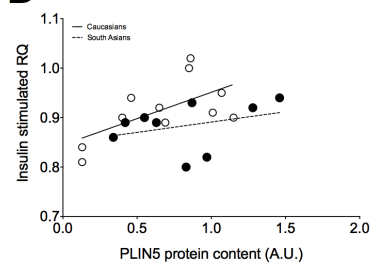

$\mathbf{F}$
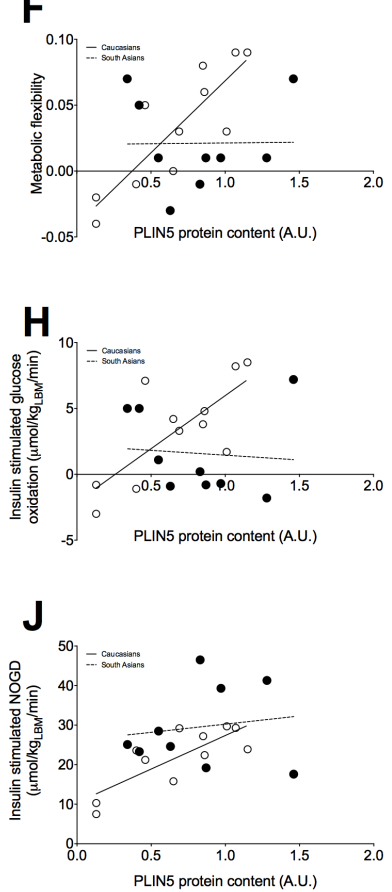

Supplementary Figure S1. Significant correlations from Table 1 between PLIN5 and insulin sensitivity markers before the diet. (A, B) PLIN5 vs. glucose disposal. (C, D) PLIN5 vs. insulin stimulated RQ. (E, F) PLIN5 vs. metabolic flexibility. $(G, H)$ PLIN5 vs. insulin stimulated glucose oxidation. (I, J) PLIN5 vs. insulin stimulated NOGD; Caucasians: o, South Asians: • 
A

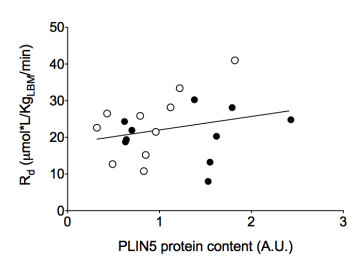

C

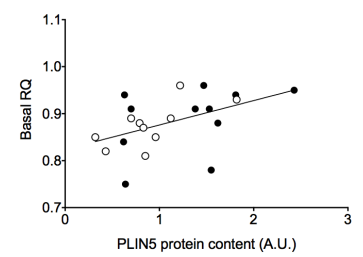

E

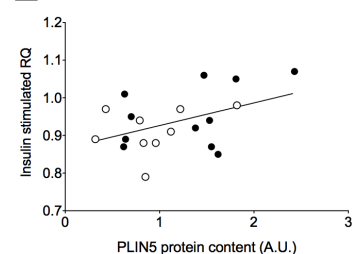

G

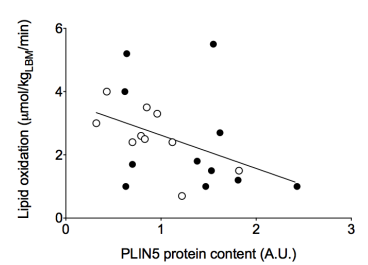

I

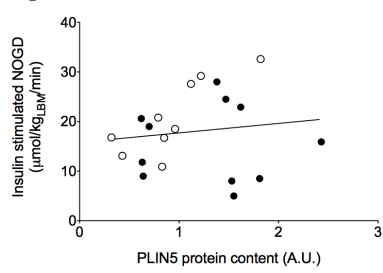

B

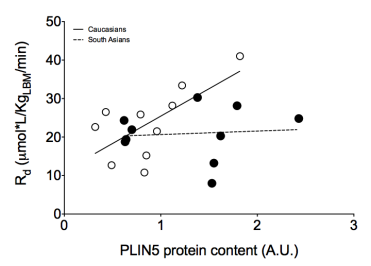

D

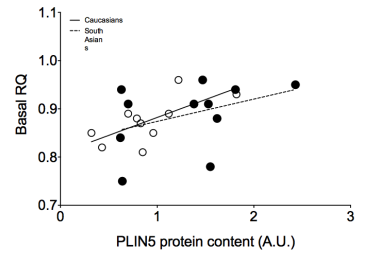

$\mathbf{F}$

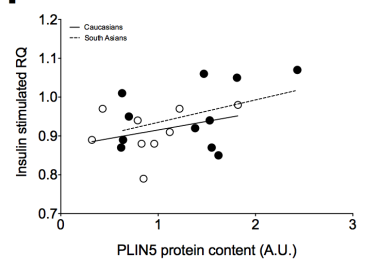

H

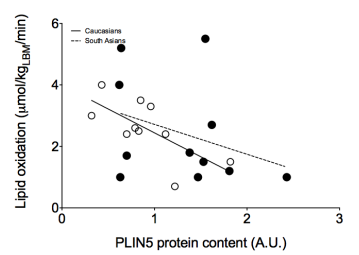

$\mathbf{J}$

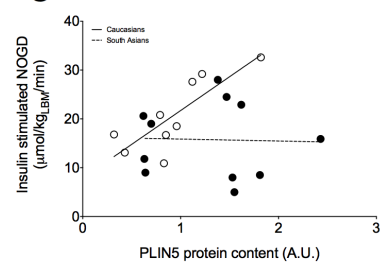

Supplementary Figure S2. Significant correlations from Table 1 between PLIN5 and insulin sensitivity markers after the diet. (A, B) PLIN5 vs. glucose disposal. (C, D) PLIN5 vs. basal RQ. (E, F) PLIN5 vs. insulin stimulated RQ. (G, H) PLIN5 vs. lipid oxidation. (I, J) PLIN5 vs. insulin stimulated NOGD; Caucasians: 0 , South Asians: 


\section{CHAPTER 3}

\section{A microscopy journey to examine lipid droplets, Perilipin 5, and mitochondria in muscle}

Anne Gemmink, Sabine Daemen, Helma J. Kuijpers, Gert Schaart, Patrick Schrauwen, Marc A.M.J. van Zandvoort, Matthijs K.C. Hesselink 


\section{ABSTRACT}

Classical approaches to study intramyocellular lipid (IMCL) content rely on triglyceride extractions. Albeit valuable from a quantitative and qualitative viewpoint, this method does not permit measurements of lipid droplet size, number and location; important factors in the athlete's paradox. Oil-red-O is a commonly used lipid soluble dye to visualize intracellular lipid droplets. Nowadays, these lipid droplets are seen as dynamic organelles decorated with a large variety of proteins. Disadvantages of Oil-red-O are its broad emission spectrum and at least in cultured cells its staining procedure causes fusion of LDs which makes it impossible to reliable image lipid droplet morphology. For the research outlined in this thesis, we aimed to visualize with a 3D confocal approach how PLIN5 coats LDs. PLIN5 is involved in regulating oxidative lipolysis. In line with this we have reported the presence of PLIN5 in near vicinity of mitochondria, as well as associated with the inner mitochondrial membrane. To further explore the precise location of PLIN5 on lipid droplets, the interaction of PLIN5 on the lipid droplet with mitochondria, and the presence of PLIN5 within the mitochondria we set-up a super-resolution immunofluorescent approach by taking advantage of STimulation Emission Depletion (STED) microscopy.

Here we describe the set-up needed for 3D imaging of PLIN5 in skeletal muscle biopsies. Subsequently, we discuss the choice of fluorophores for appropriate single and dual color STED imaging in skeletal muscle tissue and human primary myotubes. We used STED microscopy to discriminate between the inner and outer mitochondrial membranes to further examine PLIN5 presence on/in the mitochondria.

While fluorescence widefield microscopy with a 100 times magnification shows that PLIN5 is surrounding the lipid droplet, 2D and 3D confocal immunofluorescence microscopy shows that PLIN5 is present at the lipid droplets as intensified spots. We successfully set-up dual color STED imaging whereby we can distinguish the inner and outer membrane in human primary myotubes. Subsequently, we applied this dual color STED imaging in human skeletal muscle sections. We show that in human skeletal muscle sections PLIN5 is present in close proximity of the mitochondria. However, STED imaging of the inner mitochondrial membrane in skeletal muscle sections should be further optimized to draw definitive conclusions about PLIN5 localization in the mitochondria. 


\section{INTRODUCTION}

Intramyocellular lipid (IMCL) levels in skeletal muscle is negatively associated with insulin sensitivity. However, endurance trained athletes have similar as or even higher levels of IMCL than type 2 diabetes patients whilst being very insulin sensitive [1]. Upon a combined diet and training program obese subjects who decreased most in lipid droplet (LD) size, increased most in insulin sensitivity [2]. Besides LD size, it is also suggested by several observations that LD number [3] and subcellular distribution of LDs [4] somehow contribute to the observed association between IMCL content and insulin sensitivity. In later stages, sequestering of bioactive (insulin desensitizing) lipids and the proteins involved in LD turnover have been suggested to affect the association between IMCL content and insulin sensitivity. Classically, approaches to quantify muscle fat content rely on extraction of triglycerides from the muscle. Thus, these approaches cannot provide information on LD size, number, subcellular distribution, coating of the LD with proteins involved in LD dynamics or LD organelle interaction. Most of these phenomena can only be examined using microscopic imaging.

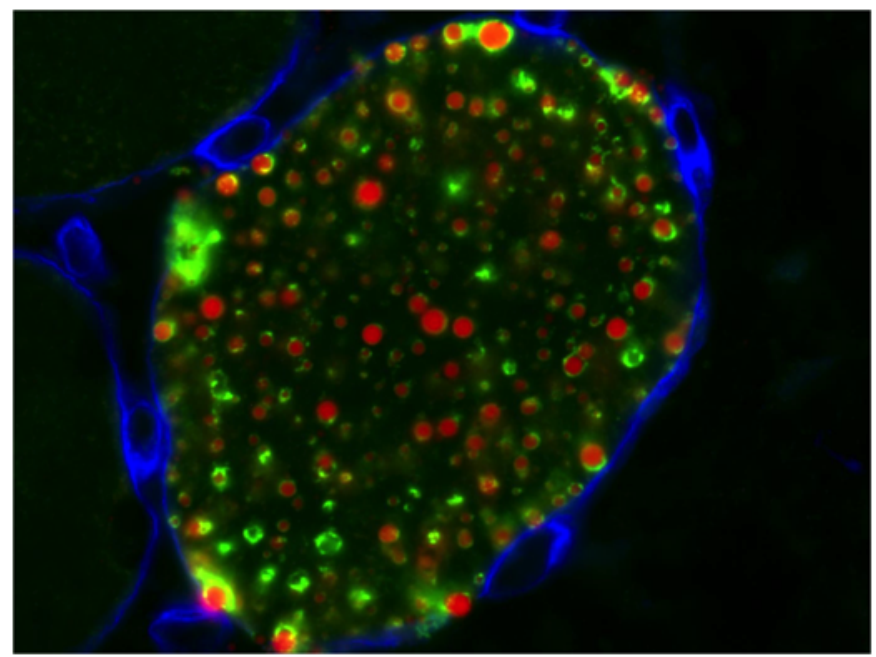

Figure 1. PLIN5 imaged by widefield fluorescence microscopy with 100 times magnification. High magnification widefield fluorescence microscopic image showing that PLIN5 (green) is surrounding the lipid droplets stained by Oil-red-O (red) in total; cell membrane is stained with laminin (blue) in rat m. tibialis anterior overexpressing PLIN5.

Using Oil-red-O as a lipid soluble dye, combined with conventional widefield immunofluorescence microscopy, we and others have been able to semi-quantify LD content in muscle and show fiber type specific differences [5] and decoration of myocellular LDs with PLIN2 and PLIN5 [6]. From these 2-dimensional images, it appeared that LD decorating proteins, like PLIN2 and PLIN5, entirely mantle myocellular lipid droplets. For more detailed analyses of volume of the LD, however, the z-resolution of conventional widefield microscopy is insufficient and the ill-defined fluorescent characteristics of Oil-red-O complicate the distinction between background staining and LD-derived signal. 
Furthermore, it has been reported before that the staining procedure of Oil-red-O induces LD fusion in cultured cells, rendering it a less appropriate dye to study LD morphology [7]. Thus, we aimed to optimize LD detection and size assessment by using dyes with better fluorescent characteristics, like a narrow emission spectrum, and imaging with higher resolution. To examine if the observed decoration of LD with LD coat proteins, which in a 2-dimensional image represents as a rim encompassing the LD (Figure 1), truly reflects the physiological state or may originate from out-offocus signal, and a low resolution, we aimed to develop a 3-dimensional reconstruction of LD by confocal microscopy.
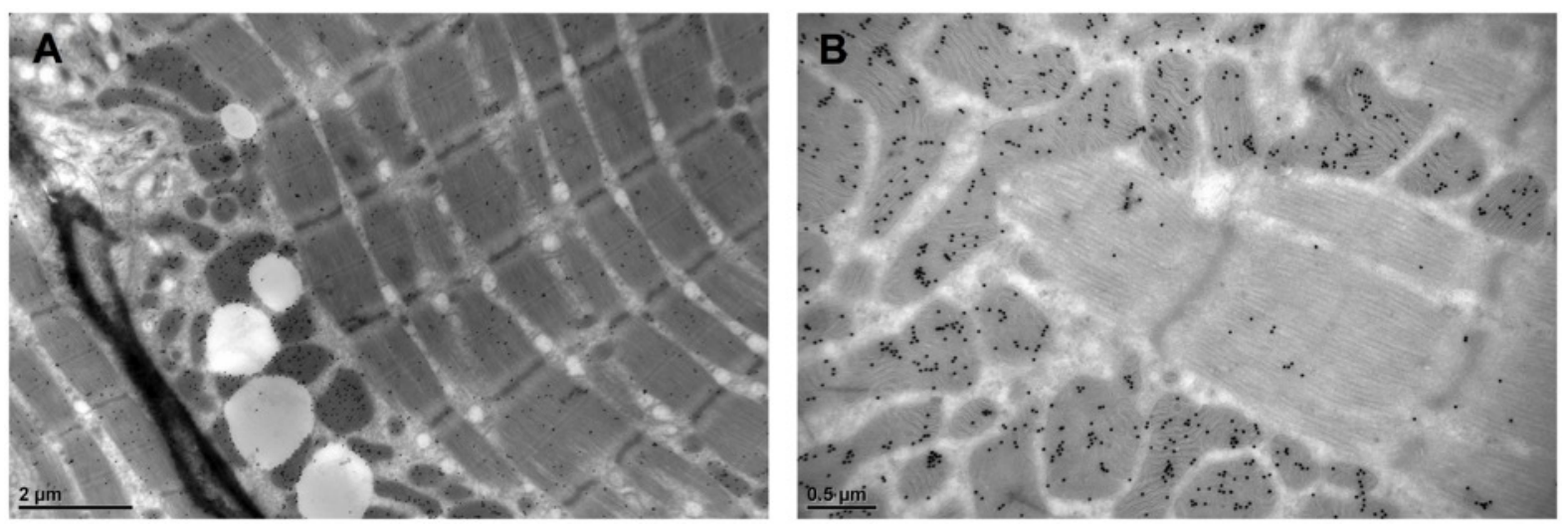

Figure 2. Transmission electron microscopy images showing PLIN5 on LD-mitochondria contact sites and the inner mitochondrial membrane. (A) Immunogold labeled-PLIN5 is present at the LD-mitochondria contact sites. (B) Immunogold shows specific labeling of PLIN5 on the inner mitochondrial membrane and no random labeling in the mitochondrial matrix and outer membrane.

Of the lipid droplet coat proteins known, PLIN5 stands out for its hypothetical role in regulating oxidative lipolysis $[8,9]$. In support of this notion, we have observed a tight correlation between PLIN5 content in muscle homogenates and mitochondrial respiration, an association that in the same samples was absent for PLIN2 [10]. Double immunofluorescence against PLIN5 and mitochondrial marker proteins using widefield fluorescence microscopy with a 100 times magnification suggests co-localization of PLIN5 with mitochondria. These observations were confirmed at a higher resolution by immunogold electron microscopy. Using nano-gold particles, we observed not only prominent staining of PLIN5 at the interface of mitochondria and lipid droplets, but also observed PLIN5-derived signal on mitochondrial cristae as well [10] (Figure 2). We, and others, have suggested that a physical, possibly PLIN5-mediated, link between lipid droplets and mitochondria may serve to shuttle lipid droplet lipolysis-derived fatty-acids towards oxidation [8, 10]. Our combined immunofluorescence and electron microscopy observations, however, leave a 
couple of concerns unresolved. First, while the widefield immunofluorescence images of lipid droplets and PLIN5 seem to suggest that the entire lipid droplet is surrounded by PLIN5, the immunogold electron microscopy suggests localization of PLIN5 at the interface of lipid droplets and mitochondria solely. Second, both high magnification widefield fluorescence microscopy and electron microscopy reveal that PLIN5 can also be found within mitochondria and more specifically, in association with the inner mitochondrial membrane or cristae [10]. Most commonly (although not exclusively) inner mitochondrial membrane proteins are characterized by a mitochondrial targeting peptide which delivers the pre-protein to the mitochondrion for incorporation. For PLIN5, no such targeting peptide has been described. Lastly, we recently reported that upon fasting, PLIN5 seems to relocate from unknown, and hence not yet characterized, cytosolic pools towards specific loci on the lipid droplet [3], whereas others have reported motility of lipid droplets along with promoted interorganelle interaction [11].
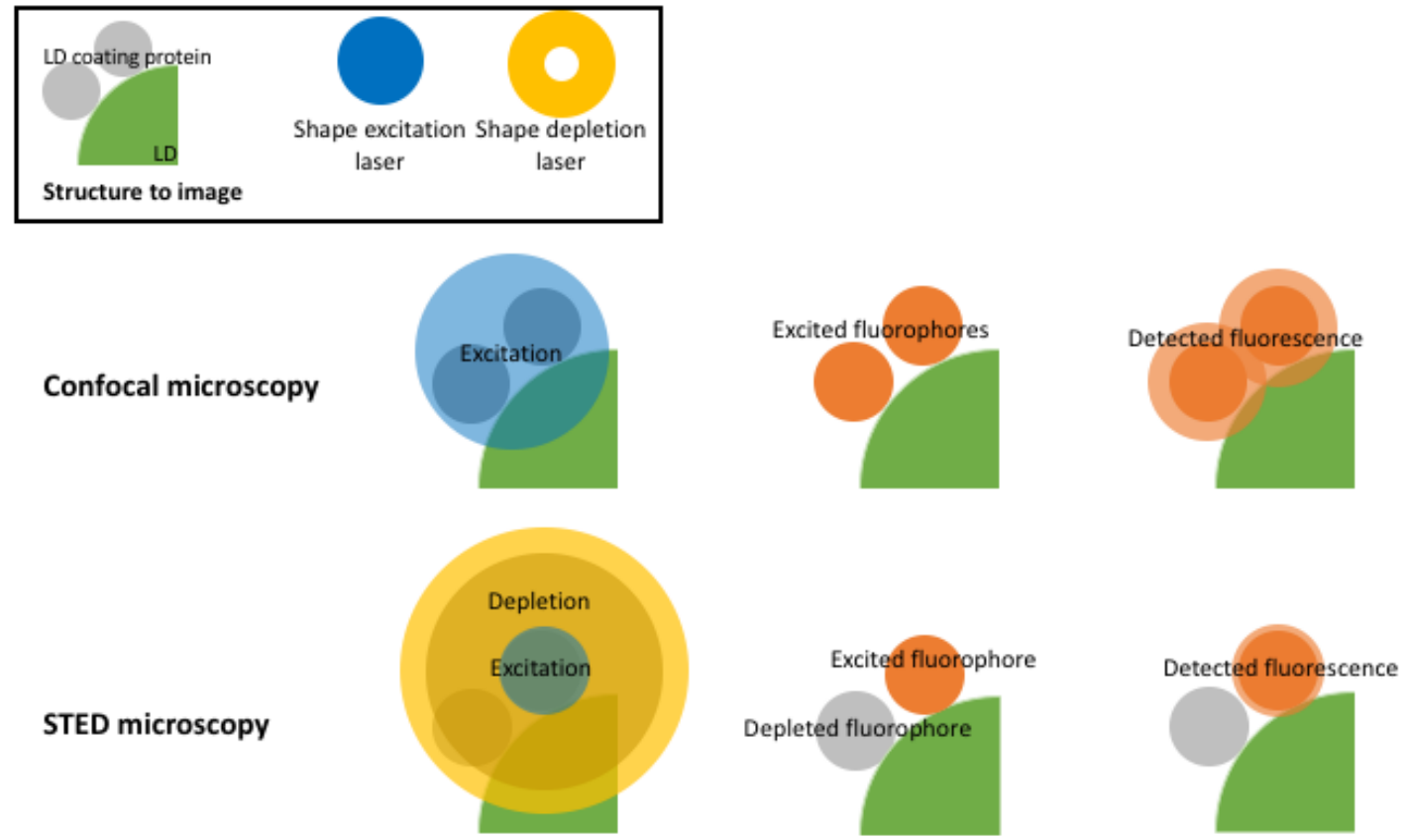

Figure 3. Confocal fluorescence microscopy versus STED microscopy. In the upper left corner, the structure (a lipid droplet coating protein of interest) to be imaged is depicted. STED microscopy has two lasers, an excitation laser, and a depletion laser. The depletion laser is doughnut-shaped. When one point is excited with confocal fluorescence microscopy two particles on the lipid droplet are excited. Both particles will emit light and will be detected as one oval-shaped particle. When using STED microscopy, the excitation and depletion laser are used at the same time. The excitation laser will excite both particles, and in this case the depletion laser will deplete one particle. As a result, fluorescence of one particle is detected. 
To examine the conditions affecting PLIN5 and/or lipid droplet motility, PLIN5-mediated lipid droplet-mitochondria interaction, and the putative role of PLIN5 within mitochondria, higher imaging resolution than confocal fluorescence microscopy is warranted. In confocal fluorescence microscopy, the resolution is improved in comparison with widefield fluorescence microscopy by scanning the sample point-by-point with a laser instead of illuminating the whole sample like with widefield fluorescence microscopy. In addition, the pinhole blocks out-of-focus light and this improves the lateral and axial resolution. Stimulated emission depletion (STED) microscopy is one of the super-resolution microscopy tools in which selective depletion of a single fluorophore can be used to significantly increase the imaging resolution below the diffraction limit of light microscopy [12-14]. STED microscopy improves the resolution further by the use of two lasers. One laser to excite the fluorophore to be imaged and a doughnut-shaped depletion laser that depletes the emitted light at the peripheral resulting in only light emitted at the center focal point (Figure 3). The lateral resolution obtained with confocal microscopy is $200 \mathrm{~nm}$, while commercial STED systems can reach a lateral resolution of $60 \mathrm{~nm}$.

Thus, the final aim of this study is to set-up STED based imaging of PLIN5, lipid droplets and mitochondria to examine the precise location of PLIN5 on the lipid droplet, the interaction of PLIN5 on the lipid droplet with mitochondria and to elucidate putative presence of PLIN5 within mitochondria.

\section{METHODS}

\section{Immunohistochemistry for confocal microscopy}

For 3D reconstruction of LDs with PLIN5, 7 $\mu \mathrm{m}$ thick skeletal muscle sections were cut and fixated for 30 minutes with $3.7 \%$ formaldehyde in PBS. Sections were blocked for 45 minutes with blocking buffer (2\% BSA, $150 \mathrm{mM} \mathrm{NaCl}$ and $20 \mathrm{mM}$ Tris pH 6.8) and subsequently permeabilized for 5 minutes with $0.25 \%$ TX-100. After washing the sections for 5 minutes with PBS, sections were incubated with primary antibodies against PLIN5 (GP31, Progen Biotechnik, Heidelberg, Germany) and Laminin (L9393, Sigma, MO, USA) diluted 1:25 in 0.05\% Tween/PBS. Subsequently, sections were incubated with Bodipy 493/503 (D3922, Molecular Probes, Leiden, The Netherlands) diluted 1:100 and the appropriate conjugated secondary antibodies Alexa Fluor 405 and Alexa Fluor 647, for respectively laminin and PLIN5 in $0.05 \%$ Tween/PBS. Sections were mounted with \#1 coverslips in Mowiol and stored in the dark until imaging. 


\section{Confocal fluorescence microscopy and 3D reconstruction of LDs}

Sections were imaged on a Leica TCS SPE confocal microscope using a $63 \times 1.3$ N.A. oil immersion objective with a 1.1 optical zoom at $2048 \times 2048$ pixels, resulting in a pixel size of $77 \mathrm{~nm}$ by $77 \mathrm{~nm}$ with a theoretical resolution of $200 \mathrm{~nm}$ by $200 \mathrm{~nm}$. The 405, 488, and 635 laser lines were used for imaging Laminin-AF405, Bodipy 493/503 and PLIN5-AF647, respectively. Emission was detected between $415-460 \mathrm{~nm}, 500-560 \mathrm{~nm}$, and 650-750, respectively. To ensure proper deconvolution pixel oversaturation was prevented and a z-distance of $0.17 \mu \mathrm{m}$ with a theoretical resolution of $500 \mathrm{~nm}$ was used. For two-dimensional images z-stacks of 4 slices were taken to be able to do proper deconvolution. Huygens Essential (Scientific Volume Imaging B.V., Hilversum, The Netherlands) was used for deconvolution of the acquired images. For creating 3D images of LDs and their PLIN5 decoration complete z-stacks were loaded into the 3D viewer plugin [15] in ImageJ [16].

\section{Immunohistochemistry for STED microscopy}

For optimization of the BD Horizon V500 (V500) fluorophore, $5 \mu \mathrm{m}$ thick sections of ZDF rat $\mathrm{m}$. tibialis anterior were cut and fixated with 3.7\% formaldehyde in PBS. Subsequently, the muscle sections were permeabilized with $0.25 \%$ TX100 for 5 minutes. Muscle sections were incubated with a primary antibody against PLIN5 1:25 for 45 minutes. Subsequently, muscle sections were incubated with Biotin-SP GaGp (106-065-003, BioConnect, Huissen, The Netherlands) 1:400 in 0.05 Tween/PBS for 45 minutes. After the Biotin step, muscle sections were incubated with StreptavidinHorizon V500 (561419, BD Biosciences, Breda, The Netherlands) 1:50 for 45 minutes as well. Sections were mounted with \#1.5 coverslips with Mowiol and stored in the dark until imaging.

Dual color images were performed in primary human myotubes and in $5 \mu \mathrm{m}$ thick human skeletal muscle sections. Human primary myotubes were cultured on coverslips and differentiated for 8 days. Myotubes were fixated for 15 minutes with $3.7 \%$ formaldehyde in PBS and permeabilized for 5 minutes in $0.25 \%$ TX-100 in PBS. Primary antibodies used were TOMM20 (ab8937, Abcam, Cambridge, United Kingdom) and the antibody cocktail against the 5 complexes of the electron transport chain (OXPHOS) (MS601, BioConnect, Huissen, The Netherlands) 1:25 in 0.05\% Tween/PBS. Secondary antibodies for TOMM20 and OXPHOS were Abberior STAR 440 (AS440) (2-0012-003-4, Abberior, Göttingen, Germany) and Alexa Fluor 488 (AF488), respectively. For skeletal muscle the same fixation and permeabilization procedures were used as described for the single PLIN5-V500. Sections were incubated for 45 minutes with primary antibodies against PLIN5 and TOMM20 1:25 or 
PLIN5 and OXPHOS 1:25 in 0.05\% Tween/PBS. Subsequently, sections were incubated for 45 minutes with the appropriate conjugated secondary antibodies AF488 and AS440 for respectively PLIN5 and TOMM20 or OXPHOS. Sections and coverslips were mounted with Mowiol and stored in the dark until imaging.

\section{STED microscopy}

To acquire STED images a Leica TCS SP8 STED microscope was used with a 100x 1.4 N.A. objective. Images consisted of $1024 \times 1024$ pixels and contained a pixel size below $30 \mathrm{~nm}$ by $30 \mathrm{~nm}$. Both the white light laser and the $592 \mathrm{~nm}$ depletion laser are pulsed lasers and needs to be aligned before use to get an optimal STED result. To obtain an optimal separation of AS440 and AF488, emission of AS440 was collected at wavelengths where AF488 has no or limited emission, AF488 was excited at a wavelength which barely excites AS440. AS440 and AF488 were excited with a wavelength of 470 $\mathrm{nm}$ and $518 \mathrm{~nm}$, respectively. Emission was detected between $475 \mathrm{~nm}$ and $500 \mathrm{~nm}$ for AS440, and for AF488 between $545 \mathrm{~nm}$ and $560 \mathrm{~nm}$. For further improvements of the spatial resolution, gating can be used to detect only photons within a range of lifetimes. In addition, a pinhole of 0.9 Airy Unit was used to further improve the resolution by blocking more out-of-focus light. Subsequently, a higher intensity of the STED laser can improve the resolution as well. However, a balance needs to be found between improved resolution and quenching the fluorophore. All images were deconvolved with Huygens Professional (Scientific Volume Imaging B.V., Hilversum, The Netherlands). Images were first checked for oversaturated pixels and subsequently for hot pixels (pixels that are much brighter than they should be). Oversaturated and hot pixels can interfere with the deconvolution process. In case of any hot pixels, those have been removed from the image and the deconvolution process was started. Images were deconvolved with 15 iterations and a signal-tonoise ratio set at 7 .

\section{PLIN5 detection in human primary myotubes}

Human primary myocytes were differentiated into fused myotubes on coverslips and incubated with $400 \mu \mathrm{M}$ oleate to stimulate LD formation. Myotubes were promptly frozen in melting isopentane and subsequently fixated with 3.7\% formaldehyde in PBS for 15 minutes. Similar staining procedures for PLIN5 were followed as described under 'immunohistochemistry for STED microscopy'. In addition, PLIN5 was co-stained with DAPI, to visualize the nuclei, and with the proliferation marker 
Ki67, for visualization of the nucleolus. Furthermore, we also tested different permeabilization agents on primary myotubes grown on coverslips and fixated for 15 minutes with $3.7 \%$ formaldehyde. Instead of using $0.10 \%$ TX100 for 5 minutes we used $0.001 \%$ SDS and $100 \mu$ M DPS.

\section{RESULTS AND DISCUSSION}

\section{Confocal imaging of lipid droplets and PLIN5 in human skeletal muscle sections}

In many studies an Oil-red-O staining is imaged on a conventional widefield fluorescence microscope with a $40 x$ objective to quantify IMCL content in a fiber-type specific fashion. As it has been frequently reported, it is not the magnitude of $I M C L$ that interferes with insulin sensitivity, but rather the way IMCL is stored along with the dynamic nature of the of LDs [17]. Therefore, we aimed to develop more sophisticated ways to quantify these parameters. For a more detailed analysis of IMCL content, like LD size, number, and distribution, a higher resolution is needed. In addition, to accurately measure LD number and putative co-localization between LDs and LD coating proteins, confocal fluorescence microscopy is essential. In cultured cells it was observed that the staining procedure for Oil-red-O fuses LDs, which affects LD morphology consequently [7]. To measure LD decoration a LD soluble dye is needed that has no effect on LD morphology. Such a dye is Bodipy 493/503 [18]. Flow cytometry showed that Bodipy 493/503 is more specific for LDs than is Oil-red-O [19]. The emission spectrum of Bodipy 493/503 is more narrow than Oil-red-O [18]. Bodipy 493/503 has an emission peak around $513 \mathrm{~nm}$. Bodipy 493/503 stains not only the neutral lipids present in LDs, but also the neutral lipids present in membranes. When collecting emission of Bodipy 493/503 between wavelengths of 570 and $650 \mathrm{~nm}$ most of the light comes from lipid droplets.

To study LD decoration by PLIN5 in more detail we used Bodipy $493 / 503$ to stain LDs in skeletal muscle samples along with the basement membrane maker laminin. Deconvolution was performed on images acquired on a confocal fluorescence microscope. To perform high quality quantifications on the acquired images, deconvolution is an important step before the actual analyses of LD size. As shown in Figure 4, deconvolution removes noise introduced by the system, improves signal-to-noise ratio, and moreover it corrects for microscopic aberration introduced by i.e. the objectives. Due to deconvolution the rims of LDs become better defined and LD size can be measured more accurately. Next, we made 3D reconstructions of LDs from z-stacks obtained on a confocal microscope to explore if PLIN5 decorates the LD by forming a ring-shaped structure around the LD or if PLIN5 localizes as distinct spots on the LD. Figure 5 reveals that PLIN5 does not appear in a ring around the LD, but rather is apparent as a single spot to several spots per LD. We have observed these 
intensified PLIN5 spots before on the LD-mitochondria interface [10]. Furthermore, immunogold labeled EM images showed that PLIN5 was also present in the mitochondria, even on the mitochondrial cristae (Figure 2).
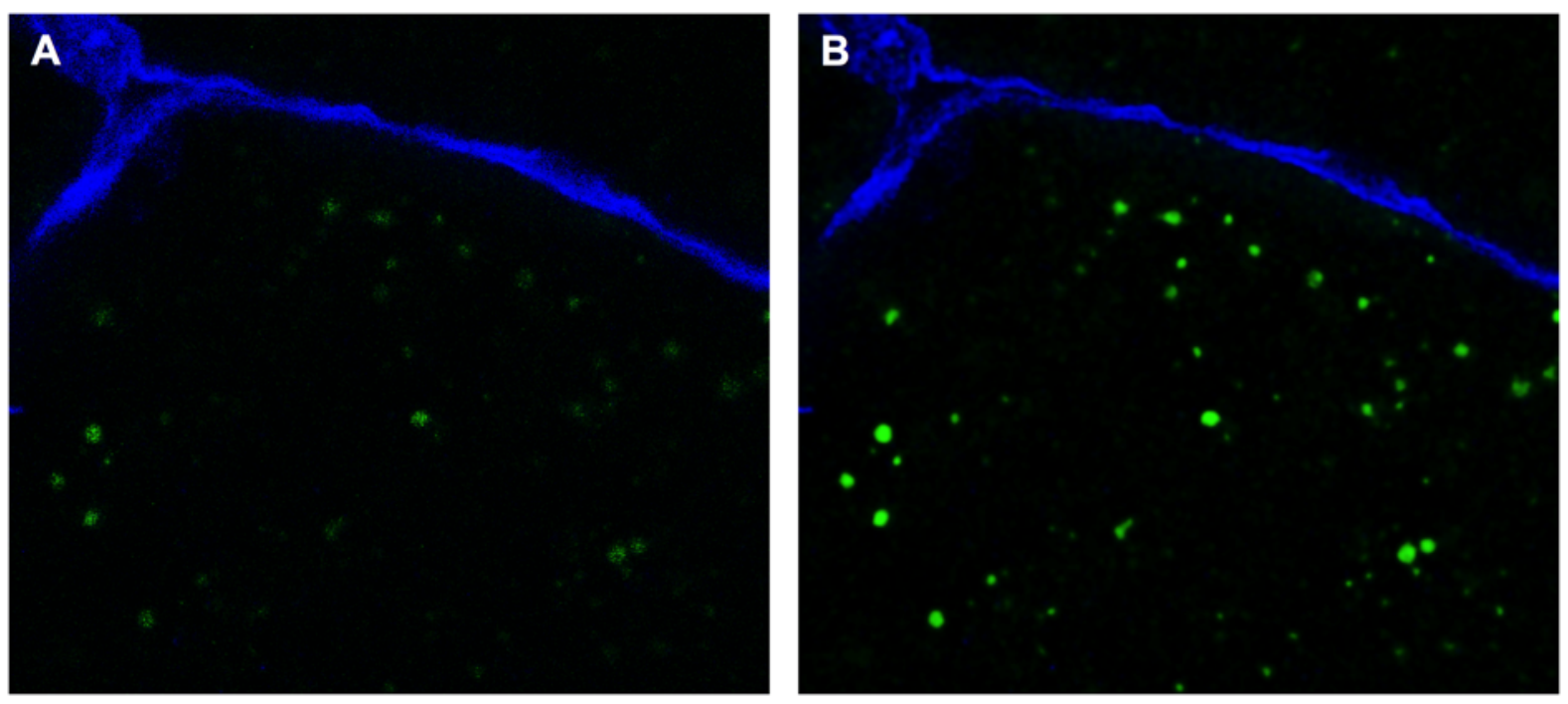

Figure 4. Deconvolution improves signal-to-noise ratio and sharpens edges of objects. (A) Raw confocal image. (B) Deconvolved confocal image.

\section{Considerations for STED microscopy}

Next we aimed to confirm the finding that PLIN5 is present in the mitochondria with another imaging approach than Tokuyasu-based or Aurion-based immunogold transmission electron microscopy. The resolving power of conventional confocal fluorescence microscopy is insufficient to explore if PLIN5 is located only at the LD-mitochondrial interface or as well on the inner mitochondrial membrane. The limitations in spatial resolution can nowadays be overcome by taking advantage of so-called super-resolution microscopy, e.g., STED microscopy. With STED microscopy, the resolving power theoretically suffices to distinguish the inner mitochondrial membrane from the outer mitochondrial membrane. With the optimal settings and use of the right probes, a $x y-$ resolution of $60 \mathrm{~nm}$ can be reach with commercial STED systems [13, 20, 21]. To reach this high resolution, several considerations need to be taken into account when selecting probes for STED microscopy. First, to obtain improvements in the resolution the probe used should emit some light at the wavelength of the depletion laser. Furthermore, it is important to use probes that do not absorb at the wavelength of the depletion laser. This is not only important for the probe used for STED microscopy, but also for other probes used for imaging in the confocal mode. Since the depletion laser has much more power, excitation of the probe by this laser will result in bleaching of the probe used. In addition, if a probe is excited by the depletion laser, an anti-stoke shift (emitting 

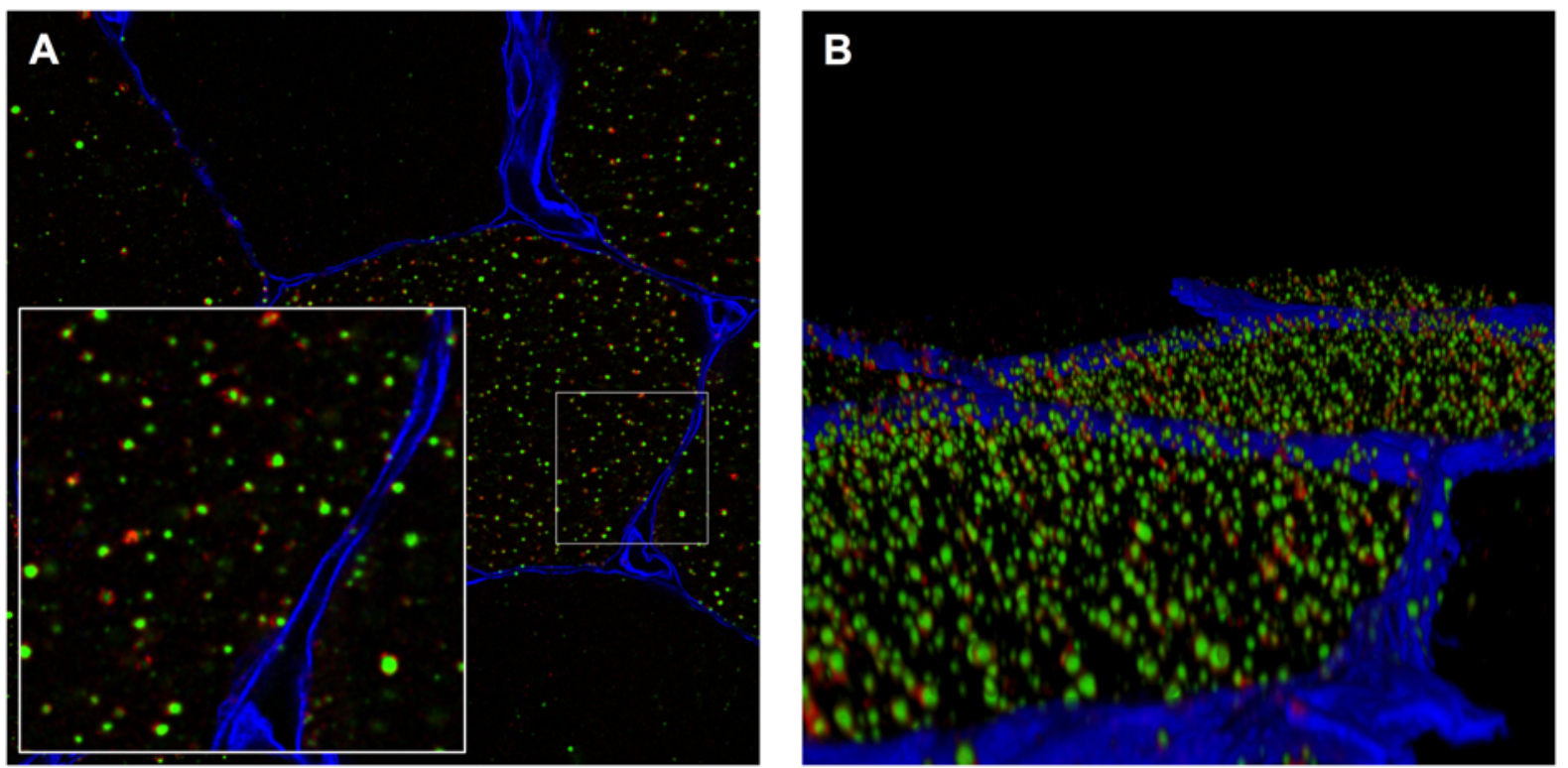

Figure 5. 2D and 3D confocal images of lipid droplets and PLIN5 in skeletal muscle. (A) 2D and (B) 3D confocal images of LDs stained by Bodipy 493/503 (green) and PLIN5 (red) show that PLIN5 is present at the lipid droplets as intensified spots instead of surrounding the lipid droplet in total in human skeletal muscle biopsies from the $\mathrm{m}$. vastus lateralis. Insert in A shows these intensified PLIN5 spot on the LD in a higher magnification.

light at a lower wavelength than the wavelength used for excitation) can occur. Whether or not a probe is excited by the depletion laser can be easily tested by staining only the probe of interest and only switch on the depletion laser and collect emission at the wavelengths used during imaging. Recently, the new and rapidly advancing field of super resolution microscopy welcomed new fluorescent probes which have a long stoke shift and can thus be used to acquire 2-color images. These novel probes can be combined with a probe with a normal stoke shift, like the well-known Alexa Fluors. By combining these two types of probes this results in the use of two fluorophores with a distinct excitation wavelength with an overlapping emission spectra with a weak emission at the wavelength of the depletion laser for STED microscopy. This contrasts with confocal microscopy where fluorophores are chosen with a different excitation and emission spectra for a good separation. Probes with a long stoke shift we have tested are Abberior STAR 440 and BD Horizon V500. As shown in Figure 6A and 6B, the BD Horizon V500 resulted in an improved resolution. Similar results were obtained for the Abberior STAR 440 (data not shown). A second consideration is that the spatial resolution is reduced if the complex of antibodies attached to the protein of interest is larger than the resolving power. Thus, we preferred to use Abberior STAR 440 over the use of BD Horizon V500. The use of the BD Horizon V500 leads to a larger protein-antibody complex, because the secondary antibody is conjugated with biotin and needs to form a complex with streptavidin for the fluorescent label. The final consideration to take into account is that the antibodies need to be stable, since high laser power will be used on the sample. This high laser power from the depletion 
laser leads to bleaching of the sample and needs to be minimized if a z-stack is created. Based on all these considerations we selected Abberior STAR 440 and Alexa Fluor 488 for dual color images.

\section{STED imaging of the inner and outer membrane in human primary myotubes}

To study the presence of PLIN5 in or on the mitochondria we first needed to test if we can distinguish the inner and outer mitochondrial membrane. We tested if we can distinguish the inner and outer mitochondrial membrane in cultured human primary myotubes. For imaging the inner and
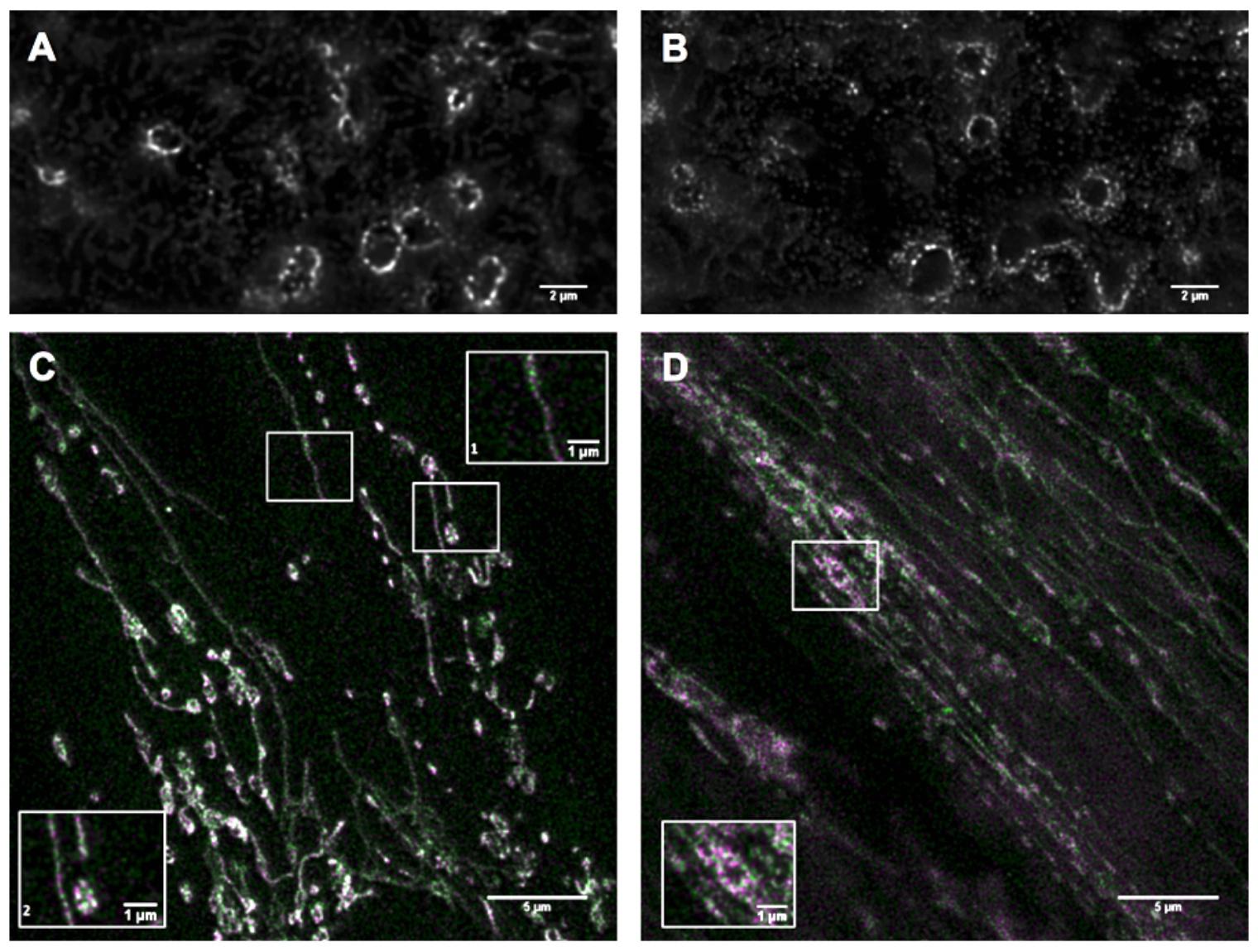

Figure 6. Single and dual color STED imaging. A confocal image (A) versus a STED image (B) of PLIN5 with Horizon V500 as fluorophore shows resolution improvements in $\mathrm{m}$. tibialis anterior of a 12 week old ZDF rat. (C) Dual color image of OXPHOS for the inner mitochondrial membrane (green) and TOMM20 for the outer mitochondrial membrane (magenta) imaged at normal intensity of human primary myotubes. Inserts show that the inner and outer mitochondrial membrane can be separated. (D) Dual color image of OXPHOS (green) and TOMM20 (magenta) of human primary myotubes imaged at lower intensities to separate the inner and outer mitochondrial membrane when mitochondrial abundance is higher (see insert). 
the outer mitochondrial membrane with STED microscopy we used antibodies against TOMM20 and OXPHOS complexes respectively. Figure $6 \mathrm{C}$ and 6D shows a two-color STED image of TOMM20 and OXPHOS. When imaging regions where mitochondrial density is low, TOMM20 and OXPHOS do not have much overlap (Figure 6C). To overcome the oversaturation, we imaged TOMM20 and OXPHOS at lower intensities. Figure 6D shows an example imaged at lower laser intensities. Also in areas with a high mitochondrial density inner and outer mitochondrial membrane can be separated when imaged at lower laser intensities.

\section{STED imaging of PLIN5 and mitochondria in human skeletal muscle sections}

We showed that we were able to distinguish the inner and outer mitochondrial membrane by using STED microscopy. Next we aimed to combine a PLIN5 staining with either TOMM20 or OXPHOS. To test if PLIN5 is colocalized with mitochondria as we have observed previously on electron microscopy images [10], we imaged sections from skeletal muscle for PLIN5 and TOMM20 or PLIN5
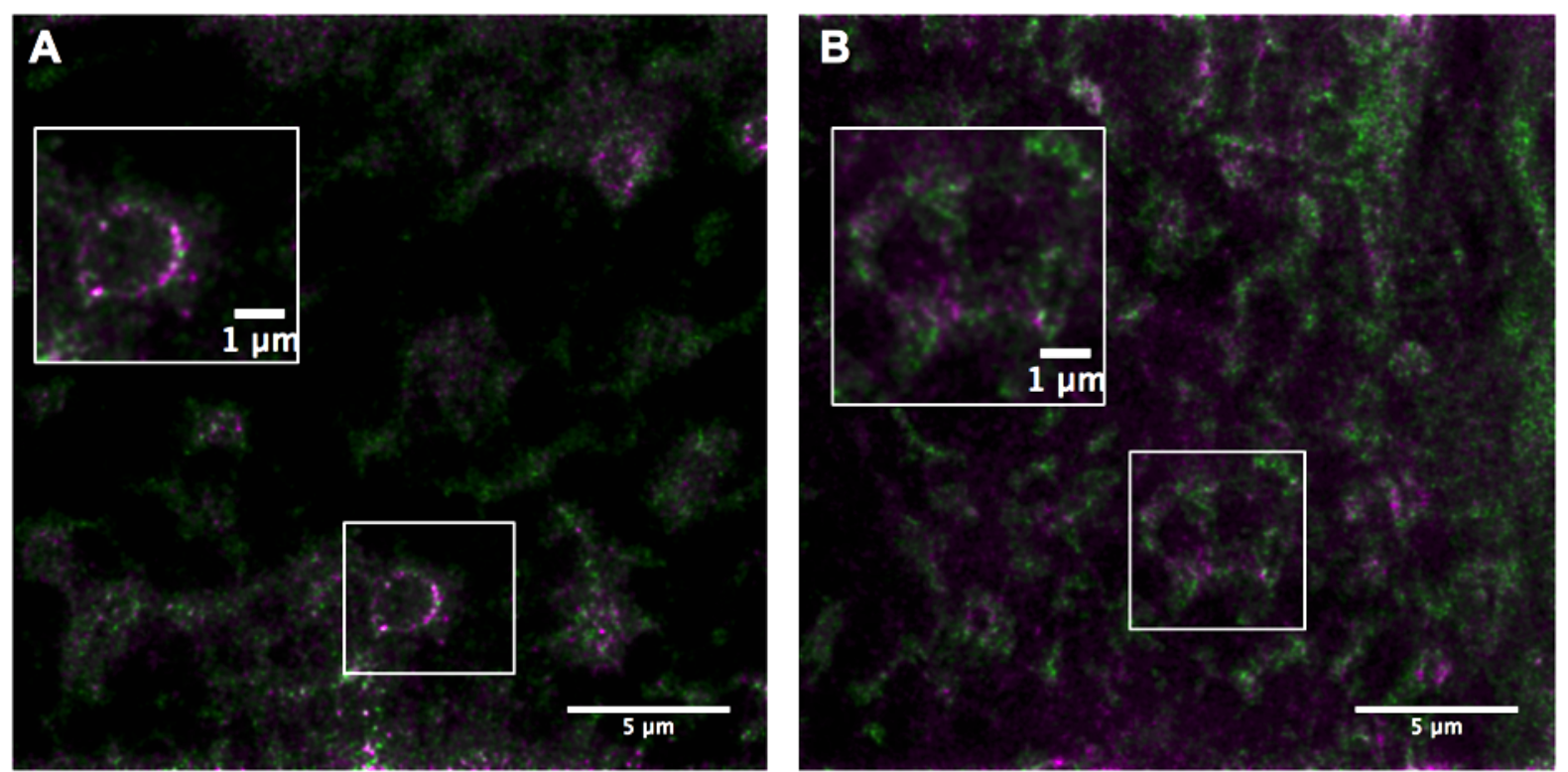

Figure 7. Dual color STED imaging in human skeletal muscle biopsies from the $m$. vastus lateralis. (A) Two-color STED image of PLIN5 (magenta) and the outer mitochondrial membrane labeled by TOMM20 (green). The insert shows that PLIN5 is in close proximity of the outer mitochondrial membrane. (B) STED imaging of PLIN5 (magenta) and the inner mitochondrial membrane labeled by OXPHOS (green). The insert shows that PLIN5 and the inner mitochondrial membrane are in close vicinity. The gain in resolution with STED for OXPHOS is not as much as for TOMM20.

and OXPHOS. In Figure 7A, a representative image is shown and demonstrates that PLIN5 is present in the close proximity of the outer mitochondrial protein TOMM20. Figure 7B shows an image of PLIN5 and OXPHOS, indicating that PLIN5 is not present at the inner membrane, but in close 
proximity. However, STED microscopy resulted in a sharper image for TOMM20 than OXPHOS. Putatively, the staining density of the inner mitochondrial membrane by targeting all five electron transport chain complexes is too dense for skeletal muscle tissue to gain the same amount of resolution when targeting one protein.

\section{PLIN5 imaging in primary myotubes}

Primary myotubes are a relatively easy model to examine conditions in which the interaction between PLIN5, mitochondria, and lipid droplets is affected. However, in contrast to skeletal muscle tissue, in human primary myotubes PLIN5 was detected at different locations than expected. We predominantly observed PLIN5 in a cytosolic fraction and in the nucleus (Figure 8A), rather than on the rims of lipid droplets. The nuclear PLIN5 staining seemed to be specific for the nucleolus. To test
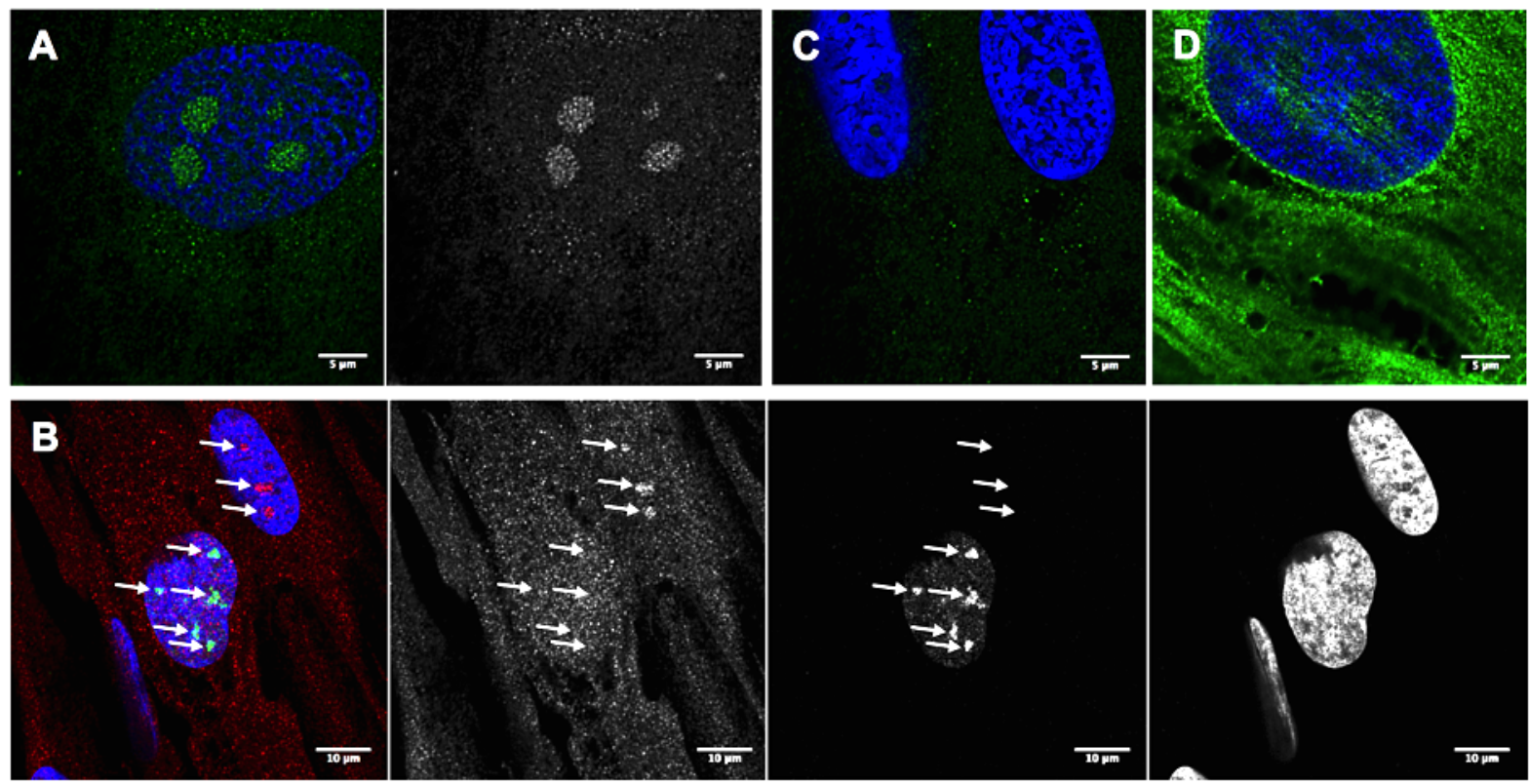

Figure 8. PLIN5 cellular location in primary myotubes is different than in skeletal muscle tissue. (A) In cultured human primary myotubes PLIN5 (green) is present in a cytosolic fraction and the nucleus (blue). In the nucleus PLIN5 is present in dense spots (right panel) in what seems like the nucleolus. (B) Testing if PLIN5 (red) is present in the nucleolus, myotubes were co-stained with DAPI and Ki-67 (green). Arrows are indicating the nucleolus. Middle left (PLIN5), middle right (Ki67) and right (nuclei) panels are the three separated channels. PLIN5 staining intensity is higher in Ki67-negative nucleolus, suggesting presence of PLIN5 in non-proliferating myotubes. ( $C$ and $D$ ) testing if permeabilization with $0.001 \%$ SDS (C) and $100 \mu \mathrm{M}$ DPS (D) affected the staining pattern of PLIN5 in primary myotubes. Permeabilization with $0.001 \%$ SDS showed a somewhat similar pattern as in A, but with less intense staining in the nucleolus, while Permeabilization with $100 \mu \mathrm{M}$ DPS showed a diffuse PLIN5 staining throughout the myotube. 
the specificity of this staining in the nucleolus we co-stained PLIN5 with Ki67, a protein involved in cell proliferation which is present in the nucleolus when cells are proliferating. Co-staining PLIN5 with Ki67 revealed that PLIN5 was present in the nucleolus of non-proliferating human primary myotubes (Figure 8B, see arrows). While we originally considered the nuclear labeling with PLIN5 as an antibody artifact, we recently learned that similar observations have been made by others, showing PLIN5 enrichment in the nucleus upon fasting and during stimulated lipolysis in cultured C2C12 myotubes (personal communication Perry Bickel, FASEB 2016 SRC on Lipid Droplets). To further explore whether the PLIN5 staining pattern in myotubes was an artifact and due to methodological issues, we permeabilized human primary myotubes in multiple ways before applying the first antibody. Permeabilization with $0.001 \%$ SDS showed an almost similar pattern as observed in Figure 8A, but a somewhat less intense staining in the nucleolus (see Figure $8 \mathrm{C}$ ). Permeabilization with $100 \mu \mathrm{M}$ DPS gave a more diffused staining which appeared less specific (Figure 8D). Follow-up studies using antibodies directed against different amino acid sequences of the same protein and on cells under and overexpressing PLIN5 are required to obtain definitive answers, though.

\section{CONCLUSION}

For studying LDs in sufficient detail to make valid statements on how myocellular lipid droplets may be involved in impeding insulin sensitivity, higher spatial imaging resolutions are needed and out-offocus light needs to be blocked. Confocal microscopy is sufficient for examining LD size, number of LDs and to some extend LD decoration. However, for more detailed analysis of LD decoration and the potential interaction between LDs and mitochondria a higher resolution than confocal microscopy is needed. We have shown that, if applied appropriately, STED microscopy provides the resolving power to distinguish the inner and outer mitochondrial membrane. Using STED microscopy, we have not yet been able to confirm previous observations in immunogold transmission electron microscopy that PLIN5 is associated with the outer and inner mitochondrial membrane. Rather, our STED results indicate that PLIN5 is in close proximity of the mitochondria. However, STED imaging of the inner mitochondrial membrane in skeletal muscle should be further optimized by choosing an antibody against one protein, rather than the antibody cocktail we used now, which detects structural proteins in any of the 5 complexes of the inner mitochondrial membrane electron transport chain. 


\section{REFERENCES}

1. Goodpaster, B.H., et al., Skeletal muscle lipid content and insulin resistance: evidence for a paradox in endurance-trained athletes. J Clin Endocrinol Metab, 2001. 86(12): p. 5755-61.

2. He, J., B.H. Goodpaster, and D.E. Kelley, Effects of weight loss and physical activity on muscle lipid content and droplet size. Obes Res, 2004. 12(5): p. 761-9.

3. Gemmink, A., et al., Decoration of intramyocellular lipid droplets with PLIN5 modulates fastinginduced insulin resistance and lipotoxicity in humans. Diabetologia, 2016. 59(5): p. 1040-8.

4. Nielsen, J., et al., Increased subsarcolemmal lipids in type 2 diabetes: effect of training on localization of lipids, mitochondria, and glycogen in sedentary human skeletal muscle. Am J Physiol Endocrinol Metab, 2010. 298(3): p. E706-13.

5. Koopman, R., G. Schaart, and M.K. Hesselink, Optimisation of oil red $O$ staining permits combination with immunofluorescence and automated quantification of lipids. Histochem Cell Biol, 2001. 116(1): p. 63-8.

6. Minnaard, R., et al., Adipocyte differentiation-related protein and OXPAT in rat and human skeletal muscle: involvement in lipid accumulation and type 2 diabetes mellitus. J Clin Endocrinol Metab, 2009. 94(10): p. 4077-85.

7. Fukumoto, S. and T. Fujimoto, Deformation of lipid droplets in fixed samples. Histochem Cell Biol, 2002. 118(5): p. 423-8.

8. Wang, H., et al., Perilipin 5, a lipid droplet-associated protein, provides physical and metabolic linkage to mitochondria. J Lipid Res, 2011. 52(12): p. 2159-68.

9. Sanders, M.A., et al., Endogenous and Synthetic ABHD5 Ligands Regulate ABHD5-Perilipin Interactions and Lipolysis in Fat and Muscle. Cell Metab, 2015. 22(5): p. 851-60.

10. Bosma, M., et al., The lipid droplet coat protein perilipin 5 also localizes to muscle mitochondria. Histochem Cell Biol, 2012. 137(2): p. 205-16.

11. Murphy, S., S. Martin, and R.G. Parton, Lipid droplet-organelle interactions; sharing the fats. Biochimica et Biophysica Acta (BBA) - Molecular and Cell Biology of Lipids, 2009. 1791(6): p. 441-447.

12. Klar, T.A., et al., Fluorescence microscopy with diffraction resolution barrier broken by stimulated emission. Proc Natl Acad Sci U S A, 2000. 97(15): p. 8206-10.

13. Hell, S.W. and J. Wichmann, Breaking the diffraction resolution limit by stimulated emission: stimulated-emission-depletion fluorescence microscopy. Opt Lett, 1994. 19(11): p. 780-2.

14. Klar, T.A. and S.W. Hell, Subdiffraction resolution in far-field fluorescence microscopy. Opt Lett, 1999. 24(14): p. 954-6.

15. Schmid, B., et al., A high-level 3D visualization API for Java and ImageJ. BMC Bioinformatics, 2010. 11: p. 274.

16. Schneider, C.A., W.S. Rasband, and K.W. Eliceiri, NIH Image to ImageJ: 25 years of image analysis. Nat Methods, 2012. 9(7): p. 671-5.

17. Bosma, M., et al., Re-evaluating lipotoxic triggers in skeletal muscle: relating intramyocellular lipid metabolism to insulin sensitivity. Prog Lipid Res, 2012. 51(1): p. 36-49.

18. Prats, C., et al., An optimized histochemical method to assess skeletal muscle glycogen and lipid stores reveals two metabolically distinct populations of type I muscle fibers. PLoS One, 2013. 8(10): p. e77774.

19. Gocze, P.M. and D.A. Freeman, Factors underlying the variability of lipid droplet fluorescence in MA10 Leydig tumor cells. Cytometry, 1994. 17(2): p. 151-8.

20. Daemen, S., et al., Microscopy tools for the investigation of intracellular lipid storage and dynamics. Mol Metab, 2016. 5(3): p. 153-63.

21. Schermelleh, L., R. Heintzmann, and H. Leonhardt, A guide to super-resolution fluorescence microscopy. J Cell Biol, 2010. 190(2): p. 165-75. 


\section{CHAPTER 4}

\section{Decoration of intramyocellular lipid droplets with PLIN5 modulates fasting-induced insulin resistance and lipotoxicity in humans}

Anne Gemmink ${ }^{*}$, Madeleen Bosma*, Helma J. Kuijpers, Joris Hoeks, Gert Schaart, Marc A.M.J. van Zandvoort, Patrick Schrauwen, Matthijs K.C. Hesselink

Published in Diabetologia 2016; 59:1040-1048

*These authors contributed equally to this work 


\section{ABSTRACT}

Aims/hypothesis: In contrast to insulin resistant subjects, insulin sensitive athletes possess high intramyocellular lipid content (IMCL), good mitochondrial function and high Perilipin 5 (PLIN5) content, suggesting a role for PLIN5 in benign IMCL storage. We hypothesized a role for PLIN5 in modulating fasting-mediated insulin resistance.

Methods: Twelve males were fasted for 60 hours before and after which muscle biopsies were taken and stained for LDs, PLIN5 and laminin. Confocal microscopy images and were analyzed for LD size, number, PLIN5 association and subcellular distribution.

Results: Fasting elevated IMCL content 2.8 fold and reduced insulin sensitivity (a drop of 55\%). Individuals with the most prominent increase in IMCL, dropped least in insulin sensitivity $(r=0.657$; $p=0.028)$ and mitochondrial function $(r=0.896 ; p=0.006)$. While upon fasting, PLIN5 gene expression or protein content in muscle homogenates was unaffected, microscopy analyses revealed that the fraction of PLIN5 associated with LDs (PLIN5+) increased significantly (+26\%) upon fasting, suggesting PLIN5 redistribution. The significant increase in LD number (+23\%) and size (+23\%) upon fasting was entirely accounted for by PLIN5+ LDs, not by LDs devoid of PLIN5. Also the association between IMCL storage capacity and insulin resistance and mitochondrial dysfunction was only apparent for PLIN5+ LDs.

Conclusion/interpretation: Fasting results in subcellular redistribution of PLIN5, and promotes the capacity to store excess fat in larger and more numerous PLIN5 decorated LDs. This associates with blunting of fasting-induced insulin resistance and mitochondrial dysfunction, suggesting a role for PLIN5 in the modulation of fasting-mediated lipotoxicity. 


\section{INTRODUCTION}

Excess lipid can be stored ectopically as triacylglycerol in lipid droplets (LDs) in e.g. skeletal muscle. This is often associated with compromised myocellular insulin sensitivity [1-3]. Rodent studies revealed that a high fat diet increases intramyocellular lipid (IMCL) content and promotes insulin resistance [4]. Acute elevations of lipid availability in humans profoundly augments IMCL storage and insulin resistance [5]. How excess fat storage in muscle affects insulin sensitivity, however, is not yet clear.

Excess fat in muscle is stored in LDs containing predominantly neutral lipids like triacylglycerol and cholesteryl esters. LD size [6], subcellular distribution [7-9], composition [10] and interaction of LDs with other cellular organelles like mitochondria [11] have all been postulated to contribute to the observed association between $\mathrm{IMCL}$ storage and insulin resistance. Paradoxically, however, stimulating muscle fat storage by overexpressing diacylglycerol-o-acyltransferase-1 (DGAT1) profoundly augments IMCL storage [12], without compromising insulin sensitivity [13, 14]. Moreover, endurance trained athletes have high levels of IMCL whilst being very insulin sensitive [15] and are less sensitive to acute lipid-induced insulin resistance upon lipid infusion [5]. In search for a unifying factor in conditions of maintained insulin sensitivity despite the presence of high levels of IMCL, we noticed that in all conditions when insulin sensitivity was maintained even though IMCL was high or elevated, gene expression or protein content of PLIN5 was increased $[13,16,17]$.

PLIN5 is a member of the perilipin LD coat proteins. PLIN5 is predominantly expressed in metabolically active tissues [18-20] where it appears to regulate oxidative LD lipolysis [21] and hence affects LD size. To examine the effect of PLIN5 on lipid-induced insulin resistance we overexpressed PLIN5 in rat skeletal muscle, and observed a profound increase in intramyocellular LD content while insulin stimulated glucose uptake was not impaired [22]. In line with this observation, it has recently been shown that whole body deletion of PLIN5 in mice, reduced insulin mediated glucose uptake [23]. Of the other perilipin family members present in muscle, PLIN2 has also been reported to promote retaining lipids in the LD and to prevent HFD induced reductions in insulin sensitivity [24] whereas PLIN3 has recently been associated with fat oxidation in cultured muscle cells [25]. As of yet, PLIN5 however is the only member of the perilipin family that has been directly linked to LD turnover and fat oxidation in multiple models of insulin resistance [26]. Thus, we focused on PLIN5 and hypothesized that elevation of PLIN5 contributes to maintenance of insulin sensitivity under conditions of abundance of lipids. Under pathophysiological conditions like type 2 diabetes, insulin resistance of adipose tissue results in disinhibition of adipose tissue lipolysis by insulin. Thus, the pathological state of type 2 diabetes creates a situation in which glucose, insulin and plasma non- 
esterified fatty acids are elevated. Although the putative effects of insulin on lipolysis of myocellular LDs has not been studied in detail, it has been reported that insulin decreases gene expression of the major triglyceride lipase, ATGL [27]. Moreover, transcriptional activation and phosphorylation of members of the perilipin family is reportedly affected by elevated insulin and glucose levels [28]. We hence studied the putative role of PLIN5 in the physiological regulation of insulin sensitivity, in a model in which lipid overload was physiologically induced and in which blunted insulin sensitivity could not be attributed to secondary effects of long term pathophysiological consequences of insulin resistance, like hyperglycemia or hyperinsulinemia. Therefore, we employed our studies in a model of prolonged fasting (60 hours) in humans, which we previously published [29]. In this study, prolonged fasting resulted in a profound drop in insulin sensitivity and increased IMCL content, while insulin and glucose levels were in the low end of the physiological range. Interestingly, rodent data indicate that - at least in cardiac muscle - a prolonged fast was paralleled by increased myocardial fat content and induction of PLIN5 [19]. Therefore, we hypothesized a role for PLIN5 in modulating fasting-induced insulin resistance.

As we previously observed that genetically promoting PLIN5 resulted in increased LD size [22], we combined studies in whole muscle homogenates with detailed quantitative confocal fluorescence microscopy based morphometric permitting analysis of individual LDs under fed- and fasted conditions.

\section{METHODS}

\section{Ethics statement}

The study protocol was approved by the institutional Medical Ethical Committee. All subjects $(n=11)$ gave written informed consent prior to participating in the study. This study has been registered in the 'Nederlands Trial Register' (www.trialregister.nl) with registration number NTR 2042. The plasma data presented in this paper originate from previously published work [29].

\section{Subjects and study protocol}

A detailed protocol of the present study has previously been published elsewhere [29]. In brief, twelve young, normoglycemic lean males were studied in the fasted (60 hours fast) or fed state, in a randomized cross-over design with a 2-week washout period in between. None of the subjects was 
engaged in sports $>2$ hours per week. To ensure compliance to the intervention, the entire study was performed in a respiration chamber. In the fasted state only calorie-free drinks were consumed. In the fed state subjects were fed in energy balance (with 50-35-15 En\% consumed as carbohydrates, fat and protein, respectively).

Post-intervention (60 hours fast or fed), a hyperinsulinemic-euglycemic clamp was performed (40 $\mathrm{mU} \mathrm{m} \mathrm{min}^{-1}$ insulin combined with infusion of $\left[6,6-{ }^{2} \mathrm{H}_{2}\right]$ glucose to measure rates of glucose disposal (Rd). Prior to the clamp, a biopsy was taken from the $\mathrm{m}$. vastus lateralis. One portion was processed for mitochondrial capacity assays (high-resolution respirometry to assess ADP-driven state 3 respiration in permeabilized muscle fibers using octanoyl-CoA, glutamate and succinate as substrates). Maximal uncoupled respiration was measured upon FCCP titration. Oxygen flux was normalized to mitochondrial content based upon mtDNA copy number) [29]. Another portion of the sample was frozen directly in melting isopentane, and stored at $-80^{\circ} \mathrm{C}$ for qRT-PCR analysis, Western blotting and quantitative (immuno)fluorescence microscopy. Muscle insulin sensitivity was expressed as insulin sensitivity index ( $\mathrm{S}_{\mathrm{i}}$-index) and was calculated as (Rd insulin stimulated-Rd basal)/(plasma insulin*plasma glucose). Confocal immunofluorescence is based upon 9 subjects. Characteristics of all subjects are presented in Table 1.

Table 1. Subject characteristics $(n=12)$

\begin{tabular}{lc}
\hline Parameter & Mean \pm SEM \\
\hline Age (years) & $23.6 \pm 1.0$ \\
Body weight $(\mathrm{kg})$ & $78.5 \pm 2.5$ \\
Fat-free mass $(\mathrm{kg})$ & $65.9 \pm 1.8$ \\
Height $(\mathrm{m})$ & $1.86 \pm 0.02$ \\
$\mathrm{BMI}\left(\mathrm{kg} / \mathrm{m}^{2}\right)$ & $22.6 \pm 0.5$ \\
Maximal aerobic capacity $\left(\mathrm{ml} \mathrm{O} / \mathrm{kg}_{\mathrm{FFM}} / \mathrm{min}\right)$ & $57.5 \pm 1.5$ \\
\hline
\end{tabular}

\section{Quantitative Real-Time PCR}

RNA was isolated from approximately $30 \mathrm{mg}$ muscle tissue essentially according to Chomczynski et al. [30]. Quantitative real-time RT-PCR was performed as described previously [31, 32]. Primers and probes are presented in Supplementary Table 1. Gene expression data of PLIN5 was normalized over RPLPO. 


\section{Western Blots}

Western blots were performed using antibodies against PLIN5 (Progen GP31; Progen Biotechnik, Heidelberg, Germany) and SR-actin (A-2172; Sigma, St. Louis, USA). Infra-red tagged secondary antibodies (IRDye; LI-COR, Lincoln, NE) were used to visualize and quantify the relevant protein bands (Odyssey Infrared Imaging system, LI-COR Biosciences, Westburg, Leusden, The Netherlands).

\section{Histochemical analysis}

Fresh cryosections $(7 \mu \mathrm{m})$ of samples from the fed and fasted state were cut and thaw-mounted on a single glass slide to minimize variety in staining intensity. Initial quantification of IMCL was performed by oil-red-O staining [33]. To allow valid LD morphometry, sections displaying freezing damage were discarded. As a resultant, full morphometric analyses was performed on $3595 \pm 522$ and $4766 \pm 489$ LDs per subject $(n=9)$ in the fed and fasted state, respectively. For quantification of PLIN5, sections were fixed in $3.7 \%$ formaldehyde in PBS for 30 minutes, washed for 5 minutes with PBS, blocked for 45 minutes with blocking buffer $(150 \mathrm{mmol} / \mathrm{L} \mathrm{NaCl}, 20 \mathrm{mmol} / \mathrm{L}$ Tris $\mathrm{pH} 6.8$ and 2\% BSA) and permeabilized with 0.25\% Triton-X 100 (648466, Merck, Darmstadt, Germany). Subsequently, sections were washed with PBS for 5 minutes followed by a 1-hour incubation with primary antibodies against Laminin (L9393, Sigma, St. Louis, USA) and PLIN5 (GP31; Progen Biotechnik, Heidelberg, Germany) in blocking buffer at room temperature. Lipid droplets were visualized using Bodipy ${ }^{\circledR}$ 493/503 (Molecular Probes, Leiden, The Netherlands). Sections were incubated for 1.5 hour along with the appropriate secondary antibodies conjugated with Alexa Fluor 405 or Alexa Fluor 647 (Invitrogen, Groningen, The Netherlands) at $37^{\circ} \mathrm{C}$ and mounted with Mowiol (5886, Merck, Darmstadt, Germany).

\section{Confocal image acquisition and analysis}

Multiple sliced Z-stacks were acquired on a Leica TCS SPE confocal microscope using a 63x 1.3 N.A. oil immersion objective with a 1.1 optical zoom at 2048 by 2048 pixels resulting in a pixel size of 77 by $77 \mathrm{~nm}$. Laminin-Alexa Fluor 405, Bodipy ${ }^{\circledR} 493 / 503$ and PLIN5-Alexa Fluor 647 were imaged using the $405 \mathrm{~nm}, 488 \mathrm{~nm}$ and $635 \mathrm{~nm}$ laser lines, respectively. Emission was detected between 415-460 $\mathrm{nm}, 500-560 \mathrm{~nm}$ and 650-750 nm for respectively Laminin-Alexa Fluor 405, Bodipy ${ }^{\circledR}$ 493/503 and PLIN5-Alexa Fluor 647. To permit valid quantification of the fluorescence signal from PLIN5-Alexa Fluor 647, pixel saturation was prevented. Z-stacks were acquired for deconvolution purposes 
(Huygens Essential software, Scientific Volume Imaging B.V., Hilversum, The Netherlands) and analyzed using ImageJ [34]. Intensity of PLIN5 staining was analyzed on the non-deconvoluted images. LDs coated with PLIN5 (co-localization with PLIN5) and devoid of PLIN5 were quantified using a custom written routine in Matlab R2012a (The Mathworks, Inc., Natick, Massachusetts, USA).

\section{Statistical analyses}

Results are presented as mean \pm SEM. Statistical analyses were performed using SPSS version 21.0 (SPSS, Chicago, IL, USA). Statistical comparisons between conditions were performed using paired Ttests. Pearson's correlation coefficients were used to describe the linear association between variables. $p<0.05$ was considered statistically significant.

\section{RESULTS}

\section{Plasma parameters}

We previously published a significant increase in circulatory free fatty acids levels ( $\sim$-fold) and a significant drop in plasma glucose values, insulin levels and insulin sensitivity (glucose infusion rate and $\mathrm{S}_{\mathrm{i}}$-index) upon fasting [29]. These data indicate that fasting created the warranted condition of profound insulin resistance and increased circulatory free fatty acids in the absence of hyperglycemia or hyperinsulinemia for the present study.

\section{Intramyocellular lipid content and insulin resistance}

Fasting significantly augmented IMCL content $(1.67 \pm 0.32$ in the fed state to $4.60 \pm 0.72 \mathrm{AU}$ after fasting) [29]. Interestingly, the increase in IMCL (IMCL in the fasted state minus IMCL in the fed state) upon fasting correlated positively $(r=0.657 ; p=0.028)$ with the drop $\left(S_{i}\right.$-index in the fasted state minus the $S_{i}$-index in the fed state) in the $S_{i}$-index (Figure 1), suggesting that promoting IMCL storage capacity ameliorates fasting-induced insulin resistance. 


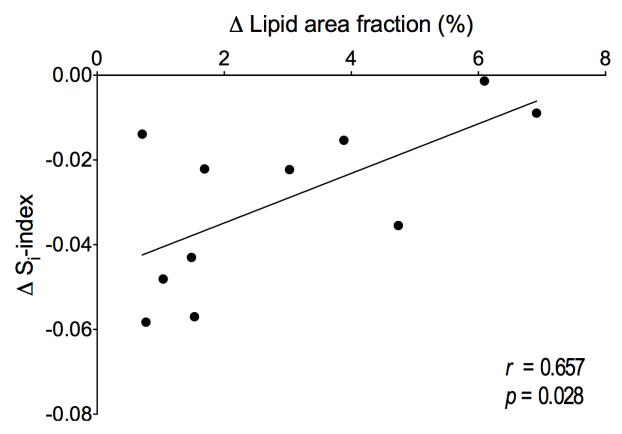

Figure 1. Correlation of the change in $\mathrm{IMCL}$ content and $\mathrm{S}_{\mathrm{i}}$-index

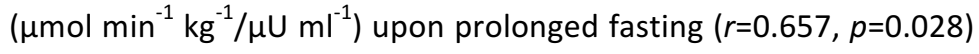
$(n=11)$, suggesting that promoting the capacity to store lipids as $\mathrm{IMCL}$ ameliorates fasting-induced insulin resistance. This correlation appears to be driven by PLIN5 coated lipid droplets (Figure 6A and 6B).

\section{Morphometric analysis}

As changes in LD size [6] may contribute to the development of insulin resistance we employed a microscopical approach to examine LD size and number. Fasting promoted LD size $\left(0.26 \pm 0.01 \mu \mathrm{m}^{2}\right.$ vs. $\left.0.32 \pm 0.01 \mu^{2} ; p<0.01\right)$ and number $\left(0.039 \pm 0.004 \mu \mathrm{m}^{-2}\right.$ vs. $\left.0.048 \pm 0.003 \mu \mathrm{m}^{-2} ; p<0.05\right)$ (Figure $2 \mathrm{~A}$ and B). LD size distribution analyses revealed a drop in the percentage of small $\left(\leq 0.20 \mu \mathrm{m}^{2}\right)$ LDs upon fasting, whereas the percentage of large $\left(\geq 0.35 \mu \mathrm{m}^{2}\right)$ LDs significantly increased (Figure $2 \mathrm{C}$ ).

$\mathbf{A}$

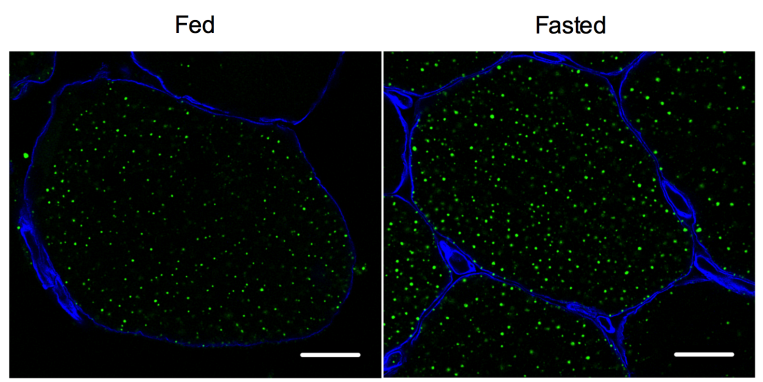

B
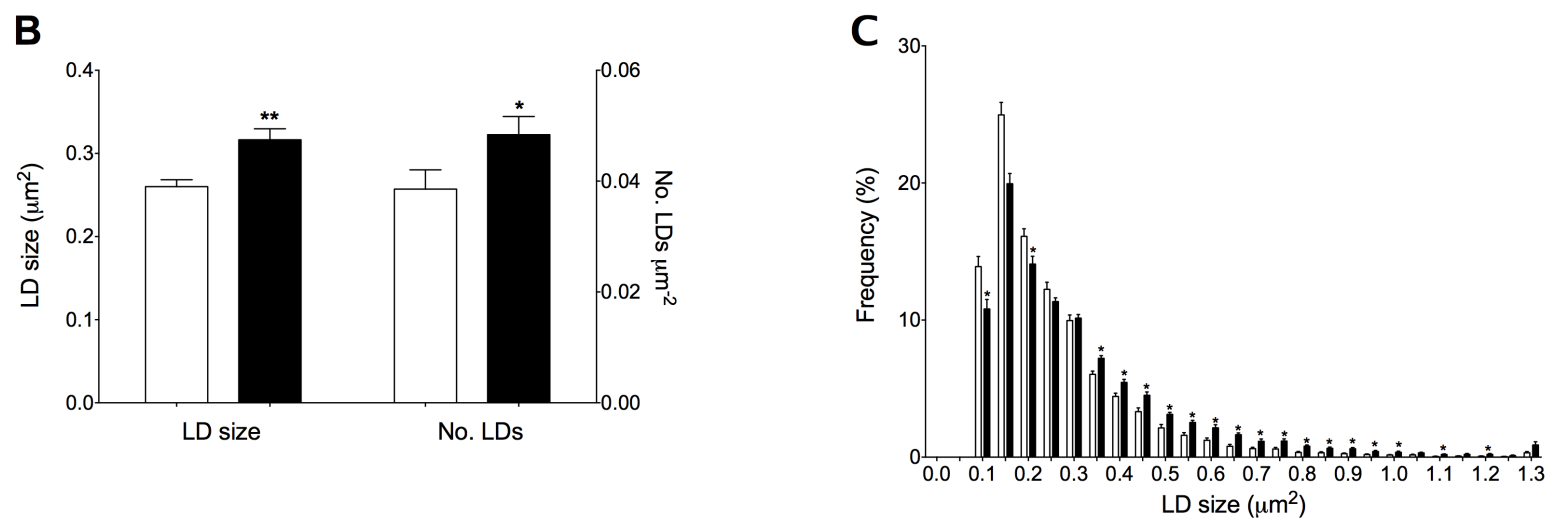

Figure 2. Lipid droplet size and number increased upon prolonged fasting. (A) Representative images at 70 times magnification of muscle fibers from the fed and fasted state. LDs are stained in green and cell membranes in blue. (B) Quantification of LD size (left) and number (right) relative to cell area. (C) Frequency distribution of LD size. Data are based upon examination of $3595 \pm 522$ and $4766 \pm 489$ LDs per subject ( $n=9)$ in the fed and fasted state and presented as mean \pm SEM, ${ }^{*} p<0.05$ or ${ }^{* *} p<0.01$ vs. fed state. Open bars refer to the fed state, black bars to the fasted state. Scale bare equals $15 \mu \mathrm{m}$. 


\section{PLIN5 content on individual LDs}

Conventional analysis of gene expression and protein content of PLIN5 in whole muscle lysates revealed no effect of fasting on PLIN5 mRNA (1.04 \pm 0.13 vs. $1.11 \pm 0.11 ; p=0.72)$ or protein content (10.4 \pm 3.1 A.U. vs. 10.3 \pm 2.8 A.U.; $p=0.99$ ) (Figure $3 \mathrm{~A}$ and $3 B$ respectively). Muscle lysates were made from sections with equal muscle fiber type distribution between type I and type II muscle fibers in the fed and fasted state $(50 \pm 2 \%$ and $52 \pm 4 \%$ type I fibers in the fed and fasted state, respectively and $50 \pm 2 \%$ and $48 \pm 4 \%$ type II fibers in the fed and fasted state, respectively). PLIN5 is known to be most abundantly expressed in type I fibers. Hence we selected predominantly type I fibers for quantitative high-resolution confocal immunofluorescence microscopy. We simultaneously quantified individual LD size and PLIN5 protein content (Figure 4A) by microscopy. In line with the observation in whole muscle lysates, we observed that total PLIN5 protein content was unaffected by fasting $(2181 \pm 202$ A.U. vs. $2443 \pm 312$ A.U.; $p=0.276$ in the fed and fasted state, respectively, Figure 4 B). To avoid bias introduced by a potential fasting induced fiber type shift, a similar percentage of type I muscle fibers in the fed $(82 \pm 6 \%)$ and in the fasted state $(87 \pm 6 \%)$ was examined.

A

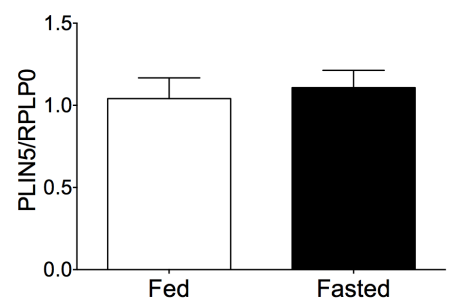

B

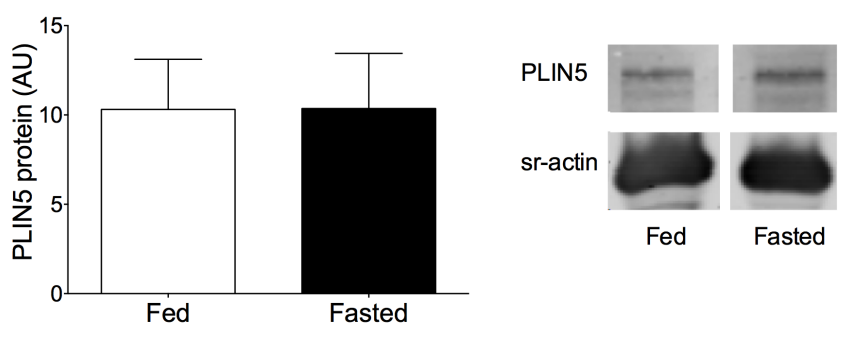

Figure 3. (A) PLIN5 gene expression ( $n=12)$ and (B) protein content $(n=11)$ measured in whole muscle lysates in the fed and the fasted state. Data are presented as mean \pm SEM.

Given the putative role of PLIN5 in controlling lipolysis and sequestering fatty acids in LDs by covering the LD surface [35], we examined the fraction of PLIN5 protein associated with LDs. Although total PLIN5 content was not affected by fasting, the fraction of PLIN5 protein directly associated with LDs significantly increased upon fasting from $14.9 \pm 2.2 \%$ to $18.7 \pm 1.3 \%$ (figure $4 \mathrm{C}$ ), suggesting a fasting-induced redistribution of PLIN5 from cytosolic sites to the LD surface. Using this novel microscopical approach we could also delineate LDs with high levels of PLIN5 protein (PLIN5+) from LDs devoid of PLIN5 (PLIN5-). 
A

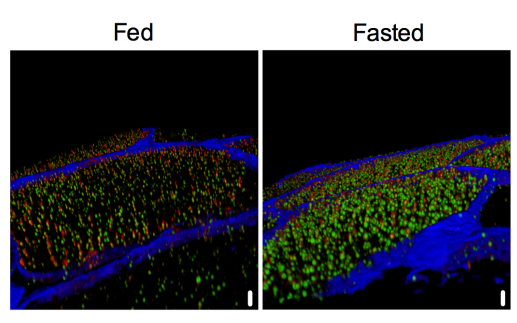

B

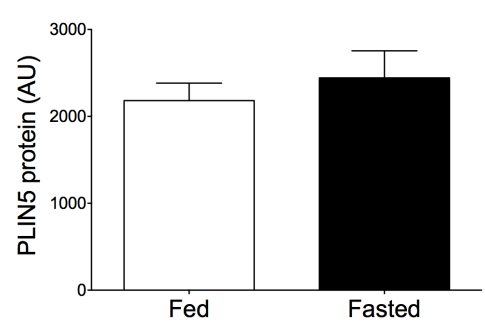

C

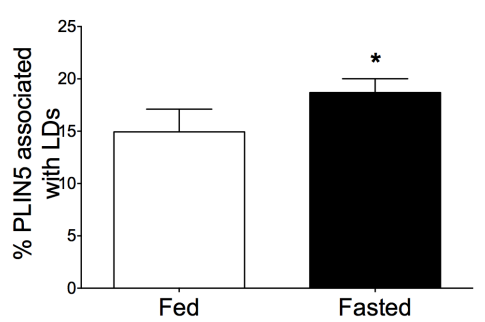

Figure 4. Representative 3D images of LDs (green) and PLIN5 protein localization (red) in the fed-and fasted state. (B) Average PLIN5 protein content in muscle fibers measured as intensity of PLIN5 staining. (C) Fraction of PLIN5 protein content associated with LDs. Data are based upon examination of $3595 \pm 522$ and $4766 \pm 489$ LDs per subject $(n=9)$ in the fed and fasted state and presented as mean $\pm S E M, * p<0.05$ vs. fed state. Scale bare equals $7 \mu \mathrm{m}$.

Upon making the distinction between PLIN5+ and PLIN5- LDs, we observed that the fasting-mediated increase in LD size as well as number was entirely accounted for by PLIN5+ LDs (Figure 5A and B), whereas PLIN5- LDs did not change in size nor number (Figure 5A and B). Although these data do not permit statements on causality, they indicate involvement of PLIN5 in the fasting-mediated increase in LD size and number.

\section{A}

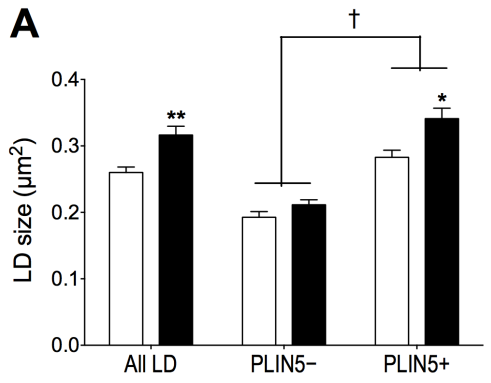

B

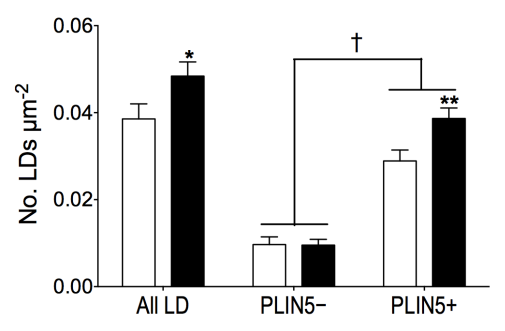

Figure 5. Changes in (A) LD size and (B) number are accounted for by PLIN5+ LDs. Data are based upon examination of $3595 \pm 522$ and $4766 \pm 489$ LDs per subject $(n=9)$ in the fed and fasted state and are presented as mean \pm SEM, ${ }^{*} p<0.05$ or ${ }^{* *} p<0.01$ vs. fed state and t $p<0.001$ PLIN5+ vs. PLIN5- LDs. Open bars refer to the fed state, black bars to the fasted state. 
Upon making the distinction between PLIN5+ and PLIN5- LDs, we also observed that the correlation between the change in myocellular fat deposition (change in IMCL) and insulin sensitivity which was observed upon inclusion of all LDs $(r=0.657, p=0.028$, Figure 1$)$. If only PLIN5+ LDs were taken into account, this correlation was less strong $(r=0.587)$ than upon inclusion of all $L D s$, but still approached the level of significance ( $p=0.096$, Figure $6 \mathrm{~A})$. This in contrast to LDs devoid of PLIN5 for which this correlation had completely vanished ( $r=-0.381, p=0.311$, Figure 6B).

A

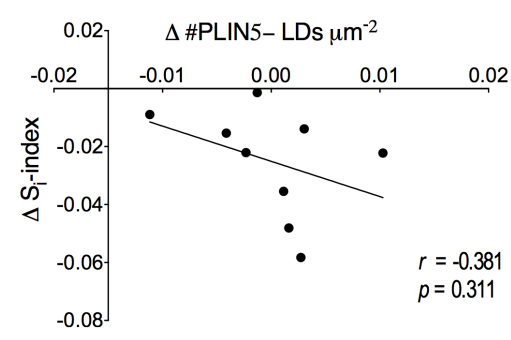

B

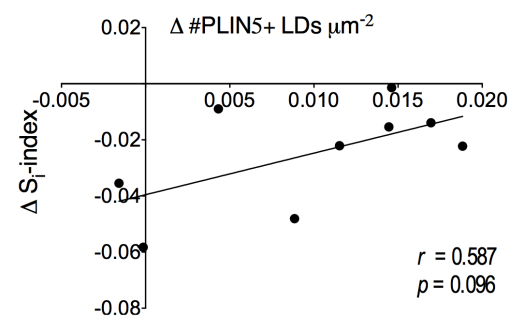

Figure 6: Correlations of the fasting-induced changes (fasted-fed) in (A) the number of PLIN5+ LDs $\left(\mu m^{-2}\right)(r=0.578, p=0.096)$ and (B) the number of PLIN5- LDs $\left(\mu \mathrm{m}^{-2}\right)(r=-0.381, p=0.311)$ with the reduction in

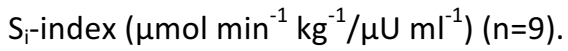

Myocellular fat accumulation is not only associated with compromised insulin sensitivity, but also with the development of mitochondrial dysfunction [29], a process referred to as mitochondrial lipotoxicity [36]. Using a variety of substrates, we observed that the drop in mitochondrial function (ADP-driven state 3 respiration, as well as FCCP-mediated maximal uncoupled respiration) upon fasting correlated with the increase in LD size upon fasting $(r=0.896 ; p=0.006$ for octanoyl-CoA; $r=0.795 ; p=0.033$ for octanoyl-CoA with glutamate; $r=0.873 ; p=0.010$ for octanoyl-CoA with glutamate and succinate and $r=0.853 ; p=0.015$ for maximal uncoupled respiration, Figure 7A, 7D, 7G and $7 J$ respectively). These correlations were maintained if only PLIN5+ LDs were taken into account (Figure 7B, 7E, 7H and 7K), but strikingly were absent for PLIN5- LDs (Figure 7C,7F, 7I, 7L). 
A

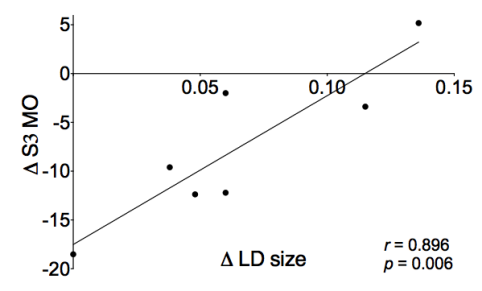

D

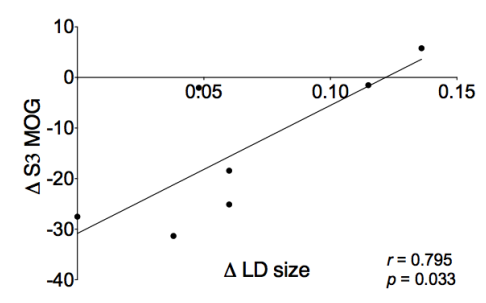

G

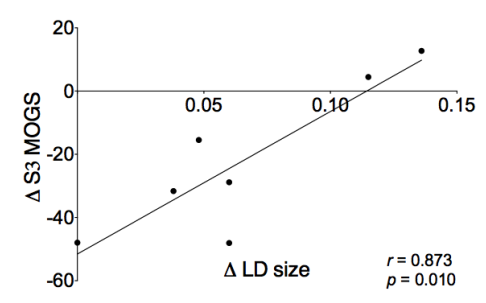

J

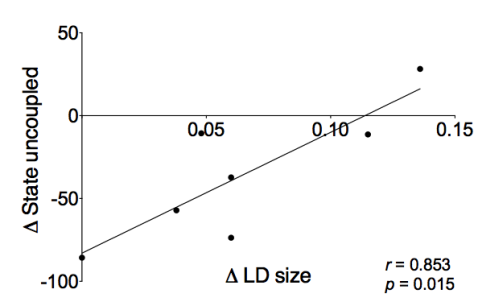

B

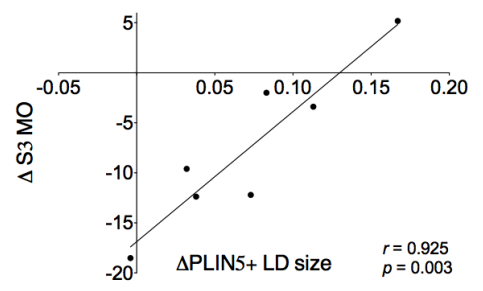

E

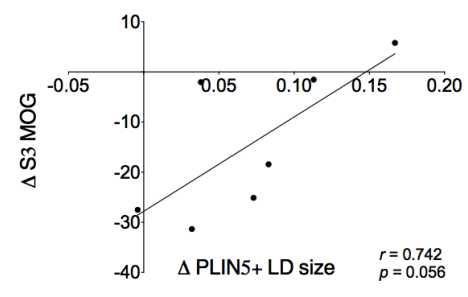

H

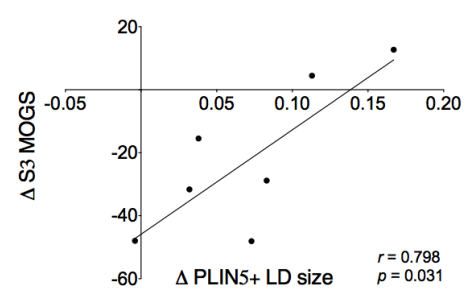

K

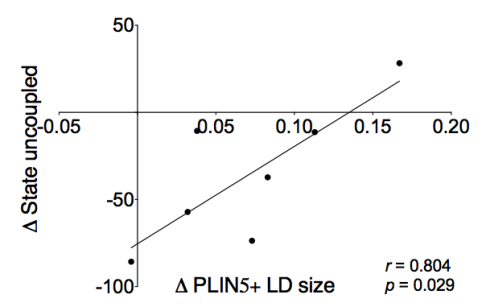

C

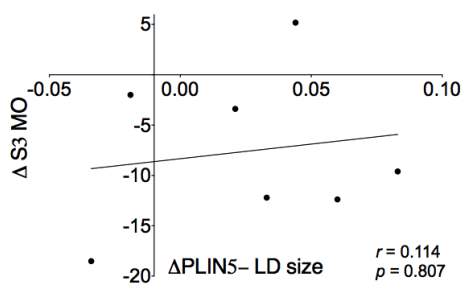

F

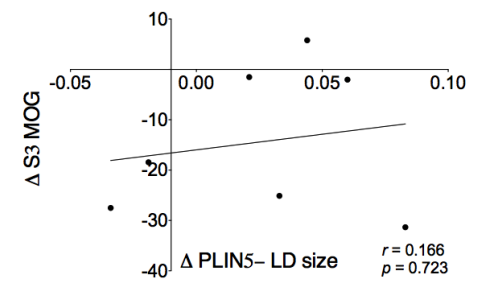

I

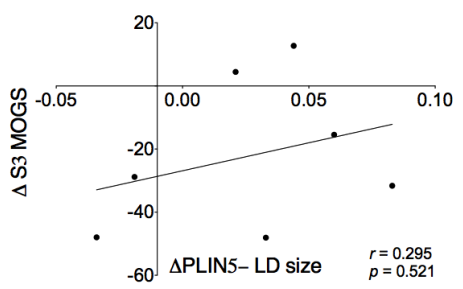

L

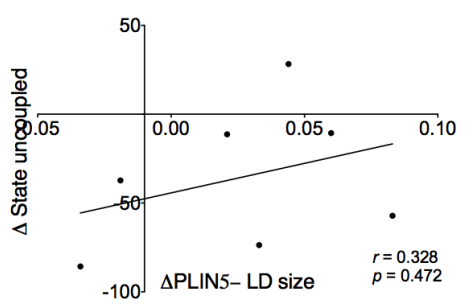

Figure 7: Correlations of the fasting-induced changes in LD size $\left(\mu \mathrm{m}^{2}\right)$ of $(A)$ all LDs $(r=0.887, p=0.008),(B)$ PLIN5+ LDs $(r=0.940, p=0.002)$ and (C) PLIN5- LDs $(r=0.151, p=0.746)$ with changes in mitochondrial ADP-driven

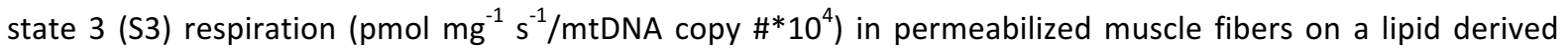
substrate octanoyl-CoA (MO), (D-F) octanoyl-CoA+glutamate (MOG), (G-I) octanoyl-CoA+glutamate+succinate (MOGS) and $(\mathrm{J}-\mathrm{L})$ during maximal uncoupling $(\mathrm{n}=7)$. 


\section{DISCUSSION}

Here we studied the hypothesis that the lipid droplet (LD) coat protein PLIN5 increases upon fasting and that PLIN5 is involved in the maintenance of insulin sensitivity under conditions of lipid overload. To this end, we took advantage of a physiological model of insulin resistance in humans, being prolonged (60 hours) fasting. We showed that the previously reported increase in IMCL upon fasting [29] originates from an increase in both number and size of LDs. Although total PLIN5 content was not affected by fasting, a subcellular redistribution of PLIN5 was observed after fasting, resulting in increased coverage of the LDs with PLIN5. Interestingly, the increase in LD size and number was entirely explained by increases in LD size and number of LDs coated with PLIN5. No such change was observed in droplets devoid of PLIN5. Moreover, we showed that subjects capable of increasing muscle fat content most upon fasting, dropped least in insulin sensitivity and mitochondrial function. Also, this observation was entirely accounted for by LDs coated with PLIN5. Jointly, these data indicate that the fasting-mediated increase of PLIN5 on the LD surface is part of the adaptive response to fasting to alleviate lipid-induced insulin resistance and to maintain mitochondrial function.

Most of our knowledge on the response of PLIN5 to fasting originates from cardiac muscle of rodent studies [18-20]. These papers indicate that fasting promotes PLIN5 gene expression and protein content in cardiac muscle, whereas in mouse skeletal muscle ( $\mathrm{m}$. gastrocnemius and $\mathrm{m}$. soleus) PLIN5 protein content appeared to be unaffected upon a 24 hour fast [18]. The effect of fasting on PLIN5 content in human skeletal muscle has not yet been examined. In line with animal data in skeletal muscle we observed that total PLIN5 content in muscle was unaffected upon fasting. Rather, fasting resulted in redistribution of PLIN5 from cytosolic pools to the LD surface. The fraction of the LDs coated with PLIN5 increased significantly upon fasting, as well as LD size and the fraction of PLIN5 protein content associated with the LD.

Although PLIN5 is best known as a LD coat protein, subcellular fractionation studies revealed the presence of PLIN5 in cytosolic fractions [19]. At present, it is not known if the cytosolic PLIN5 pool represents unbound PLIN5 or PLIN5 which is somehow located to -yet to be identified- subcellular structures. Hence, it is not known if the observed redistribution of PLIN5 in fact is a targeted translocation from a defined locus to the LD, or if it represents expansion of nano-scaled LDs coated with PLIN5, that did not exceed the lower detection limit of the microscope in the fed state (and hence were considered cytosolic) but became detectable upon fasting. It should be noted, however, that fasting resulted in a pool of largely sized LDs decorated with PLIN5 (PLIN5+ LDs) whereas the small sized LDs were devoid of PLIN5. This reduces the likelihood that the pool of cytosolic PLIN5 in 
fact represents a pool bound to nano-scaled LDs and favors the concept of fasting-mediated redistribution of PLIN5.

In the present model of physiological insulin resistance, PLIN5+ LDs were more numerous and larger than PLIN5- LDs. Hence, LD expansion may originate from coating of the LD with PLIN5 and its inhibitory effect on myocellular LD lipolysis, either by binding to the major triglyceride lipase ATGL [37] or by binding to the co-activator of ATGL, being CGI-58 [38]. In trained athletes, high levels of IMCL and high insulin sensitivity go along with high levels of PLIN5 $[16,17]$, indicating that PLIN5 may modulate insulin sensitivity under conditions of high lipid load. Importantly, delineation of PLIN5+ and PLIN5- LDs for the first time provides experimental support to the notion that increased myocellular fat content does not necessarily impede insulin sensitivity, provided that the excess fat is stored in PLIN5+ LDs. Thus, expanding the pool of LDs decorated with PLIN5 could be considered an adaptive response to fasting to maintain LD dynamics and prevent insulin resistance. Interestingly, we recently showed that individual LDs coated with PLIN5 sequester more bioactive insulin desensitizing lipids than LDs devoid of PLIN5 [39], providing a possible explanation as to why storage of excess fat in PLIN5+ LDs ameliorates lipid-induced insulin resistance.

PLIN5 has also been hypothesized to modulate lipotoxicity by promoting interaction and/or intimate physical association of LDs with mitochondria $[21,40]$ and to promote oxidative gene expression [40] to facilitate mitochondrial degradation of the fatty acids released from LD lipolysis. Accordingly, PLIN5 may be involved in the prevention against mitochondrial lipotoxicity. In that respect it is of interest to note that the fasting-mediated drop in mitochondrial function (measured as state 3 ADP driven mitochondrial oxygen uptake and FFCP mediated maximal uncoupled respiration on a variety of substrates) correlated positively with the fasting-mediated increase in LD size. Upon making the distinction between PLIN5+ and PLIN5- LDs also this correlation appeared to originate from PLIN5+ LDs and was completely absent for PLIN5- LDs, for state 3 respiration as well as for maximally uncoupled respiration, irrespective of the substrate used. This again supports the notion that decoration of LDs with PLIN5 may prevent mitochondrial lipotoxicity upon fasting, possibly by expanding the capacity for inert lipid storage. This notion substantiates our previously reported observation that in muscle of Zucker diabetic fatty rats PLIN5 protein content correlates positively with mitochondrial function [40]. Jointly, our observation that expansion of PLIN5+ LDs ameliorates mitochondrial lipotoxicity and lipid-induced insulin resistance are in line with rodent data indicating a protective role of PLIN5 in lipid-induced insulin resistance $[22,23]$ and hepatic lipotoxicity [41].

Next to the methodological advance permitting analysis at the level of individual LDs, a major strength of the present study is that, in contrast to most other models of insulin resistance or type 2 
diabetes, its design permits conclusions on the role of PLIN5 at the very early stages of insulin resistance development. We studied drug naïve, healthy, lean and young males in a situation when glucose and insulin are well within the normal physiological range. Thus, our observations cannot be attributed to consequences secondary to the pathological state that parallels most models of insulin resistance and hence likely reflect physiological adaptive responses to the very early insulin resistant state. Maintenance of these adaptive responses may blunt the pathogenesis of insulin resistance.

Myocellular lipid handling is affected by muscle fiber type distribution as well as gender [42]. In the present study, we carefully considered muscle fiber type distribution as a potential confounder. For data on whole muscle cell lysates (PLIN5 protein content and gene expression) we ensured equal distribution of type I and type II muscle fibers in the fed and the fasted state. Under untreated and physiological conditions, type I fibers store most lipid and express PLIN5 most abundantly. Hence, we purposely focused our microscopy on type I muscle fibers, with a similar fraction type I fibers being examined in the fed and fasted state. With respect to gender, it is important to note that in the present study we only examined males. Therefore, extrapolation of the present findings to females must be done with care, especially given the gender differences previously reported for myocellular lipid handling [42], lipid-induced insulin resistance [43], response to fasting [44] and PLIN5 content [45].

In conclusion, the present study shows that PLIN5 protein content did not increase in human skeletal muscle upon fasting, however, fasting resulted in a redistribution of PLIN5 from a non-LD-associated cytosolic pool towards the LD. The ability to store excess fat in skeletal muscle in PLIN5+ LDs upon a prolonged fast associates with blunting of fasting-induced insulin resistance and mitochondrial dysfunction. These data are in support of a role of PLIN5 in mitigation of fasting-mediated lipotoxicity.

\section{REFERENCES}

1. Coen, P.M. and B.H. Goodpaster, Role of intramyocelluar lipids in human health. Trends Endocrinol Metab, 2012. 23(8): p. 391-8.

2. Pan, D.A., et al., Skeletal muscle triglyceride levels are inversely related to insulin action. Diabetes, 1997. 46(6): p. 983-8.

3. Goodpaster, B.H., et al., Intramuscular lipid content is increased in obesity and decreased by weight loss. Metabolism, 2000. 49(4): p. 467-72.

4. Storlien, L.H., et al., Influence of dietary fat composition on development of insulin resistance in rats. Relationship to muscle triglyceride and omega-3 fatty acids in muscle phospholipid. Diabetes, 1991. 40(2): p. 280-9.

5. Phielix, E., et al., High oxidative capacity due to chronic exercise training attenuates lipid-induced insulin resistance. Diabetes, 2012. 61(10): p. 2472-8. 
6. He, J., B.H. Goodpaster, and D.E. Kelley, Effects of weight loss and physical activity on muscle lipid content and droplet size. Obes Res, 2004. 12(5): p. 761-9.

7. Lally, J.S., et al., Subcellular lipid droplet distribution in red and white muscles in the obese Zucker rat. Diabetologia, 2012. 55(2): p. 479-88.

8. Nielsen, J., et al., Increased subsarcolemmal lipids in type 2 diabetes: effect of training on localization of lipids, mitochondria, and glycogen in sedentary human skeletal muscle. Am J Physiol Endocrinol Metab, 2010. 298(3): p. E706-13.

9. Samjoo, I.A., et al., Markers of skeletal muscle mitochondrial function and lipid accumulation are moderately associated with the homeostasis model assessment index of insulin resistance in obese men. PLoS One, 2013. 8(6): p. e66322.

10. Greenberg, A.S., et al., The role of lipid droplets in metabolic disease in rodents and humans. J Clin Invest, 2011. 121(6): p. 2102-10.

11. Walther, T.C. and R.V. Farese, Jr., Lipid droplets and cellular lipid metabolism. Annu Rev Biochem, 2012. 81: p. 687-714.

12. Roorda, B.D., et al., DGAT1 overexpression in muscle by in vivo DNA electroporation increases intramyocellular lipid content. J Lipid Res, 2005. 46(2): p. 230-6.

13. Timmers, S., et al., Paradoxical increase in TAG and DAG content parallel the insulin sensitizing effect of unilateral DGAT1 overexpression in rat skeletal muscle. PLoS One, 2011. 6(1): p. e14503.

14. Liu, L., et al., Paradoxical coupling of triglyceride synthesis and fatty acid oxidation in skeletal muscle overexpressing DGAT1. Diabetes, 2009. 58(11): p. 2516-24.

15. Goodpaster, B.H., et al., Skeletal muscle lipid content and insulin resistance: evidence for a paradox in endurance-trained athletes. J Clin Endocrinol Metab, 2001. 86(12): p. 5755-61.

16. Amati, F., et al., Skeletal muscle triglycerides, diacylglycerols, and ceramides in insulin resistance: another paradox in endurance-trained athletes? Diabetes, 2011. 60(10): p. 2588-97.

17. Koves, T.R., et al., PPARgamma coactivator-1alpha contributes to exercise-induced regulation of intramuscular lipid droplet programming in mice and humans. J Lipid Res, 2013. 54(2): p. 522-34.

18. Dalen, K.T., et al., LSDP5 is a PAT protein specifically expressed in fatty acid oxidizing tissues. Biochim Biophys Acta, 2007. 1771(2): p. 210-27.

19. Yamaguchi, T., et al., MLDP, a novel PAT family protein localized to lipid droplets and enriched in the heart, is regulated by peroxisome proliferator-activated receptor alpha. J Biol Chem, 2006. 281(20): p. 14232-40.

20. Wolins, N.E., et al., OXPAT/PAT-1 is a PPAR-induced lipid droplet protein that promotes fatty acid utilization. Diabetes, 2006. 55(12): p. 3418-28.

21. Wang, H., et al., Perilipin 5, a lipid droplet-associated protein, provides physical and metabolic linkage to mitochondria. J Lipid Res, 2011. 52(12): p. 2159-68.

22. Bosma, M., et al., Overexpression of PLIN5 in skeletal muscle promotes oxidative gene expression and intramyocellular lipid content without compromising insulin sensitivity. Biochim Biophys Acta, 2013. 1831(4): p. 844-52.

23. Mason, R.R., et al., PLIN5 deletion remodels intracellular lipid composition and causes insulin resistance in muscle. Mol Metab, 2014. 3(6): p. 652-63.

24. Bosma, M., et al., Perilipin 2 improves insulin sensitivity in skeletal muscle despite elevated intramuscular lipid levels. Diabetes, 2012. 61(11): p. 2679-90.

25. Covington, J.D., et al., Perilipin 3 Differentially Regulates Skeletal Muscle Lipid Oxidation in Active, Sedentary and Type 2 Diabetic Males. J Clin Endocrinol Metab, 2015: p. JC20144125.

26. Mason, R.R. and M.J. Watt, Unraveling the roles of PLIN5: linking cell biology to physiology. Trends Endocrinol Metab, 2015. 26(3): p. 144-152.

27. Kershaw, E.E., et al., Adipose triglyceride lipase: function, regulation by insulin, and comparison with adiponutrin. Diabetes, 2006. 55(1): p. 148-57.

28. Fan, B., et al., High glucose, insulin and free fatty acid concentrations synergistically enhance perilipin 3 expression and lipid accumulation in macrophages. Metabolism, 2013. 62(8): p. 1168-79.

29. Hoeks, J., et al., Prolonged fasting identifies skeletal muscle mitochondrial dysfunction as consequence rather than cause of human insulin resistance. Diabetes, 2010. 59(9): p. 2117-25.

30. Chomczynski, P. and N. Sacchi, Single-step method of RNA isolation by acid guanidinium thiocyanatephenol-chloroform extraction. Anal Biochem, 1987. 162(1): p. 156-9.

31. Bustin, S.A., Absolute quantification of $m R N A$ using real-time reverse transcription polymerase chain reaction assays. J Mol Endocrinol, 2000. 25(2): p. 169-93. 
32. Sparks, L.M., et al., A high-fat diet coordinately downregulates genes required for mitochondrial oxidative phosphorylation in skeletal muscle. Diabetes, 2005. 54(7): p. 1926-33.

33. Koopman, R., G. Schaart, and M.K. Hesselink, Optimisation of oil red $O$ staining permits combination with immunofluorescence and automated quantification of lipids. Histochem Cell Biol, 2001. 116(1): p. 63-8.

34. Schneider, C.A., W.S. Rasband, and K.W. Eliceiri, NIH Image to ImageJ: 25 years of image analysis. Nat Methods, 2012. 9(7): p. 671-5.

35. Kimmel, A.R. and C. Sztalryd, Perilipin 5, a lipid droplet protein adapted to mitochondrial energy utilization. Curr Opin Lipidol, 2014. 25(2): p. 110-7.

36. Schrauwen, P., et al., Mitochondrial dysfunction and lipotoxicity. Biochim Biophys Acta, 2010. 1801(3): p. 266-71.

37. Wang, H., et al., Unique regulation of adipose triglyceride lipase (ATGL) by perilipin 5, a lipid dropletassociated protein. J Biol Chem, 2011. 286(18): p. 15707-15.

38. Granneman, J.G., et al., Functional interactions between Mldp (LSDP5) and Abhd5 in the control of intracellular lipid accumulation. J Biol Chem, 2009. 284(5): p. 3049-57.

39. Billecke, N., et al., Perilipin 5 mediated lipid droplet remodelling revealed by coherent Raman imaging. Integr Biol (Camb), 2015.

40. Bosma, M., et al., The lipid droplet coat protein perilipin 5 also localizes to muscle mitochondria. Histochem Cell Biol, 2012. 137(2): p. 205-16.

41. Wang, C., et al., Perilipin 5 improves hepatic lipotoxicity by inhibiting lipolysis. Hepatology, 2015. 61(3): p. 870-82.

42. Kiens, B., Skeletal muscle lipid metabolism in exercise and insulin resistance. Physiol Rev, 2006. 86(1): p. 205-43.

43. Hoeg, L.D., et al., Lipid-induced insulin resistance affects women less than men and is not accompanied by inflammation or impaired proximal insulin signaling. Diabetes, 2011. 60(1): p. 64-73.

44. Lafontan, M. and D. Langin, Lipolysis and lipid mobilization in human adipose tissue. Prog Lipid Res, 2009. 48(5): p. 275-97.

45. Peters, S.J., et al., Perilipin family (PLIN) proteins in human skeletal muscle: the effect of sex, obesity, and endurance training. Appl Physiol Nutr Metab, 2012. 37(4): p. 724-35. 
CHAPTER 4

Supplemental Table 1: Primer sequences

\begin{tabular}{llll}
\hline Gene & Forward & Reverse & Probe \\
\hline PLIN5 & GAGCCATGCTGTGGATGTTGTA & CAGTGCCGCGAGCTCTTC & TGGATCACTTCCTGCCCATGACGG \\
RPLP0 & CCATTCTATCATCAACGGGTACAA & AGCAAGTGGGAAGGTGTAATCC & \\
\hline
\end{tabular}




\title{
CHAPTER 5
}

\section{Dissociation of intramyocellular lipid storage and insulin resistance in trained athletes and patients with type 2 diabetes; involvement of the lipid droplet coat protein Perilipin 5}

\author{
Anne Gemmink*, Sabine Daemen *, Bram Brouwers, Peter R Huntjens, Gert \\ Schaart, Joris Hoeks, Patrick Schrauwen, Matthijs K.C. Hesselink
}

In preparation

*These authors contributed equally to this work 


\section{ABSTRACT}

Excess storage of intramyocellular lipids (IMCL) is considered to hamper insulin sensitivity. However, this is not observed in endurance-trained athletes. Trained have high levels of Perilipin 5 (PLIN5) protein content compared to type 2 diabetes patients (T2DM). Skeletal muscle PLIN5 overexpression prevents insulin resistance despite an increase in IMCL content. In addition, an increase in PLIN5 coated lipid droplets (LDs) blunted fasting-induced insulin resistance. Here, we tested the hypothesis that the fraction of PLIN5 coated LDs is a determinant of skeletal muscle insulin sensitivity.

Muscle biopsies were taken from eight trained $\left(\mathrm{VO}_{2 \max }>55 \mathrm{ml} / \mathrm{kg} / \mathrm{min}\right)$, lean sedentary $\left(\mathrm{VO}_{2 \max }<45 \mathrm{ml} / \mathrm{kg} / \mathrm{min}\right)$, obese and T2DM subjects. Trained, Obese and T2DM were matched for total IMCL content. Sections were stained for Bodipy 493/503, PLIN5 and laminin. Images were acquired on a Leica TCS SPE confocal microscope and analyzed for lipid area fraction, LD size and number. LDs were separated in PLIN5+ and PLIN5- LDs. A stepwise multiple linear regression was performed to determine which factors explain the variability in insulin sensitivity.

Trained and T2DM differed in lipid storage pattern, a higher LD number in Trained $\left(0.037 \pm 0.004 \mu \mathrm{m}^{-}\right.$

2 vs. $\left.0.023 \pm 0.003 \mu \mathrm{m}^{-2}, p=0.024\right)$ and larger LDs in T2DM $\left(0.27 \pm 0.01 \mu \mathrm{m}^{2}\right.$ vs. $0.32 \pm 0.02 \mu \mathrm{m}^{2}$, $p=0.197)$, though not significant. Despite differences in PLIN5 protein content $(1.65 \pm 0.21 \mathrm{AU}$ and $0.89 \pm 0.09 \mathrm{AU}$ for Trained and T2DM, $p=0.004$ ) lipid storage pattern of PLIN5+ and PLIN5- LDs was similar as total IMCL. None of the LD parameters and PLIN5 protein content correlated with insulin sensitivity. The strongest predictor for insulin sensitivity was $\mathrm{VO}_{2 \max }$. PLIN5 protein content and PLIN5+ LD number only contributed to the prediction of insulin sensitivity when $\mathrm{VO}_{2 \max }$ was included in the model.

The putative relation between PLIN5 and insulin sensitivity appears to be indirect and may require a high oxidative capacity. Thus, PLIN5 abundance cannot explain in a direct way the athlete's paradox. 


\section{INTRODUCTION}

Excess storage of fat in non-adipose tissue like skeletal muscle is considered to hamper insulin sensitivity. Despite the frequently reported negative association between intramyocellular fat content (IMCL) and insulin sensitivity in insulin resistant subjects [1-3], no such association has been reported for trained athletes, who store myocellular fat abundantly, whilst maintaining insulin sensitivity, a phenomenon referred to as 'the athletes' paradox' [4]. In line with these cross-sectional observations, experimentally augmenting fat storage by lipid infusion renders skeletal muscle insulin resistance in healthy lean subjects, whereas in trained athletes, who can readily shuttle the infused lipids towards oxidation or storage in myocellular LDs, this effect is alleviated [5]. Lipidomic studies revealed that composition of the lipid droplets (LD) in skeletal muscle biopsies of master athletes differs from the composition of myocellular lipid droplets in age matched patients with type 2 diabetes [6], an observation paralleled by increased protein content of the lipid droplet coat protein PLIN5 in the biopsies of the trained master athletes compared to the age matched patients with type 2 diabetes.

PLIN5 is a member of the perilipin lipid droplet coat proteins with a putative role in liberating fatty acids for oxidative degradation in mitochondria [7, 8]. Using an in vivo approach, we recently showed in rat skeletal muscle that gene electroporation-mediated overexpression of PLIN5 promoted high-fat diet-induced steatosis in muscle, predominantly originating from an increase in LD size. Despite the increased fat content in PLIN5 overexpressing muscle, PLIN5 overexpressing muscle maintained its insulin sensitivity [9]. In the same model we noticed LD composition of LDs coated with PLIN5 differs from LDs devoid of PLIN5 [10]. These data indicate that abundance of PLIN5 on myocellular LDs may promote benign (rather than insulin desensitizing) handling of myocellular fat.

In a human model of physiological insulin resistance (prolonged fasting) we noted that those whose muscle fat content increased most upon fasting, were the subjects who dropped least in insulin sensitivity. Similarly, subjects capable of storing myocellular fat abundantly were those whose mitochondrial respiratory capacity (on a variety of substrates) was least compromised [11]. Given these protective properties of PLIN5, differences in PLIN5 content may be an underlying factor helping to understand the 'athlete's paradox'. Using our custom developed approach to distinctly image lipid droplets coated with PLIN5 (PLIN5+ LDs) and those devoid of PLIN5 (PLIN5- LDs), we showed that the previously observed protective effect of having a high capacity to expand muscle fat content, originated from PLIN5+ LDs. No such protective effects were made for LDs devoid of PLIN5. Thus, coating of LDs with PLIN5 in skeletal muscle may promote benign storage of fat in LDs, 
at least under conditions of physiological insulin resistance. In the present study we test the hypothesis that, in the face of similar IMCL content, the fraction of PLIN5+ LDs is a determinant of skeletal muscle insulin sensitivity and related parameters and may help to explain the athlete's paradox. To this end we examined subjects with comparable IMCL content but at both extremes of the spectrum of insulin sensitivity. Thus, we selected healthy, lean, insulin sensitive athletes and their BMI- and age matched non-athletic controls, along with obese, aged, insulin resistant type 2 diabetes patients and their BMI and age matched normoglycemic controls. Trained athletes, obese participants and patients with type 2 diabetes were purposely selected to have similar IMCL content.

\section{METHODS}

\section{Subjects}

Eight young endurance trained subjects $\left(\mathrm{VO}_{2 \max }>55 \mathrm{ml} / \mathrm{kg} / \mathrm{min}\right)$ (Trained), lean sedentary subjects $\left(\mathrm{VO}_{2 \max }<45 \mathrm{ml} / \mathrm{kg} / \mathrm{min}\right)$ (Lean), middle-aged obese subjects (Obese) and type 2 diabetes patients (T2DM) participated in this study. All subjects gave written informed consent before participating in this study. These subjects were selected from a larger cohort based for having similar IMCL content in the Trained, the Obese and T2DM. As we and others previously observed that IMCL content in lean sedentary subjects is consistently lower than IMCL in trained, obese and patients with type 2 diabetes, lean subjects were randomly selected from the larger cohort.

All subjects underwent a hyperinsulinemic-euglycemic clamp with a muscle biopsy taken from the $m$. vastus lateralis. A part of the muscle biopsy was directly frozen in melting isopentane and stored at $-80^{\circ} \mathrm{C}$ for histochemical and western blot analysis. Another portion was placed in ice-cold modified SET-buffer (250nM Sucrose, 10mM Tris-HCl, $1 \mathrm{mM}$ EDTA and $2 \mathrm{mM}$ ATP) to measure ex vivo

${ }^{14} \mathrm{C}$ palmitate oxidation. Maximal oxidative capacity was measured with a graded maximal cycle test. Oxygen consumption was measured throughout the test (Omnical, Maastricht, The Netherlands). Furthermore, body composition was measured with a DEXA scan and fasting plasma glucose was determined.

\section{Histochemical analysis}

For immunofluorescence analysis of IMCL content, LD size, LD number and PLIN5 $7 \mu \mathrm{m}$ thick cryosections were cut and mounted on glass slides. Sections were stained as described previously 
[11] In short, fixation was performed with $3.7 \%$ formaldehyde for 30 minutes and blocked for 45 minutes with blocking buffer $(150 \mathrm{mM} \mathrm{NaCl}, 20 \mathrm{mM}$ Tris pH 6.8 and $2 \% \mathrm{BSA})$. Subsequently, cryosections were incubated with primary antibodies against laminin (L9393, Sigma, St. Louis, USA), myosin heavy chain type I (A4.840, Developmental Studies Hybridoma Bank, lowa City, IA, USA) and PLIN5 (GP31, Progen Biotechnik, Heidelberg, Germany) for 1 hour. Thereafter, sections were incubated with Bodipy 493/503 (D3922, Molecular Probes, Leiden, The Netherlands) and appropriate secondary antibodies conjungated with AlexaFluor-405, AlexaFluor-555 and AlexaFluor647. Sections were mounted in Mowiol and stored in the dark until imaging (typically within 7 days after staining).

To assess muscle fiber type distribution and quantify IMCL content (Bodipy 493/503) for subject selection, images were taken using a Nikon E800 fluorescence microscope (Ulvikon, Bunnik, The Netherlands) coupled to a Nikon DS-Fi1c color CCD camera (Nikon, Amsterdam, The Netherlands). The relative fraction of type I and type II muscle fibers was individually determined using ImageJ [12]. Subsequent detailed analysis of LD area fraction, LD number and LD size was performed according to individual fiber type distribution using a Leica TCS SPE confocal microscope with 405, 488 and 647 nm laser lines for respectively Laminin-AF405, Bodipy 493/503 and PLIN5-AF647. A 63x 1.3 N.A. oil immersion objective and 1.1x optical zoom at 2048×2048 pixels was used resulting in a pixel size of $77 \mathrm{~nm}$. Subsequently, the images were deconvoluted using Huygens Essential software (Scientific Volume Imaging B.V., Hilversum, The Netherlands) to remove background and to improve signal-to-noise ratio. Images were analyzed for LD size and number with ImageJ. Subsequently LDs were subdivided into PLIN5 positive LDs (PLIN5+) and LDs devoid of PLIN5 (PLIN5-) with Matlab R2012a (The Mathworks, Inc., Natick, Massachusetts, USA).

\section{Western Blotting}

For western blot analysis an antibody against PLIN5 (GP31, Progen Biotechnik, Heidelberg, Germany) was used. Infra-red tagged secondary antibody (IRDye, LI-COR, Lincoln, NE, USA) was used to visualize and quantify the appropriate band (Odyssey Infrared Imaging system, LI-COR Biosciences, Westburg, Leusden, The Netherlands). 


\section{${ }^{14} \mathrm{C}$ palmitate oxidation assay}

Fresh muscle biopsy material in ice-cold SET buffer was homogenized and $80 \mu \mathrm{l}$ was loaded in a modified 48-wells plate in triplicate. Reactions were initiated with the addition of working buffer (0.2 mM palmitate and $1 \mu \mathrm{Ci} / \mathrm{mL}\left[1-{ }^{14} \mathrm{C}\right]$-palmitate (NEC075H250UC, Perkin Elmer, Waltham, MA, USA), 7.5\% fatty acid free BSA $100 \mathrm{mM} \mathrm{KCl}, 62.5 \mathrm{mM}$ Sucrose, $12.5 \mathrm{mM} \mathrm{KH}_{2} \mathrm{PO}_{4}, 10 \mathrm{mM}$ Tris- $\mathrm{HCl}, 2$ mM ATP, 1.25 mM MgCl, 1.25 mM L-Carnitine, 1.25 mM DTT, 0.125 mM Malic Acid, 0.125 mM NAD ${ }^{+}$ and $0.0625 \mathrm{mM}$ Coenzyme A). The plate was incubated for 2 hours at $37^{\circ} \mathrm{C}$. Reactions were terminated with the addition of $40 \mu \mathrm{l} 70 \%$ perchloric acid. The plate was transferred to a shaking table and incubated for 1 hour. The released $\mathrm{CO}_{2}$ was trapped in $1 \mathrm{M} \mathrm{NaOH}$ in the adjacent well. Subsequently $200 \mu \mathrm{l} \mathrm{NaOH}$ containing the trapped $\mathrm{CO}_{2}$ was transferred to a scintillation vial with OptiFluor scintillation fluid. Radioactivity was counted on a Tri-Carb 2910 TR Liquid Scintillation Analyzer (Perkin Elmer, Waltham, MA, USA).

\section{Statistics}

Results are presented as mean \pm SEM. Statistical analysis were performed using SPSS version 21.0 (SPSS, Chicago, IL, USA). A One-Way ANOVA with a Bonferroni post-hoc test was used to test for statistical differences between groups for total IMCL content, LD area fraction, LD size and LD number of all LDs, and PLIN5 protein content. Upon distinction between PLIN5+ and PLIN5- LDs, statistical differences between groups was tested with a Repeated Measures ANOVA with PLIN5 (PLIN5+ vs. PLIN5- LDs) as a within subject factor. A One-way ANOVA was used when there was a significant interaction effect between PLIN5 and group to test for statistical differences between groups for PLIN5+ and PLIN5- LDs separately. A Bonferroni post-hoc test was performed to specify which groups statistically differed. A paired samples t-test was used to test for each group significant differences between PLIN5+ and PLIN5- LDs when there was a significant interaction effect and was corrected for multiple comparisons (4 groups) with Bonferroni. Pearson's correlation coefficients were used to test for significant linear association between variables. We investigated the influence of oxidative capacity (measured by $\mathrm{VO}_{2 \mathrm{Max}}$ ) and PLIN5 content on insulin sensitivity (glucose infusion rate, GIR) by stepwise multiple linear regression analysis. $p<0.05$ was considered to be significant. 


\section{RESULTS}

\section{Subject characteristics}

Subject characteristics are shown in Table 1. Obese and T2DM were significantly older than Trained and Lean. Fat mass was the highest in the Obese and T2DM and the lowest in Trained. By design, patients with type 2 diabetes had higher fasting plasma glucose levels and lower GIR (Figure 1A) compared to the other groups. $\mathrm{VO}_{2 \max }$ and fatty acid oxidative capacity were highest in Trained and the lowest in T2DM.

Table 1. Subject characteristics

\begin{tabular}{lllll}
\hline Parameter & Athletes & Untrained & Obese & T2DM \\
\hline Age (years) & $26.0 \pm 1.8$ & $23.5 \pm 1.2$ & $54.1 \pm 3.1^{*+}$ & $60.6 \pm 2.0^{*+}$ \\
Body weight $(\mathrm{kg})$ & $72.4 \pm 2.6$ & $73.1 \pm 2.3$ & $96.1 \pm 3.3^{*+}$ & $95.9 \pm 2.8^{*+}$ \\
$\%$ Fat mass & $13.4 \pm 0.5$ & $18.4 \pm 1.5^{*}$ & $28.9 \pm 0.9^{*+}$ & $28.3 \pm 0.9^{*+}$ \\
$\mathrm{BMI}\left(\mathrm{kg} / \mathrm{m}^{2}\right)$ & $21.0 \pm 0.6$ & $22.2 \pm 0.6$ & $29.4 \pm 0.4^{*+}$ & $29.6 \pm 0.8^{*+}$ \\
Fasting glucose $(\mathrm{mmol} / \mathrm{L})$ & $5.14 \pm 0.09$ & $5.24 \pm 0.10$ & $4.91 \pm 0.28$ & $7.43 \pm 0.53^{*+\neq}$ \\
$\mathrm{VO}_{2 \max }(\mathrm{ml} \mathrm{O} / \mathrm{kg}$ lean mass $/ \mathrm{min})$ & $71.0 \pm 1.62$ & $51.8 \pm 1.70^{*}$ & $39.8 \pm 1.93^{*+}$ & $36.8 \pm 1.45^{*+}$ \\
$14 \mathrm{C}$ palmitate oxidation $(\mathrm{nmol} / 2 \mathrm{~h} / \mathrm{mg})$ & $0.22 \pm 0.03$ & $0.13 \pm 0.02^{*}$ & $0.08 \pm 0.02^{*}$ & $0.08 \pm 0.02^{*}$ \\
\hline
\end{tabular}

${ }^{*} p<0.05$ vs. Trained; $\uparrow p<0.05$ vs. Lean; $\ddagger p<0.05$ vs. Obese

\section{Muscle fat content}

We successfully selected three groups with similar IMCL content (trained lean subjects, obese normoglycemic subjects and patients with type 2 diabetes, Figure 1B) for detailed LD analysis. Examination of fiber type distribution in sections of these subjects ( $123 \pm 12$ cells) revealed that the fraction of type I muscle fibers was higher in the Trained group than in any of the other groups, albeit non-significantly (59\% vs. $42 \%, 43 \%$ and $45 \%$ for Trained, Lean; $p=0.058$ vs. Trained, Obese; $p=0.086$ vs. Trained and T2DM; $p=0.192$ vs. Trained, respectively). 
A

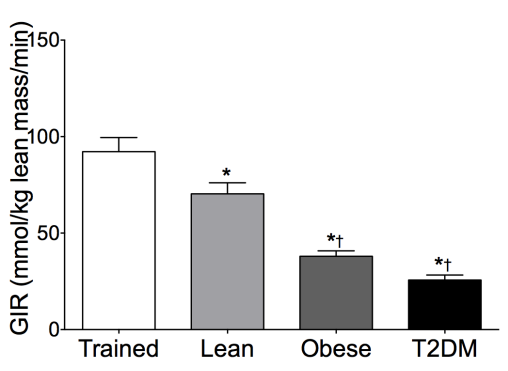

C

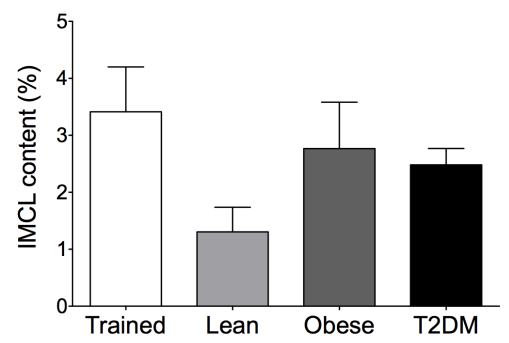

B

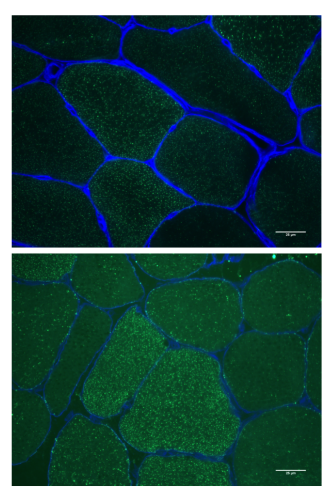

Figure 1. (A) Insulin sensitivity of Trained, Lean, Obese and T2DM patients. (B) Representative images of IMCL content in Trained, Obese and T2DM. (C) Groups were matched for total IMCL content. ${ }^{*} p<0.05$ vs. Trained and $+p<0.05$ vs. Lean.

Detailed LD analysis was performed using confocal microscopy in a selection of muscle cells representing the fiber type distribution of the individual. This revealed that, as targeted, the lipid area fraction in T2DM was not significantly different from the lipid area fraction in Trained $(0.95 \pm 0.07 \%, 0.73 \pm 0.13$ for Trained and T2DM respectively; $p=0.818$ Figure $2 \mathrm{~A})$. Upon inclusion of the Lean and the Obese group, we observed that maximal aerobic capacity $\left(\mathrm{VO}_{2} \mathrm{max}\right)$ correlated positively with lipid area fraction ( $r=0.530 ; p=0.003$, Figure $2 \mathrm{~B}$ ). Lipid area fraction is the product of LD number and LD size, relative to muscle fiber area. Lipid droplet size and number may be differentially affected by training status [13] and has been differentially linked to insulin resistance [11]. Although the total lipid area fraction was similar in Trained and T2DM, this was accomplished differently: Trained had significantly more LDs than T2DM $\left(0.037 \pm 0.004 \mu \mathrm{m}^{-2}\right.$ vs. $0.023 \pm 0.003 \mu \mathrm{m}^{-2}$ for Trained and T2DM, respectively; $p=0.024$, Figure $2 \mathrm{C}$ ). Whereas in T2DM the high lipid area fraction resulted from modestly more LDs than in Lean and Obese $\left(0.020 \pm 0.004 \mathrm{\mu m}^{-2}\right.$ and $0.017 \pm 0.002 \mu \mathrm{m}^{-2}$ for respectively Lean and Obese; $p>0.05$ vs. T2DM), but predominantly resulted from larger LDs $\left(0.27 \pm 0.01 \mu \mathrm{m}^{2}, 0.26 \pm 0.02 \mu \mathrm{m}^{2}, 0.26 \pm 0.02 \mu \mathrm{m}^{2}\right.$ and $0.32 \pm 0.03 \mu \mathrm{m}^{2}$ for respectively Trained, Lean, Obese and T2DM; Figure 2D; $p=0.197)$. The tight correlation between $\mathrm{VO}_{2} \max$ and LD number $\left(r=0.659 ; p<0.001\right.$, Figure $2 \mathrm{E}$ ) and the lack of such a correlation between $\mathrm{VO}_{2}$ max and LD size $(r=-0.185 ; p=0.336$, Figure $2 \mathrm{~F})$ suggests that - within these groups- maximal oxidative capacity is a determinant of LD number rather than LD size. 
A

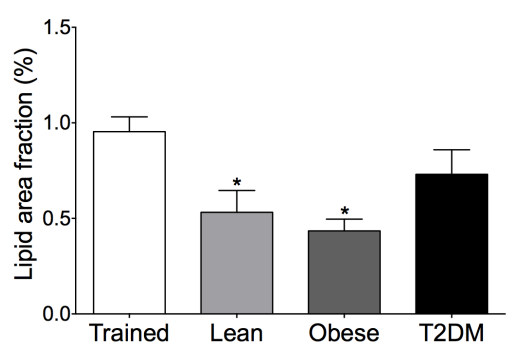

D

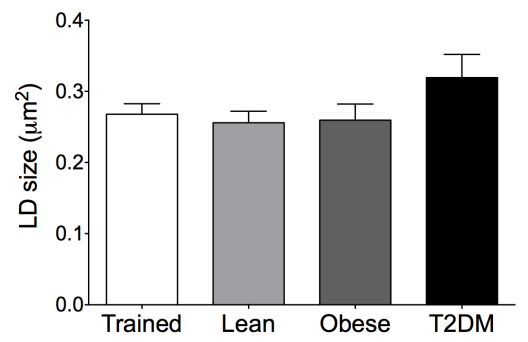

B

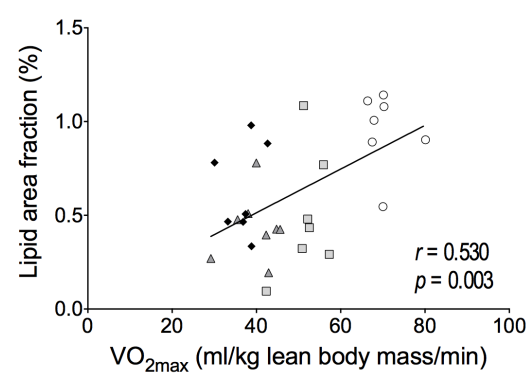

E

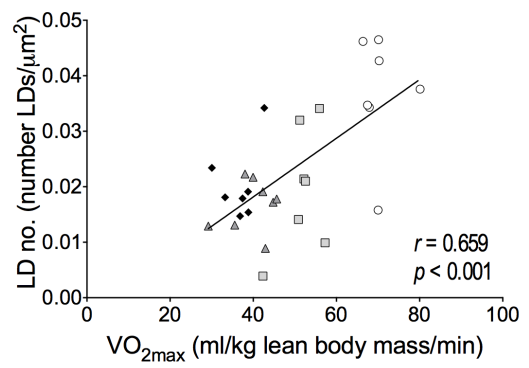

C

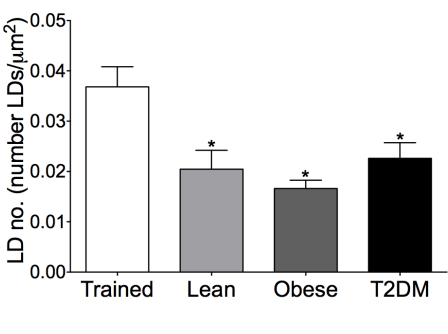

$\mathbf{F}$

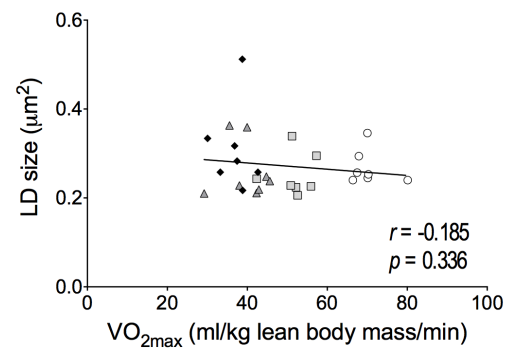

Figure 2. (A) Lipid area fraction measured by confocal microscopy. (B) $\mathrm{VO}_{2 \max }$ correlates with lipid area fraction. (C) Quantification of number of lipid droplets and (D) lipid droplet size. Correlations between $\mathrm{VO}_{2 \max }$ and (E) lipid droplet number (F), and lipid droplet size; circles: Trained, squares: Lean, triangles: Obese and diamonds: T2DM. ${ }^{*} p<0.05$ vs. Trained.

\section{PLIN5 associated muscle fat content and insulin sensitivity}

A high oxidative capacity has been reported to associate with high levels of PLIN5 protein content [6]. Thus, we explored if the differences in lipid area fraction was accounted for by PLIN5 coated LDs and if this was related to maximal oxidative capacity. Western blot analysis revealed that PLIN5 protein content was significantly higher in trained athletes compared to any of the other groups (Figure 3B). Our novel microscopy approach [11] permits making the distinction between PLIN5+ LDs and PLIN5- LDs. Upon making this distinction, lipid area faction of both the PLIN5+ as well as the PLIN5- LDs (Figure 3C) mimicked the pattern observed for lipid area fraction of all LDs (Figure 2A). PLIN5+ lipid area fraction in Trained was not significantly different from PLIN5+ lipid area fraction in T2DM $(0.79 \pm 0.07 \%$ and $0.53 \pm 0.10 \%$ for Trained and T2DM respectively $p=0.230$ ), but was higher than Lean and Obese $(0.43 \pm 0.11 \%$ and $0.34 \pm 0.04 \%$ for respectively Lean and Obese, $p<0.05$ vs. Trained). The PLIN5+ lipid area fraction, however, was higher than the PLIN5- lipid area fraction in all groups (Figure $3 \mathrm{C}$ ) and appeared to originate predominantly from more as well larger LDs (Figure 3D 
and 3E) in the PLIN5+ LDs. The pattern observed for LD area fraction, number or size, upon making the distinction between PLIN5+ and PLIN5- LDs, however, did not yield different results between the groups than when simply examining all LDs irrespective of their coating with PLIN5. It should be noted though, that the fraction of PLIN5+ LDs (relative to the total number of LDs), was somewhat (albeit non-significantly $p=0.182$ ) lower in T2DM than in any of the other groups $(0.77 \pm 0.03$, $0.73 \pm 0.05,0.74 \pm 0.05$ and $0.64 \pm 0.04$ for respectively Trained, Lean, Obese and T2DM, Figure 3F).

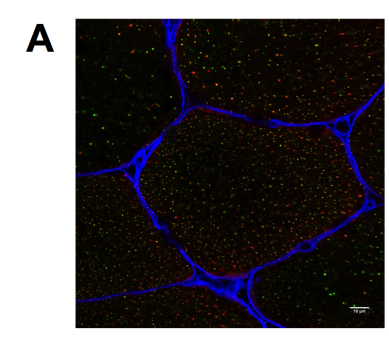

B

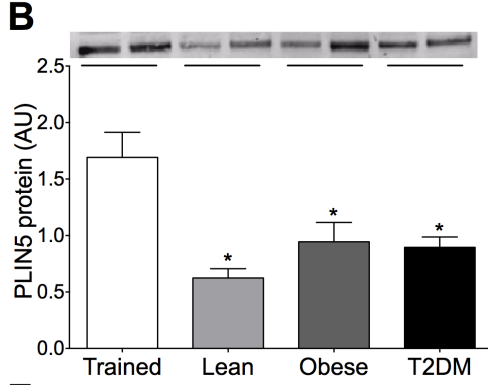

E

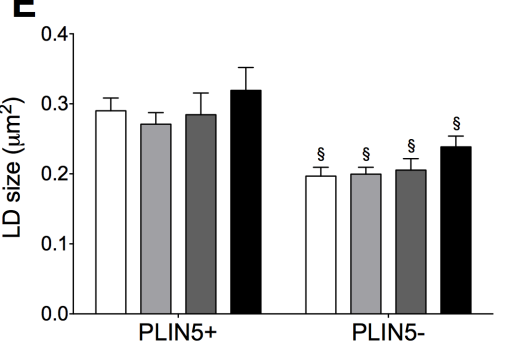

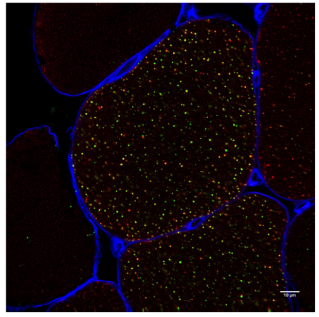

C

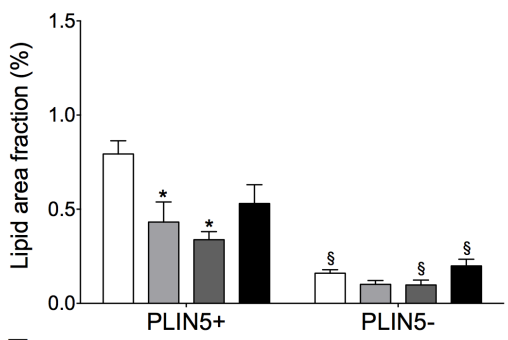

$\mathbf{F}$

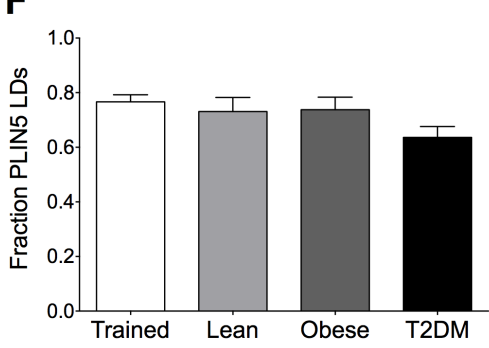

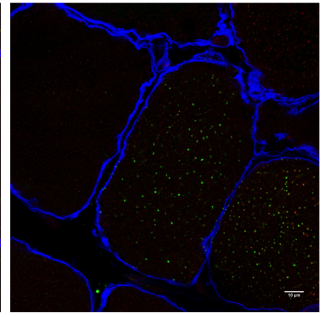

D

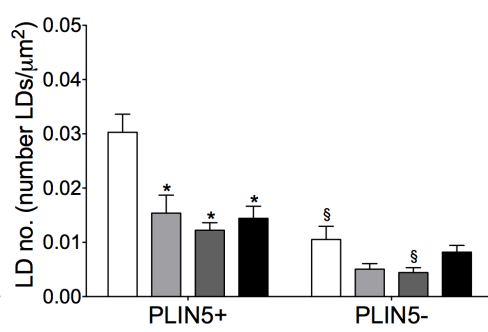

PLIN5-

Figure 3. (A) Representative confocal images of each group. Cell membranes are stained in blue, PLIN5 in red and lipid droplets in green. (B) Whole muscle lysates PLIN5 protein content. (C) Lipid area fraction, (D) size and (E) number of lipid droplets coated with and devoid of PLIN5. (F) Fraction of lipid droplets coated with PLIN5 from the total lipid droplet pool. * $p<0.05$ vs. Trained and $\S p<0.05$ vs. PLIN5+.

In rodent models $[9,14]$ as well as in human fasting-mediated lipid-related insulin resistance [11], a protective role for PLIN5 in maintaining peripheral insulin sensitivity has been shown. Thus, we explored if lipid droplet morphology and the coating of the LDs with PLIN5 was related with peripheral insulin sensitivity in subjects with a wide range in insulin sensitivity, including highly insulin sensitive trained subjects with abundance of PLIN5 and insulin resistant T2DM with low PLIN5 
levels. We did not observe a significant correlation between PLIN5 content as assessed by Western blotting and glucose infusion rate $(r=0.259, p=0.166$; Figure $4 A)$, nor did we observe consistent significant correlations between markers of muscle lipid content (lipid area fraction, LD number or LD size) upon making the distinction between PLIN5+ and PLIN5- LDs and GIR (Figure 4B-G), suggesting that in these groups coating of the LD with PLIN5 per se, does not correlate with insulin sensitivity.
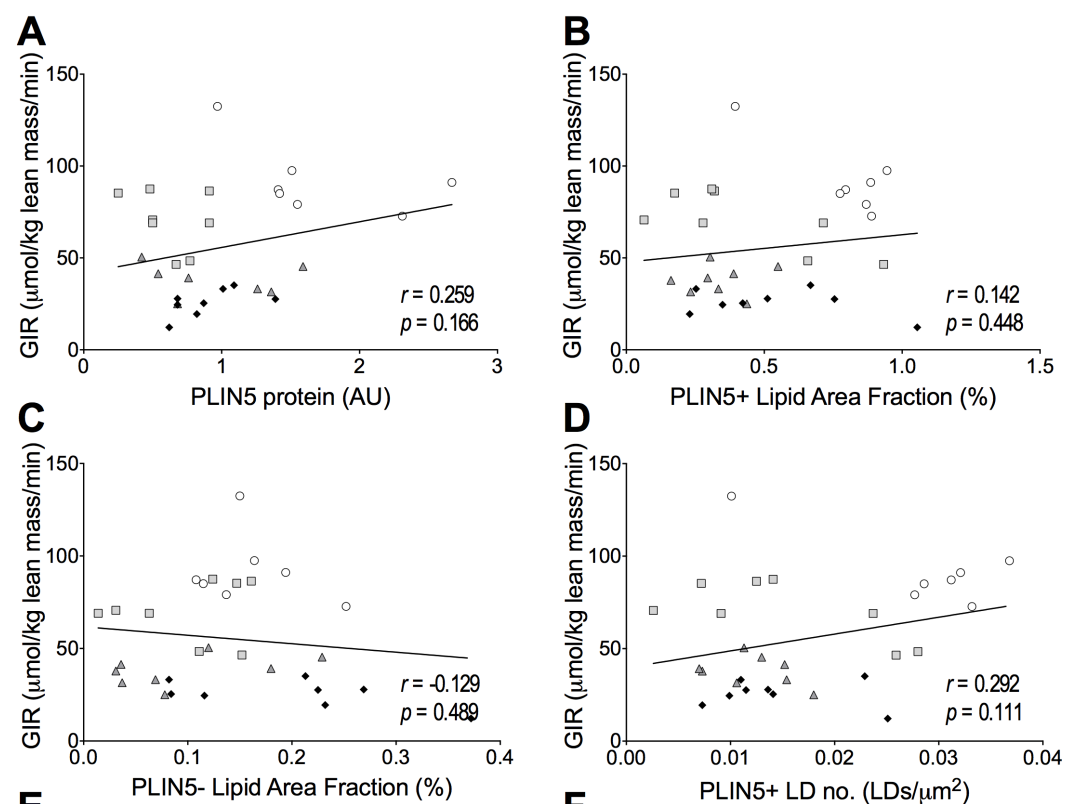

Figure 4. Correlations between insulin sensitivity and (A) PLIN5 protein content measured by Western Blotting, (B) PLIN5+ lipid area fraction, (C) PLIN5lipid area fraction, (D) PLIN5+ LD number, (E) PLIN5- LD number, (F) PLIN5+ LD size and (G) PLIN5LD size; circles: Trained, squares: Lean, triangles: Obese and diamonds: T2DM.
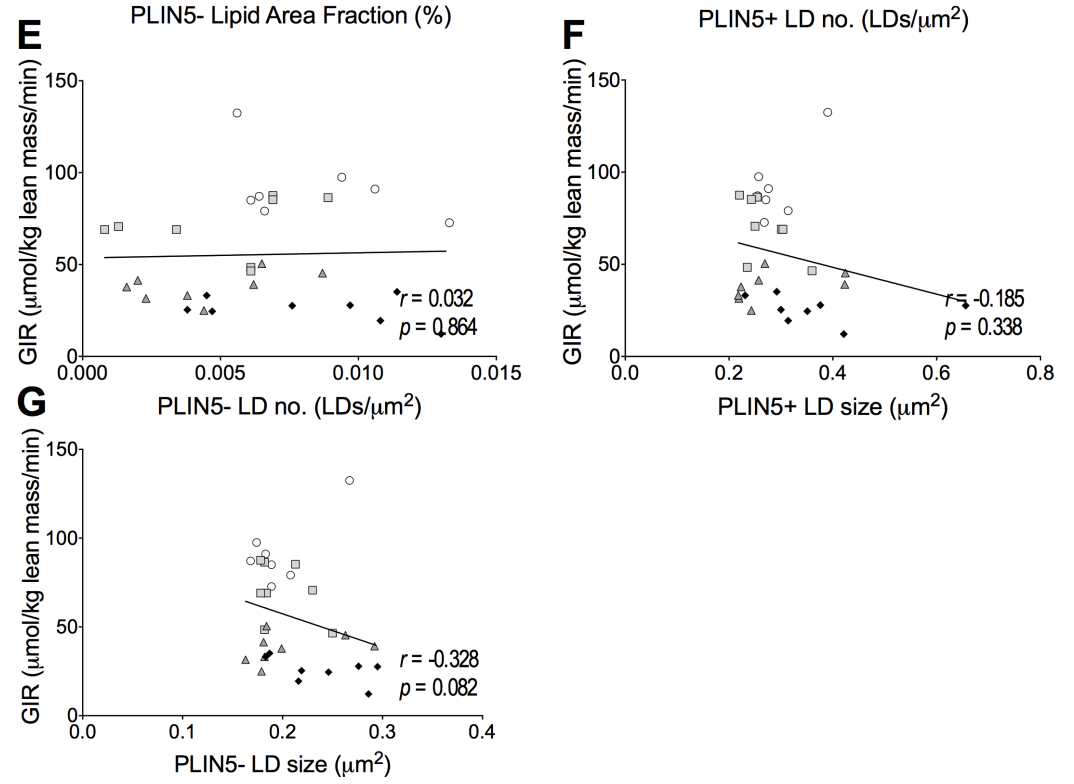


\section{PLIN5 coated LDs and oxidative capacity}

PLIN5 protein content as assessed by Western blotting correlated positively with $\mathrm{VO}_{2} \max (r=0.520$; $p=0.005$, Figure $5 \mathrm{~A}$ ). The correlations between $\mathrm{VO}_{2} \mathrm{max}$ and lipid area fraction (Figure $2 \mathrm{~B}$ ) and $\mathrm{LD}$ number (Figure 2E) appeared to be driven by PLIN5+ LDs ( $r=0.600 ; p=0.001$ for $\mathrm{VO}_{2} \max$ and PLIN5+ lipid area fraction and $r=0.695$ Figure 5B; $p<0.001$ for $\mathrm{VO}_{2}$ max and PLIN5+ LD number Figure 5C) and not by PLIN5- LDs (Figure 5D and 5E).

A

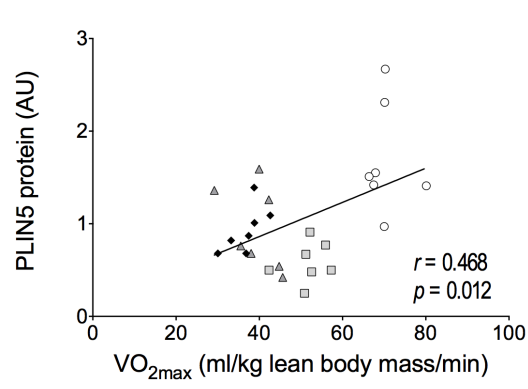

D

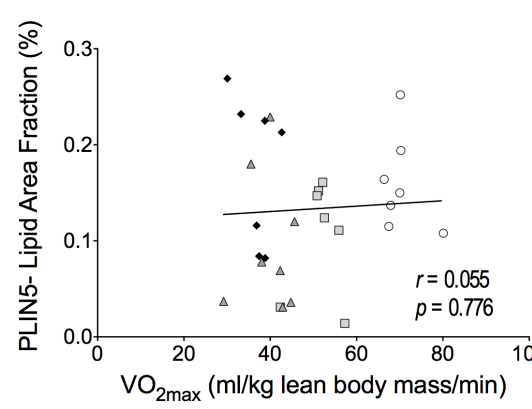

B

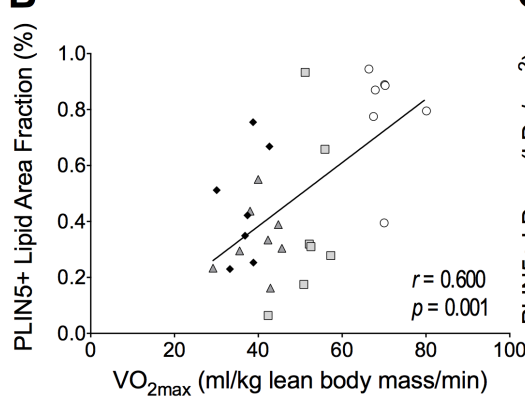

E

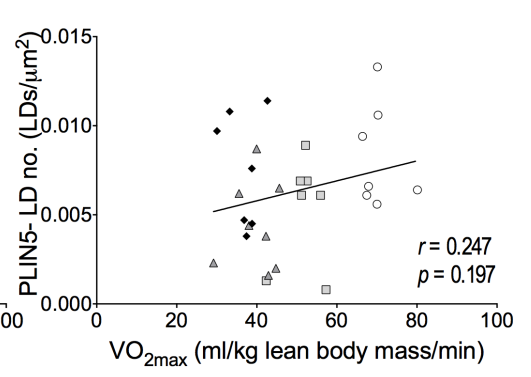

C
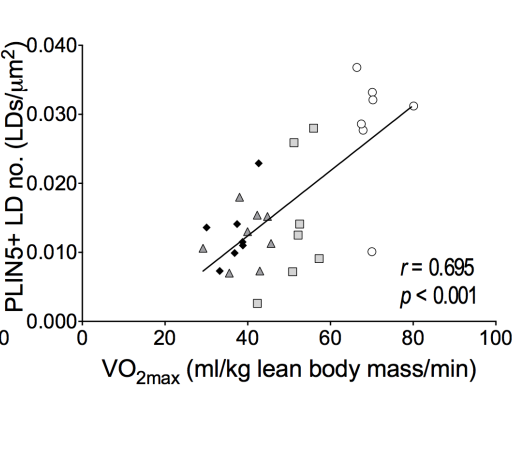

Figure 5. Correlations between $\mathrm{VO}_{2 \max }$ and (A) PLIN5 protein content, (B) PLIN5+ lipid area fraction, (C) PLIN5+ LD number, (D) PLIN5- lipid area fraction and (E) PLIN5- LD number; circles: Trained, squares: Lean, triangles: Obese and diamonds: T2DM.

The observation that correlations between maximal oxidative capacity and markers of muscle lipid fat content somehow relate to PLIN5+ (and not PLIN5-) LDs is interesting in light of previous observations that PLIN5 protein content is high in tissues with a high oxidative capacity [15] and in the trained state [16]. In addition, rodent studies seem to indicate that PLIN5 serves to fuel oxidative lipolysis [7, 8], partly by promoting oxidative gene expression [9]. Thus, we examined the involvement of PLIN5 coating with the ability to oxidize palmitate. Total lipid area fraction and number of LDs associated positively with the capacity to oxidize palmitate $(r=0.385 ; p=0.033$; Figure $6 \mathrm{~A}$ and $r=0.526 ; p=0.002$; Figure $6 \mathrm{~B}$ ). For total lipid area faction, this association was only present for 
PLIN5+ and not PLIN5- LDs ( $r=0.390 ; p=0.030$ and $r=0.227 ; p=0.219$, respectively, Figure 6C and 6D) whereas the correlation between LD number and ${ }^{14} \mathrm{CO}_{2}$ oxidation was present in PLIN5+ and PLIN5LDs $(r=0.491 ; p=0.005$ and $r=0.389 ; p=0.032$, respectively, Figure 6E and $6 \mathrm{~F})$. LD size did not correlate with the capacity to oxidize palmitate (data not shown). In addition, total PLIN5 protein content, as assessed by Western blotting, was associated with the capacity to oxidize palmitate $(r=0.362$; $p<0.05$; Figure 6G).

A

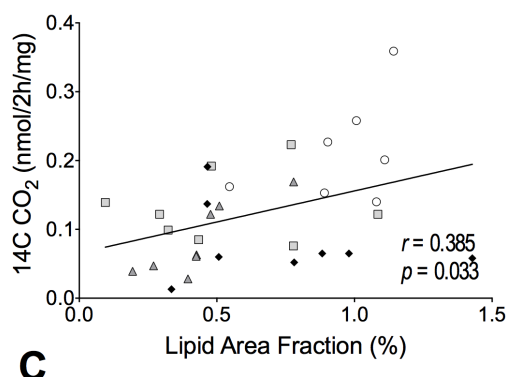

C

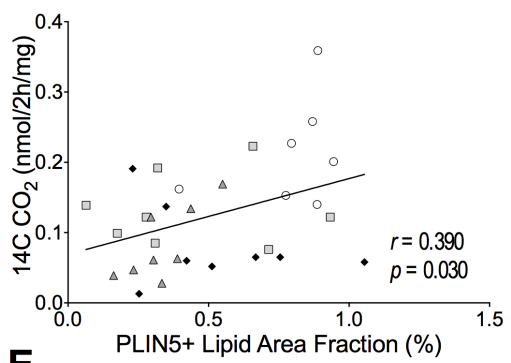

E
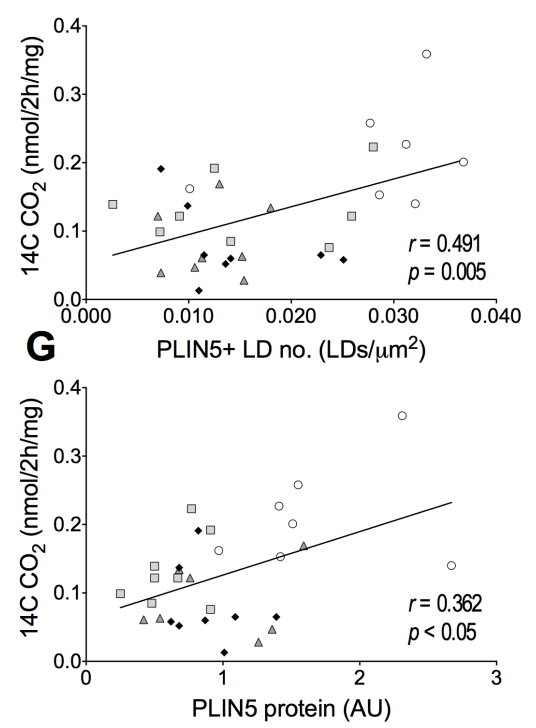

B
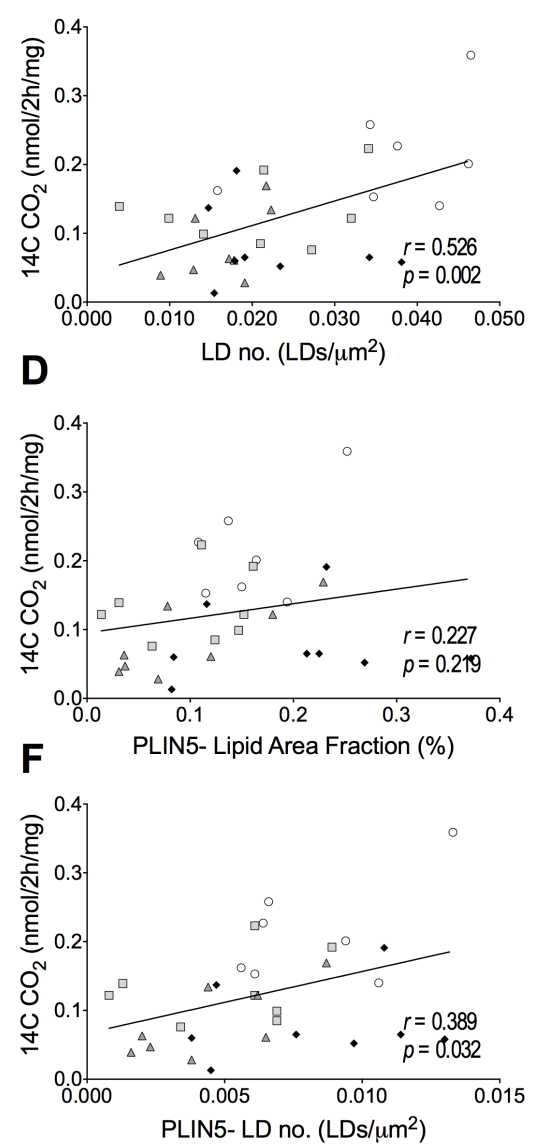

Figure 6. Correlations between capacity to oxidize fatty acids and (A) lipid area fraction, (B) LD number (C) PLIN5+ lipid area fraction, (D) PLIN5- lipid area fraction, (E) PLIN5+ LD number, (F) PLIN5- LD number and (G) PLIN5 protein content measured by Western Blotting; circles: Trained, squares: Lean, triangles: Obese and diamonds: T2DM. 


\section{Stepwise multiple regression analysis}

Stepwise linear regression analysis with glucose infusion rate (GIR) as dependent variable (Table 2) revealed that $\mathrm{VO}_{2 \max }$ was the only (and strongest) predictor of GIR in a model with $\mathrm{VO}_{2 \max }{ }^{14} \mathrm{C}$ palmitate oxidation and size of PLIN5+ and PLIN5- LDs as input parameters $\left(R^{2}=0.702 ; p<0.001\right)$. In a model in which $\mathrm{VO}_{2 \max }{ }^{14} \mathrm{C}$ palmitate oxidation and PLIN5 content based upon Westerns blotting were used as input parameters, $\mathrm{VO}_{2 \max }$ and PLIN5 content jointly explained $74.7 \%$ of the observed variance in $\mathrm{GIR}\left(\mathrm{R}^{2}=0.747 ; p<0.001\right)$. Upon making the distinction between PLIN5+ and PLIN5- LDs, it appeared that -next to $\mathrm{VO}_{2 \max }$ also the number of PLIN5+ LDs contributed significantly to the prediction of $\mathrm{GIR}\left(\mathrm{R}^{2}=0.807 ; p<0.001\right)$. This suggests that a positive effect of PLIN5 on insulin sensitivity predominantly emerges in conjunction with a high oxidative capacity.

Table 2. Parameters predicting insulin sensitivity

\begin{tabular}{|c|c|c|c|c|c|}
\hline Model & Dependent & Input variables & Significant predictors & $\mathrm{R}^{2}$ & $p$-value \\
\hline 1 & GIR & $\begin{array}{c}\mathrm{VO}_{2 \max } \\
{ }^{14} \mathrm{C} \text { palmitate oxidation } \\
\text { PLIN5+ LD size } \\
\text { PLIN5- LD size }\end{array}$ & $\mathrm{VO}_{2 \max }$ & 0.702 & $<0.001$ \\
\hline 2 & GIR & $\begin{array}{c}\mathrm{VO}_{2 \max } \\
{ }^{14} \mathrm{C} \text { palmitate oxidation } \\
\text { PLIN5 protein content }\end{array}$ & $\begin{array}{c}\mathrm{VO}_{2 \max } \\
\text { PLIN5 protein content }\end{array}$ & 0.747 & $<0.001$ \\
\hline 3 & GIR & $\begin{array}{c}\mathrm{VO}_{2 \max } \\
{ }^{14} \mathrm{C} \text { palmitate oxidation } \\
\text { PLIN5+ LD number } \\
\text { PLIN5- LD number }\end{array}$ & $\begin{array}{c}\mathrm{VO}_{2 \max } \\
\text { PLIN5+ LD number }\end{array}$ & 0.807 & $<0.001$ \\
\hline
\end{tabular}

\section{DISCUSSION}

Given previous observations of PLIN5 being a lipid droplet coat protein favoring benign IMCL storage we hypothesized that -with similar IMCL content- the fraction of PLIN5+ LDs is a determinant of skeletal muscle insulin sensitivity and may well be the underlying factor explaining the athlete's paradox. Thus, we investigated if the morphology of PLIN5 coated LDs is a determinant of insulin sensitivity and related parameters (like maximal oxidative capacity and the ability to oxidize fatty acids). Upon targeting similar IMCL content in Trained and T2DM, PLIN5 content and insulin sensitivity was significantly higher in Trained than in T2DM. A straightforward interpretation of this could be that in the face of similar IMCL content, having high PLIN5 content is indeed paralleled by high insulin sensitivity and low PLIN5 with low insulin sensitivity. However, PLIN5 content measured by Western blotting did not correlate with peripheral insulin sensitivity as measured by glucose infusion rate. Upon making the distinction between PLIN5+ and PLIN5- LDs we observed that, 
although lipid area fraction of PLIN5+ LDs was similar in Trained and T2DM, Trained had significantly more PLIN5+ LDs than any of the other groups, whereas LD size was similar across groups. Although we observed no correlation between the number of PLIN5+ LDs and insulin sensitivity, stepwise regression analysis revealed that next to oxidative capacity PLIN5 content and the number of PLIN5+ LDs significantly added to the correlation. We observed that trained subjects have a different pattern of muscle fat storage than T2DM patients which is unaffected by PLIN5 protein content. In addition, PLIN5 coated IMCL was related with maximal oxidative capacity and the ability to oxidize fatty acids. However, PLIN5 coated LDs and PLIN5 protein content were not associated with insulin sensitivity. This has led us to conclude that the trained state and the associated increased PLIN5 content and number of PLIN5+ LDs, appears not to affect the lipid storage pattern, but only is a determinant of insulin sensitivity when oxidative capacity is taking into account.

In the pre-PLIN5 era, several studies have shown that a different pattern of lipid storage is associated with insulin sensitivity $[13,17]$. A weight loss induced improvement in insulin sensitivity is associated with a reduction in LD size, while total IMCL content was maintained [13]. In contrast, at the same time it was shown that LD size was not different between athletes and T2DM patients, but that LD number was higher in the athletes [17]. In this study population, however, athletes possessed higher IMCL content than T2DM. By matching trained and T2DM subjects for IMCL content (but over a wide range of insulin sensitivity and oxidative capacity) we confirmed previous observations that trained have similar LD size but a higher LD number than patients with T2DM.

In experimental models, overexpression of PLIN5 promoted oxidative gene expression and mitochondrial lipid droplet interaction [9]. In humans, PLIN5 protein content has been associated with a high oxidative capacity $[6,16]$ and endurance training up-regulates PLIN5 protein content [1820]. Conversely, 2 weeks of immobilization resulted in a drop in PLIN5 content and markers of oxidative capacity, which was not restored upon 6 weeks of endurance training [21]. By using a microscopic approach, we were able to differentiate between PLIN5+ and PLIN5- LDs. PLIN5 coated LDs where higher in number and larger than LDs devoid of PLIN5 in all groups. Others have shown that 6 weeks of endurance training induces an increase in number of LDs associated with PLIN5 [19]. This is in accordance with our findings showing that the number of PLIN5+ LDs in the trained state is almost double the number of the Lean, Obese or T2DM. Interestingly, we observed that PLIN5 protein content, the lipid area fraction of PLIN5+ LDs as well as the number of PLIN5+ LDs correlates positively with $\mathrm{VO}_{2 \max }$, no such correlations were present for PLIN5- LDs. Thus, a high maximal oxidative capacity, a recognized determinant of insulin sensitivity $[22,23]$, coincides with high levels of PLIN5 coated IMCL. 
Oxidative capacity measured by indirect calorimetry reflects mitochondrial oxidative capacity. PLIN5 protein content is associated with mitochondrial fat oxidative capacity and overexpression results in a more efficient fatty acid oxidation [7]. This is in agreement with our results showing an association between PLIN5 protein content and with the ex vivo capacity to oxidize fatty acids. Several studies have observed PLIN5 in the mitochondrial fraction $[7,8,24]$. Although overexpressing PLIN5 in a cell model induces a physical link between LDs and mitochondria and regulates LD lipolysis and mitochondrial fatty acid oxidation [8], the role of PLIN5 in regulating lipolysis remains controversial. Studies have demonstrated that PLIN5 reduces LD lipolysis [8] or facilitates the interaction between ATGL and CGI-58 and thereby tunes lipolytic rate to fat oxidation [25]. We here report that PLIN5 protein content measured in lysates associates positively with the ability to oxidize palmitate. More specifically, we showed that the PLIN5+ lipid area fraction and the number of PLIN5+ LDs appears responsible for this association.

We previously observed that in endurance trained athletes PLIN5 protein content is a predictor of insulin sensitivity [16], whereas whole body PLIN5 deletion results in insulin resistance [14]. These data may indicate that PLIN5 content either directly or indirectly affects insulin sensitivity. Our study confirms previous observations that PLIN5 protein content in trained subjects is higher than in Lean, Obese or T2DM $[6,16,21]$. In contrast to previous work [16, 19], however, we did not observe a direct association between PLIN5 protein content and insulin sensitivity. Rather we showed by stepwise regression that $70.2 \%$ of the variation in insulin sensitivity could be explained by $\mathrm{VO}_{2 \max }$ but that inclusion of PLIN5 protein content increased the predictive value to $74.7 \%$ with a further increase in predictive value (80.7\%) if the number of PLIN5+ LDs was taken into account. However, PLIN5 is not only localized to the LDs, but also to other fractions like mitochondria $[7,8,24]$. PLIN5 regulates lipolysis and thereby prevents lipotoxicity [26] and thus potentially protects against insulin resistance. In addition, we observed recently that a high number of PLIN5 coated LDs were protective against fasting-induced insulin resistance [11]. Though, in the present study we did not observe an association between insulin sensitivity and PLIN5 coated IMCL or number of PLIN5+ LDs. This indicates that PLIN5 does not directly elucidate the athlete's paradox despite higher PLIN5 protein levels in endurance trained athletes who are very insulin sensitive and have a high oxidative capacity. High levels of PLIN5 suggest a more dynamic LD, which is beneficial for insulin sensitivity. However, for a dynamic LD the interaction of PLIN5 with ATGL and/or CGI-58 might be of more importance than just high PLIN5 protein levels.

In conclusion, we demonstrate that abundance of PLIN5 in skeletal muscle of trained athletes relative to patients with type 2 diabetes cannot explain the athlete's paradox in a direct and 
straightforward manner. Although coating of lipid droplets with PLIN5 clearly associated with factors favorably affecting insulin sensitivity (a high whole body oxidative capacity and mitochondrial fatty acid oxidation), the predictive value of PLIN5 for muscle insulin sensitivity was only present in the face of a high oxidative capacity.

\section{REFERENCES}

1. Pan, D.A., et al., Skeletal muscle triglyceride levels are inversely related to insulin action. Diabetes, 1997. 46(6): p. 983-8.

2. Krssak, M., et al., Intramyocellular lipid concentrations are correlated with insulin sensitivity in humans: a 1H NMR spectroscopy study. Diabetologia, 1999. 42(1): p. 113-6.

3. Goodpaster, B.H., et al., Intramuscular lipid content is increased in obesity and decreased by weight loss. Metabolism, 2000. 49(4): p. 467-72.

4. Goodpaster, B.H., et al., Skeletal muscle lipid content and insulin resistance: evidence for a paradox in endurance-trained athletes. J Clin Endocrinol Metab, 2001. 86(12): p. 5755-61.

5. Phielix, E., et al., High oxidative capacity due to chronic exercise training attenuates lipid-induced insulin resistance. Diabetes, 2012. 61(10): p. 2472-8.

6. Amati, F., et al., Skeletal muscle triglycerides, diacylglycerols, and ceramides in insulin resistance: another paradox in endurance-trained athletes? Diabetes, 2011. 60(10): p. 2588-97.

7. Bosma, M., et al., The lipid droplet coat protein perilipin 5 also localizes to muscle mitochondria. Histochem Cell Biol, 2012. 137(2): p. 205-16.

8. Wang, H., et al., Perilipin 5, a lipid droplet-associated protein, provides physical and metabolic linkage to mitochondria. J Lipid Res, 2011. 52(12): p. 2159-68.

9. Bosma, M., et al., Overexpression of PLIN5 in skeletal muscle promotes oxidative gene expression and intramyocellular lipid content without compromising insulin sensitivity. Biochim Biophys Acta, 2013. 1831(4): p. 844-52.

10. Billecke, N., et al., Perilipin 5 mediated lipid droplet remodelling revealed by coherent Raman imaging. Integr Biol (Camb), 2015.

11. Gemmink, A., et al., Decoration of intramyocellular lipid droplets with PLIN5 modulates fastinginduced insulin resistance and lipotoxicity in humans. Diabetologia, 2016. 59(5): p. 1040-8.

12. Schneider, C.A., W.S. Rasband, and K.W. Eliceiri, NIH Image to ImageJ: 25 years of image analysis. Nat Methods, 2012. 9(7): p. 671-5.

13. He, J., B.H. Goodpaster, and D.E. Kelley, Effects of weight loss and physical activity on muscle lipid content and droplet size. Obes Res, 2004. 12(5): p. 761-9.

14. Mason, R.R., et al., PLIN5 deletion remodels intracellular lipid composition and causes insulin resistance in muscle. Mol Metab, 2014. 3(6): p. 652-63.

15. Wolins, N.E., et al., OXPAT/PAT-1 is a PPAR-induced lipid droplet protein that promotes fatty acid utilization. Diabetes, 2006. 55(12): p. 3418-28.

16. Koves, T.R., et al., PPARgamma coactivator-1alpha contributes to exercise-induced regulation of intramuscular lipid droplet programming in mice and humans. J Lipid Res, 2013. 54(2): p. 522-34.

17. van Loon, L.J., et al., Intramyocellular lipid content in type 2 diabetes patients compared with overweight sedentary men and highly trained endurance athletes. Am J Physiol Endocrinol Metab, 2004. 287(3): p. E558-65.

18. Louche, K., et al., Endurance exercise training up-regulates lipolytic proteins and reduces triglyceride content in skeletal muscle of obese subjects. J Clin Endocrinol Metab, 2013. 98(12): p. 4863-71. 
19. Shepherd, S.O., et al., Sprint interval and traditional endurance training increase net intramuscular triglyceride breakdown and expression of perilipin 2 and 5. J Physiol, 2013. 591(3): p. 19.

20. Peters, S.J., et al., Perilipin family (PLIN) proteins in human skeletal muscle: the effect of sex, obesity, and endurance training. Appl Physiol Nutr Metab, 2012. 37(4): p. 724-35.

21. Vigelso, A., et al., Effects of immobilization and aerobic training on proteins related to intramuscular substrate storage and metabolism in young and older men. Eur J Appl Physiol, 2016. 116(3): p. 481-94.

22. Bruce, C.R., et al., Muscle oxidative capacity is a better predictor of insulin sensitivity than lipid status. J Clin Endocrinol Metab, 2003. 88(11): p. 5444-51.

23. Tonino, R.P., Effect of physical training on the insulin resistance of aging. Am J Physiol, 1989. 256(3 Pt 1): p. E352-6.

24. Ramos, S.V., et al., Changes in mitochondrial PLIN3 and PLIN5 protein content in rat skeletal muscle following endurance training and acute stimulated contraction. Exp Physiol, 2015.

25. Granneman, J.G., et al., Functional interactions between Mldp (LSDP5) and Abhd5 in the control of intracellular lipid accumulation. J Biol Chem, 2009. 284(5): p. 3049-57.

26. Kuramoto, K., et al., Perilipin 5, a lipid droplet-binding protein, protects heart from oxidative burden by sequestering fatty acid from excessive oxidation. J Biol Chem, 2012. 287(28): p. 23852-63. 


\title{
CHAPTER 6
}

\section{Different intramyocellular lipid storage pattern and level of lipid droplet saturation in athletes and type 2 diabetes patients}

\author{
Anne Gemmink ${ }^{*}$, Sabine Daemen ${ }^{*}$, Nils Billecke, Katrina Rieger, Bram \\ Brouwers, Gert Schaart, Frederik Fleissner, Joris Hoeks, Patrick Schrauwer, \\ Sapun H. Parekh, Matthijs K.C. Hesselink \\ In preparation \\ *These authors contributed equally to this work
}




\section{CHAPTER 6}

\section{ABSTRACT}

Endurance trained athletes (Trained) and type 2 diabetes (T2DM) patients have similar intramyocellular lipid (IMCL) content, but differ in levels of insulin sensitivity; the so-called athlete's paradox. Lipid droplet (LD) size, number, location and lipid composition are suggested parameters to explain the athlete's paradox. Lipid composition is mostly measured in lipid extractions from muscle tissue. Here we applied Coherent Anti-stokes Raman Spectroscopy (CARS) to measure the chemical composition of LDs in a fiber type specific manner. In addition, we determined LD size, number and location per fiber type. We hypothesized that these parameters are involved in the athlete's paradox.

Muscle biopsies were taken from 8 Trained, lean sedentary, obese participants and T2DM patients. Trained and T2DM were matched for total IMCL content and muscle biopsies were analyzed for LD size, number and location with confocal microscopy. Chemical LD composition was determined with CARS microscopy in combination with immunofluorescence to determine fiber type for Trained and T2DM.

Differences in LD morphology and location where mainly observed in type II fibers. T2DM had larger LDs $\left(0.24 \pm 0.02 \mu \mathrm{m}^{2}\right.$ vs. $\left.0.37 \pm 0.05 \mu \mathrm{m}^{2}\right)$ and more SS IMCL content due to more $(10.0 \pm 1.5 \%$ vs. $21.7 \pm 1.8 \%)$ and larger LDs $\left(0.24 \pm 0.02 \mu \mathrm{m}^{2}\right.$ vs. $\left.0.47 \pm 0.05 \mu \mathrm{m}^{2}\right)$ in type II fibers than Trained. Trained stored their excess IMCL mainly in type I fibers as a result of more LDs $\left(0.057 \pm 0.008 \mu^{-2}\right.$ vs. $0.031 \pm 0.004 \mu \mathrm{m}^{-2}$ ). LDs of Trained contained slightly more unsaturated carbons in type II fibers $(0.79 \pm 0.03$ vs. $0.67 \pm 0.04)$ than T2DM, but chain length was similar (15.99 \pm 0.04 vs. $16.35 \pm 0.26)$. LD size in type II fibers correlated negatively with insulin sensitivity $(r=-0.398, p<0.05)$.

In conclusion, LD size and IMCL content in the SS region of type II fibers are important players in the athlete's paradox. If the chemical LD composition is of relevance for the athlete's paradox should be further examined. 


\section{INTRODUCTION}

Surplus intramyocellular lipid (IMCL) content is considered to hamper insulin sensitivity [1-3]. However, high levels of IMCL are also observed in endurance trained athletes who are rather insulin sensitive, a phenomenon known as the athlete's paradox [4]. This has led to the view that the excess IMCL content (mainly consisting of triacylglycerol) in obesity and type 2 diabetes (T2DM) is rather a surrogate marker for the amount of lipid intermediates, like diacylglycerol and ceramides. Diacylglycerol can interfere with insulin signaling via PKC activation $[5,6]$ as well as ceramides [7]. Lipid infusion in healthy lean participants results in insulin resistance, an increase in diacylglycerol and PKC [8]. However, the relationship between the presence of lipid or lipid intermediates and insulin sensitivity is not straightforward. Amati et al. 2011 [9] has reported higher levels of saturated DAG in athletes relative to age matched lean sedentary and obese participants. In contrast, Bergman et al. 2010 [10] showed higher levels of saturated DAG in lean sedentary than in athletes.

Conventionally, studies examining lipid intermediates perform mass spectrometry analysis on lipids extracted from e.g. muscle biopsy samples. However, this does not provide information about the location of the lipid intermediates. To obtain insight into the composition of individual lipid droplets (LDs) Coherent Anti-stokes Raman Spectroscopy (CARS) can be applied. CARS microscopy gives spatial information on chemical groups in a pre-defined location. Application of CARS microscopy to muscle samples unilaterally overexpressing the lipid droplet coat protein perilipin 5 (PLIN5) revealed that in muscle overexpressing PLIN5 the lipid droplet composition was different from the contralateral control muscle [11]. As trained athletes have higher levels of PLIN5 than untrained patients with type 2diabetes $[9,12]$. Combining these observations has led us to suggest that the composition of lipid droplets in muscle samples from trained athletes is different from the composition of lipid droplets in muscles of patients with type 2 diabetes.

Other parameters postulated to explain the athlete's paradox are LD morphology (like number and size) as well as the subcellular location of the droplets. Over a decade ago, He et al. 2004 [13] showed that upon a 16-week combined exercise and weight loss intervention, the observed reduction in LD size associated with improvements in insulin sensitivity in obese sedentary participants. Moreover, it has been shown that T2DM patients had larger LDs compared to lean and obese sedentary participants in type I fibers, whereas no such differences were observed for type II fibers. At about the same time, however, van Loon et al. 2004 [14] showed no difference in LD size between endurance trained athletes, T2DM patients and normoglycemic overweight sedentary participants, but reported a higher LD number in type I fibers in the athletes. 
These LDs can be located in the subsarcolemmal (SS) or in the intermyofibrillar (IMF) regions. Image analyses based upon electron microscopy revealed that the fraction of LDs in the subsarcolemmal space associated with insulin resistance [15]. In addition, it has been reported that endurance training results in a reduced SS IMCL content [15], as well as a reduction in LD size and number [16, 17].

Our in-home developed protocols now permit simultaneous examination of the chemical composition of LDs, LD size and number, and subcellular LD distribution, all in a fiber type specific manner. This permits us to explore these parameters in muscle biopsies from in endurance trained athletes and T2DM patients to get more insight into the factors contributing to the athletes' paradox. From our database we semi-randomly selected participants with a wide range of insulin sensitivity but similar IMCL content. We hypothesized that despite similar levels of IMCL, highly insulin sensitive participants (trained athletes) store their IMCL differently with respect to morphology, subcellular distribution, location and chemical composition.

\section{METHODS}

\section{Participants}

From our database of endurance-trained athletes (Trained; $\mathrm{VO}_{2 \max }>55 \mathrm{ml} / \mathrm{kg} / \mathrm{min}$ ), lean sedentary (Lean; $\mathrm{VO}_{2 \max }<45 \mathrm{ml} / \mathrm{kg} / \mathrm{min}$ ), obese sedentary (Obese) and T2DM participants, eight participants per group were semi-randomly selected for inclusion. Prior to inclusion in this study, we screened myocellular lipid content and selected participants based upon similar IMCL content. All participants gave written informal consent before participating in this study. After an overnight fast a muscle biopsy was taken from the $\mathrm{m}$. vastus lateralis. The muscle biopsy was directly frozen in melting isopentane and stored at $-80^{\circ} \mathrm{C}$ until histochemical analyses. After the muscle biopsy a two-step hyperinsulinemic-euglycemic clamp was performed (10 and $40 \mathrm{mU} / \mathrm{m}^{2} / \mathrm{min}$ of insulin). Glucose infusion rate (GIR) during the high insulin infusion was used as a measures for skeletal muscle insulin sensitivity. Body composition was measured with a DXA scan and plasma glucose was determined. In addition, to determine oxidative capacity a graded maximal cycling test was executed until exhaustion. Oxygen consumption was measured throughout the test via indirect calorimetry (Omnical, Maastricht, The Netherlands). Maximal oxygen consumption was expressed per kg lean body mass to correct for differences in fat mass. 


\section{Coherent Anti-stokes Raman Spectroscopy}

Lipid droplet composition was measured in a fiber type specific fashion in Trained and T2DM, respectively representing the higher and lower extremes of hyperinsulinemic euglycemic clamp based insulin sensitivity. Four $\mu \mathrm{m}$ thick sections were mounted on coverslips. Before the application of CARS, sections were stained against laminin (L9393, Sigma, St. Louis, USA) and myosin heavy chain type I (A4.840, Developmental Studies Hybridoma Bank, lowa City, IA, USA) as described below and sections were mounted in PBS and sealed immediately with nail polish to avoid dehydration. Scout images of the sections were acquired on an Olympus IX80 inverted microscope using Cell F imaging software to identify type I and type II fibers and to select these fibers to be imaged with CARS. Samples were imaged as described before [11]. In short, a dual-output laser source (Leukos-CARS, Leukos) provided the pump and stokes beams. The pump beam had a wavelength of $1064 \mathrm{~nm}$ and the stokes beam from $1050 \mathrm{~nm}$ to $1600 \mathrm{~nm}$. Both lasers were routed on an optical table to excite the sample mounted on a stepper-motor stage (Microstage, Mad City Labs) with a nested piezo stage (Nano-PDQ 375 HS, Mad City Labs) at the same time. A selected area with a type I and type II fiber was scanned in 9 tiles of $25 \mu \mathrm{m}$ by $25 \mu \mathrm{m}$ resulting in a field of view of $75 \mu \mathrm{m}$ by $75 \mu \mathrm{m}$. Per participant three field of views were imaged. CARS spectra were acquired between 600 and 3400 $\mathrm{cm}^{-1}$. The filtered CARS signal detected by a deep-depletion CCD (Newton DU920P-BR-DD, Andor). Custom written software in Labview (National Instruments) was used to control the CARS microscope.

\section{Extraction of characteristic LD spectra}

Raw CARS spectra for all 16 patients (8 T2DM and 8 Trained) were analyzed using IgorPro 6.37 (Wavemetrics) and converted into Raman-like spectra as previously described [18] with phasedetrending to calculate the final $\operatorname{Im}\left\{X^{(3)}\right\}$, which is directly proportional to the intensity from a spontaneous Raman spectrum [19]. Following this process, we initially constructed images based upon the intensity of $\mathrm{CH}_{2}$ by integrating the Raman-like signal from $2840-2855 \mathrm{~cm}^{-1}$ and plotted this integrated value for each spatial pixel. The resultant $\mathrm{CH}_{2}$ images were then further analyzed by creating regions of interest (ROIs) to separate by fiber type (as identified by fluorescence labeling). For each ROI, a sweeping threshold was used to obtain an array of images reflecting differing levels of lipid signals. These images started with a low threshold, where the image was composed of LDs and the surrounding tissue, and ended with a high intensity where the image contained only the brightest LDs. For each thresholded image, we calculated an average spectrum of those pixels with 
values above the threshold. These resultant spectra (at least 40 spectra (thresholds) per data set) were first masked, where intensities from the so-called "quiescent region" (1800-2750 $\left.\mathrm{cm}^{-1}\right)$ were removed. The remaining regions: the "fingerprint" (900-1800 cm $\left.{ }^{-1}\right)$ and " $\mathrm{CH}$ stretch" $\left(2750-3150 \mathrm{~cm}^{-}\right.$ $\left.{ }^{1}\right)$ were used as inputs into a Multivariate Curve Resolution (MCR) analysis program to derive chemical "components" in our data.

The MCR-derived LD and non-LDs tissue spectra were calculated for each data set and normalized to the amplitude of the derived spectrum at $1440 \mathrm{~cm}^{-1}\left(\mathrm{CH}_{2}\right.$ deformation) and then averaged by fiber type for each patient. These averaged LD and non-LD spectra were representative spectra for each fiber type for each patient. The patients were then grouped into their treatment groups (T2DM and athlete) for comparison between fiber types and between groups.

\section{Calculating average chain in LDs}

To determine the chain length, the ratio of the integration of the peak at $1650 \mathrm{~cm}^{-1}(1659.72-1633.4$

$\left.\mathrm{cm}^{-1}\right)$ over the peak at $1440\left(1449.69-1427.57 \mathrm{~cm}^{-1}\right)$ was determined. This value was compared to those derived from standard curves from fatty acids and the chain length was calculated using the method of Wu et al. [20].

\section{Histochemical analyses}

To determine IMCL content, LD size and number of LDs $7 \mu \mathrm{m}$ thick sections were cut and mounted on glass slides. On each slide a section from each group was mounted to reduce variability in staining intensities between groups. Bodipy 493/503 (D3922, Molecular Probes, Leiden, The Netherlands) was used to visualize intramyocellular LDs. Cryosections were fixed with $3.7 \%$ formaldehyde for 30 min. After blocking with blocking buffer (150 mM NaCl, $20 \mathrm{mM}$ Tris $\mathrm{pH} 6.8$ and 2\% BSA) and permeabilization with 0.25\% TX-100 (648466, Merck, Darmstadt, Germany), sections were incubated with primary antibodies against Laminin and myosin heavy chain type I for $60 \mathrm{~min}$. Subsequently, sections were incubated for $1.5 \mathrm{~h}$ with the appropriate secondary antibodies conjugated with AlexaFluor 405 and AlexaFluor 555 and Bodipy 493/503 1:100 at $37^{\circ} \mathrm{C}$. Sections were mounted in Mowiol with \#1 coverslips and stored in the dark until imaging. 


\section{Image acquisition and analysis}

For the analysis of IMCL content and a more detailed analysis of IMCL content regarding LD size and number of LDs cross-sectional images were taken on a Leica TCS SPE confocal microscope using a 63x 1.3 N.A. oil immersion objective and a 1.1 optical zoom at 2048 by 2048 pixels. Laminin, myosin heavy chain type I and Bodipy 493/503 were imaged using the $405 \mathrm{~nm}, 532 \mathrm{~nm}$ and $488 \mathrm{~nm}$ laser lines, respectively. Type I and type II fibers were identified based on myosin heavy chain type I staining. The fiber type ratio was determined using a Nikon E800 fluorescence microscope before confocal imaging to prevent bias by differences in fiber type ratio. For each participant, crosssections of 20 fibers were imaged with the confocal microscope with the determined type I to type II ratio. After image acquisition images were deconvolved using Huygens Essential software (Scientific Volume Imaging B.V., Hilversum, The Netherlands) to improve signal-to-noise ratio and remove background. For each fiber type lipid area fraction, LD size and number of LDs relative to muscle fiber area were analyzed by using ImageJ [21]. LD size distribution and LD-membrane distances were calculated with Matlab R2012a (The Mathworks, Inc., Natick, Massachusetts, USA). Based on these LD-membrane distances LDs were separated in LDs located in near vicinity of the cell membrane (SS LDs) or LDs located more towards the core of the myofiber (IMF LDs). This was done based on LDmembrane distance which was corrected for the longest LD-membrane distance in each muscle fiber to correct for differences in cell size. This resulted in widths for the SS region between $0.61 \mu \mathrm{m}$ and $3.08 \mu \mathrm{m}$.

\section{Statistics}

Results are presented as mean \pm SEM. Statistical analysis were performed using SPSS version 21.0 (SPSS, Chicago, IL, USA). Statistical differences between groups and fiber types were tested with a Repeated Measures ANOVA with fiber type as a within subject factor for lipid droplet morphology. If the interaction effect for fiber type was significant a One-way ANOVA was used to test for statistical differences between groups for each fiber type. A Bonferroni post-hoc test was performed to specify which groups statistically differed. A paired samples t-test was used to test for each group significant differences between fiber types. Pearson's correlation coefficients were used to test for significant linear association between variables. An unpaired two sample t-test was performed between sample groups (T2DM and Athlete or Type I and Type II) for comparing spectra between groups. $p<0.05$ was considered to be significant. 


\section{RESULTS}

\section{Participant characteristics}

Participant characteristics are shown in Table 1. Obese and T2DM participants were significantly older and had a higher BMI and percentage fat mass than Trained and Lean. T2DM patients had higher fasting glucose levels compared to the other groups (Trained: $5.14 \pm 0.09$; Lean: $5.24 \pm 0.10$; Obese: $4.91 \pm 0.28$; T2DM: $7.43 \pm 0.53 \mathrm{mmol} / \mathrm{L}, p<0.01)$. Due to differences in percentage fat mass, $\mathrm{GIR}$

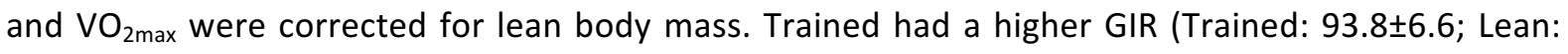
70.4 \pm 5.7 ; Obese: $38.0 \pm 2.9$; T2DM: $25.7 \pm 5.3 \mu \mathrm{mol} / \mathrm{kg}_{\mathrm{LBM}} / \mathrm{min}, p<0.01$ ) and $\mathrm{VO}_{2 \max }$ (Trained: 71.0 \pm 1.6 ; Lean: $51.8 \pm 1.8$; Obese: $39.8 \pm 1.9$; T2DM: $36.8 \pm 1.5 \mathrm{~mL} \mathrm{O} / \mathrm{kg}_{\mathrm{LBM}} / \mathrm{min}, p<0.01$ ) compared to other groups. Obese and T2DM participants had a lower GIR and oxidative capacity compared to Lean $(p<0.01)$.

Table 1. Participant characteristics

\begin{tabular}{lcccc}
\hline Parameter & Athletes & Lean & Obese & T2DM \\
\hline Age (years) & $26.0 \pm 1.8$ & $23.5 \pm 1.2$ & $54.1 \pm 3.1^{*+}$ & $60.6 \pm 2.0^{*+}$ \\
Body weight $(\mathrm{kg})$ & $72.4 \pm 2.6$ & $73.1 \pm 2.3$ & $96.1 \pm 3.3^{*+}$ & $95.9 \pm 2.8^{*+}$ \\
$\%$ Fat mass & $13.4 \pm 0.5$ & $18.4 \pm 1.5^{*}$ & $28.9 \pm 0.9^{*+}$ & $28.3 \pm 0.9^{*+}$ \\
BMI $\left(\mathrm{kg} / \mathrm{m}^{2}\right)$ & $21.0 \pm 0.6$ & $22.2 \pm 0.6$ & $29.4 \pm 0.4^{*+}$ & $29.6 \pm 0.8^{*+}$ \\
Fasting glucose $(\mathrm{mmol} / \mathrm{L})$ & $5.14 \pm 0.09$ & $5.24 \pm 0.10$ & $4.91 \pm 0.28$ & $7.43 \pm 0.53^{*+\neq}$ \\
GIR $(\mu \mathrm{mol} / \mathrm{kg}$ LBM $/ \mathrm{min})$ & $93.8 \pm 6.6$ & $70.4 \pm 5.7^{*}$ & $38.0 \pm 2.9^{*+}$ & $25.7 \pm 5.3^{*+}$ \\
Maximal aerobic capacity $\left(\mathrm{ml} \mathrm{O} / \mathrm{kg}_{\text {LBM }} / \mathrm{min}\right)$ & $71.0 \pm 1.6$ & $51.8 \pm 1.8^{*}$ & $39.8 \pm 1.9^{*+}$ & $36.8 \pm 1.5^{*+}$ \\
\hline
\end{tabular}

${ }^{*} p<0.05$ vs. Trained; $+p<0.05$ vs. Lean; $\ddagger p<0.05$ vs. Obese

\section{Lipid area fraction}

Lipid area fraction determined by confocal microscopy showed no differences between Trained and T2DM patients $(1.06 \pm 0.12 \%$ vs. $0.73 \pm 0.13 \%, p=0.274$; Figure 1$)$. Lean and Obese had lower lipid area fraction than Trained $(0.53 \pm 0.14 \%$ and $0.43 \pm 0.06 \%$ lean and obese respectively, $p<0.05)$. Interestingly, lipid droplet storage displayed fiber type specificity with trained having higher lipid area fraction in type I fibers than T2DM patients $(1.49 \pm 0.19 \%$ vs. $0.86 \pm 0.14 \%, p<0.05)$, while for type II fibers there was a tendency for a group effect (Trained: $0.42 \pm 0.08 \%$; Lean: $0.29 \pm 0.08 \%$; Obese: $0.31 \pm 0.08 \%$; T2DM: $0.65 \pm 0.14 \%, p=0.062$ ). Moreover, in trained the lipid area fraction was higher in type I fibers than in type II fibers $(p<0.05)$, whereas for T2DM patients lipid area fraction was comparable for both fiber types $(p=0.12)$. 


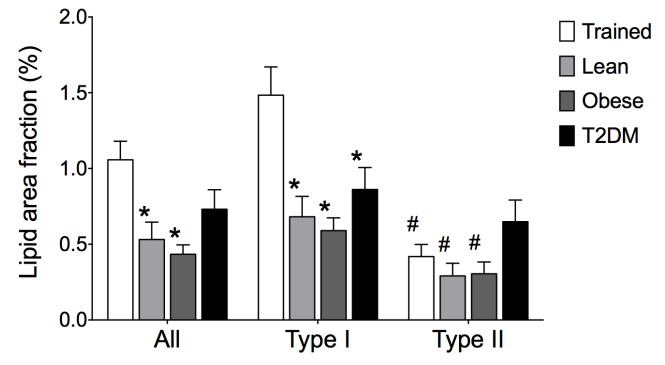

Figure 1. Lipid area fraction of all fiber types, type I fibers, and type II fibers of Trained, Lean, Obese and T2DM; * $p<0.05$ vs. Trained; \# $p<0.05$ vs. Type I fibers.

\section{Lipid droplet composition}

CARS microscopy was performed in Trained and T2DM, the two most extremes for insulin sensitivity. There were subtle differences in the chemical LD composition regarding the level of unsaturation. Trained had slightly more double bound carbons in their acyl chains within the LDs than T2DM $(0.80 \pm 0.03$ vs. $0.69 \pm 0.04 ; p<0.05$; Figure $2 \mathrm{~A})$. This was particularly true for the type II fibers $(0.79 \pm 0.03$ vs. $0.67 \pm 0.04 ; p<0.05$; Figure $2 \mathrm{~A})$. Chain length did not differ between Trained and T2DM (Figure 2B).

A
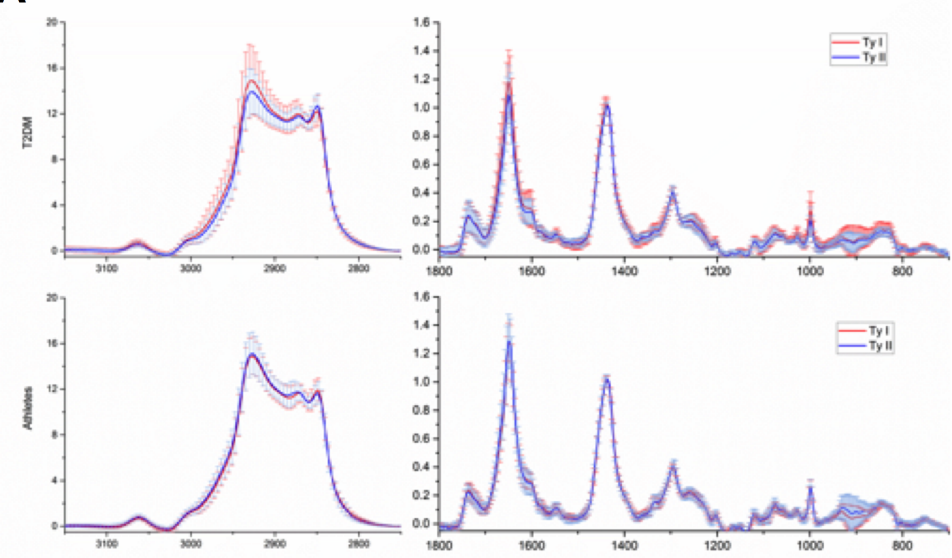

Wavenumber $\left(\mathrm{cm}^{-1}\right)$

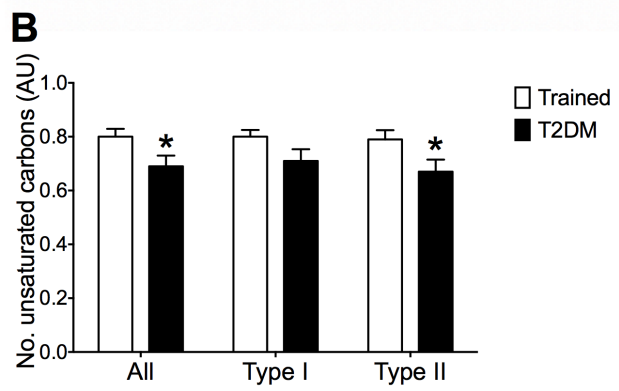

\section{C}

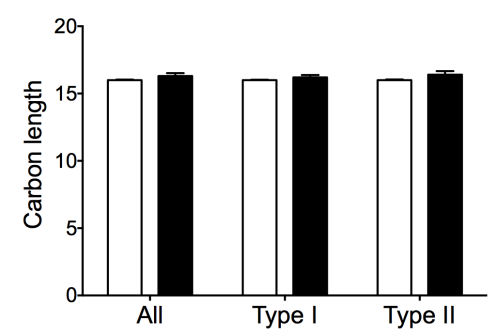

Figure 2. Chemical composition of intramyocellular lipid droplets. (A) Average spectra from pixels belonging to lipid droplets obtained by CARS. Spectra of T2DM patients and Trained are very similar with subtle differences. (B) Average number of unsaturated carbons; * $p<0.05$ vs. Trained. (C) Average chain length of fatty acids.

\section{Lipid droplet morphology}

Factors like lipid droplet size and number putatively play a role in the athlete's paradox since improvements in insulin sensitivity upon a training intervention were associated with a reduction in 
LD size [13]. In type II fibers, T2DM patients had significantly larger LDs than Trained $\left(0.24 \pm 0.02 \mu \mathrm{m}^{2}\right.$ vs. $0.37 \pm 0.05 \mu \mathrm{m}^{2}, p<0.05$; Figure $3 \mathrm{~A}$ and $3 \mathrm{~B}$ ), whereas no differences were observed for size among the groups in type I fibers $(p=0.295)$. In patients with T2DM the LDs in type II fibers also were larger than those in type I fibers $\left(0.28 \pm 0.02 \mu \mathrm{m}^{2}\right.$ vs. $\left.0.37 \pm 0.05 \mu \mathrm{m}^{2}, p<0.05\right)$, while Trained had significantly smaller LDs in type II fibers $\left(0.27 \pm 0.01\right.$ vs. $0.24 \pm 0.02 \mu \mathrm{m}^{2}, p<0.05$; Figure $\left.3 \mathrm{~B}\right)$. Trained also had significantly more LDs in type I fibers compared to the other groups (Trained: $0.057 \pm 0.008 \mu^{-2}$; Lean: $0.028 \pm 0.004 \mu \mathrm{m}^{-2}$; Obese: $0.024 \pm 0.003 \mu \mathrm{m}^{-2}$; T2DM: $0.031 \pm 0.004 \mu \mathrm{m}^{-2}, p<0.01$; Figure $4 \mathrm{~A}$ and 4B). All groups had significantly more LDs per $\mu \mathrm{m}^{2}$ in type I fibers compared to type II fibers (Trained: $0.017 \pm 0.003 \mu \mathrm{m}^{-2}$; Lean: $0.011 \pm 0.003 \mu \mathrm{m}^{-2}$; Obese: $0.010 \pm 0.002 \mu \mathrm{m}^{-2}$; T2DM: $0.017 \pm 0.003 \mu \mathrm{m}^{-2}$ for type II fibers, $p<0.01$ ). So, in the face of similar IMCL content in trained and patients with T2DM, trained predominantly store their lipid in numerous normally sized LDs in type I fibers, while in patients with T2DM the lipid is stored in fewer but larger LDs, predominantly in type II fibers.

A

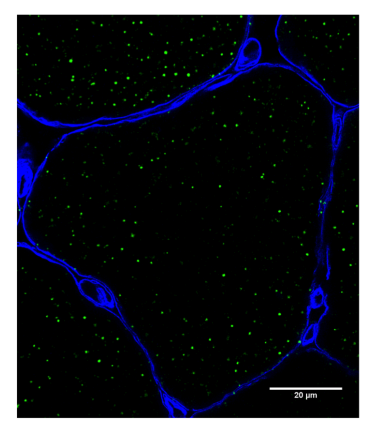

B

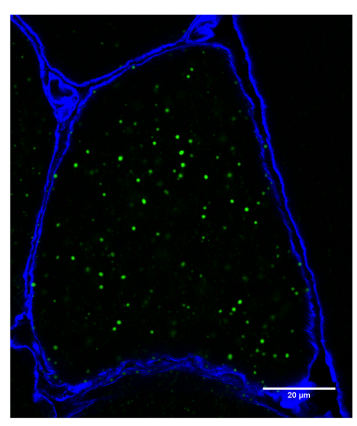

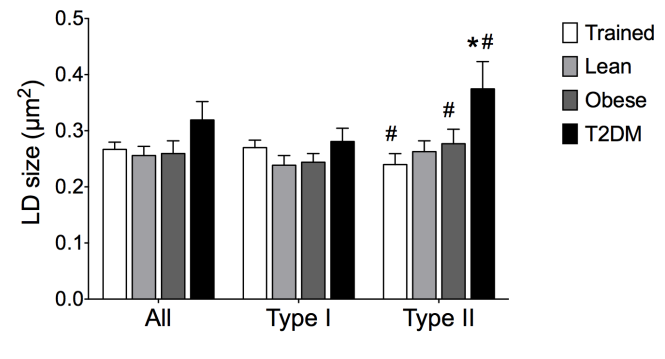

Figure 3. Lipid droplet size of all fibers, type I fibers and type II fibers. (A) Representative images of type II fibers stained for bodipy and laminin of Trained and T2DM. (B) Lipid droplet size quantification; $* p<0.05$ vs. Trained; \# $p<0.05$ vs. Type I fibers.

A

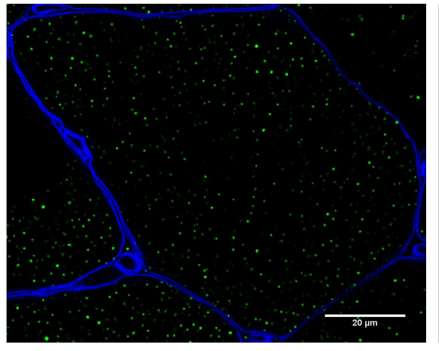

B
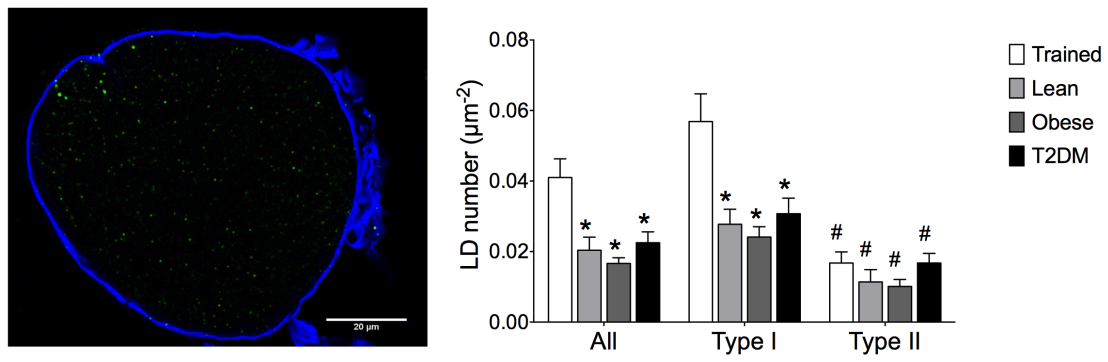

Figure 4. Lipid droplet number of all fibers, type I fibers and type II fibers. (A) Representative images of type I fibers stained for bodipy and laminin of Trained and T2DM. (B) Lipid droplet number quantification; ${ }^{*} p<0.05$ vs. Trained; $\# p<0.05$ vs. Type I fibers. 


\section{Lipid droplet location}

Next we examined if the differences in LD morphology were specific for the SS or IMF region. In all fibers, T2DM participants had a larger fraction of the LDs located in the SS region compared to Trained, whereas Trained had a higher fraction of LDs located in the IMF region. This was more pronounced in the type II fibers (Trained: $10.0 \pm 1.5 \%$; Lean: $16.5 \pm 1.9 \%$; Obese: $20.2 \pm 2.0 \%$; T2DM: $21.7 \pm 1.8 \%$, for SS fraction $p<0.01$; Trained $90.0 \pm 1.5 \%$; Lean: $83.5 \pm 1.9 \%$; Obese: $79.8 \pm 2.0 \%$; T2DM: $78.3 \pm 1.8 \%)$. In type I fibers this pattern in obese and T2DM patients of having a higher percentage of LDs in the SS region was also observed, but failed to reach significance (Figure 5A). Upon examination of both muscle fiber types we observed that size of the LDs in the SS region was significantly larger in patients with T2DM than in trained (Trained: $0.25 \pm 0.02 \mu \mathrm{m}^{2}$; T2DM: $0.41 \pm 0.04$ $\mu \mathrm{m}^{2}$ ). The observation that in the SS region LDs are larger in T2DM patients than in the trained, was most prominent in type II fibers (Trained: $0.24 \pm 0.02 \mu \mathrm{m}^{2}$; T2DM: $0.47 \pm 0.05 \mu \mathrm{m}^{2}$; Figure 5B). Interestingly, in the trained and in type I fibers, LDs in the SS region were smaller than in the IMF region (Trained: $0.26 \pm 0.02 \mu \mathrm{m}^{2}$ in SS vs. $0.29 \pm 0.01 \mu \mathrm{m}^{2}$ in IMF; $p<0.05$; Figure $5 \mathrm{~B}$ ). In patients with T2DM, however, the reversed was observed; LDs in the SS region were larger than in the IMF region (T2DM: $0.37 \pm 0.04 \mu \mathrm{m}^{2}$ in SS vs. $0.31 \pm 0.03 \mu \mathrm{m}^{2}$ in IMF; $p<0.05$; Figure $5 \mathrm{~B}$ ). In type II fibers similar trends were observed. Thus, in T2DM patients the fraction of SS LDs is more than two-fold higher than in the trained state, moreover, these SS LDs are also almost two-fold larger in patients with T2DM. This difference was observed in both fiber types, albeit most pronounced in type II fibers.

A

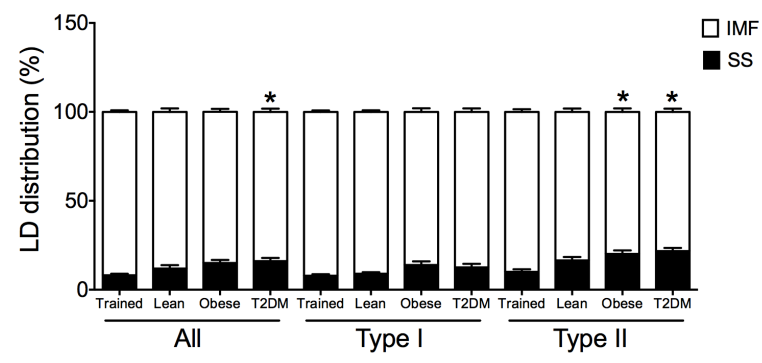

B

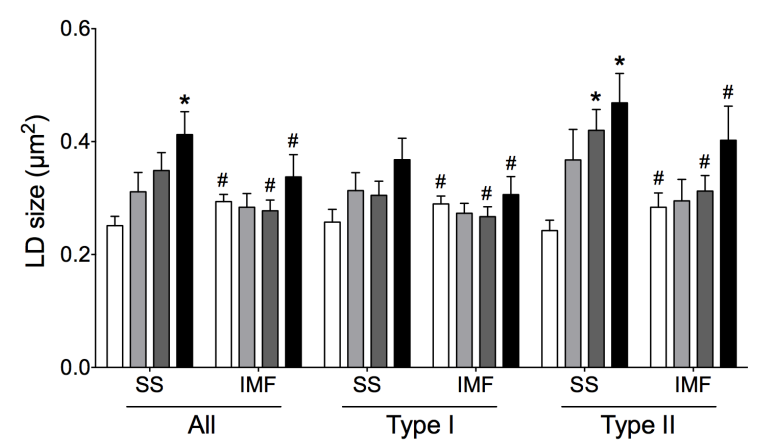

Figure 5. Lipid droplet location for all fibers, type I fibers and type II fibers. (A) Percentage of lipid droplets located near the sarcolemma (SS) and in the intermyofibrillar region (IMF). (B) Size of SS and IMF LDs; ${ }^{*} p<0.05$ vs. Trained; \# $p<0.05$ vs. SS LDs. 


\section{Correlations lipid storage pattern and insulin sensitivity}

Our data morphology data indicates that whilst having similar IMCL content, Trained and T2DM store their fat differently, with Trained storing most of their lipid in type I fibers, in numerous LDs of average size located in the IMF region, whereas T2DM patients store most of their lipid in type II fibers, in fewer but large LDs predominantly located in the SS region. Next we explored if this differential IMCL storage pattern was associated with the differences in insulin sensitivity and oxidative capacity. The GIR correlated negatively with LD size in type II fibers $(r=-0.398, p<0.05)$, but not in type I fibers $(r=-0.012, p=0.949)$. In addition, a higher GIR was associated with more LDs per $\mu \mathrm{m}^{2}$ in type I fibers $(r=0.401, p<0.05)$, but not in type II fibers $(r=-0.073, p=0.690)$ (Figure 6).

A

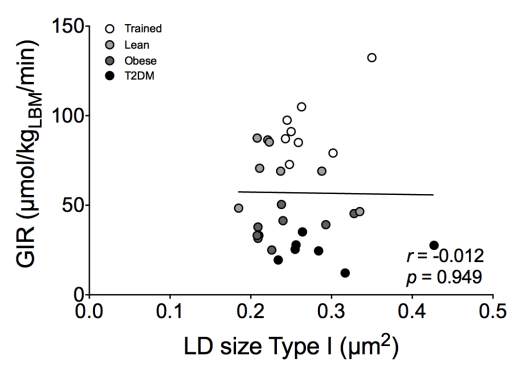

C

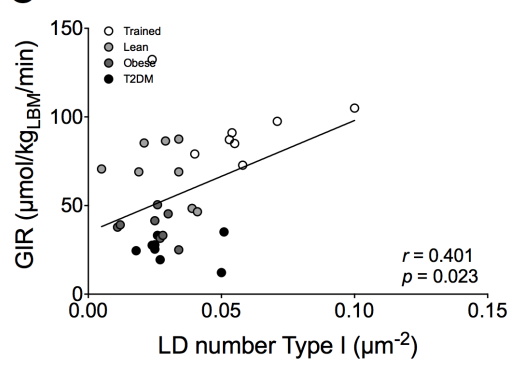

B

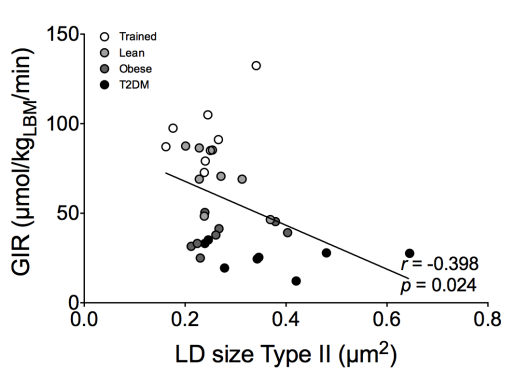

D

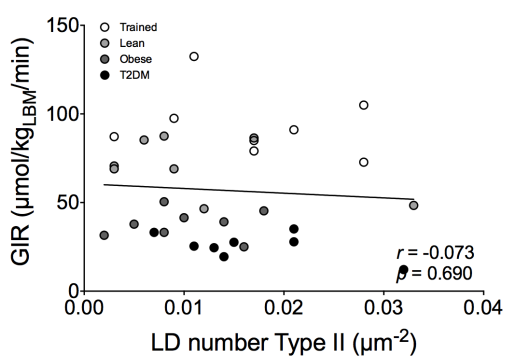

Figure 6. Correlations of LD size and LD number with GIR. Correlations of GIR with LD size of (A) type I fibers and (B) type II fibers. Correlations of GIR with LD number of (C) type I fibers and (D) type II fibers.

\section{Lipid droplet composition of large and small LDs}

The most remarkable difference between Trained and T2DM in lipid storage pattern was the difference in LD size in the type II fibers. As LD size correlates negatively with insulin sensitivity in type II fibers, we explored if the chemical composition of small sized and large LDs in the type II fibers of T2DM were different. This data was obtained from raw CARS spectra upon manually thresholding the LDs. In samples of 2 randomly chosen patients with type 2 diabetes we arbitrarily defined the $20 \%$ largest LDs as large LDs and $20 \%$ of the smallest as small LDs. Upon making this distinction, these preliminary data indicate that large LDs contain acyl chains of similar length but a lower number of double bound carbon atoms, reflecting more saturated fat than small LDs (Table 2). 
Table 2. Chemical composition small vs. large lipid droplets in type II fibers of T2DM patients

\begin{tabular}{lcc}
\hline Parameter & \# unsaturated carbons & Chain length \\
\hline Large & 0.79 & 16.0 \\
Small & 0.90 & 15.9 \\
\hline
\end{tabular}

\section{DISCUSSION}

Here we aimed to examine the contribution of several LD characteristics to the athlete's paradox. Lipid droplet composition, size, number and location all were determined in a fiber type specific manner. Differences were specific for type II fibers. With similar total IMCL content, T2DM patients stored their IMCL in fewer but larger LDs predominantly in the type II fibers in the subsarcolemmal area, whereas athletes store IMCL in more and normal sized LDs in type I fibers, predominantly in the IMF region. The composition of the lipid droplets with respect to chemical bonds that are detectable with CARS was remarkably similar for trained athletes and patients with T2DM. With similar chain length we observed a significantly, but rather modest, lower level of unsaturation predominantly in the type II fibers in patients with T2DM. Interestingly, the LD size in type II fibers and LD number in type I fibers correlated negatively with insulin sensitivity. Having observed this correlation, preliminary post-hoc analysis of the $20 \%$ largest and $20 \%$ smallest LDs in type II fibers revealed that the number of unsaturated carbon bounds was lower in large than in small LDs (suggesting a higher level of saturated acyl chains in large LDs).

Impeded insulin signaling in insulin resistant muscle has long been attributed to (elevated) presence of bioactive lipid derivatives, commonly thought to originate from excess lipid storage in muscle. Bioactive lipids of a wide variety of species are commonly measured by lipidomics. Lipidomic approaches require extraction of lipids of the sample of interest followed by mass spectrometry to quantify the individual lipid species. Using lipidomic approaches so far did not result in conclusive reports on differences in lipid composition in skeletal muscle $[9,10]$. Upon extracting lipids from their native environment, information on composition of individual LDs gets lost.

Novel imaging techniques like CARS, TOF-SIMS and MALDI can provide location specific information about lipid composition. TOF-SIMS and MALDI can result in a very detailed analysis of types of fatty acids stored in LDs. However, at the moment the resolution is not high enough to detect individual LDs [22]. On the other hand, CARS has sufficient resolution to retrieve spectra from individual LDs and gives insights in the chemical composition of the LDs e.g., with respect to the level of saturation and acyl chain length. Upon application of CARS we recently showed that LDs from $\mathrm{m}$. tibialis anterior overexpressing PLIN5 contained more saturated lipids than the contralateral $\mathrm{m}$. tibialis 
anterior expressing endogenous PLIN5 [11]. This underscores the power of CARS to detect differences in the chemical composition of acyl chains in LDs. Here, we observed that in type II fibers, the LDs in Trained contained slightly more unsaturated carbons than T2DM in type II fibers, indicating that the LDs in patients with T2DM contained more saturated acyl chains. This is in line with Manco et al. 2000 [23] who compared normoglycemic lean participants with obese normoglycemic participants and showed a higher unsaturated:saturated ratio of triglycerides extracted from skeletal muscle. Furthermore, Nikolaidis et al. 2004 [24] reported that triglycerides from endurance trained rats contained a slightly lower percentage of mono-unsaturated and a slightly higher percentage of poly-unsaturated fatty acids in $\mathrm{m}$. soleus than sedentary rats. Whether the difference in lipid composition between trained and patients with T2DM is a consequence of the trained state or of dietary factors remains unknown. It has indeed recently been shown by TOF-SIMS that lipid composition of muscle fibers in mice can be affected by dietary lipid composition [25]. One should note, however, that despite the fact that the observed difference in double bound carbon groups was statistically significant, one may doubt if it this very modest difference is biologically meaningful.

Previous reports on LD morphology and location already indicated that these factors indeed may be involved in the athlete's paradox [14]. In this study [14], in which the groups were not matched for IMCL content, the highest lipid droplet number was also observed in type I fibers of the trained athletes. Another study, in which LD size was elegantly measured and was calibrated against phantoms, larger LDs were observed in type I fibers [13] and size was reported to correlate negatively with insulin sensitivity. In the current study we partly confirmed this negative association albeit in type II fibers only.

To study LD location, electron microscopy is most commonly being used. Electron microscopy provides accurate information about location, but information about fiber type is lacking. Immunofluorescence microscopy has the advantage that fiber type can be determined with parallel examinations of the SS and IMF region. Thus, we applied confocal immunofluorescence microscopy to measure LD morphology and location (based on LD-membrane distance) in a fiber type specific way and observed a positive association between SS LD number and insulin sensitivity in type I fibers. This matches observation of others [15-17]. Moreover, our data revealed that T2DM patients had larger LDs predominately in the SS region of type II fibers. Jointly, these data indicate that the most prominent difference in lipid storage pattern between Trained and T2DM patients are the large LDs in type II fibers which are mainly stored in the SS region whereas Trained store normal sized LDs mainly in type I fibers in the IMF region. So, we examined if the chemical composition of these LDs 
was different. Preliminary data on LD chemical lipid composition of large and small LDs in type II fibers of T2DM patients indeed revealed that large LDs in the SS region of type II fibers tend to have more saturated fat in the LD. These large LDs may interfere with insulin signaling since the turnover of fat in these large LDs may well be less dynamic and given the lower surface-to-volume ratio of large vs. small LDs and our previous observation that LD coat proteins -involved in creating access or docking-sites for lipases to the droplets are found at distinct spots on the LD, rather than surrounding the complete LD. A smaller surface-to-volume ratio results in less lipid droplet coating proteins and lipase access per lipid volume to control lipolysis [26], hence the control of regulated lipolysis may be less tight for large LDs, rendering these large LDs prone to incomplete lipolysis and elevated bioactive (insulin desensitizing) lipid moieties. In vitro models have shown that incubation of $\mathrm{C} 2 \mathrm{C} 12$ myotubes with saturated fatty acids induces insulin resistance, while this is not the case for incubation with the unsaturated fatty acid oleate [27]. It can be speculated that if these large LDs in the SS region of T2DM patients contain more saturated fatty acids and lipolysis is less well controlled, this might interfere with insulin signaling. In addition, having a LD distribution with most LDs being stored in the IMF fraction, like we observed in the endurance trained athletes, could be beneficial in the maintenance of insulin sensitivity. It has been observed by transmission electron microscopy and confocal fluorescence that LDs in the IMF region are always in close proximity of mitochondria $[28,29]$. This may indicate that for endurance trained athletes a larger fraction of the LDs is available to fuel mitochondria for muscle contractions. Endurance training in T2DM patients may induce a shift the IMCL storage pattern towards a phenotype like the athletes and thereby improving insulin sensitivity. It has been reported that endurance training in lean and obese participants reduces LD number in the SS region and increases LD number in the IMF region. [16] Furthermore, endurance training induces a fiber type shift towards a more oxidative phenotype [30]. Interestingly, the percentage of IMCL in contact with mitochondria increases in the IMF region with endurance training in lean and obese participants [16]. So, endurance training may result in a shift in the IMCL storage pattern as observed in T2DM patients towards a pattern that resembles the pattern observed in athletes (more LDs in the IMF region in mainly type I fibers) and thus contributing to training-mediated improvements in insulin sensitivity.

In conclusion, the main differences in the excess intramyocellular lipid storage of endurance trained athletes and T2DM are in the type II fibers. LD size and excess IMCL content in the SS region of type II fibers are important players in the athlete's paradox. If the subtle differences in number of unsaturated carbons in the LDs of type II fibers are of relevance for the athlete's paradox remains to be established. If the different LD composition is specific for large LDs and if indeed LD dynamics is 
compromised in these large LDs (hence rendering insulin resistance) will be a topic for further examination.

\section{REFERENCES}

1. Pan, D.A., et al., Skeletal muscle triglyceride levels are inversely related to insulin action. Diabetes, 1997. 46(6): p. 983-8.

2. Krssak, M., et al., Intramyocellular lipid concentrations are correlated with insulin sensitivity in humans: a 1 H NMR spectroscopy study. Diabetologia, 1999. 42(1): p. 113-6.

3. Goodpaster, B.H., et al., Intramuscular lipid content is increased in obesity and decreased by weight loss. Metabolism, 2000. 49(4): p. 467-72.

4. Goodpaster, B.H., et al., Skeletal muscle lipid content and insulin resistance: evidence for a paradox in endurance-trained athletes. J Clin Endocrinol Metab, 2001. 86(12): p. 5755-61.

5. Li, Y., et al., Protein kinase C Theta inhibits insulin signaling by phosphorylating IRS1 at Ser(1101). J Biol Chem, 2004. 279(44): p. 45304-7.

6. $\quad \mathrm{Yu}, \mathrm{C}$., et al., Mechanism by which fatty acids inhibit insulin activation of insulin receptor substrate-1 (IRS-1)-associated phosphatidylinositol 3-kinase activity in muscle. J Biol Chem, 2002. 277(52): p. 50230-6.

7. Chavez, J.A., et al., Ceramides and glucosylceramides are independent antagonists of insulin signaling. J Biol Chem, 2014. 289(2): p. 723-34.

8. Itani, S.I., et al., Lipid-induced insulin resistance in human muscle is associated with changes in diacylglycerol, protein kinase C, and IkappaB-alpha. Diabetes, 2002. 51(7): p. 2005-11.

9. Amati, F., et al., Skeletal muscle triglycerides, diacylglycerols, and ceramides in insulin resistance: another paradox in endurance-trained athletes? Diabetes, 2011. 60(10): p. 2588-97.

10. Bergman, B.C., et al., Increased intramuscular lipid synthesis and low saturation relate to insulin sensitivity in endurance-trained athletes. J Appl Physiol, 2010. 108(5): p. 1134-41.

11. Billecke, N., et al., Perilipin 5 mediated lipid droplet remodelling revealed by coherent Raman imaging. Integr Biol (Camb), 2015.

12. Koves, T.R., et al., PPARgamma coactivator-1alpha contributes to exercise-induced regulation of intramuscular lipid droplet programming in mice and humans. J Lipid Res, 2013. 54(2): p. 522-34.

13. He, J., B.H. Goodpaster, and D.E. Kelley, Effects of weight loss and physical activity on muscle lipid content and droplet size. Obes Res, 2004. 12(5): p. 761-9.

14. van Loon, L.J., et al., Intramyocellular lipid content in type 2 diabetes patients compared with overweight sedentary men and highly trained endurance athletes. Am J Physiol Endocrinol Metab, 2004. 287(3): p. E558-65.

15. Nielsen, J., et al., Increased subsarcolemmal lipids in type 2 diabetes: effect of training on localization of lipids, mitochondria, and glycogen in sedentary human skeletal muscle. Am J Physiol Endocrinol Metab, 2010. 298(3): p. E706-13.

16. Samjoo, I.A., et al., Markers of skeletal muscle mitochondrial function and lipid accumulation are moderately associated with the homeostasis model assessment index of insulin resistance in obese men. PLoS One, 2013. 8(6): p. e66322.

17. Li, Y., et al., Subsarcolemmal lipid droplet responses to a combined endurance and strength exercise intervention. Physiol Rep, 2014. 2(11).

18. Liu, Y., Y.J. Lee, and M.T. Cicerone, Broadband CARS spectral phase retrieval using a time-domain Kramers-Kronig transform. Opt Lett, 2009. 34(9): p. 1363-5. 
19. Camp, C.H., Y.J. Lee, and M.T. Cicerone, Quantitative, comparable coherent anti-Stokes Raman scattering (CARS) spectroscopy: correcting errors in phase retrieval. Journal of Raman Spectroscopy, 2015.

20. Wu, H., et al., In vivo lipidomics using single-cell Raman spectroscopy. Proc Natl Acad Sci U S A, 2011. 108(9): p. 3809-14.

21. Schneider, C.A., W.S. Rasband, and K.W. Eliceiri, NIH Image to ImageJ: 25 years of image analysis. Nat Methods, 2012. 9(7): p. 671-5.

22. Daemen, S., et al., Microscopy tools for the investigation of intracellular lipid storage and dynamics. Mol Metab, 2016. 5(3): p. 153-63.

23. Manco, M., et al., Insulin resistance directly correlates with increased saturated fatty acids in skeletal muscle triglycerides. Metabolism, 2000. 49(2): p. 220-4.

24. Nikolaidis, M.G., et al., Effect of chronic wheel running on the fatty acid composition of phospholipids and triacylglycerols in rat serum, skeletal muscle and heart. Acta Physiol Scand, 2004. 181(2): p. 199208.

25. Sjovall, P., et al., Dietary uptake of omega-3 fatty acids in mouse tissue studied by time-of-flight secondary ion mass spectrometry (TOF-SIMS). Anal Bioanal Chem, 2015. 407(17): p. 5101-11.

26. Hesselink, M.K., M. Mensink, and P. Schrauwen, Intramyocellular lipids and insulin sensitivity: does size really matter? Obes Res, 2004. 12(5): p. 741-2.

27. Chavez, J.A. and S.A. Summers, Characterizing the effects of saturated fatty acids on insulin signaling and ceramide and diacylglycerol accumulation in 3T3-L1 adipocytes and C2C12 myotubes. Arch Biochem Biophys, 2003. 419(2): p. 101-9.

28. Shaw, C.S., D.A. Jones, and A.J. Wagenmakers, Network distribution of mitochondria and lipid droplets in human muscle fibres. Histochem Cell Biol, 2008. 129(1): p. 65-72.

29. Vock, R., et al., Design of the oxygen and substrate pathways. VI. structural basis of intracellular substrate supply to mitochondria in muscle cells. J Exp Biol, 1996. 199(Pt 8): p. 1689-97.

30. Wilson, J.M., et al., The effects of endurance, strength, and power training on muscle fiber type shifting. J Strength Cond Res, 2012. 26(6): p. 1724-9. 


\section{CHAPTER 7}

\section{A microscopic view of intracellular cardiac lipid storage in humans}

Anne Gemmink, Gert Schaart, James P. Hobkirk, Matthijs K.C. Hesselink 


\section{CHAPTER 7}

\section{ABSTRACT}

Increased intracellular lipid content in cardiac muscle is observed in pathological conditions like ischemia and type 2 diabetes (T2DM). This can result in lipotoxic events which eventually can lead to cardiac dysfunction. In skeletal muscle rather differences in lipid droplet (LD) number and size are associated with insulin sensitivity than total lipid content. Here we aimed to examine LD size, number and location in coronary artery disease (CAD) patients with and without T2DM and patients without CAD (non-CAD) who underwent an aortic valve replacement.

Trucut needle biopsies of the left ventricle were taken during surgery of 6 non-CAD, 19 CAD and 7 CAD-T2DM patients. Biopsies were stained for laminin, desmin to stain the contractile elements and lipid droplets by Bodipy 493/503. Sections were imaged on a confocal microscope and quantified using ImageJ.

No significant differences were found for lipid area fraction, LD size and LD number. Though not significant, a step-wise increase in myocardial lipid content was observed with the highest levels in CAD-T2DM and the lowest in non-CAD (non-CAD: $0.26 \pm 0.10 \%, C A D: 0.45 \pm 0.10 \%$ and CAD-T2DM: $0.73 \pm 0.18 \% ; p=0.216$ ). This was accounted for by a stepwise increase LD number (non-CAD: $0.03 \pm 0.01 \# / \mu \mathrm{m}^{2}$, CAD: $0.04 \pm 0.01 \# / \mu \mathrm{m}^{2}$, CAD-T2DM: $0.07 \pm 0.01 \# / \mu \mathrm{m}^{2} ; p=0.256$ ). Myocardial lipid content, LD number and size did not associate with HOMA-IR. All LDs lied between the contractile elements. For both CAD groups, LD seems to be stored more nearby the T-tubuli than the non-CAD groups.

Myocardial lipid content in left ventricle tissue is determined by LD number, but not size. LD morphology of left ventricle tissue is not associated with insulin sensitivity. More LDs were located in the near vicinity of the t-tubular system in patients with CAD. If these factors are related to differences in cardiac function should be further examined. 


\section{INTRODUCTION}

Obesity and type 2 diabetes (T2DM) are major risk factors for cardiovascular diseases. Elevated levels of myocardial lipid content are observed in obesity [1] and diabetic cardiomyopathy [2]. Elevated cardiac lipid content can lead to lipotoxic events resulting in cardiac dysfunction [3]. Elevation in myocardial lipid storage may reflect an imbalance between fatty acid supply to and demand of the beating heart. Thus, myocardial fat content is often elevated in situations when the availability of plasma fatty acids is high. Under physiological conditions with high fatty acid availability, like after a 3-day very low calorie diet [4], fasting [5] and acute exercise in the fasted state [6], myocardial lipid storage is elevated. Whether or not the elevation of cardiac fat content translates into compromised cardiac function is still under debate. After 3 days of a very low calorie diet, for example, myocardial lipid content was elevated along with a declined left ventricular diastolic function [4]. Upon prolonged (3 hours) acute exercise in the fasted state, myocardial fat content increased in parallel with plasma free fatty acid levels [6]. Although this increase in myocardial lipid content after acute exercise reduced cardiac energy status the ejection fraction was not negatively affected. Under pathological conditions like T2DM [2] and ischemia $[7,8]$ increased levels of myocardial lipid content is observed, which in patients with type 2 diabetes or impaired glucose tolerance is accompanied by a declined left ventricular diastolic function [9].

Conversely, studies designed to reduce myocardial lipid content e.g., by a very low calorie diet for 16 weeks resulted in a reduction of cardiac lipid content in T2DM patients with a simultaneous improvement of diastolic cardiac function [10]. The addition of an exercise program on top of the dietary intervention, however, did not result in an additional reduction of cardiac lipid content nor did it improve cardiac function. In a 12-week exercise intervention study in patients with type 2 diabetes, systolic cardiac function improved while myocardial lipid content remained unaffected [11]. These data, largely based on non-invasive NMR spectroscopy in a voxel positioned in the septum of the beating heart, indicate that the link between myocardial fat storage and compromised cardiac function is not straightforward and mimics the situation observed in skeletal muscle.

In insulin resistant subjects, intramyocellular lipid (IMCL) content correlates negatively with peripheral insulin sensitivity [12-14]. In trained subjects, however, this relationship is absent [15]. Trained athletes possess high MCL levels whilst being rather insulin sensitive. Moreover, exercise training can profoundly improve peripheral insulin sensitivity without affecting total IMCL content $[16,17]$. However, IMCL content is the total product of lipid droplet (LD) size and number. He et al. 2004 [16] showed that upon exercise training LD size decreased which was associated with the 
improvement in peripheral insulin sensitivity. We (chapter 6) have shown that patients with type 2 diabetes differ from athletes by preferential storage of lipids in large droplets in type II muscle fibers predominantly in the subsarcolemmal region, whereas trained athletes store their lipids predominantly in small but numerous lipid droplets in the intramyofbrillar space in type I muscle fibers. This indicates that not only the total lipid content but also the morphometric characteristics and subcellular distribution of lipids may affect muscle cell function. However, data on cardiac LD morphology and subcellular distribution in relation to cardiac function in humans is scarce. It has been reported that obese patients with dilated cardiomyopathy have a higher number of lipid droplets than lean patients [18], data on size, however, were not reported. An elegant 3D electron microscopy reconstruction of a healthy heart donor revealed that LDs are localized in the near vicinity of T-tubuli and mitochondria [19], underscoring the suggestion that like in skeletal muscle, also in the heart the subcellular distribution of lipid droplets may be of relevance.

In the present study we aimed to examine LD size, number and location in human left ventricle biopsies in relation to disease state. To this end we compared biopsies from the human left ventricle from patients without known disturbances in glucose metabolism whom had to undergo cardiac surgery for an aortic valve replacement (non-CAD) with biopsies from patients with diagnosed coronary artery disease who were normoglycemic (CAD) or patients with diagnosed cardiac artery disease who were diagnosed with type 2 diabetes (CAD-T2DM). In these groups we examined cardiac lipid content, lipid droplet number and lipid droplets size. Moreover, we examined the subcellular distribution of cardiac lipid droplets under these conditions.

\section{METHODS}

\section{Subjects}

Thirty-two subjects who underwent an open heart surgery participated in this study. Twenty-six subjects had coronary artery disease (CAD) and underwent a coronary artery bypass graft. Subjects undergoing coronary artery bypass graft were divided into two groups, 9 subjects with T2DM (CADT2DM) and 17 subjects without T2DM (CAD). Six subjects underwent an aortic valve replacement and did not have cardiovascular diseases and T2DM (non-CAD). The non-CAD served as a control group. During cardiac surgery two left ventricular trucut needle biopsies were taken. Biopsies were snap frozen in melting isopentane and stored at $-80^{\circ} \mathrm{C}$ until histochemical analyses. Blood plasma was analyzed for glucose, insulin, total cholesterol, LDL cholesterol, HDL cholesterol and triglycerides. The HOMA index was calculated from the fasting plasma glucose and insulin values. 


\section{Histochemical analysis}

Five $\mu \mathrm{m}$ thick sections were cut and mounted on glass slides. Each glass slide contained a subject from the three different groups to minimize bias originating from putative variation in staining intensity. The whole procedure (staining, imaging and quantification) was performed blinded with regard disease state. In addition, as an internal control for staining quality a skeletal muscle sample from an endurance trained athlete (always the same biopsy) was mounted as well on every single glass slide. Sections were fixated for 30 minutes with $3.7 \%$ formaldehyde/PBS. After rinsing, sections were blocked with blocking buffer ( $150 \mathrm{mM} \mathrm{NaCl}, 20 \mathrm{mM}$ Tris pH 6.8 and 2\% BSA) for 45 minutes. Subsequently, sections were permeabilized with $0.25 \%$ Triton TX-100/PBS. Sections were rinsed for 5 minutes with PBS and incubated for 1 hour with primary antibodies against laminin (L9393, Sigma, MO, USA) and desmin (sc-58745, Santa Cruz, CA, USA) both diluted 1:25 in 0.05\% Tween/PBS. Subsequently, Bodipy 493/503 (D3922, Molecular Probes, Leiden, The Netherlands) and appropriate secondary antibodies conjungated with AlexaFluor-405 (AF405) and AlexaFluor-647 (AF647) were incubated for 90 minutes at $37^{\circ} \mathrm{C}$. Sections were mounted in Mowiol and covered with coverslips \#1. Until imaging, sections were stored in the dark at room temperature.

Sections were imaged on a Leica TCS SP8 confocal microscope with a 100x 1.4 N.A. oil immersion objective. Images contained $2048 \times 2048$ pixels and 4 slices with $0.17 \mu \mathrm{m}$ in between for deconvolution purposes. For measuring lipid area fraction, LD size and number a 1.6x optical zoom was used resulting in a pixel size of $35.5 \mathrm{~nm} \times 35.5 \mathrm{~nm}$. For imaging subcellular distribution of LDs (in direct vicinity of the t-tubular system or in in contact with contractile elements) a $5 x$ optical zoom was used. This resulted in a pixel size of $11.4 \mathrm{~nm} \times 11.4 \mathrm{~nm}$. For imaging laminin-AF405 the 405 laser line was used. For imaging Bodipy 493/503 and desmin-AF647 the white laser line was used. For imaging Bodipy 493/503 a wavelength of $488 \mathrm{~nm}$ and for desmin-AF647 a wavelength of $647 \mathrm{~nm}$ were used for excitation. All fluorophores were detected with a photomultiplier tube. Emission was detected between $415 \mathrm{~nm}-460 \mathrm{~nm}, 500 \mathrm{~nm}-530 \mathrm{~nm}$ and $657 \mathrm{~nm}-750 \mathrm{~nm}$ for respectively laminin-AF405, Bodipy 493/503 and desmin-AF647. Acquired images were deconvoluted with Huygens Professional software (Scientific Volume Imaging B.V., Hilversum, The Netherlands) to improve signal-to-noise ratio. Total fat content measured as lipid area fraction, LD size and number were measured with a custom written macro in ImageJ [20]. 


\section{Statistics}

Data are presented as mean \pm SEM. To test for statistical significant differences among groups a Oneway ANOVA was used. If a significant group effect was found a Bonferroni post-hoc test was performed. A $p<0.05$ was considered as statistical significantly different. Pearson correlation coefficients were determined in case an association between the LD parameters and the HOMA index was observed.

\section{RESULTS}

\section{Subject characteristics and blood plasma parameters}

Although the total cohort comprises 32 subjects, freezing artifacts in the left ventricle biopsies of 5 subjects precluded these biopsies for further analysis. Data of the drop-outs for histological analyses were disregarded for all analysis and hence are not included in the plasma data either. Thus, a total of 27 subjects (5 non-CAD, 14 CAD and 8 CAD-T2DM) were analyzed for intramyocardial lipid content, LD morphology and subcellular distribution of LD.

Table 1. Subject characteristics and blood plasma parameters

\begin{tabular}{llll}
\hline Parameter & non-CAD & CAD & CAD-T2DM \\
\hline Gender (F/M) & $3 / 2$ & $4 / 10$ & $2 / 6$ \\
Age (years) & $73 \pm 4$ & $66 \pm 2$ & $62 \pm 4$ \\
Weight $(\mathrm{kg})$ & $64.5 \pm 5.4$ & $89.2 \pm 4.2$ & $101.6 \pm 5.7$ \\
Height $(\mathrm{m})$ & $1.57 \pm 0.02$ & $1.69 \pm 0.03$ & $1.73 \pm 0.04$ \\
BMI $\left(\mathrm{kg} / \mathrm{m}^{2}\right.$ ) & $26 \pm 2$ & $31 \pm 2$ & $34 \pm 2$ \\
Fasting plasma glucose (mmol/L) & $5.86 \pm 0.20$ & $5.77 \pm 0.13$ & $7.93 \pm 0.56^{* \#}$ \\
Insulin (ulU/ml) & $5.13 \pm 2.00$ & $4.83 \pm 0.97$ & $11.82 \pm 2.61^{\#}$ \\
HOMA-index & $1.33 \pm 0.50$ & $1.26 \pm 0.22$ & $4.03 \pm 0.77^{* \#}$ \\
Total cholesterol (mmol/L) & $3.63 \pm 0.26$ & $3.32 \pm 0.17$ & $3.41 \pm 0.32$ \\
LDL cholesterol (mmol/L) & $1.88 \pm 0.27$ & $1.72 \pm 0.10$ & $1.72 \pm 0.24$ \\
HDL cholesterol (mmol/L) & $1.20 \pm 0.11$ & $0.97 \pm 0.06$ & $0.76 \pm 0.06^{*}$ \\
Triglycerides (mmol/L) & $1.19 \pm 0.18$ & $1.38 \pm 0.25$ & $2.05 \pm 0.33$ \\
\hline${ }^{*} p<0.05$ vs. non-CAD; $\# p<0.05$ vs. CAD & & &
\end{tabular}

Subject characteristics and blood plasma parameters are presented in Table 1. Age, weight, height and BMI did not differ among groups. CAD-T2DM had significantly higher fasting plasma glucose than CAD and non-CAD $(p<0.001)$ and higher plasma insulin levels than CAD $(p<0.05)$. HOMA-index was used as a surrogate marker for insulin sensitivity and was significantly higher in CAD-T2DM than the other groups $(p<0.001)$. Total cholesterol, LDL cholesterol and triglyceride levels did not differ 
among groups. For blood plasma HDL differences among groups were observed. HDL was lower in CAD-T2DM patients compared to the non-CAD group $(p<0.01)$. All subjects were on statin therapy.

\section{Intramyocardial lipid storage}

Total myocardial lipid content was the lowest in non-CAD and highest in the CAD-T2DM group (Figure $1 \mathrm{~A}$ and $1 \mathrm{~B})$, but these differences were not significantly different $(0.26 \pm 0.10 \%, 0.45 \pm 0.11 \%$ and $0.65 \pm 0.15 \%$ for respectively non-CAD, CAD and CAD-T2DM; $p=0.216)$. The stepwise increase in total myocardial lipid content from non-CAD to CAD-T2DM was accounted for by a stepwise increase in the number of LDs (Figure 1A and 1C; $0.03 \pm 0.01 \# / \mu \mathrm{m}^{2}, 0.04 \pm 0.01 \# / \mu \mathrm{m}^{2}, 0.06 \pm 0.01 \# / \mu \mathrm{m}^{2}$ for respectively non-CAD, CAD and CAD-T2DM; $p=0.256)$ rather than in size $\left(0.096 \pm 0.005 \mu \mathrm{m}^{2}\right.$, $0.102 \pm 0.004 \mu \mathrm{m}^{2}, 0.103 \pm 0.005 \mu \mathrm{m}^{2}$ for respectively non-CAD, CAD and CAD-T2DM; $p=0.678$; Figure 1D). Total intramyocardial lipid content and LD number show a remarkably large variation in all groups. Upon examination of all subjects myocardial lipid content and LD morphology were not associated with the HOMA index (myocardial lipid content: $r=0.061, p=0.763$; LD number: $r=0.086$, $p=0.670$; LD size: $r=-0.009, p=0.965$ ).

A

non-CAD

CAD

CAD-T2DM
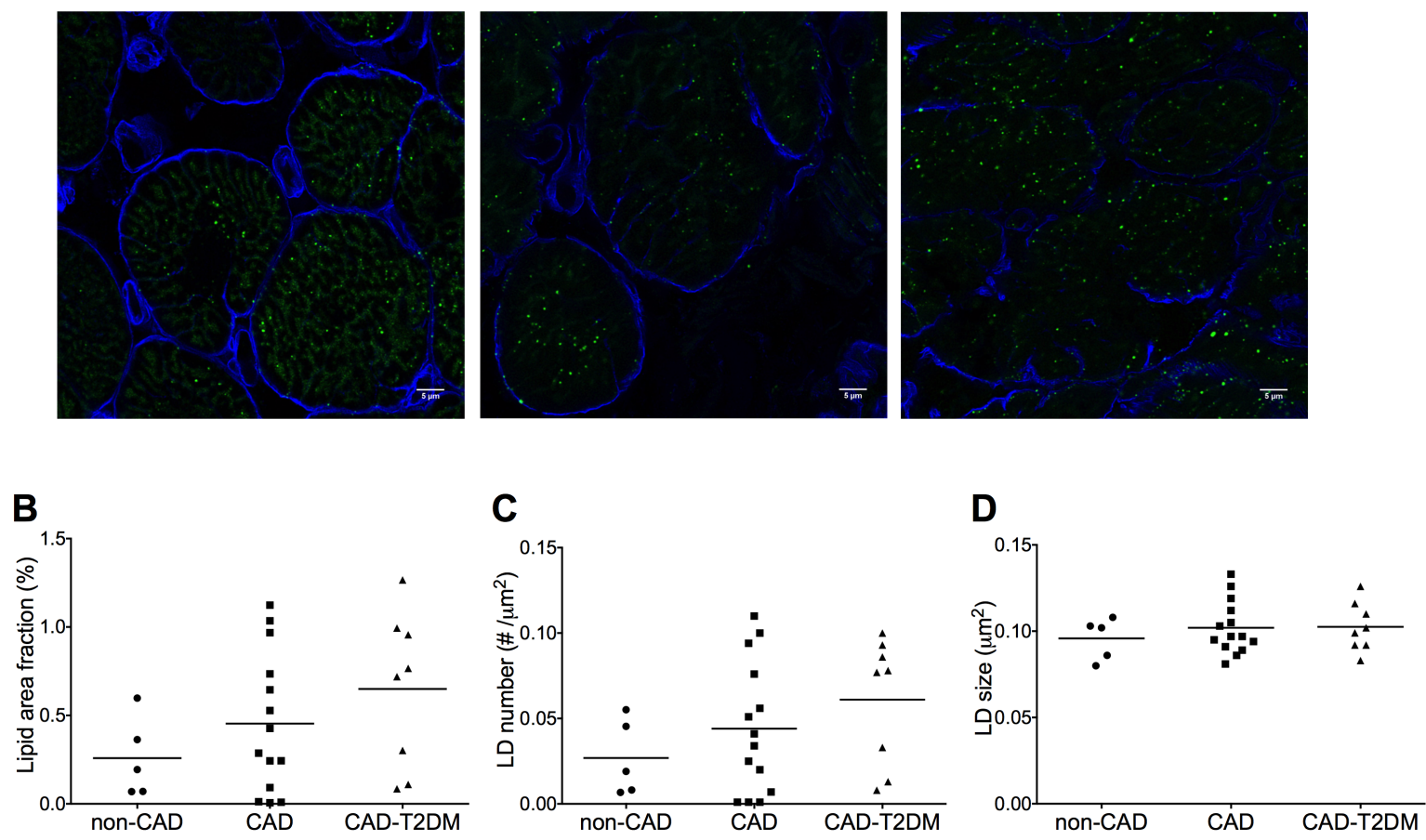

Figure 1. Morphology of intracellular cardiac lipid storage. (A) Representative confocal immunofluorescent images of cardiac lipid storage of non-CAD, CAD and CAD-T2DM. (B) Quantification of lipid area fraction, (C) lipid droplet number and (D) lipid droplet size. 


\section{Lipid droplet location}

In human skeletal muscle LD location within the cell is related to insulin sensitivity [21]. We thus examined LD location within cardiomyocytes in a descriptive manner. LD location was examined in cardiomyocytes sectioned cross-sectionally as well as and longitudinally. In cardiomyocytes cut cross-sectionally, LD location was studied in relation to the t-tubular system whereas in cardiomyocytes cut longitudinally, LD location was studied relative to the Z-lines (as indicated by staining of the Z-line interconnecting intermediate filament desmin).

In the cross-sectionally cut cardiomyocytes LDs in the CAD groups were more prominent in near vicinity of the t-tubules, whereas in the non-CAD group, LDs were dispersed throughout the cardiomyocytes (Figure $2 \mathrm{~A}-\mathrm{C}$ ). In longitudinally cut cardiomyocytes LDs were located between the contractile elements (Figure 2D-F).
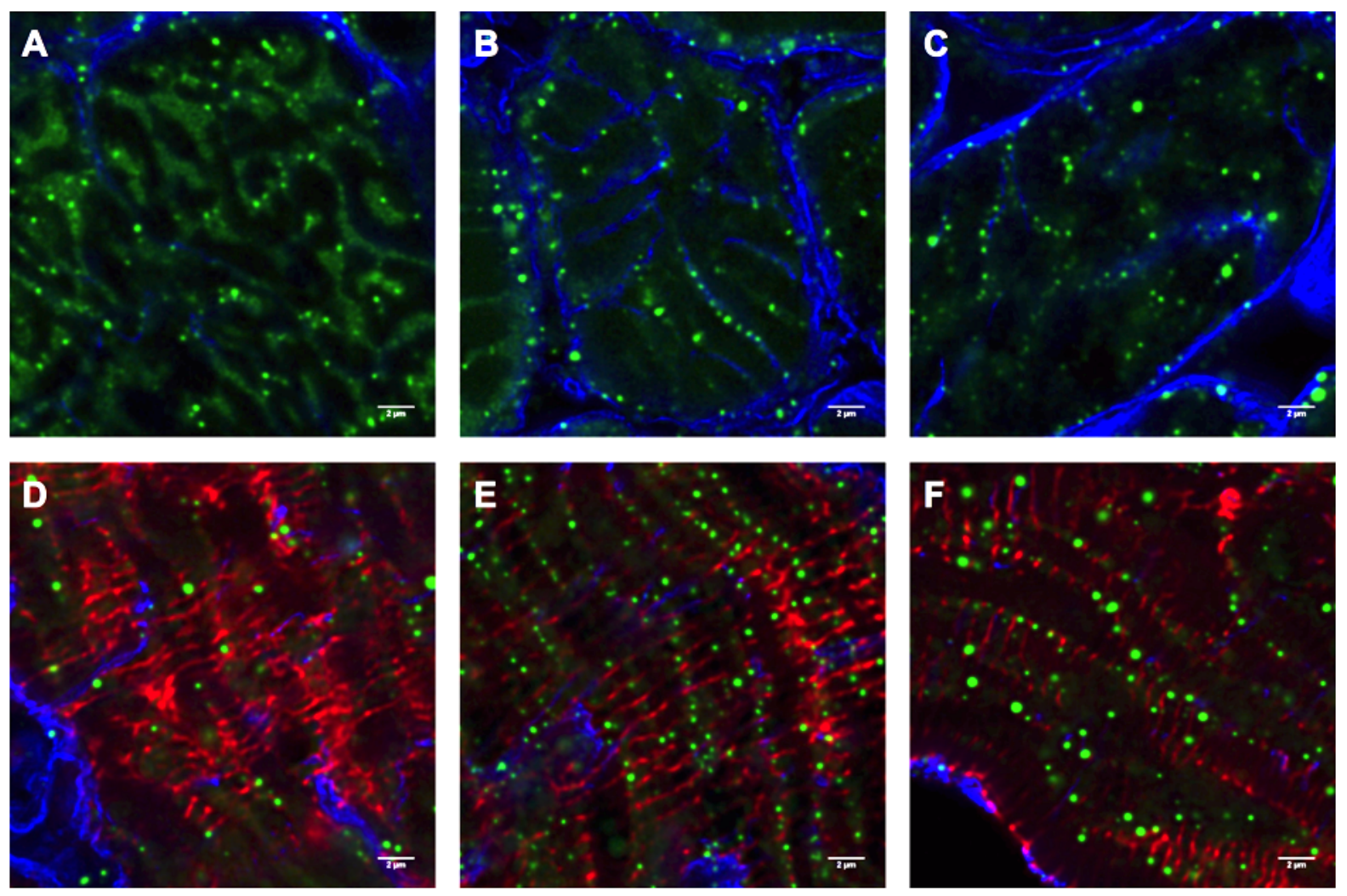

Figure 2. Confocal immunofluorescence images of LD location in reference to T-tubuli and Z-lines (as indicated by staining of the Z-line interconnecting intermediate filament desmin). LDs (green) and T-tubuli (blue) of (A) non-CAD, (B) CAD and (C) CAD-T2DM. Both CAD groups store LDs in the vicinity of the T-tubuli. LDs (green), Ttubuli (blue) and desmin (red) of (D) non-CAD, (E) CAD and (F) CAD-T2DM. LDs are located between the contractile elements in all groups. 


\section{DISCUSSION}

In this study we aimed to examine LD morphology and location in human left ventricular biopsies. We studied LD morphology and location in coronary artery disease (CAD) patients with and without T2DM, and metabolically non-compromised subjects who needed an aortic valve replacement as a control group. Although the cardiac fat content displays a stepwise increase with increased metabolic disease sate (least cardiac muscle fat in non-CAD, more in CAD and most in CAD-T2DM), these differences failed to reach significance. The stepwise increase in cardiac fat content most likely originates from an elevated number of LDs rather than LD size. Furthermore, LD morphology and myocardial lipid content were not associated with compromised glucose handling, as assessed by the HOMA-index. In addition, LD location within cardiomyocytes appears to differ slightly amongst groups. In subjects undergoing an aortic valve replacement (AVR), LDs were more dispersed throughout the cell while in both CAD groups LDs were located in the vicinity of the t-tubules. LDs dispersed between the contractile elements were similarly observed in all groups.

Studies examining human left ventricular lipid content by microscopy are scarce and features of LDs which are associated with lipotoxic processes in skeletal muscle (like LD size, number and location) are understudied in the human heart. The limited studies thus far, are generally performed in left ventricle samples from autopsies or transplanted hearts $[2,22]$ or from biopsies obtained during open heart surgery [23] and have not been used for a more detailed intramyocardial lipid analysis by high resolution microscopy $[2,22,23]$. Previous studies reported elevated lipid content in the left ventricle of type 2 diabetes patients [2, 22] and subjects with metabolic syndrome [23] in association with declined ejection fraction [23]. Furthermore, obese heart failure patients and heart failure patients with type 2 diabetes display higher levels of intramyocardial lipid content than lean heart failing patients [2], suggesting an association between cardiac lipid content and cardiac function. In an autopsy based study, Nakanishi et al. 2014 [22] intended to study cardiac lipid storage in left ventricle tissue. Interestingly, in 73 autopsies, lipid droplets were only detectable in the heart of 7 subjects, all of whom were diagnosed patients with type 2 diabetes. On the other hand, not all type 2 diabetes patients included in this study $(n=14)$ displayed elevated cardiac lipid storage. This is in accordance with our data were we report a large variation in intramyocardial lipid content in all groups and no association between myocardial lipid content and insulin sensitivity. Moreover, both groups with CAD possessed elevated intramyocardial lipid content. Although the number of subjects in the present study was relatively limited, this suggests that type 2 diabetes per se does not necessarily associate with higher myocardial lipid storage. 
Total intramyocardial lipid content is the product of LD size and number. Human and rodent studies examining the number and size of cardiac lipid droplets are scarce. Upon overexpression of PPARY, the number as well as the size of cardiac lipid droplets increases, with increased cardiac fat content as a consequence. Under these none-physiological conditions, the increase in lipid droplet size in the heart was paralleled by development of dilated cardiomyopathy and premature death as a consequence of ventricular fibrillation [24]. In more physiological settings, when mice were fasted for 48 hours, the number of lipid droplets increased without effects on lipid droplet size [5], an observation which mimics our current observations of increased cardiac lipid content originating from increased lipid droplet number rather than size. The only human study we could trace on this topic reports a higher number of LDs in obese subjects with dilated cardiomyopathy compared to their lean counterparts [18], LD size, however, was not assessed. Jointly, these data may indicate that in the early stages of excess myocardial fat storage, lipid droplets increase in number and that an increase in size is warranted to exert negative effects on cardiac function.

While studies on lipid droplet size and number in human hearts are scarce, studies focusing on subcellular distribution of lipid droplets in the heart are virtually non-existing. In skeletal muscle, storage of lipid droplets in vicinity of the sarcolemma has been associated with insulin resistance [21]. Just like skeletal muscle, cardiomyocytes have invaginations of the sarcolemma towards the core of the muscle fibers, a system referred to as the t-tubular system. The t-tubular system is essential for a rapid and spatially simultaneous propagation of the cardiac action potential throughout the cardiac muscle fiber. The connectivity of the t-tubular system with the calcium containing sarcoplasmic reticulum facilitates swift entry (and reuptake) of $\mathrm{Ca}^{2+}$ to and from the cardiomyocyte with a homogenous cardiac myocyte contraction as the functional outcome [25]. Whether or not the subcellular distribution of cardiac LDs plays a role in cardiac dysfunction is presently unknown. One human study examined the location of cardiac LDs relative to the t-tubules in a non-failing donor heart [19]. This study reported that $29 \%$ percent of the LDs were located in the subsarcolemmal region. Of the remaining intermyofibrillar LDs, $59 \%$ was within a distance of 0.5 $\mu \mathrm{m}$ from a t-tubule. In the present study we observed in the CAD groups that LDs were located near the t-tubules whereas in the non-CAD group LDs were more dispersed throughout the cardiomyocyte. With the current data we can only speculate if the subcellular location of lipid droplets indeed affects cardiac function. It is, however, not unlikely that lipid droplets in vicinity of the t-tubular system interfere with propagation of the action potential and hence compromise cardiac contractility. 
In summary, intramyocardial lipid content was not significantly different between patients with coronary artery disease with and without type 2 diabetes, and patients without coronary artery disease. Cardiac lipid content seems to be dominated by LD number rather than LD size. In addition, LDs in patients with CAD were located in vicinity of the t-tubular system, an observation that was not made in the metabolically non-compromised valve replacement patients. If this difference in subcellular location indeed impacts for cardiac function requires further examination.

\section{REFERENCES}

1. Szczepaniak, L.S., et al., Myocardial triglycerides and systolic function in humans: in vivo evaluation by localized proton spectroscopy and cardiac imaging. Magn Reson Med, 2003. 49(3): p. 417-23.

2. Sharma, S., et al., Intramyocardial lipid accumulation in the failing human heart resembles the lipotoxic rat heart. FASEB J, 2004. 18(14): p. 1692-700.

3. D'Souza, K., C. Nzirorera, and P.C. Kienesberger, Lipid metabolism and signaling in cardiac lipotoxicity. Biochim Biophys Acta, 2016.

4. Hammer, S., et al., Short-term flexibility of myocardial triglycerides and diastolic function in patients with type 2 diabetes mellitus. Am J Physiol Endocrinol Metab, 2008. 295(3): p. E714-8.

5. Suzuki, J., et al., Cardiac gene expression profile and lipid accumulation in response to starvation. Am J Physiol Endocrinol Metab, 2002. 283(1): p. E94-E102.

6. Bilet, L., et al., Exercise-induced modulation of cardiac lipid content in healthy lean young men. Basic Res Cardiol, 2011. 106(2): p. 307-15.

7. Jodalen, $\mathrm{H}$., et al., Lipid accumulation in the myocardium during acute regional ischaemia in cats. J Mol Cell Cardiol, 1985. 17(10): p. 973-80.

8. Bilheimer, D.W., et al., Fatty acid accumulation and abnormal lipid deposition in peripheral and border zones of experimental myocardial infarcts. J Nucl Med, 1978. 19(3): p. 276-83.

9. McGavock, J.M., et al., Cardiac steatosis in diabetes mellitus: a 1H-magnetic resonance spectroscopy study. Circulation, 2007. 116(10): p. 1170-5.

10. Hammer, S., et al., Prolonged caloric restriction in obese patients with type 2 diabetes mellitus decreases myocardial triglyceride content and improves myocardial function. J Am Coll Cardiol, 2008. 52(12): p. 1006-12.

11. Schrauwen-Hinderling, V.B., et al., Cardiac lipid content is unresponsive to a physical activity training intervention in type 2 diabetic patients, despite improved ejection fraction. Cardiovasc Diabetol, 2011. 10: p. 47.

12. Krssak, M., et al., Intramyocellular lipid concentrations are correlated with insulin sensitivity in humans: a $1 H$ NMR spectroscopy study. Diabetologia, 1999. 42(1): p. 113-6.

13. Pan, D.A., et al., Skeletal muscle triglyceride levels are inversely related to insulin action. Diabetes, 1997. 46(6): p. 983-8.

14. Goodpaster, B.H., et al., Intramuscular lipid content is increased in obesity and decreased by weight loss. Metabolism, 2000. 49(4): p. 467-72.

15. Goodpaster, B.H., et al., Skeletal muscle lipid content and insulin resistance: evidence for a paradox in endurance-trained athletes. J Clin Endocrinol Metab, 2001. 86(12): p. 5755-61.

16. He, J., B.H. Goodpaster, and D.E. Kelley, Effects of weight loss and physical activity on muscle lipid content and droplet size. Obes Res, 2004. 12(5): p. 761-9. 
17. Meex, R.C., et al., Restoration of muscle mitochondrial function and metabolic flexibility in type 2 diabetes by exercise training is paralleled by increased myocellular fat storage and improved insulin sensitivity. Diabetes, 2010. 59(3): p. 572-9.

18. Saito, T., et al., Myocardial alterations and clinical implications associated with recovery of cardiac function in dilated cardiomyopathy with obesity. Int J Cardiol, 2013. 168(1): p. 144-50.

19. Sulkin, M.S., et al., Nanoscale three-dimensional imaging of the human myocyte. J Struct Biol, 2014. 188(1): p. 55-60.

20. Schneider, C.A., W.S. Rasband, and K.W. Eliceiri, NIH Image to ImageJ: 25 years of image analysis. Nat Methods, 2012. 9(7): p. 671-5.

21. Nielsen, J., et al., Increased subsarcolemmal lipids in type 2 diabetes: effect of training on localization of lipids, mitochondria, and glycogen in sedentary human skeletal muscle. Am J Physiol Endocrinol Metab, 2010. 298(3): p. E706-13.

22. Nakanishi, T. and S. Kato, Impact of diabetes mellitus on myocardial lipid deposition: an autopsy study. Pathol Res Pract, 2014. 210(12): p. 1018-25.

23. Marfella, R., et al., Myocardial lipid accumulation in patients with pressure-overloaded heart and metabolic syndrome. J Lipid Res, 2009. 50(11): p. 2314-23.

24. Liu, L., et al., Diacylglycerol acyl transferase 1 overexpression detoxifies cardiac lipids in PPARgamma transgenic mice. J Lipid Res, 2012. 53(8): p. 1482-92.

25. Brette, F. and C. Orchard, T-tubule function in mammalian cardiac myocytes. Circ Res, 2003. 92(11): p. 1182-92. 


\section{CHAPTER 8}

General Discussion 
In this thesis lipid droplet (LD) characteristics in relation to insulin sensitivity in human skeletal muscle were studied by using mainly a microscopic approach. Since high levels of IMCL do not always associate with insulin resistance, the so-called athlete's paradox [1], LD characteristics are putatively a more important determinant for insulin sensitivity than total intramyocellular lipid (IMCL) content. For examining LD characteristics like size, number and location, microscopy is a commonly used tool. However, for LD features like decoration, dynamics and lipid composition in skeletal muscle protein and lipid extractions are used in general. Unlike microscopy, however, these approaches do not provide insight into how the LD is decorated, at which subcellular site interaction between proteins involved in LD dynamics occurs and if the lipid composition of LDs is different between LDs and more particularly if these putative differences help to explain the athlete's paradox.

\section{IMAGING OF INTRAMYOCELLULAR LIPID STORAGE PATTERN}

The relationship between the total IMCL pool and insulin sensitivity is not straightforward [1-6]. Several studies have therefore focused on the LD morphology and location. To study these LD features, both fluorescence and electron microscopy are commonly used techniques. Endurance trained athletes and type 2 diabetes patients matched for total IMCL content possess a different IMCL storage pattern; with a higher LD number in type I fibers in athletes and larger LDs in type II fibers in type 2 diabetes patients. In addition, type 2 diabetes patients store these large LDs in a high number in type II fibers mainly in the subsarcolemmal (SS) region (Chapter 6). However, He et al. 2004 [7] observed in type I fibers larger LDs in type 2 diabetes patients compared to lean subjects. This different pattern is in accordance with the effect of exercise interventions resulting in a reduction of IMCL content [8], LD size [9, 10] and LD number [9-11] in the SS region has in the past been measured with electron microscopy (EM). However, if this is fiber type specific or not is unknown, as making the distinction between the different fiber types is typically not straightforward with EM. Jointly, this indicates that high lipid content in the SS region is a consequence of largely sized and numerous LDs in one of the fiber types is non-beneficial for peripheral insulin sensitivity. IMCL storage in the near vicinity of the cell membrane can putatively interfere with local insulin signaling. However, basic questions like where are LDs formed in skeletal muscle (in the SS region or in the IMF region) and why high levels of IMCL are stored in the SS regions in pathological insulin resistance remains elusive. In cell models it has been reported that upon starvation LDs become mobile and move throughout the cells via the microtubular system [12]. In addition, the LDmitochondria interaction is stimulated as well. So, potentially the LD movement in insulin resistance 
is disturbed resulting in high IMCL levels in the SS fraction. If this LD movement is disturbed in skeletal muscle of type 2 diabetes patients requires additional elaboration e.g. using life-cell imaging in cultured primary myotubes obtained from T2DM patients.

All studies examining IMCL storage pattern in human skeletal muscle use fluorescence or electron microscopy. Both techniques have their drawbacks, but combined this could be a powerful tool to study IMCL content. With fluorescence microscopy a large field of view can be studied and different fiber types can be distinguished, while electron microscopy has a high spatial resolution, provides ultrastructural information and is able to accurately differentiate between the SS and intermyofibrillar (IMF) regions, but little information of the fiber type under examination is provided. When these two techniques are combined like correlative light and electron microscopy (CLEM), fiber type specific measurements can be performed for SS and IMF LDs. CLEM can be performed in several ways, by integrative microscope systems, by software matching coordinates of images [13, 14] or by aligning fluorescence and electron microscopy images based upon on subcellular landmarks $[14,15]$. The advantage of an integrative system is a perfect overlay of the images, while the unique features of the latter two super-resolution fluorescence microscopy technologies can be used like STED or PALM [13, 15]. Differences between endurance trained athletes and type 2 diabetes patients were mainly found in the SS region of type II fibers (Chapter 6). CLEM combined with super-resolution fluorescence microscopy can give more insight if these SS LDs are different than IMF LDs in regard with protein coating, which could be of importance for the LD dynamics.

\section{MICROSCOPY, A USEFUL TOOL TO STUDY DYNAMICS OF INTRAMYOCELLULAR LIPID DROPLETS}

Lipid droplets are nowadays recognized as dynamic organelles. Lipid storage as a result of large LDs might be an indication of LDs with compromised dynamics. Important players in intramyocellular LD dynamics are ATGL, HSL, CGI-58, GOS2 and PLIN2, and PLIN5. Most well-studied proteins in intramyocellular LD dynamics are ATGL (main triacylglycerol lipase), CGI-58 (co-activator of ATGL) and PLIN5. PLIN5 regulates LD lipolysis by interacting with CGI-58 during high availability of longchain fatty acids. LD lipolysis is stimulated when CGI-58 is released from PLIN5 and colocalizes with ATGL [16]. High levels of PLIN5 protein are observed in insulin sensitive endurance trained athletes $[17,18]$ (Chapter 5). However, the relationship between PLIN5 protein content and insulin sensitivity is not conclusive. Koves et al. 2013 [18] showed that maximal oxidative capacity and PLIN5 are predictors for insulin sensitivity. Although, when performing a stepwise regression we observed that 
maximal oxidative capacity is the main predictor of insulin sensitivity and PLIN5 is only a predictor when maximal oxidative capacity is taken into account (Chapter 5). On the other hand, PLIN5 protein content is positively associated with insulin sensitivity in lean subjects with a Caucasian, but not of South Asian origin (Chapter 2). Putatively, PLIN5 is only protective against insulin sensitivity when other, yet to be identified, conditions are met. One such condition we proved to be required is a high oxidative capacity (Chapter 5 ). Other conditions that might be met are the interaction with other proteins involved in regulating lipolysis, like ATGL and CGI-58.

In human skeletal muscle, important players in LD dynamics [18-20] (Chapter 2) have been examined. Our results appear to indicate that some balance between releasing and storing fatty acids from/in the LD is required to maintain cellular health. Total protein content, however, does not provide insight into the dynamic processes involved in LD generation and maintenance. Rather, the interaction with other proteins and the location of these proteins will give additional insights in how dynamic the LD is or can be. The presence of PLIN5 is known not to be restricted to the LD, but it is also reported to be present in mitochondria [21-24] and in the cytosolic fraction [25, 26]. For more close examination of the location of this protein along with its orientation around other organelles CLSM can be a useful tool. By using CLSM we observed that upon 60 hours fasting a higher fraction of PLIN5 was associated with LDs despite no change in total PLIN5 protein content (Chapter 4). In addition, by characterizing LDs by their coating with PLIN5 we observed that upon prolonged fasting PLIN5 coated LDs were protective against insulin resistance and LDs devoid of PLIN5 were not (Chapter 4). In a cross-sectional comparison PLIN5 coated LDs are not directly related with insulin sensitivity, but only in combination with a high maximal oxidative capacity (Chapter 5 ). The coating of a LD with PLIN5 may well be an indication that it is a dynamic LD since PLIN5 is regulating LD lipolysis by interacting with CGI-58 [16]. However, coating of a LD with PLIN5 per se is still not a direct reflection of its dynamics, since no interaction with other (e.g., lipolytic) proteins is measured. This could explain the non-conclusive results about the association between PLIN5 coated LDs and insulin sensitivity in this thesis. In skeletal muscle protein interaction of important players of LD dynamics are examined with immunoprecipitation $[27,28]$ or by conventional fluorescence microscopy [29]. By using immunoprecipitation interaction between proteins can be measured. However, the location of this interaction is unknown, which can be of functional relevance. When using fluorescence microscopy for determining interaction between proteins a high resolution is needed. In order to prevent an overestimation of protein colocalization it is of importance that out of focus light is limited. For a long time CLSM has been applied in studies towards protein colocalization. However, in the meantime super-resolution techniques are developed to overcome the optical diffraction limit of light microscopy. This advance in the field yields a more powerful type 
of microscopy for colocalization studies by imaging. One of these techniques is Stimulated Emission Depletion (STED) microscopy. STED is confocal microscopy with an addition of a depletion laser and it overcomes the optical diffraction limit by actively depleting some of the emitted light. With the optimal settings a resolution of $60 \mathrm{~nm}$ can be reached [30-32]. In chapter 3 we show that we successfully set up two-color STED imaging and with proper settings the proteins on the inner and outer mitochondrial membrane can be distinguished. An advantage of STED imaging over other super-resolution techniques, like STORM and PALM, is that STED is more suitable for live-cell imaging [30]. STED microscopy could therefore be a useful tool to study LD dynamics in primary myotubes. In addition, STED microscopy can give insights in LD dynamics of skeletal muscle upon acute exercise and lipid infusions studies in groups with different levels of insulin sensitivity. These types of studies will challenge the LDs and depending on differences in LD dynamics insulin sensitivity, IMCL content, and/or LD morphology is affected differently. Subsequently, live cell imaging of cultured human primary myotubes from donors with different phenotypes challenged with starvation, oversupply of fatty acids or stimulated lipolysis can give more insights into LD dynamics as well.

STED microscopy cannot only result in improvements of the lateral resolution, but with the 3dimensional STED improvements in the z-direction can be achieved as well. In chapter 3 we set-up confocal measurements for 3-dimensional reconstruction of intramyocellular LDs and the LD coat with PLIN5. These 3-dimensional reconstructions can be used to examine how PLIN5 or another protein of interest is surrounding the LD. Most 2-dimensional images acquired on a widefield fluorescence microscope have shown PLIN5 as a rim like structure surrounding the LD [22, 33]. However, when examining 3-dimensional reconstructions of LDs and PLIN5 with CLSM it can be clearly seen that PLIN5 is present on LDs as single or multiple and distinct spots (Chapter 3 ), which is in accordance with 2-dimensional confocal images [34]. These observations indicate that PLIN5 may promote physical contact between LDs and mitochondria [21], thereby directly connecting fuel supply to the furnace.

These super-resolution microscopy techniques show much more details we have never seen before. This improvement in resolution of light microscopy can also be a pitfall. How should we interpret new details we have never seen before? For instance, Große et al. 2016 [35] were able to visualize rings of Bax, a protein involved in autophagy, on a single mitochondria. However, how to determine if the mitochondrial stain is a single mitochondria or a group of clustered mitochondria? In addition, these authors observed that these Bax rings appeared to be speckled. This was never seen before, because the spatial resolution was simply not high enough. These authors discussed that this 
speckled pattern can be due to imperfect antibody decoration or that these rings are composed of different proteins. For optimal resolution also the size of antibodies and labeling density should be taken into account, because the resolution of STED microscopy approaches the size of the complex of primary and secondary antibodies [36]. IgG based antibodies have typically a molecular weight of 150-160 kDa and IgM based antibodies of $900 \mathrm{kDa}$. IgG and IgM have a length of 10-15 nm and 35 $\mathrm{nm}$, respectively [37]. The combination of primary and secondary antibodies can have a length between 30 and $70 \mathrm{~nm}$, approaching the resolution of STED. Thus, rather using the full IgG as an antibody, the application of Fab fragments ( $\sim 50 \mathrm{kDa}$ ) or single domain antibodies ( $15 \mathrm{kDa}$ and also referred to as 'nanobodies') may be favored for super-resolution imaging purposes (Figure 1).

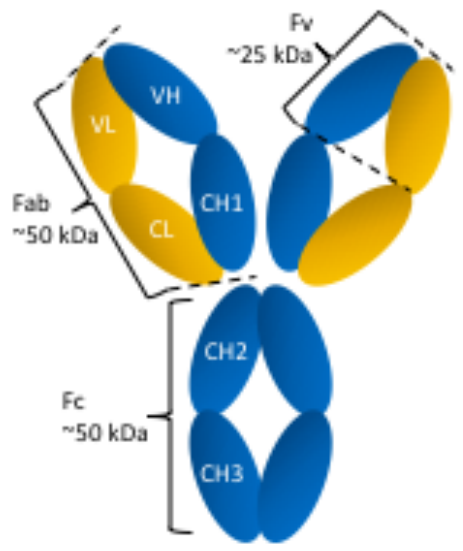

IgG

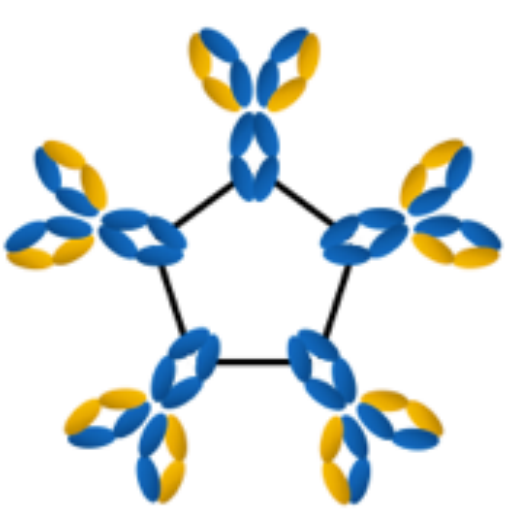

IgM

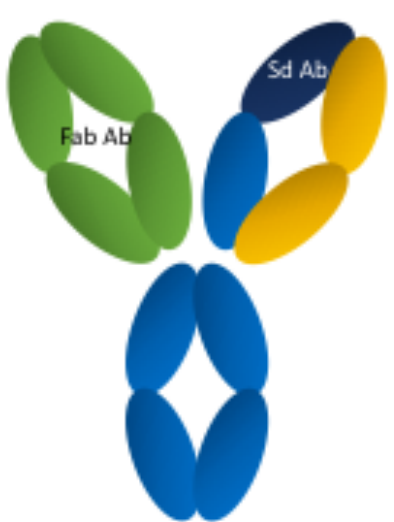

Fab and single domain antibody

Figure 1. Sizes of different types of antibodies that are commonly used in immunofluorescence microscopy (IgG and IgM) and types of antibodies that may be favored for super-resolution fluorescence microscopy (Fragment anti-binding antibody (Fab Ab; depicted in green) and single domain antibody (Sd Ab; depicted in dark blue)). Fab: antigen binding fragment; Fv: variable fragment; Fc: crystallisable fragment.

\section{IN SITU MEASUREMENTS OF LIPID DROPLET COMPOSITION}

The athlete's paradox has been elucidated from several points of views. In addition to LD morphology and location, intramyocellular lipid composition is proposed to clarify this paradox as well. Several studies have studied lipid composition in skeletal muscle by lipid extraction in combination with liquid or gas chromatography with mass spectroscopy to define the specific subspecies [17, 38-43]. Results on skeletal muscle bioactive lipid composition are not conclusive with respect to total diacylglycerol (DAG) and ceramide content, levels of saturation of these intermediates or specific fatty acids esterified in DAGs and ceramides. While mass spectrometry of extracted lipids provides detailed information about fatty acid composition of DAGs and ceramides, 
no distinction can be made between cell membranes and lipid droplets unless membrane fractions are made and lipid droplets are isolated. It has been reported that membrane-bound DAG is associated with insulin resistance, while this is not the case for cytosolic DAG [42, 43]. However, the signal from the cytosolic fraction is not exclusively derived from LDs but may also include other lipids or lipid structures.

In situ techniques, like Coherent Anti-stokes Raman Scattering (CARS), Time-of-Flight Secondary lon Mass Spectroscopy (ToF-SIMS), and Matrix-Assisted Laser Desorption Ionization Mass Spectrometry Imaging (MALDI-MSI), provide spatial information on lipid composition. CARS microscopy gives an insight in average chain length, level of saturation and esterification of fatty acids into triacylglycerol and cholesterol esters [44], while ToF-SIMS is able to provide detailed information about fatty acid composition of lipid species $[45,46]$. Although ToF-SIMS is a useful tool to measure small molecules at organelle level, it lacks sensitivity for examining larger molecules like triacylglycerol. MALDI-MSI has the advantage of detecting these large molecules [47]. In chapter 6 we applied CARS microscopy to examine if lipid characteristics of the myocellular lipid droplets were different between endurance trained athletes and type 2 diabetes patients. Lipid characteristics we assessed were chain length and level of saturation. In addition, lipid characteristics were determined per fiber type by combining CARS microscopy with widefield fluorescence microscopy. Lipid characteristics of the lipid droplets were similar between groups and fiber types with the exception of a few subtle details. Endurance trained athletes had a slightly higher levels of unsaturation and longer chain lengths. If these subtle differences are of relevance for clarifying the athlete's paradox is debatable since results reported so far are inconclusive $[17,40,41]$. In contrast to ToF-SIMS and MALDI-MSI, CARS microscopy is unable to give details on the lipid subtypes. Studies applying ToF-SIMS in rodent skeletal muscle have shown differences in fatty acid compositions of lipid subspecies between obese and non-obese animals [45] or specific high-fat diet-induced alteration in lipid composition of lipid subspecies for saturated and unsaturated fat [46]. Using MALDI-MSI different lipid profiles can be detected in skeletal muscle biopsies of young lean, elderly lean and elderly with metabolic syndrome [48]. At this very moment ToF-SIMS and MALDI-MSI lacking the resolution required for LD specific measurements. In theory ToF-SIMS can achieve a spatial resolution at the $100 \mathrm{~nm}$ level $[30,49]$. However, in practice a resolution of $\sim 1 \mu \mathrm{m}$ is reached in biological samples $[30,49,50]$. This is a consequence of the trade-off that needs to be made between spatial resolution and mass resolving power [49]. The spatial resolution of MALDI-MSI is $25 \mu \mathrm{m}$, although $10 \mu \mathrm{m}$ spatial resolution is reported as well [51]. So, to gain spatial resolution less details on lipid composition can be measured. However, the developments are going fast in this field. Even though the spatial resolution at present is not high enough for applying ToF-SIMS on intramyocellular LDs, MALDI-MSI already 
provides valuable insights into the lipids found in the muscle cells of type 2 diabetes patients and endurance trained athletes (Figure 2). If we successfully can bring down the spatial resolution of ToF-SIMS, we will be capable of measuring individual lipid droplet composition, the effect of coating of LDs with proteins like PLIN5 and associate this with peripheral insulin sensitivity.

(A)

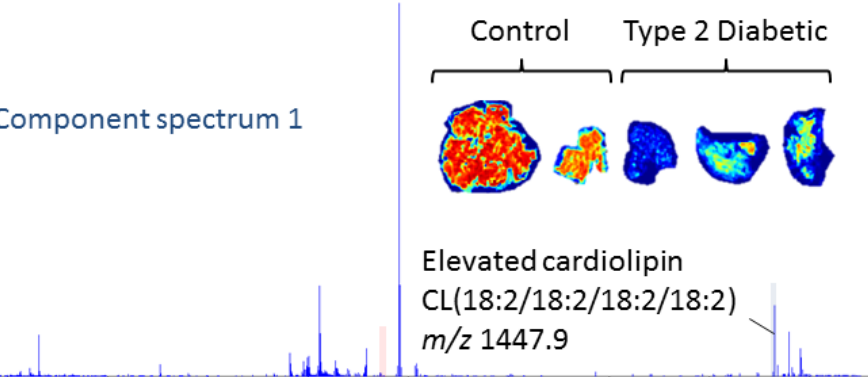

(B)

$$
\text { Component spectrum } 2
$$

Elevated phosphatidylinositols $\mathrm{PI}(36: 2)$ and $(36: 1)$ $\mathrm{m} / \mathrm{z} 861.5$ and 863.5

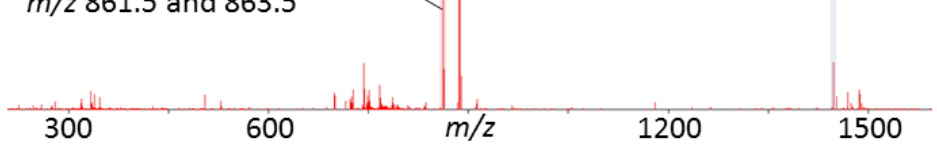

Figure 2. Probabilistic latent semantic analysis (pLSA) reveals biochemical differences in endurance trained athletes (A; Control) and type 2 diabetes patients (B). The insets in A and B show a falsecolor plot demonstrating the relative intensity of the pLSA component spectrum for each pixel. The hotter color, the more closely the spectrum acquired in that pixel resembles the pLSA component spectrum. Preliminary data shows that endurance trained athletes have elevated levels of cardiolipin and type 2 diabetes patients have elevated levels of phosphatidylinositols in skeletal muscle fibers.

\section{FUTURE PERSPECTIVES}

In this thesis human myocellular lipid storage was studied in relation to insulin sensitivity. A microscopy approach was used to study myocellular lipid characteristics. Myocellular lipid storage pattern is different between endurance trained athletes and type 2 diabetes patients. In addition, this pattern is affected upon prolonged fasting. The association between insulin sensitivity and PLIN5 protein content turned out not to be straightforward.

1. What seems to be specific for type 2 diabetes patients is that subsarcolemmal lipid storage in skeletal muscle is high. It can be reasoned that lipid storage in the subsarcolemmal area is non-beneficial, since insulin signaling occurs at the cell membrane. The reason why these lipids are stored beneath the cell membrane in type 2 diabetes remains to be elusive. One of the fundamental questions that needs further elaboration is where new lipid droplets (LDs) are formed in human skeletal muscle. Are these LDs formed in near vicinity of the cell membrane and then transported to the intermyofibrillar compartment or are these LDs formed in both compartments? 
2. In this thesis two-color imaging with STED microscopy was set-up successfully. With the correct settings resolutions of $60 \mathrm{~nm}$ can be reached on a commercial system. With this high resolution more details can be revealed. Interaction between important players in LD dynamics, like ATGL, CGI-58 and PLIN5, can be examined in human skeletal muscle. By using prolonged fasting, acute exercise or lipid infusion LDs in human skeletal can be modulated and insights into the LD dynamics can be achieved. However, for measuring real LD dynamics in skeletal muscle primary myotubes needs to be used. These myotubes can be challenged with a lipid overload or stimulated to release fatty acids whereby LDs and proteins of interest are followed over time with live super-resolution microscopy. However, steps need to be made to be able to separate multiple colors.

3. PLIN5 protein content is higher in endurance trained athletes compared to lean and obese sedentary subjects, and type 2 diabetes patients. However, the association between PLIN5 protein content and insulin sensitivity is not straightforward. We observed that PLIN5 only predicted insulin sensitivity when maximal oxidative capacity is taken into account. So, what the exact relationship is between PLIN5, oxidative capacity and insulin sensitivity is something that needs to be explored in more depth.

4. Results on lipid composition of skeletal muscle are inconclusive. In situ techniques like CARS, MALDI-MSI, and ToF-SIMS are developing quickly. MALDI-MSI and ToF-SIMS are of interest since fatty acid composition of lipid subtypes can be measured in a location specific manner. This will be of more interest when developments result in higher resolutions to distinguish cell membranes and individual lipid droplets. At the moment few studies have examined membrane fractions and cytosolic fractions with mass spectroscopy. However, cytosolic fractions do not only contain LDs, but also other cellular components. ToF-SIMS would be a nice application to measure LD fatty acid content and to examine if subsarcolemmal and intermyofibrillar LDs differ in composition.

\section{REFERENCES}

1. Goodpaster, B.H., et al., Skeletal muscle lipid content and insulin resistance: evidence for a paradox in endurance-trained athletes. J Clin Endocrinol Metab, 2001. 86(12): p. 5755-61.

2. Goodpaster, B.H., et al., Intramuscular lipid content is increased in obesity and decreased by weight loss. Metabolism, 2000. 49(4): p. 467-72.

3. Pan, D.A., et al., Skeletal muscle triglyceride levels are inversely related to insulin action. Diabetes, 1997. 46(6): p. 983-8.

4. Sinha, S., et al., Proton magnetic resonance spectroscopy and biochemical investigation of type 2 diabetes mellitus in Asian Indians: observation of high muscle lipids and C-reactive protein levels. Magn Reson Imaging, 2009. 27(1): p. 94-100. 
5. Sinha, S., et al., Subclinical inflammation and soleus muscle intramyocellular lipids in healthy Asian Indian males. Clin Endocrinol (Oxf), 2005. 63(3): p. 350-5.

6. Krssak, M., et al., Intramyocellular lipid concentrations are correlated with insulin sensitivity in humans: a $1 H$ NMR spectroscopy study. Diabetologia, 1999. 42(1): p. 113-6.

7. He, J., B.H. Goodpaster, and D.E. Kelley, Effects of weight loss and physical activity on muscle lipid content and droplet size. Obes Res, 2004. 12(5): p. 761-9.

8. Nielsen, J., et al., Increased subsarcolemmal lipids in type 2 diabetes: effect of training on localization of lipids, mitochondria, and glycogen in sedentary human skeletal muscle. Am J Physiol Endocrinol Metab, 2010. 298(3): p. E706-13.

9. Samjoo, I.A., et al., Markers of skeletal muscle mitochondrial function and lipid accumulation are moderately associated with the homeostasis model assessment index of insulin resistance in obese men. PLoS One, 2013. 8(6): p. e66322.

10. Li, Y., et al., Subsarcolemmal lipid droplet responses to a combined endurance and strength exercise intervention. Physiol Rep, 2014. 2(11).

11. Devries, M.C., et al., Endurance training modulates intramyocellular lipid compartmentalization and morphology in skeletal muscle of lean and obese women. J Clin Endocrinol Metab, 2013. 98(12): p. 4852-62.

12. Herms, A., et al., AMPK activation promotes lipid droplet dispersion on detyrosinated microtubules to increase mitochondrial fatty acid oxidation. Nat Commun, 2015. 6: p. 7176.

13. Loussert Fonta, C. and B.M. Humbel, Correlative microscopy. Arch Biochem Biophys, 2015. 581: p. 98110.

14. de Boer, P., J.P. Hoogenboom, and B.N. Giepmans, Correlated light and electron microscopy: ultrastructure lights up! Nat Methods, 2015. 12(6): p. 503-13.

15. Watanabe, S., et al., Protein localization in electron micrographs using fluorescence nanoscopy. Nat Methods, 2011. 8(1): p. 80-4.

16. Sanders, M.A., et al., Endogenous and Synthetic ABHD5 Ligands Regulate ABHD5-Perilipin Interactions and Lipolysis in Fat and Muscle. Cell Metab, 2015. 22(5): p. 851-60.

17. Amati, F., et al., Skeletal muscle triglycerides, diacylglycerols, and ceramides in insulin resistance: another paradox in endurance-trained athletes? Diabetes, 2011. 60(10): p. 2588-97.

18. Koves, T.R., et al., PPARgamma coactivator-1alpha contributes to exercise-induced regulation of intramuscular lipid droplet programming in mice and humans. J Lipid Res, 2013. 54(2): p. 522-34.

19. Louche, K., et al., Endurance exercise training up-regulates lipolytic proteins and reduces triglyceride content in skeletal muscle of obese subjects. J Clin Endocrinol Metab, 2013. 98(12): p. 4863-71.

20. Bakke, S.S., et al., Myotubes from severely obese type 2 diabetic subjects accumulate less lipids and show higher lipolytic rate than myotubes from severely obese non-diabetic subjects. PLoS One, 2015. 10(3): p. e0119556.

21. Wang, H., et al., Perilipin 5, a lipid droplet-associated protein, provides physical and metabolic linkage to mitochondria. J Lipid Res, 2011. 52(12): p. 2159-68.

22. Bosma, M., et al., The lipid droplet coat protein perilipin 5 also localizes to muscle mitochondria. Histochem Cell Biol, 2012. 137(2): p. 205-16.

23. Ramos, S.V., et al., Higher PLIN5 but not PLIN3 content in isolated skeletal muscle mitochondria following acute in vivo contraction in rat hindlimb. Physiol Rep, 2014. 2(10).

24. Ramos, S.V., et al., Changes in mitochondrial PLIN3 and PLIN5 protein content in rat skeletal muscle following endurance training and acute stimulated contraction. Exp Physiol, 2015.

25. Bartholomew, S.R., et al., Distinct cellular pools of perilipin 5 point to roles in lipid trafficking. Biochim Biophys Acta, 2012. 1821(2): p. 268-78.

26. Wolins, N.E., D.L. Brasaemle, and P.E. Bickel, A proposed model of fat packaging by exchangeable lipid droplet proteins. FEBS Lett, 2006. 580(23): p. 5484-91. 
27. MacPherson, R.E., et al., Skeletal muscle PLIN proteins, ATGL and CGI-58, interactions at rest and following stimulated contraction. Am J Physiol Regul Integr Comp Physiol, 2013. 304(8): p. R644-50.

28. Macpherson, R.E., et al., Skeletal muscle PLIN3 and PLIN5 are serine phosphorylated at rest and following lipolysis during adrenergic or contractile stimulation. Physiol Rep, 2013. 1(4): p. e00084.

29. Mason, R.R., et al., Cellular localization and associations of the major lipolytic proteins in human skeletal muscle at rest and during exercise. PLoS One, 2014. 9(7): p. e103062.

30. Daemen, S., et al., Microscopy tools for the investigation of intracellular lipid storage and dynamics. Mol Metab, 2016. 5(3): p. 153-63.

31. Hell, S.W. and J. Wichmann, Breaking the diffraction resolution limit by stimulated emission: stimulated-emission-depletion fluorescence microscopy. Opt Lett, 1994. 19(11): p. 780-2.

32. Schermelleh, L., R. Heintzmann, and H. Leonhardt, A guide to super-resolution fluorescence microscopy. J Cell Biol, 2010. 190(2): p. 165-75.

33. Harris, L.A., et al., Perilipin 5 - driven lipid droplet accumulation in skeletal muscle stimulates the expression of fibroblast growth factor 21. Diabetes, 2015.

34. Shepherd, S.O., et al., Sprint interval and traditional endurance training increase net intramuscular triglyceride breakdown and expression of perilipin 2 and 5. J Physiol, 2013. 591(3): p. 19.

35. Große, L., et al., Bax assembles into large ring-like structures remodeling the mitochondrial outer membrane in apoptosis. EMBO J, 2016. 35(4): p. 402-13.

36. Lau, L., et al., STED microscopy with optimized labeling density reveals 9-fold arrangement of a centriole protein. Biophys J, 2012. 102(12): p. 2926-35.

37. Saber, R., et al., High resolution imaging of IgG and IgM molecules by scanning tunneling microscopy in air condition. Scientia Iranica, 2011. 18(6): p. 1643-1646.

38. Bergman, B.C., et al., Increased intramuscular lipid synthesis and low saturation relate to insulin sensitivity in endurance-trained athletes. J Appl Physiol, 2010. 108(5): p. 1134-41.

39. Moro, C., et al., Influence of gender, obesity, and muscle lipase activity on intramyocellular lipids in sedentary individuals. J Clin Endocrinol Metab, 2009. 94(9): p. 3440-7.

40. Dube, J.J., et al., Exercise-induced alterations in intramyocellular lipids and insulin resistance: the athlete's paradox revisited. Am J Physiol Endocrinol Metab, 2008. 294(5): p. E882-8.

41. Dube, J.J., et al., Effects of weight loss and exercise on insulin resistance, and intramyocellular triacylglycerol, diacylglycerol and ceramide. Diabetologia, 2011. 54(5): p. 1147-56.

42. Bergman, B.C., et al., Localisation and composition of skeletal muscle diacylglycerol predicts insulin resistance in humans. Diabetologia, 2012. 55(4): p. 1140-50.

43. Nowotny, B., et al., Mechanisms underlying the onset of oral lipid-induced skeletal muscle insulin resistance in humans. Diabetes, 2013. 62(7): p. 2240-8.

44. Billecke, N., et al., Perilipin 5 mediated lipid droplet remodelling revealed by coherent Raman imaging. Integr Biol (Camb), 2015.

45. Magnusson, Y.K., et al., TOF-SIMS analysis of lipid accumulation in the skeletal muscle of ob/ob mice. Obesity (Silver Spring), 2008. 16(12): p. 2745-53.

46. Sjovall, P., et al., Dietary uptake of omega-3 fatty acids in mouse tissue studied by time-of-flight secondary ion mass spectrometry (TOF-SIMS). Anal Bioanal Chem, 2015. 407(17): p. 5101-11.

47. Goto-Inoue, N., et al., Visualization of dynamic change in contraction-induced lipid composition in mouse skeletal muscle by matrix-assisted laser desorption/ionization imaging mass spectrometry. Anal Bioanal Chem, 2012. 403(7): p. 1863-71.

48. Gueugneau, M., et al., Skeletal muscle lipid content and oxidative activity in relation to muscle fiber type in aging and metabolic syndrome. J Gerontol A Biol Sci Med Sci, 2015. 70(5): p. 566-76.

49. Boxer, S.G., M.L. Kraft, and P.K. Weber, Advances in imaging secondary ion mass spectrometry for biological samples. Annu Rev Biophys, 2009. 38: p. 53-74.

50. Billecke, N., et al., Chemical imaging of lipid droplets in muscle tissues using hyperspectral coherent Raman microscopy. Histochem Cell Biol, 2014. 141(3): p. 263-73. 


\section{CHAPTER 8}

51. McDonnell, L.A. and R.M. Heeren, Imaging mass spectrometry. Mass Spectrom Rev, 2007. 26(4): p. 606-43. 


\section{Appendices}

Summary

Samenvatting

Valorization

Risk analysis and technology assessment

Dankwoord

List of publications

Curriculum Vitae 


\section{SUMMARY}

The prevalence of type 2 diabetes (T2DM) is increasing worldwide. Lipid content in skeletal muscle fibers is higher in T2DM than in control subjects. Intramyocellular lipid (IMCL) content is negatively associated with insulin sensitivity. However, endurance trained athletes have IMCL levels similar to patients with T2DM, whilst being very insulin sensitive, the so-called athlete's paradox. Therefore, it has been suggested that lipid intermediates, like diacylglycerol and ceramides, interfere with insulin sensitivity and that total IMCL content is just a surrogate marker of these bioactive lipid intermediates. However, data in humans is on the involvement of bioactive lipids in impeding insulin sensitivity remains contradictory.

IMCL is dispersed in small lipid droplets (LDs). LDs have different characteristics, like size, number, location, lipid composition, and protein coat with a variety of proteins. The content of LD coating proteins and lipid composition is most commonly measured in muscle homogenates, and thus information about location is lacking. Microscopy is a tool frequently used to examine LD size, number and location. In this thesis LD characteristics in (mainly) skeletal muscle in relation to insulin sensitivity and the role Perilipin 5 (PLIN5) herein have been examined by fluorescent confocal microscopy.

In chapter 2 the effect of a 5-day high-fat high-calorie (HFHC) diet on IMCL content and proteins involved in LD dynamics in young, lean Caucasians and South Asians was examined. While Caucasians remain insulin sensitive after this dietary intervention, South Asians did develop insulin resistance. In contrast to our hypothesis that LD dynamics will be different between these two groups, the diet-induced changes in IMCL and PLIN5 protein content were comparable in Caucasians and South Asians. However, South Asians had higher levels of PLIN5 protein before and after the diet. Other proteins involved in LD dynamics did not change and were not differentially affected upon the HFHC diet. Remarkably, Caucasians who increased the most in PLIN5 protein content dropped the least in insulin sensitivity. On the contrary, South Asians possessed higher levels of lipid peroxidation whilst having higher levels of PLIN5. PLIN5 seems to play a protective role against the development of insulin resistance upon a 5-day HFHC diet in Caucasians, which is (for some reason to be investigated) not the case in South Asians.

While in chapter 2 content of proteins involved in LD dynamics and IMCL were measured in muscle homogenates, little is known what happens to the LD morphology and location of these proteins. For example, PLIN5 is not only present on the LD surface, but is also observed in cytosolic fractions. The subcellular distribution of these proteins might be of importance for the understanding of LD 
dynamics. In chapter 3 a confocal microscopy approach to study LD morphology and coating of the LD in human skeletal muscle was successfully set-up. Furthermore, 3D reconstructions of LDs and PLIN5 were made which revealed that PLIN5 is not present in a rim-like structure encompassing LD, but rather presents at distinct punctate loci on the LD. In addition, electron microscopy images have shown that PLIN5 is also present in mitochondria. This observation is not confirmed in literature, thus there still is an ongoing debate as to whether PLIN5 indeed is present in the mitochondria. To confirm our previous findings in electron microscopy we set-up a STED-based approach to image PLIN5 and examine if PLIN5 is indeed present at the inner or outer mitochondrial membrane as was suggested by our previous electron microscopy observations. Although we successfully distinguished the inner and outer mitochondrial membrane by using STED microscopy, we did not detect PLIN5 at the inner and outer mitochondrial membrane, but rather in close proximity of mitochondria. Concluded from this chapter is that confocal microscopy is sufficient to examine LD morphology and to some extend LD protein decoration. However, for a more detailed analysis of LD decoration and LD-mitochondria interaction super-resolution techniques, like STED microscopy, is warranted.

The developed confocal microscopy approach to study LD morphology and protein decoration in relation to insulin sensitivity was applied in chapter 4. Healthy, young, lean males were fasted for 60 hours. During this 60-hour fast, subjects developed insulin resistance and increased IMCL content. Remarkably, the subjects who increased most in IMCL became the least insulin resistant. IMCL content was analyzed in more detail with confocal microscopy and was elevated due to larger and more LDs upon fasting. Although total PLIN5 protein content was not affected by prolonged fasting, the fraction of PLIN5 was associated with LDs after fasting had increased, suggesting a translocation of PLIN5 from the cytosolic pool to the LD. Upon making the distinction between LDs with and devoid of PLIN5, we were capable to see that PLIN5 coated LDs increased in size and number upon fasting. The increase in PLIN5 coated LDs was positively associated with the reduction in insulin sensitivity. Thus, the subjects who were able to create more PLIN5 coated LDs were less vulnerable for the development of fasting-induced insulin resistance.

In a situation of physiological insulin resistance (prolonged fasting) it seems like that PLIN5 promotes benign lipid storage. In chapter 5 it was hypothesized that with similar levels of IMCL, the number of PLIN5 coated LDs is a determinant of insulin sensitivity. In this study, young endurance trained athletes, young lean sedentary subjects, obese sedentary subjects, and T2DM patients were included. Athletes and T2DM patients were matched for total IMCL content. It was observed that athletes and T2DM patients had a different lipid storage pattern; athletes had more LDs, while 
T2DM patients had larger LDs. Despite different levels of PLIN5 protein content (higher in athletes), the lipid storage pattern of IMCL with and devoid of PLIN5 showed a similar pattern as the total IMCL content. None of the LD parameters and PLIN5 protein content did correlate with insulin sensitivity. Maximal oxidative capacity was the main predictor for insulin sensitivity. PLIN5 protein content and number of PLIN5 coated LDs contributed significantly to the predicted insulin sensitivity, but only when maximal oxidative capacity was taken into account. Thus, PLIN5 in itself cannot explain in a direct way the athlete's paradox and may need a concomitant high maximal oxidative capacity.

In chapter 6 the lipid storage pattern was examined in a fiber type specific manner in the same group of subjects as selected in chapter 5. Besides LD morphology, the composition of the LD with respect to fatty acid composition might be an important contributor to the development of insulin resistance. CARS microscopy was applied to measure the chemical composition of LDs in skeletal muscle sections of endurance trained athletes and T2DM patients in a fiber type specific and label free fashion. In contrary to what was anticipated, the chemical composition of LDs found in skeletal muscle samples of athletes was strikingly similar to the composition found in T2DM patients. LDs in type II fibers contained significantly more saturated fat in athletes than in patients with T2DM. Next to LD composition, LD morphology and location was assessed in a fiber type specific way. Excess IMCL stored in type I fibers of athletes was due to more LDs, while T2DM stored surplus IMCL in type II fibers due to larger LDs and more storage in the subsarcolemmal (SS) area. This increased IMCL content in the SS area was due to more and larger LDs. LD number in type I fibers was positively associated with insulin sensitivity and LD size in type II fibers was negatively associated with insulin sensitivity. From this study, it was concluded that lipid storage pattern and location is an important player in the athlete's paradox.

The confocal fluorescence measurements of LD morphology and location can also be applied in other organs. In chapter $\mathbf{7}$ confocal fluorescence imaging of LDs was applied in left ventricle biopsies from patients with and without T2DM who underwent surgery for coronary artery disease (CAD). As a control group, patients without T2DM who needed an aortic valve replacement (considered to be metabolically normal) were included. Cardiac lipid content, LD size and number, and LD location were examined in these 3 groups. LD location was examined relative to the contractile elements and the T-tubular system. No significant differences in lipid content, LD size, and LD number was observed among groups. However, lipid content increased stepwise from non-CAD to CAD with T2DM. This stepwise increase of lipid content was due to a higher number of LDs. LD location 
relative the contractile elements appeared not to be different However, it seems like that both CAD groups had more LDs stored in close vicinity of the T-tubuli than the non-CAD group. LD morphology was not associated with whole body insulin sensitivity. Whether these LD parameters are related to cardiac dysfunction needs further examination.

In conclusion, confocal fluorescence microscopy is a valuable tool to investigate IMCL in more detail and provides insights in location, and LD decoration. Studies performed in this thesis show that LD morphology is different in situations of insulin resistance compared to insulin sensitive situations. Not only LD morphology is of importance, but the proteins coating the LDs are as well. Confocal microscopy revealed that PLIN5 protein content and PLIN5 coated LDs are involved in benign IMCL storage and thereby may contribute to the maintenance of insulin sensitivity under otherwise insulin desensitizing conditions. However, the link between PLIN5 and insulin sensitivity did not appear to be direct and also other factors, like maximal oxidative capacity, and interaction of PLIN5 with lipases and other LD coating proteins, appear to affect the protective nature of PLIN5 in benign IMCL storage. 


\section{SAMENVATTING}

De prevalentie van type 2 diabetes (T2DM) neemt wereldwijd toe. In patiënten met T2DM wordt meer vet opgeslagen in de skeletspiervezels dan in de spiervezels van gezonde personen. De hoeveelheid intramyocellulaire lipiden (IMCL) zijn negatief geassocieerd met insulinegevoeligheid. Echter, duurgetrainde atleten hebben hoeveelheden IMCL die vergelijkbaar zijn met die van patiënten met T2DM terwijl ze insulinegevoelig zijn, de zogenaamde atleten paradox. Zodoende werd er gesuggereerd dat lipide intermediairen, zoals diacylglycerol en ceramide, interfereren met insulinegevoeligheid en dat de hoeveelheid IMCL niet meer is dan een marker voor de hoeveelheid van deze intermediairen. De resultaten met betrekking tot de aanwezigheid van deze lipide intermediaieren bij patienten met T2DM zijn dan ook tegenstrijdig.

IMCL wordt opgeslagen in kleine lipide druppels (LDs). Deze LDs hebben verschillende karakteristieken, zoals grootte, aantal, locatie, lipide compositie en de eiwitten die zich op deze druppels bevinden. Het soort en de hoeveelheid van de eiwitten die de LD decoreren en de samenstelling van het vet in de LD wordt vaak gemeten in spierhomogenaten. Door het meten in spierhomogenaten gaat kennis met betrekking tot de locatie en de soort van deze eiwitten verloren. Microscopie wordt vaak gebruikt voor het bepalen van de LD grootte, aantal en locatie. In dit proefschrift is de relatie tussen de karakteristieken van LDs in (voornamelijk) skeletspier met insulinegevoeligheid en de rol van Perilipine 5 (PLIN5), één van de LD decorerende eiwitten, onderzocht met behulp van fluorescentie confocaal microscopie.

In hoofdstuk $\mathbf{2}$ is het effect van een 5-daags hoog vet, hoog calorie (HVHC) dieet op de hoeveelheid IMCL en eiwitten betrokken bij de LD dynamiek in jonge, slanke Europeanen en Zuid Aziaten onderzocht. Terwijl Europeanen insulinegevoelig bleven op dit 5-daags HVHC dieet, ontwikkelden de Zuid Aziaten insuline resistentie. Verwacht werd dat de LD dynamiek in deze twee groepen van elkaar zou verschillen. Het HVHC dieet veroorzaakte echter vergelijkbare veranderingen in hoeveelheid IMCL en PLIN5 in beide groepen. Wel hadden Zuid Aziaten voor en na het dieet meer PLIN5 eiwit. De hoeveelheid van andere eiwitten betrokken bij de LD dynamiek veranderde niet ten gevolge van het dieet of waren verschillend tussen de groepen. Opmerkelijk was dat de Europeanen die de grootste toename hadden van PLIN5 het minste daling in insulinegevoeligheid hadden, dit zou kunnen suggereren dat PLIN5 betrokken is bij de handhaving van insuline gevoeligheid zelfs na consumptie van een HVHC dieet. In tegenstelling tot Europeanen vertoonden de Zuid Aziaten ondanks hogere PLIN5 concentratie meer insuline resistentie en meer lipide peroxidatie na een 5daags HVHC dieet. De onderliggende oorzaak voor dit verschil in respons tussen beide etniciteiten is nog onbekend. 
In hoofdstuk 2 zijn de hoeveelheden van eiwitten betrokken bij LD dynamiek en IMCL gemeten in spierhomogenaten. Hierdoor is de morfologie van de LD onbekend en is tevens onduidelijk hoe de betrokken eiwitten al dan niet op de LD aanwezig zijn. Deze locatie van eiwitten betrokken bij de LD dynamiek kan van belang zijn voor het begrijpen van deze dynamiek. In hoofdstuk $\mathbf{3}$ is met succes een confocale microscopische benadering opgezet om LD morfologie en de eiwitdecoratie in humane skeletspier te volgen en kwantificeren. Verder zijn er 3D reconstructies van LDs en PLIN5 gemaakt. Deze reconstructies laten zien dat PLIN5 niet de gehele LD omringd, maar aanwezig is specifieke locaties op de LD. Daarnaast laten elektronen microscopie beelden zien dat PLIN5 ook aanwezig is in mitochondria. Dit wordt echter door bevindingen van anderen in de literatuur niet bevestigd. Om onze eerdere bevindingen met elektronen microscopie te bevestigen hebben we dit benaderd met STED microscopie en of PLIN5 dan op het binnen- of buitenmembraan aanwezig is. STED microscopie heeft voldoende spatiele resolutie om het binnen- en buitenmembraan van elkaar te onderscheiden. In tegenstelling tot de beelden verkregen met de elektronen microscopie, geven de STED beelden geen aanleiding te denken dat PLIN5 aanwezig is op de binnen- en buitenmembraan van het mitochondrion, maar laat STED wel zien dat PLIN5 in de directe nabijheid van de mitochondria gevonden wordt op plekken waar het de interactie tussen de mitochondria en de LD lijkt te kunnen bevorderen.

Geconcludeerd kan worden dat met confocaal microscopie LD morfologie bestudeerd kan worden en dat voor een gedetailleerde analyse van de LD decoratie en LD-mitochondria interactie superresolutie technieken, zoals STED, de aangewezen methoden lijken.

De methode om LD morfologie en eiwitdecoratie te meten in relatie met insulinegevoeligheid is toegepast in hoofdstuk 4. Jonge, gezonde, slanke mannen werden gevast voor 60 uur. Tijdens deze langdurige perioden van vasten ontwikkelden de proefpersonen insuline resistentie en vertoonden ze een toename in de hoeveelheid IMCL. Opvallend was dat de proefpersonen die de grootste toename hadden in IMCL de kleinste daling in insulinegevoeligheid lieten zien. IMCL opslag werd in detail geanalyseerd met confocaal microscopie en de toename in IMCL werd veroorzaakt door zowel grotere als meer LDs. Hoewel de hoeveelheid PLIN5 niet toenam door langdurig vasten nam de fractie PLIN5 die geassocieerd werd met LDs toe. Dit suggereert dat er een PLIN5 translocatie plaatsvond van de cytosolaire pool naar de LDs. Wanneer de LDs werden onderverdeeld in LDs gedecoreerd met en zonder PLIN5 werd duidelijk dat de toename in LD grootte en aantal kwam door een toename in grootte en aantal van de PLIN5 gedecoreerde LDs. De toename in aantal PLIN5 gedecoreerde LDs was positief geassocieerd met de afname in insuline gevoeligheid. Dus de 
proefpersonen die in staat waren om meer PLIN5 gedecoreerde LDs te creëren waren tot op een bepaalde hoogte beschermd tegen de ontwikkeling van vasten geïnduceerde insuline resistentie.

In situaties van fysiologische insuline resistentie (langdurig vasten) lijkt het erop dat PLIN5 de opslag van vet in IMCL opslag bevordert op een wijze die de insuline gevoeligheid niet negatief lijkt te beïnvloeden. In hoofdstuk 5 werd de hypothese getest of in het geval van vergelijkbare hoeveelheden IMCL, de fractie LD die met PLIN5 zijn gedecoreerd van belang zijn voor de handhaving van insuline gevoeligheid. In deze studie werden jonge duurgetrainde atleten, jonge slanke sedentaire proefpersonen, obese sedentaire proefpersonen en T2DM patiënten geïncludeerd. Atleten en T2DM patiënten waren gematched voor hun hoeveelheid IMCL. Ondanks vergelijkbare hoeveelheid IMCL, was het opslagpatroon van vet in de LD duidelijk verschillend tussen atleten en T2DM patiënten; atleten hadden meer LDs en T2DM patiënten grotere LDs. Ondanks de observatie dat atleten meer PLIN5 hadden dan de T2DM patiënten, was het IMCL opslagpatroon voor LDs met en zonder PLIN5 gelijk aan het patroon van de totale IMCL pool. Noch LD aantal noch LD grootte correleerde met insulinegevoeligheid. De belangrijkste determinant van insuline gevoeligheid was in deze populaties de maximale oxidatieve capaciteit. De hoeveelheid PLIN5 eiwit en het aantal PLIN5 gedecoreerde LDs zijn voorspellende parameters voor de mate van insulinegevoeligheid wanneer maximale oxidatieve capaciteit meegenomen wordt in het model. Het lijkt zodoende niet waarschijnlijk dat PLIN5 op een directe wijze de atleten paradox verklaart. De analyses laten echter wel zien dat PLIN5 van voorspellende waarde is indien er rekening wordt gehouden met de maximale oxidatieve capaciteit.

In hoofdstuk 6 is onderzocht of IMCL in de spier wordt opgeslagen op een spiervezeltype specifieke wijze. Dit was gemeten in dezelfde groepen zoals in hoofdstuk 5. Naast LD morfologie, kan de vetsamenstelling in de LD een belangrijke bijdrage leveren aan de ontwikkeling van insuline resistentie. Middels het gebruik van CARS microscopie kan zonder het verdere gebruik van labels een beeld worden verkregen van de chemische samenstelling van LDs. Dit type microscopie is toegepast in dunne coupes van spierbiopten van duurgetrainde atleten en T2DM patiënten. De chemische samenstelling van de LDs in de spier van atleten vertoonde verbazend veel gelijkenis met de chemische samenstelling van de LDs in de spier van patiënten met T2DM. LDs in type II vezels van atleten bevatten iets meer gesatureerde vetten dan de lipide druppels in de type II vezels van patiënten met type2 diabetes. Naast de LD samenstelling zijn ook LD morfologie en locatie per vezeltype gemeten. Hieruit blijkt dat atleten IMCL hoofdzakelijk opslaan in type I vezels in de vorm van meer LDs, terwijl de IMCL opslag van T2DM patiënten in type II vezels gekarakteriseerd wordt 
door opslag in grote LDs. Daarnaast is de opslag van IMCL in het subsarcolemmale (SS) gebied in patienten met T2DM meer uitgesproken dan in atleten, dit komt zowel door het hebben van meer LDs als het hebben van grotere LDs bij de patiënten met T2DM. Het aantal LDs in type I vezels correleert positief en de LD grootte in type II vezels correleert negatief met insulinegevoeligheid. Vanuit deze studie werd geconcludeerd dat het IMCL opslagpatroon en de locatie bijdraagt aan het bestaan van de atleten paradox.

De LD morfologie en locatie metingen door middel van confocaal microscopie kan ook toegepast worden in andere organen. In hoofdstuk $\mathbf{7}$ is fluorescentie confocaal microscopie toepast om beelden te maken van LDs in biopten van het linker ventrikel van patiënten met en zonder T2DM die geopereerd moesten worden aan vernauwingen van de kransslagaders (CAD). Patiënten zonder T2DM die geopereerd moesten worden om de aortaklep te vervangen (een groep die niet -of in mindere mate- wordt gekarakteriseerd door metabole verstoringen) werd geïncludeerd als controlegroep. De hoeveelheid cardiale lipiden, LD grootte, LD aantal en LD locatie werden in deze 3 groepen gekwantificeerd. LD locatie werd bepaald ten op zichtte van de contractiele elementen en het T-tubulaire systeem. Er waren geen significante verschillen in hoeveelheid lipiden, LD grootte en LD aantal tussen de groepen. Echter was er een stapsgewijze toename van cardiale lipiden van nonCAD tot CAD met T2DM. Deze stapsgewijze toenamen was een gevolg van meer LDs. LD locatie ten op zichtte van de contractiele elementen was niet verschillend tussen de groepen, maar de CADgroepen neigde naar de opslag van meer LDs in de directe nabijheid van de T-tubuli in vergelijking met de non-CAD groep. LD morfologie associeerde niet met insuline gevoeligheid. Of de gemeten LD parameters gerelateerd zijn aan het minder functioneren van het hart vereist verder onderzoek.

Geconcludeerd kan worden dat confocaal fluorescentie microscopie een waardevol instrument is om IMCL in meer detail te onderzoeken en om inzicht te geven in LD locatie en decoratie. Onderzoeken uitgevoerd in dit proefschrift tonen aan dat LD morfologie in situaties van insuline resistentie verschilt van situaties van insuline gevoeligheid. LD morfologie is niet de enige belangrijke factor, maar ook eiwitten die de LDs decoreren spelen hierin een rol. Met confocaal microscopie hebben we laten zien dat de hoeveelheid PLIN5 en PLIN5 gedecoreerde LDs betrokken zijn bij de veilige opslag van IMCL zodat insulinegevoeligheid kan worden behouden. In dit proefschrift beschrijven we echter ook situaties waarin PLIN5 niet altijd direct gerelateerd is aan insulinegevoeligheid. Dit wijst erop dat andere factoren, zoals maximale oxidatieve capaciteit en interactie tussen PLIN5 met lipasen en andere LD decoratie-eiwitten, wellicht nodig zijn om IMCL opslag te bevorderen op een wijze die niet interfereert met insuline gevoeligheid. 


\section{VALORIZATION}

\section{Social relevance}

The obesity epidemic is growing worldwide and has doubled since 1980 according to the World Health Organization (WHO). In 2014 39\% of the adults were overweight and 13\% were obese. The rise in obesity is not only a problem of well-developed countries, but is on the rise in developing countries as well. In addition, worldwide more people are obese than people who are underweight. Obesity is a major risk factor for cardiovascular diseases and type 2 diabetes. The prevalence of diabetes increased from $4.7 \%$ in 1980 to $8.5 \%$ in 2014 and when looking to absolute numbers the amount of people with diabetes has a four-fold increase in this period. According to the International Diabetes Federation 90\% from all people who have diabetes has type 2 diabetes (T2DM). Due to increasing rates of obesity and T2DM health care costs are on the rise as well. Not only diabetes itself leads to increased cost, but comorbidities contribute as well. Consequences of uncontrolled diabetes that can occur are blindness, cardiovascular diseases, kidney failure and lower extremity amputations.

Early treatment of T2DM and recognition of symptoms at an early stage will prevent or delay the development of comorbidities. In addition, quality of life will improve or be maintained better if blood glucose can be well-controlled. Much can be gained on the early recognition of symptoms of T2DM. According to the WHO $24 \%$ to $62 \%$ of the people with diabetes is undiagnosed and thus untreated. These people are at a higher risk for developing comorbidities, which will burden the health care costs even more. Nowadays treatment of T2DM consists of glucose lowering medication, diet, increasing physical activity or a combination of these. However, T2DM is a complex metabolic disease whereby underlying mechanisms are yet not completely understood. Understanding these underlying mechanisms might result in new treatment strategies.

\section{Scientific background}

Development of obesity and T2DM is a consequence of a sustained positive energy balance. This positive energy balance can result from a high calorie intake or a reduction in physical activity or both. Obesity and T2DM coincides with lipid storage, not only in adipose tissue, but in non-adipose tissues like the liver, heart and skeletal muscle as well. This pattern of ectopic lipid storage in skeletal muscle associates with insulin resistance. Skeletal muscle is an important organ for whole body glucose homeostasis, as it takes up $80 \%$ of the glucose upon meal consumption. However, lipid storage in skeletal muscle is not consistently associated with insulin resistance. In contrast to T2DM patients, endurance-trained athletes store similar amounts of lipids in their skeletal muscle as 
patients with T2DM whilst very insulin sensitive. These ectopic lipids are stored in lipid droplets which are nowadays recognized as dynamic organelles. Athletes store lipids in their skeletal muscle as a readily available energy source for performance. Release of lipids for oxidation and rates of fat oxidation are matched. In contrast, T2DM patients store lipids in skeletal muscle as a consequence of a misbalance between fat oxidation rate and fatty acid supply to the mitochondria. This misbalance can lead to lipotoxic events resulting in insulin resistance of the skeletal muscle. Understanding the role of the dynamics of lipid droplets in skeletal muscle, i.e. the release and storage of fatty acids, may give insights in the relationship between ectopic lipid storage and insulin resistance. This thesis aimed to study the characteristics of skeletal muscle lipid droplets in relation to insulin sensitivity to gain a better understanding of the negative association of lipid storage in the skeletal muscle with insulin resistance. These lipid droplet characteristics (lipid droplet size, number, location and chemical composition) were studied in this thesis in human subjects possessing a wide phenotypical range as well as in subjects challenged with a high-fat diet or during a prolonged fast.

\section{Target groups}

In 2010 the prevalence of physical inactivity in adults was 23\% according to the WHO. Physical activity is the best strategy to combat obesity and T2DM. In addition, physical inactivity contributes to the development of obesity and T2DM. Changing from a sedentary lifestyle, while being obese, is not easy, especially when it is not under supervision. So, other ways of treating obesity and T2DM are warranted. Understanding the role of muscular fat in the development of skeletal muscle insulin resistance may lead to new target treatments.

This thesis focused on lipid droplet characteristics in human subjects and revealed conditions under which muscular lipid storage is not detrimental for insulin sensitivity, but rather is protective. What the exact mechanisms are behind this is potentially interesting for the pharmaceutical industry. However, more mechanistically studies are needed to find which pathway needs to be targeted to store lipids in a non-detrimental way for insulin sensitivity.

Although (re)introducing physical activity in a sedentary lifestyle is not easy, the results from this thesis underpin the notion that general practitioners and physiotherapists should stimulate T2DM patients and people with overweight/obesity to become more physically active or providing exercise programs for this patient group. However, a more detailed understanding is still needed for the group of T2DM patients who do not improve their insulin sensitivity upon exercise. 


\section{Activities and products}

The research executed in this thesis is performed at the department of Human Biology and Human Movement Sciences at the Maastricht University in the group of Prof. dr. M.K.C. Hesselink and Prof. dr. P. Schrauwen. This research group is worldwide recognized in performing translational studies in the field of T2DM and is mainly focused on elucidating the underlying mechanisms of insulin resistance of mainly skeletal muscle. In this thesis we took the advantage to analyze muscle biopsies taken in human studies in more detail regarding lipid droplet characteristics with confocal fluorescence microscopy. These microscopic studies were performed in cooperation with the department of Genetics \& Cell Biology, division Molecular Biology of the Maastricht University. In addition, the measurements for chemical lipid droplet composition were performed in collaboration with the department of Molecular Spectroscopy of the Max Planck Institute in Mainz.

Results presented in this thesis have been implemented in original scientific articles. These articles are published in or submitted to well-recognized peer-reviewed journals. These articles are available online and can be accessed by scientist all around the world. In addition, knowledge obtained from these studies is also communicated to the scientific community via presentations and posters at national and international conferences.

\section{Innovation}

A classical way to study lipid storage in skeletal muscle is by biochemically extract triglycerides from homogenates. These lipid extractions only give insights in concentration, but not in location (where in the muscle fiber or which fiber type), lipid droplet size and number. The use of microscopy can image these lipid droplet characteristics. Electron microscopy is a widely used technique to study lipid droplet size, number and location. However, this type of microscopy does not give insight in fiber type differences. Fluorescence microscopy can measure fiber type specific and quite some studies have used this type of microscopy to study lipid droplet characteristics per fiber type. In this thesis high-resolution confocal scanning laser microscopy was used to study lipid droplet morphology, decoration and location. The field of high-resolution microscopy is developing quickly. Super-resolution microscopy techniques like STimulated Emission Depletion (STED), a technique awarded the 2014 Nobel Prize in Chemistry, can theoretically reach spatial resolutions down to the $30 \mathrm{~nm}$ level. STED microscopy is nowadays accessible for biomedical scientist and is well on its way to become the new golden standard for colocalization/interaction studies. We have set-up STED imaging to study the putative presence of PLIN5 in the mitochondria. The resolution was high enough to distinguish the inner and outer mitochondrial membrane. This gave us the opportunity to 
examine if PLIN5 is present on the inner or outer mitochondrial membrane, or is in close proximity of mitochondria. Besides studying lipid droplet morphology and location we applied Coherent Antistokes Raman Spectroscopy (CARS) to examine the chemical lipid droplet composition. In contrast to the classical way to study lipid composition, no lipid extractions are needed for CARS and lipids can be studied without the need of labels in situ. This technique will give the chemical composition (level of saturation and chain length) of lipid droplets only and can make a distinction between type I and type II fibers when combined with fluorescence microscopy.

We combined the data of lipid droplet characteristics at the level of muscle fibers with whole body physiological data obtained in our lab. Lipid droplet characteristics were studied in relation to insulin sensitivity (measured by a hyperinsulinemic euglycemic clamp) and maximal oxidative capacity. Besides cross-sectional studies we studied changes in lipid droplet characteristics in healthy lean subjects upon interventions that challenge the muscle fibers to handle a high flux of fatty acids. Although these type of studies promote our understanding of how fatty-acid flux is handled by the muscle and sheds novel light on the importance of lipid droplet dynamics in maintaining insulin sensitivity these human studies do not provide causality. Nevertheless, our partly associative studies are important key for the translation of pre-clinical research towards the human situation.

\section{Planning and realization}

In this thesis, we observed that PLIN5 coated lipid droplets can be protective against the development of insulin sensitivity. However, this is not always the case; South Asians still develop insulin resistance upon a high-fat high-calorie diet despite an increase in PLIN5 protein content. In addition, number of PLIN5 coated lipid droplets and PLIN5 protein content are only associated with levels of insulin sensitivity when maximal oxidative capacity is taken into account. More research is warranted to study role of skeletal muscle lipid droplet dynamics in relation to insulin sensitivity. I will continue to perform human studies whereby imaging techniques, like fluorescence microscopy and mass spectrometry imaging, are combined with whole body physiological data to study the role of lipid droplet and mitochondrial dynamics in skeletal muscle insulin resistance. 


\section{RISK ANALYSIS AND TECHNOLOGY ASSESSMENT}

NanoNextNL financially supported the work presented in this thesis. NanoNextNL is a consortium consisting of more than 100 firms, universities, knowledge institutes and university medical centers. This consortium financed research involving micro and nanotechnology (www.nanonextnl.nl). Important aspects that are easily overlooked for development of micro and nanotechnology are the assessment of the technology's safety, its acceptance by the society and what the possible impact of the potential newly developed technology is. These assessments are summarized by the term Risk Analysis and Technology Assessment (RATA). This thesis focused on intramyocellular lipid droplets in relation to insulin sensitivity. This does not directly relate to nanotechnology, however, all research performed has a certain impact on society which is also a part of RATA. This appendix will focus on two questions in relation to RATA: first, we assess the additional knowledge that would be required to apply the findings in this thesis in practical treatment and prevention programs. Second, we assess the impact of variations among ethnicity in this type of advice.

In chapters 4, 5 and 6 we showed that a high level of intramyocellular lipids (IMCL) per se does not necessarily lead to insulin resistance of the skeletal muscle. Endurance trained athletes have similar, or even higher, levels of IMCL as type 2 diabetic (T2DM) subjects. In the late 90 s it was shown for the first time in humans that levels of $I M C L$ were negatively associated with insulin sensitivity $[1,2]$, meaning that the more IMCL you have the less insulin sensitive you are. However, this association is absent in endurance trained athletes [3]. Furthermore, we have shown that lean healthy subjects who are able to store the highest levels of IMCL during 60 hours of fasting become the least insulin resistance. Concluded from these chapters is that high levels of IMCL content does not necessarily need to lead to the development of insulin resistance. The way IMCL is stored (as in more and smaller lipid droplets (LDs), in type I fibers and the presence of perilipin 5) is putatively a more important determinant of insulin sensitivity. So, the relationship between IMCL content and insulin sensitivity is not straightforward. Understanding the fundamental differences in IMCL storage in type 2 diabetes patients and athletes cannot be directly applied in treatment and prevention programs.

\section{Exercise interventions, insulin sensitivity and IMCL content}

The fundamental results reported in this thesis about IMCL content and the way myocellular lipid storage affects insulin sensitivity needs some further examination before applying these findings into a treatment and prevention program. Literature has already shown that training programs can change how IMCL is stored without affecting total IMCL content. It is not only shown that training 
reduces the size of LDs [4], but that at specific cellular locations IMCL content is decreased upon training [5]. These changes were in the same directions as how athletes stored their IMCL examined in this thesis; smaller LDs and less IMCL content in the subsarcolemmal fraction (chapter 6). However, not all type 2 diabetes patients respond to training regarding glucose homeostasis or insulin sensitivity, this varies between 7 to $63 \%$ among studies [6]. In chapter 4 a difference in response to $\mathrm{IMCL}$ content upon prolonged fasting is observed. Interestingly, subjects who increased $I M C L$ content the most were protected against fasting induced insulin resistance. So, putatively the diversity in training response on insulin sensitivity might be due to a different adaptation of IMCL storage upon training. A potentially underlying mechanism for a differential adaptive response might be the functioning of the LDs. Lipid droplets are nowadays recognized as dynamic organelles involved in releasing and storing fatty acids and not as simple storage sites for IMCL. From cardiac muscle it is known that static LDs, i.e. LDs which are unable to release fatty acids for oxidation, contribute to lethal cardiomyopathy [7]. The increase in IMCL content upon prolonged fasting was entirely due to an increased in PLIN5 coated IMCL, suggesting more dynamic LDs since PLIN5 is involved in regulating the release and storage of fatty acids from LDs [8]. At the moment it is reported that storage of $I M C L$ in smaller LDs, in the intermyofibrillar cellular region and coating with PLIN5 is associated with high levels of insulin sensitivity. In addition, training adapts IMCL storage to the way endurance trained athletes store their IMCL content $[4,5]$. However, a direct causal link for IMCL content with insulin sensitivity needs to be established. Furthermore, it is unknown if training induces changes in IMCL storage in non-responding type 2 diabetes patients on insulin sensitivity and if these non-responders respond on a diet.

Not only responsiveness to training program with respect to insulin sensitivity is of importance, but it is rather likely that also the training modality, like low/high intensity, endurance/resistance/interval training and duration affects the routes involved and hence the outcome. It is non-conclusive what the best training modality is for improving insulin sensitivity [9]. In addition, it is unknown if high intensity interval training is able to change IMCL storage in such a way as that of endurance trained athletes or upon endurance training at $60-70 \%$ of maximal oxidative capacity. Is just walking sufficient, do these patients need to do more intense exercise or is a combination between endurance and resistance training preferred? Taken together with the nonresponders, this suggests that personalized prevention and intervention programs under supervision are more suitable for combating type 2 diabetes. To come to a personalized intervention/prevention program, non-responders and/or how patients would respond to a certain program should be recognized quickly. More knowledge should become available why non-responders do not respond to exercise on certain parameters, like insulin sensitivity, and if they respond to another type of 
intervention. Is there a certain protein not working properly resulting in dysfunction of organelles like mitochondria and/or lipid droplets, or do they have a certain mutation in some gene-sets important for a training response?

\section{Differences between ethnicities}

Results from chapter 2 suggest a second consideration that should be taken into account for prevention and intervention programs: ethnicity. In almost all chapters in this thesis and in most studies, the relationship between IMCL content and insulin sensitivity is investigated in subjects from Caucasian descent. However, it is doubtful if the results found in Caucasian subjects are directly translatable towards the whole population consisting of multiple ethnicities. This is an important question to consider, because Western society becomes more and more multicultural. In sedentary Caucasian subjects negative associations are found between levels of IMCL content and insulin sensitivity $[2,10]$, while these associations are not found in South Asians [11]. This might suggest that in contrast to a Caucasian population the level of IMCL content is unrelated to insulin sensitivity and therefore less important in the development of type 2 diabetes for South Asians. In chapter 2 we further investigated the relationship between IMCL and insulin sensitivity in South Asians and compared this with Caucasians. Furthermore, we examined if these two populations have a similar response on a high fat diet for 5 days. These comparisons between Caucasians and South Asians regarding the difference in the relationship between $I M C L$ and insulin sensitivity and responses on diet and/or exercise interventions on insulin sensitivity and IMCL storage are important for developing future interventions for preventing the development of T2DM. Importantly, more ethnicities should be included in these types of studies. Is the response of diets (high-fat or low-fat) and exercise interventions on metabolic parameters (insulin sensitivity, mitochondrial function, fat oxidative capacity etc.) similar among ethnicities or is a certain intervention or combination of interventions better suitable for a certain ethnicity? All this information taken together with studies investigating the involvement of genes or slightly modified genes in the progression of insulin resistant could contribute to the development of personalized treatment programs.

\section{Conclusion}

The results from this thesis gives us a better understanding how IMCL content is related to the development of insulin resistance. The relationship between IMCL content and insulin sensitivity is 
not straightforward. To further implement the results from this thesis in treatment/prevention programs more studies are needed regarding the training effect in T2DM patients, people at risk of developing T2DM and other ethnicities. In addition, little is known why non-responders do not improve insulin sensitivity upon a training intervention and if these patients do improve their insulin sensitivity upon another type of intervention. Understanding the response (or non-response) on exercise interventions lead to better prevention and/or treatment programs for T2DM.

\section{References}

1. Pan, D.A., et al., Skeletal muscle triglyceride levels are inversely related to insulin action. Diabetes, 1997. 46(6): p. 983-8.

2. Krssak, M., et al., Intramyocellular lipid concentrations are correlated with insulin sensitivity in humans: a $1 H$ NMR spectroscopy study. Diabetologia, 1999. 42(1): p. 113-6.

3. Goodpaster, B.H., et al., Skeletal muscle lipid content and insulin resistance: evidence for a paradox in endurance-trained athletes. J Clin Endocrinol Metab, 2001. 86(12): p. 5755-61.

4. He, J., B.H. Goodpaster, and D.E. Kelley, Effects of weight loss and physical activity on muscle lipid content and droplet size. Obes Res, 2004. 12(5): p. 761-9.

5. Nielsen, J., et al., Increased subsarcolemmal lipids in type 2 diabetes: effect of training on localization of lipids, mitochondria, and glycogen in sedentary human skeletal muscle. Am J Physiol Endocrinol Metab, 2010. 298(3): p. E706-13.

6. Bohm, A., et al., Exercise and diabetes: relevance and causes for response variability. Endocrine, 2016. 51(3): p. 390-401.

7. Haemmerle, G., et al., ATGL-mediated fat catabolism regulates cardiac mitochondrial function via PPAR-alpha and PGC-1. Nat Med, 2011. 17(9): p. 1076-85.

8. Sanders, M.A., et al., Endogenous and Synthetic ABHD5 Ligands Regulate ABHD5-Perilipin Interactions and Lipolysis in Fat and Muscle. Cell Metab, 2015. 22(5): p. 851-60.

9. Stephens, N.A. and L.M. Sparks, Resistance to the beneficial effects of exercise in type 2 diabetes: are some individuals programmed to fail? J Clin Endocrinol Metab, 2015. 100(1): p. 43-52.

10. Goodpaster, B.H., et al., Intramuscular lipid content is increased in obesity and decreased by weight loss. Metabolism, 2000. 49(4): p. 467-72.

11. Forouhi, N.G., et al., Relation of triglyceride stores in skeletal muscle cells to central obesity and insulin sensitivity in European and South Asian men. Diabetologia, 1999. 42(8): p. 932-5. 


\section{DANKWOORD}

Het is dan eindelijk zover; mijn boekjes is af! Het promotietraject was een leerzame ervaring. Niet alleen wetenschappelijk, maar ook op persoonlijk vlak. Zonder de hulp van anderen was dit boekje niet tot stand gekomen.

Allereest wil ik graag mijn promotieteam, Matthijs, Patrick en Marc, bedanken. Tijdens het lopen van mijn stage binnen de SHOCk-groep werd me duidelijk dat ik graag verder wilde in het onderzoek van onderliggende mechanisme van insulineresistentie. Matthijs, bedankt dat je me de kans hebt gegeven om verder te gaan in dit onderzoek. Ik heb samenwerking als prettig ervaren en veel van je geleerd. De trip naar Snowmass zal ik niet zo gauw vergeten. Na onder andere een avontuurlijk taxiritje en een nachtje 'kamperen' op het vliegveld van Denver wisten we er eindelijk te komen. Dat was deze reis meer dan de moeite waard, want ik heb veel van het congres kunnen opsteken en we hebben er verdere stappen in het vetdruppelonderzoek mee kunnen zetten. Patrick, je was in mijn project wat meer op de achtergrond aanwezig en waar je kon was je aanwezig bij onze meetings. Vaak zorgde jouw aanwezigheid tijdens deze meetings voor een weer frissere blik op de data en hoe verder te gaan met project. Marc, onze samenwerking begon wat later in mijn promotietraject en is heel waardevol gebleken. Dankzij deze samenwerking hebben we flinke stappen weten te zetten op het in beeld brengen van vetdruppels en de coating van deze druppels en heb ik veel geleerd wat betreft fluorescentiemicroscopie.

Thanks to the members of the assessment committee Ronald Mensink, Bret Goodpaster, Ron Heeren, Cedric Moro and Frans Ramaekers to take time to read and review my thesis. Bret and Cedric, thanks for coming over for my defense.

Femke en Carlijn, fijn dat jullie mijn paranimfen willen zijn! Femke, we kennen elkaar al een tijdje. In de $3^{\mathrm{e}}$ klas van de middelbare school kwamen we bij elkaar in de klas. Samen hebben we onze allereerste stapje gezet in het onderzoek tijdens ons profielwerkstuk. Daarna zijn we ieder ons weg gegaan en beide op een heel ander onderzoeksgebied terechtgekomen. Ondanks dat we niet meer zo dicht bij elkaar wonen hebben we nog steeds contact en als we elkaar weer zien is het als vanouds en doen we vaak mee aan een loopje. Hopelijk kunnen we dit nog een tijdje doen! Succes met de laatste loodjes van je promotieonderzoek! Carlijn, we kennen elkaar wat korter, maar een klik was snel gevonden. Naast dezelfde wetenschappelijke interesse, hebben we allebei een passie voor het hardlopen. Al zal dat volgens sommige van JustGoo meer kletsen tijdens het hardlopen zijn... Jij ook succes met je promotieonderzoek en het maken van mooie verre reizen! 
Mijn (ex)collega's van SHOCk, bedankt voor de gezelligheid en de samenwerkingen. Sabine, een paar jaar geleden kwam je erbij en was ik niet meer de enige PhD student die bezig was met in het donker vetdruppeltjes te tellen en meten. Een prettige samenwerking die we nog even kunnen voortzetten. Nynke, je bent pas bij het vetdruppelteam gekomen. Succes gewenst met je promotietraject. Joris en Bram, bedankt voor het beschikbaar stellen van de spierbiopten die zijn afgenomen in jullie studies. Lauren, thanks for your enthusiasm for science during my internship. It made me decide to continue to do research in this field. Esther en Johanna, bedankt voor jullie hulp en ondersteuning op het lab. Lotte, Carlijn, Vera, Kay and Evelyn, thanks for the gezellige lunch/after work runs.

Gert en Helma ik wil jullie bedanken voor de samenwerkingen met het opzetten van kleuringen en de metingen op de confocale en STED-microscoop. Jullie enthousiasme bij het zien van details of resolutieverbeteringen tijdens het imagen is aanstekelijk.

Sapun and Nils, thanks for giving me the opportunity to measure lipid composition with CARS microscopy. A different type of microscopy that I was used, but an experience I never forget.

Pap, mam en Marije bedankt voor jullie ondersteuning tijdens mijn studie en het verdere vervolg. Het is niet altijd even makkelijk voor jullie geweest dat ik naar het zuiden van Nederland ben verhuisd. Pap en mam, de kinderen zijn het huis uit en jullie hebben het 'rijk' weer voor jullie zelf, behalve dan tijdens de kerstvakanties. Altijd fijn om even een week er tussenuit te zijn en de batterij op te laden. Pap, sterkte met de laatste loodjes! Het aftellen naar je pensioen is begonnen. lets waar je al een tijdje naar uitkijkt. Mam, voor jou is het nog wat langer, maar ook dat komt eraan. Mogen jullie samen gelukkig oud worden! Marije, na verschillende studies, vrijwilligerswerk in Ghana en baantjes weet je nu wat je leuk vindt om te doen. Je bent begonnen aan je HBO toegepaste wiskunde en gaat ontzettend goed. Nu kan ik jou een nerd kan noemen ;-). Ik ben trots op je! Nu ik zelf niet meer badminton, kunnen we niet meer samen dubbelen op toernooien (Heemskerk zal ik nooit vergeten; een tweede plek met een gouden randje), maar het samen lopen van een lokale Kerstloop afgelopen Kerst voelde wel weer een beetje aan als het samen dubbelen. Nu kan ik ook eindelijk af en toe bij jou competitiewedstrijden kijken en zie ik het fanatisme terug dat ik een aantal jaren terug ook had. Jullie zijn verdiend gepromoveerd naar de derde divisie. Succes met je studie en het behouden in de derde divisie en veel geluk met Derbert toegewenst! 


\section{PUBLISHED MANUSCRIPTS}

Gemmink A, Bakker LEH, Guigas B, Kornips E, Schaart G, Meinders EA, Jazet IM, Hesselink MKC. Lipid droplet dynamics and insulin sensitivity upon a 5-day high-fat diet in Caucasians and South Asians. Sci. Rep. 7, 42393; doi: 10.1038/srep42393 (2017). Impact factor: 5.228

Sparks LM, Gemmink A, Phielix E, Bosma M, Schaart G, Moonen-Kornips E, Jörgensen JA, Nascimento EB, Hesselink MK, Schrauwen P, Hoeks J. ANT1-mediated fatty acid-induced uncoupling as a target for improving myocellular insulin sensitivity. Diabetologia, 2016. 59(5):1030-9. Impact factor: 6.206

Gemmink A*, Bosma M*, Kuijpers HJ, Hoeks J, Schaart G, van Zandvoort MA, Schrauwen P, Hesselink MK. Decoration of intramyocellular lipid droplets with PLIN5 modulates fasting-induced insulin resistance and lipotoxicity in humans. Diabetologia, 2016. 59(5):1040-8. Impact factor: 6.206

*These authors contributed equally

van Ewijk PA, Paglialunga S, Kooi ME, Nunes PM, Gemmink A, Slenter J, Kornips E, Jörgensen JA, Hoeks J, Wildberger JE, Hesselink MK, Glatz JF, Heerschap A, Kersten S, Schrauwen P, SchrauwenHinderling VB. Effects of high-fat feeding on ectopic fat storage and postprandial lipid metabolism in mouse offspring. Obesity, 2015. 23(11):2242-50. Impact factor: 3.614

Hanssen MJ, Wierts R, Hoeks J, Gemmink A, Brans B, Mottaghy FM, Schrauwen P, van Marken Lichtenbelt WD. Glucose uptake in human brown adipose tissue is impaired upon fasting-induced insulin resistance. Diabetologia, 2015. 58(3):586-95. Impact factor: 6.206

Gemmink A, Hesselink M. Intramyocellular lipid storage and insulin resistance [internet]. 2015 Feb 11. Diabepedia 3105512811 rev. No. 7. Available from: http://dx.doi.org/10.14496/dia.3105512811.7

Billecke N, Rago G, Bosma N, Eijkel G, Gemmink A, Leproux P, Huss G, Schrauwen P, Hesselink MK, Bonn M, Parekh SH. Chemical imaging of lipid droplets in muscle tissues using hyperspectral coherent Raman microscopy. Histochem Cell Biol, 2014. 141(3):263-73. Impact factor: 2.78

de Ruiter CJ, Hutter V, Icke C, Groen B, Gemmink A, Hilde S, de Haan A. The effects of imaginary training on fast isometric knee extensor torque development. J Sports Sci, 2012. 30(2):166-74. Impact factor: 2.142 


\section{MANUSCRIPTS IN PREPARATION}

Gemmink A*, Daemen S*, Brouwers B, Huntjens PR, Schaart G, Hoeks J, Schrauwen P, Hesselink MKC. Dissociation of intramyocellular lipid storage and insulin resistance in trained athletes and patients with type 2 diabetes; involvement of the lipid droplet coat protein Perilipin 5. In preparation *These authors contributed equally

Gemmink A*, Daemen S*, Billecke N, Rieger K, Brouwers B, Schaart G, Fleissner F, Hoeks J, Schrauwen $\mathrm{P}$, Parekh SH, Hesselink MKC. Different intramyocellular lipid storage pattern and level of lipid droplet saturation in athletes and type 2 diabetes patients. In preparation

*These authors contributed equally 


\section{CURRICULUM VITAE}

Anne Gemmink was born on January $8^{\text {th }} 1989$ in Naarden, the Netherlands. In 2007 she finished preuniversity secondary education at het Goois Lyceum in Bussum, the Netherlands. Afterwards, she started with the bachelor Human Movement Sciences at the Free University in Amsterdam. During her bachelor internship she investigated whether training of fast isometric knee extensor torque development was knee angle specific under supervision of dr. Jo de Ruiter. This internship resulted in a publication in the Journal of Sport Sciences. She graduated in 2010 within the specialization Movement Systems.

Subsequently, she moved to the Maastricht University to continue with the Master Physical Activity and Health within the specialization of Biology of Human Performance and Health. During her master internship she investigated the role of ANT1 in mitochondrial uncoupling of skeletal muscle under supervision of $d r$. Joris Hoeks and dr. Lauren Sparks at the department of Human Biology at the same university. This internship resulted in a publication in Diabetologia. In 2011 she graduated and received the Top 3\% award for belonging to the top 3\% of best students of the Faculty of Health Medicine and Life Sciences.

In September 2011 she started with her PhD at the department of Human Movement Sciences at the Maastricht University under supervision of Prof. dr. Matthijs Hesselink, Prof. dr. Patrick Schrauwen and Prof. dr. Marc van Zandvoort. After pioneering studies on the role of FSP27 in skeletal muscle, she switched topic and focused on skeletal muscle lipid droplet morphology, location, composition and coating with PLIN5 in the (patho)physiology of insulin sensitivity. During her studies she was involved in setting up (3D) confocal and STED imaging in skeletal muscle tissue for further studying lipid droplet morphology and protein decoration.

Anne Gemmink is currently working as a postdoctoral researcher at the department of Human Biology and Movement Sciences at the Maastricht University. She is involved in applying and optimizing mass spectrometry imaging techniques on skeletal muscle to study lipid composition in relation to insulin sensitivity in a location specific manner. In addition, she will continue working on STED imaging of lipid droplet coating proteins and their relation with insulin sensitivity. 